Torção Analítica e extensões para o Teorema de Cheeger Müller

\author{
Luiz Roberto Hartmann Junior
}



SERVIÇO DE PÓS-GRADUAÇÃO DO ICMC-USP

Data de Depósito: 23 de novembro de 2009

Assinatura:

\title{
Torção Analítica e extensões para o Teorema de Cheeger Müller
}

\author{
Luiz Roberto Hartmann Junion 1
}

Orientador: Prof. Dr. Mauro Flavio Spreafico

Tese apresentada ao Instituto de Ciências Matemáticas e de Computação - ICMC/USP, como parte dos requisitos para obtenção do título de Doutor em Ciências Matemática.

\section{USP - São Carlos}

Novembro/2009

\footnotetext{
${ }^{1} \mathrm{O}$ autor teve apoio financeiro da CAPES de 03/07 até 11/07 e da FAPESP, processo 2007/05452 - 6, de $12 / 07$ até $12 / 09$
} 

Aos meus avós, Heinz

(In memoriam) $e$ Lydia, Anésio e Maria (In memoriam). 



\section{Agradecimentos}

Em primeiro lugar, agradeço a Deus por ter guiado todos os meus passos até aqui.

Agradeço a meus pais, Luiz e Ana, por todo apoio, educação e incentivo. Aos meus irmãos, Hannah e Marcelo; como diria nossa avó Lydia "Vocês são deferentes, mas são 11". Ao meu Tio Paulo, minha Tia Ana e minha avó Lydia por sempre terem me ajudado.

À minha esposa Carla por seu companheirismo, compreensão e paciência. Você sabe da sua importância. Ao Branco e a Maria por terem me recebido em sua família como se eu fosse mais um de seus filhos.

Ao Prof. Mauro Spreafico, por sua paciência e disposição em sanar todas as minhas dúvidas nestes anos de mestrado e doutorado, e ainda, por me apresentar o caminho da pesquisa. Ao Prof. Dide por ter me ajudado sempre que precisei. Aos grandes professores Castelo, Ronaldo, Robson, José Roberto, Biroca, Gabriel, Mônica, Marcelo Messias, Messias Meneguette, Adalberto, Cidinha, Eduardo, Daniel Smania e Carlos Biasi, cujos cursos fantásticos tive a honra de assistir em diferentes etapas da minha formação.

Aos colegas de pós-graduação Ubarana, Edinho, John, Pimenta, Claudinei, Mário, Marcão, Kleyber e Thais, com os quais mantive algumas discussões matemáticas interessantíssimas. Em especial agradeço ao Prof. Marcio Colombo Fenille, pela leitura e sugestões dadas a este texto, e ao Prof. Thiago de Melo, pelas figuras que se encontram nesta tese e a ajuda com o LATEX.

A todos os funcionários do ICMC sempre dispostos a ajudar no que for preciso.

Por fim a CAPES e a FAPESP pelo apoio financeiro. 


\section{Resumo}

Estudamos a Torção Analítica para variedades com bordo e ainda com singularidades do tipo cônico, mais especificamente, para um cone métrico limitado, com o propósito de investigar a extensão natural do Teorema de Cheeger Müller para tais espaços. Começamos determinando a Torção Analítica do disco e de variedades com o bordo totalmente geodésico, por meio de ferramentas geométricas desenvolvidas por J. Brüning e X. Ma. Posteriormente, usando ferramentas analíticas desenvolvidas por M. Spreafico, determinamos a Torção Analítica do cone sobre uma esfera de dimensão ímpar e provamos um teorema do tipo Cheeger Müller para este espaço. Mais ainda, provamos que o resultado de J. Brünning e X. Ma estende para o cone sobre uma esfera de dimensão ímpar. 


\section{Abstract}

We study the Analytic Torsion of manifolds with boundary and also with conical singularities, more specifically, for a finite metric cone, with the purpose of investigating the natural extension of the Cheeger Müller theorem for such spaces. We start by computing the Analytic Torsion of an any dimensional disc and of a manifold with totally geodesic boundary, by using geometric tools developed by J. Brünng and X. Ma. Then, by using analytic tools developed by M. Spreafico, we determine the Analytic Torsion of a cone over an odd dimensional sphere and we prove a theorem of Cheeger Müller type for this space. Moreover, we prove that the result of J. Brüning and X. Ma extends to the cone over an odd dimensional sphere. 


\section{Sumário}

Agradecimentos vii

Resumo ix

Abstract $\quad$ xi

$\begin{array}{ll}\text { Introdução } & 1\end{array}$

1 Preliminares $\quad$ 5

1.1 Tensores e formas diferenciais . . . . . . . . . . . . . . . . . 5

1.2 Geometria Riemanniana . . . . . . . . . . . . . . . . . . . . 9 9

1.3 Teoria de Hodge . . . . . . . . . . . . . . . . . . . . . . 12

1.4 Variedades com singularidades do tipo cônico . . . . . . . . . . . . . . 14

1.5 Ferramentas analíticas . . . . . . . . . . . . . . . . . . . 17

2 A Torcão de Reidemeister e a Torção Analítica 23

2.1 Torcão de Reidemeister . . . . . . . . . . . . . . . . . . . . . . . . 23

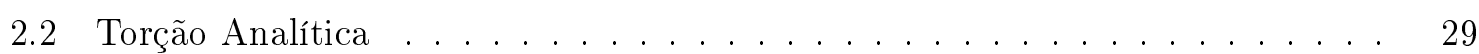

2.3 Motivacão para a definicão da Torção Analítica . . . . . . . . . . . . . . . . . 32

2.4 Teoremas de comparacão . . . . . . . . . . . . . . . . . . . . . . 33

3 Torcão Analítica de variedades com bordo totalmente geodésico $\quad 37$

3.1 Variedades com o bordo totalmente geodésico . . . . . . . . . . . . . . 37

3.2 Torcão Analítica das meia-esferas . . . . . . . . . . . . . . . . . . . . 39

3.3 Torção Analítica da calota esférica . . . . . . . . . . . . . . . . . . . 41

\begin{tabular}{|ll|l}
4 & $\mathrm{O}$ disco $m$-dimensional & 47
\end{tabular}

4.1 Torcão de Reidemeister do cone sobre uma esfera . . . . . . . . . . . . . . . 47

4.2 A Torcão Analítica do disco m-dimensional . . . . . . . . . . . . . . . . 50

4.3 Torcão Analítica de $C_{\alpha} S_{1}^{1}$ e $C_{\alpha} S_{1}^{2} \quad \ldots \ldots \ldots$. . . . . . . . . . . 57

4.3 .1 Espectro do Laplaciano sobre as formas . . . . . . . . . . . . . . 58

4.3 .2 Torção Analítica do cone sobre o círculo . . . . . . . . . . . . . . . . 69 
4.3.3 Torção Analítica do cone sobre uma esfera . . . . . . . . . . . . . . 73

5 A conjectura para esferas de dimensão ímpar $\quad 81$

5.1 Dimensão Baixa . . . . . . . . . . . . . . . . . . . . . 81

5.2 Motivação para a generalização no caso ímpar e a conjectura . . . . . . . . . . 97

6 Torcão Analítica do cone sobre uma variedade de dimensão ímpar 103

6.1 Cone sobre uma variedade fechada conexa de dimensão ímpar . . . . . . . . . 103

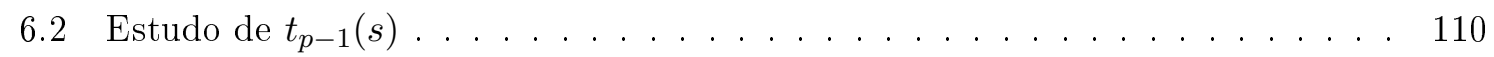

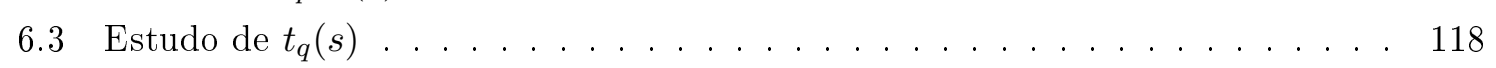

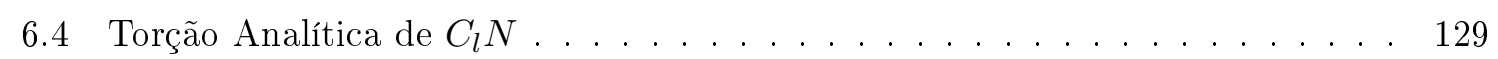

6.5 Prova da conjectura . . . . . . . . . . . . . . . . . . 132

\begin{tabular}{|l|l|l|}
\hline Referências Bibliográficas & 145
\end{tabular}

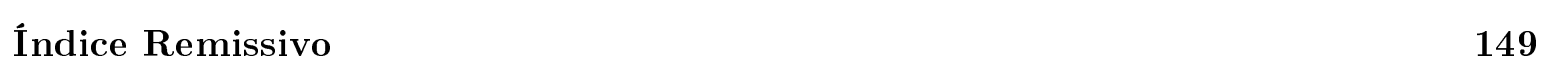




\section{Introdução}

A Torção de Reidemeister foi definida primeiramente para complexos simpliciais finitos por K. Reidemeister [38] e W. Franz [19], com o objetivo de classificar os espaços lenticulares. Estes, por sua vez, foram os primeiros exemplos de variedades que possuem o mesmo tipo de homotopia sem serem homeomorfos. Tais espaços foram completamente classificados por meio da Torção de Reidemeister. Desta forma a Torção de Reidemeister foi o primeiro invariante de uma variedade que não é invariante por tipo de homotopia. Com isto, pode-se entender que a Torção de Reidemeister consegue identificar a estrutura de interações entre o grupo fundamental e a estrutura simplicial. Citando Milnor [30]: "Seja $X$ um complexo simplicial finito com grupo fundamental $\pi_{1}(X)$ cíclico de ordem $n$. Identificamos $\pi_{1}(X)$ com o grupo das transformações de recobrimento do recobrimento universal simplicial $\tilde{X}$. Se $\pi_{1}(X)$ age trivialmente na homologia racional $H_{*}(\tilde{X} ; \mathbb{Q})$, então a torção de $X$ é definida como uma certa coleção de elementos do corpo algébrico de números $\mathbb{Q}\left[\mathrm{e}^{\frac{2 \pi i}{n}}\right]$. Esta torção é uma espécie de determinante que descreve a maneira como os simplexos de $\tilde{X}$ são colados entre si pela ação do grupo fundamental".

Em [51] J.H.C. Whitehead criou um invariante ainda mais refinado. Ele definiu a torção de uma equivalência de homotopia entre complexos celulares finitos, generalizando a Torção de Reidemeister. Este invariante, chamado de Torção de Whitehead, é um invariante mais delicado, definido não como um número algébrico, mas como um elemento de um certo grupo abeliano. No mesmo ano, G. de Rham [16] estendeu o conceito da Torção de Reidemeister

para uma variedade suave. Na década de 70, D. B. Ray e I. M. Singer [36], com o teorema do índice de Atiyah-Singer em mente, procuraram uma descrição analítica para a Torção de Reidemeister seguindo uma sugestão de A. Shapiro. A. Shapiro sugeriu que deveria existir uma fórmula para a torção em termos do Laplaciano que age nas formas diferenciais. Como a Torção de Reidemeister depende da base da homologia fixada (ver seção 2.1), Ray e Singer [36] fixaram a base da homologia escolhendo uma base ortonormal das formas harmônicas. Mais precisamente, sejam $W$ uma $m$-variedade Riemanniana, compacta e orientada, $K$ o complexo celular de uma triangulação suave de $W, \rho$ uma representação do grupo fundamental por matrizes ortogonais. Considere uma base ortonormal para as formas harmônicas de $W$ com valores no fibrado vetorial $E_{\rho}$ associado à representação $\rho$. Pela a dualidade de LefschetzPoincaré e os isomorfismos de de Rham e Hodge, a base ortonormal das formas harmônicas 
é levada em uma base da homologia de $W$ e esta é a base da homologia fixada na Torção de Reidemeister por Ray e Singer. Trabalhamos com a Torção de Reidemeister sempre neste contexto.

Ainda em [36], Ray e Singer definem a Torção Analítica como um invariante do complexo de de Rham com coeficientes no fibrado vetorial $E_{\rho}$ e provam várias propriedades para a Torção Analítica, as quais Torção de Reidemeister também satisfaz. Eles ainda apresentam uma motivação para esta definição (ver seção 2.3). Com isso, Ray e Singer conjecturaram que a Torção de Reidemeister e a Torção Analítica eram iguais, ao menos quando $W$ é fechada. No final da década de 70, J. Cheeger [6] e W. Müller [32] provaram, de maneira independente, que a Torção Analítica e a Torção de Reidemeister são iguais quando $W$ é uma variedade Riemanniana fechada. Ambas as provas são complexas e trabalhosas. A prova de W. Müller envolve a teoria combinatória de Hodge com a teoria de aproximação, enquanto a prova de J. Cheeger envolve teoria de cirurgia. Esta igualdade entre as duas torções ficou conhecida como o célebre Teorema de Cheeger Müller. Desde então a Torção Analítica passou a ter uma importância independente tanto em Geometria e Topologia Diferencial quanto na Análise Global. Foram introduzidas e estudadas várias extensões. Lembramos aqui os trabalhos de J. Lott e M. Rothemberg [26], W. Lück [27] e J. M. Bismut e W. Zhang [3], onde as versões equivariantes e $L^{2}$ da Torção Analítica foram introduzidas e estudadas. Apesar de existir uma contribuição grande no sentido qualitativo da Torção Analítica, resultados quantitativos não são muitos. Podemos citar, por exemplo, [15], [37] e [50]. Nessa linha determinamos a Torção Analítica das calotas esféricas e do cone sobre $S^{1}, S^{2}$ e $S^{3}$. Com estes três últimos temos também a Torção Analítica dos discos de dimensão 2, 3 e 4.

Uma questão natural no estudo do Teorema de Cheeger Müller é a extensão deste resultado para variedades com bordo. Em [6], J. Cheeger afirma, mas não prova, que a Torção Analítica de uma variedade Riemanniana $W$, compacta e com bordo, é a Torção de Reidemeister acrescida de um termo $C(\partial W)$ que depende do bordo. Este termo $C(\partial W)$ foi determinado por W. Lück em [27], no caso de variedades em que a métrica tem estrutura produto próxima ao bordo. No capítulo 3 apresentamos uma extensão deste resultado para variedades com o bordo totalmente geodésico. Nesta situação, $C(\partial W)$ é proporcional a característica de Euler do bordo de $W$. Recentemente, sem assumir que a métrica tem a estrutura produto próxima ao bordo, X. Dai e H. Fang [13] e J. Brüning e X. Ma [4] resolveram o problema apresentando um teorema do tipo Cheeger Müller. Ambas as fórmulas são complicadas, apesar de serem baseadas em invariantes locais construídos a partir da métrica (2-forma curvatura), são definidas utilizando a integral de Berezin, teoria de Chern-Simon e resultados recentes de J. M. Bismut e W. Zhang [3]. Nos dois trabalhos, surge um novo termo, $A(\partial W)$, chamado de termo anômalo do bordo (ver seção 2.4). O que chama a atenção é que o termo $A(\partial W)$ de [13] difere do termo $A(\partial W)$ de [4]. Com isto, torna-se interessante a determinação do termo correto. Este problema é resolvido nos capítulos 4 e 5, onde por cálculos diretos determinamos a Tor- 
ção Analítica dos discos de dimensão 3 e 4 e comparamos com os resultados obtidos através das fórmulas de [13] e [4]. Os resultados obtidos concordam com a fórmula de [4], servindo como um contra-exemplo para a fórmula de [13].

Outro ponto de investigação é a extensão da Torção Analítica para variedades com singularidades. O primeiro espaço a ser considerado é uma variedade com singularidade do tipo cônico. Tal problema foi investigado profundamente por J. Cheeger em vários trabalhos e também outros autores, e já se conhece uma extensão para a teoria de Hodge-de Rham para estes espaços. Logo, surge uma questão lógica a respeito de um Teorema de Cheeger Müller para tais variedades, só que nesta situação estamos fazendo o caminho inverso de Ray e Singer. Apesar de existir muitos resultados da Torção Analítica para tais espaços, a extensão para a Torção de Reidemeister não é bem conhecida. De maneira geral, não se conhece como fixar a base da homologia em uma variedade com singularidade do tipo cônico, ou até mesmo para um cone métrico limitado. No capítulo 4 mostramos que podemos definir, ao menos formalmente, a Torção de Reidemeister para o cone sobre esferas de dimensão qualquer. Seguindo este raciocínio, no capítulo 6, desenvolvemos a Torção Analítica para o cone métrico limitado de uma variedade Riemanniana fechada de dimensão ímpar, mas ainda não apresentamos uma versão apenas com termos geométricos. Para o cone de uma esfera de dimensão ímpar um teorema do tipo Cheeger Müller é provado neste mesmo capítulo. Observamos que B. Vertman [47] determina a Torção Analítica do cone sobre uma variedade Riemanniana fechada qualquer, mas a fórmula obtida é extremamente complicada para uma comparação topológica, diferente da fórmula que obtivemos.

No primeiro capítulo apresentamos as definições e resultados básicos utilizados, como a Teoria de Hodge, ferramentas de Geometria Riemanniana, as variedades com singularidades do tipo cônico e as ferramenta analíticas para o cálculo explícito da Torção Analítica.

Apresentamos no segundo capítulo as definições da Torção de Reidemeister e da Torção Analítica, a motivação de Ray e Singer para a definição da Torção Analítica e os teoremas do tipo Cheeger Müller conhecidos.

No terceiro capítulo é feita a generalização do resultado de W. Luck [27] para variedades com o bordo totalmente geodésico. Determinamos a Torção Analítica da meia-esfera de dimensão arbitrária, utilizando este resultado. No sentido de apresentar uma contribuição quantitativa, fazemos o cálculo da Torção Analítica das calotas esféricas.

No quarto capítulo calculamos a Torção Analítica dos discos de dimensão qualquer usando a fórmula de [4]. Determinamos a Torção de Reidemeister do cone sobre uma esfera de dimensão arbitrária e calculamos diretamente a Torção Analítica do cone sobre a $S^{1}$ e a $S^{2}$.

No capítulo cinco determinamos a Torção Analítica do cone sobre $S^{3}$ e apresentamos a motivação para a generalização do Teorema de Cheeger Müller para as esferas de dimensão ímpar. Este capítulo faz parte do artigo [24].

Por fim, apresentamos a Torção Analítica do cone sobre variedades de dimensão ímpar 
e provamos um teorema do tipo Cheeger Müller para o cone sobre uma esfera de dimensão ímpar. 


\section{Capítulo 1}

\section{Preliminares}

Neste capítulo apresentamos as ferramentas básicas para o desenvolvimento de todo o texto. Observamos que definições e resultados utilizados no decorrer do texto, que não foram definidos ou apresentados, podem ser encontrados nas referências citadas a seguir. Na primeira seção fazemos uma revisão sobre tensores e formas diferenciais, recomendamos para mais informações os livros [33] e [31]. Estas referências servem ainda para as seções 1.2 e 1.3. Recomendamos também uma boa leitura em [17]. Na seção 1.4, para uma leitura mais específica, recomendamos [7], 8], [9], [10] e [34]. Por fim a seção 1.5] é um resumo dos artigos [42], [43], 44] e [46].

\subsection{Tensores e formas diferenciais}

Em alguns momentos no texto usaremos a "soma de Einstein", ou seja, se o mesmo índice aparece duas vezes, sobrescrito e subscrito, isto indica que a soma se dá percorrendo-se todos os valores de tal índice. Por exemplo, se $\mu$ é um índice variando de 1 a $m$, denotamos

$$
A^{\mu} B_{\mu}=\sum_{\mu=1}^{m} A^{\mu} B_{\mu}, \quad A_{\mu} B^{\mu}=\sum_{\mu=1}^{m} A_{\mu} B^{\mu} .
$$

Um vetor dual é um operador linear que aplica um vetor a um escalar. Isto pode ser generalizado para objetos multilineares, chamados tensores, que aplicam vários vetores e vetores duais a um escalar. Um tensor $T$ do tipo $(p, q)$ é uma aplicação multilinear que aplica $p$ vetores duais e $q$ vetores a um número real, ou seja,

$$
T: \underbrace{V^{*} \otimes \cdots \otimes V^{*}}_{p \text { vezes }} \otimes \underbrace{V \otimes \cdots \otimes V}_{q \text { vezes }} \rightarrow \mathbb{R} .
$$

Por exemplo, um tensor do tipo $(0,1)$ aplica um vetor a um número real e pode ser identificado a um vetor dual. 
O conjunto de todos os tensores do tipo $(p, q)$ é chamado o espaço tensor do tipo $(p, q)$ e denotado por $\mathcal{T}_{q}^{p}$. O produto tensorial $\tau=\mu \otimes \nu \in \mathcal{T}_{q}^{p} \otimes \mathcal{T}_{q^{\prime}}^{p^{\prime}}$ é um elemento de $\mathcal{T}_{q+q^{\prime}}^{p+p^{\prime}}$ definido por

$$
\begin{aligned}
\tau\left(\omega_{1}, \ldots, \omega_{p}, \xi_{1}, \ldots, \xi_{p^{\prime}} ; u_{1}, \ldots, u_{q}, v_{1}, \ldots, v_{q^{\prime}}\right) & = \\
& =\mu\left(\omega_{1}, \ldots, \omega_{p} ; u_{1}, \ldots, u_{q}\right) \nu\left(\xi_{1}, \ldots, \xi_{p^{\prime}} ; v_{1}, \ldots, v_{q^{\prime}}\right) .
\end{aligned}
$$

Definição 1. Uma forma diferencial de ordem $r$, ou uma $r$-forma, é um tensor totalmente anti-simétrico do tipo $(0, r)$.

Seja $W$ uma variedade diferenciável de classe $C^{\infty}$. Dado $p \in W$, o espaço tangente $T_{p} W$ é um espaço vetorial cujo o dual é o espaço cotangente $T_{p}^{*} W$. Dessa forma faz sentido considerar formas diferenciáveis em $W$. O exemplo mais simples de uma 1-forma em $W$ é a diferencial $d f$, para $f$ uma função $C^{\infty}$ de $W$ em $\mathbb{R}, d f$ ainda pode ser expresso em termo de coordenadas da seguinte forma: Dada uma vizinhança coordenada $(U, \varphi)$ de $W$ tal que $\varphi(p)=x$, temos

$$
d f=\sum_{\mu} \frac{\partial f}{\partial x_{\mu}} d x_{\mu} .
$$

É natural considerar uma base para $T_{p}^{*} W$ sendo formada por $\left\{d x_{\mu}\right\}$, onde $d x_{\mu}\left(\frac{\partial}{\partial x_{\nu}}\right)=\delta^{\mu}{ }_{\nu}$, onde $\delta^{\mu}{ }_{\nu}$ é o delta de Kronecker.

Definimos o produto wedge $\wedge$ de $r$ 1-formas pelo produto tensorial totalmente antisimétrico

$$
d x_{\mu_{1}} \wedge d x_{\mu_{2}} \wedge \cdots \wedge d x_{\mu_{r}}=\sum_{\sigma \in S_{r}} \operatorname{sgn}(\sigma) d x_{\mu_{\sigma(1)}} \otimes d x_{\mu_{\sigma(2)}} \otimes \cdots \otimes d x_{\mu_{\sigma(r)}}
$$

Se denotarmos o espaço vetorial das $r$-formas em $p \in W$ por $\Omega_{p}^{r}(W)$, o conjunto das $r$-formas (1.1) forma uma base para $\Omega_{p}^{r}(W)$ e um elemento $\omega \in \Omega_{p}^{r}(W)$ é escrito como

$$
\omega=\frac{1}{r !} \sum_{\mu_{1}, \mu_{2}, \ldots, \mu_{r}} \omega_{\mu_{1} \mu_{2} \ldots \mu_{r}} d x_{\mu_{1}} \wedge d x_{\mu_{2}} \wedge \cdots \wedge d x_{\mu_{r}}
$$

onde $\omega_{\mu_{1} \mu_{2} \ldots \mu_{r}}$ são totalmente anti-simétricos, refletindo a anti-simetria da base.

Como existem $\left(\begin{array}{c}m \\ r\end{array}\right)$ escolhas do conjunto $\left(\mu_{1}, \mu_{2}, \ldots, \mu_{r}\right)$, a dimensão do espaço vetorial $\Omega_{p}^{r}(W)$ é

$$
\left(\begin{array}{c}
m \\
r
\end{array}\right)=\frac{m !}{r !(m-r) !} .
$$

Por conveniência definimos $\Omega_{p}^{0}(W)=\mathbb{R}$. Claramente $\Omega_{p}^{1}(W)=T_{p}^{*}(W)$. Se em (1.1) o valor $r$ excede $m$, então o espaço se anula, já que algum índice aparece no mínimo duas vezes no somatório. A igualdade $\left(\begin{array}{c}m \\ r\end{array}\right)=\left(\begin{array}{c}m \\ m-r\end{array}\right)$ implica $\operatorname{dim} \Omega_{p}^{r}(W)=\operatorname{dim} \Omega_{p}^{m-r}(W)$. Como $\Omega_{p}^{r}(W)$ é um espaço vetorial, $\Omega_{p}^{r}(W)$ é isomorfo a $\Omega_{p}^{m-r}(W)$. 
Definimos o produto exterior de uma $q$-forma e uma $r$-forma, $\wedge: \Omega_{p}^{q}(W) \times \Omega_{p}^{r}(W) \rightarrow$ $\Omega_{p}^{q+r}(W)$, por uma extensão trivial. Sejam $\omega \in \Omega_{p}^{q}(W)$ e $\xi \in \Omega_{p}^{r}(W)$. A ação da $(q+r)$-forma $\omega \wedge \xi$ em $q+r$ vetores é definida por

$$
(\omega \wedge \xi)\left(v_{1}, \ldots, v_{q+r}\right)=\frac{1}{q ! r !} \sum_{\sigma \in S_{q+r}} \operatorname{sgn}(\sigma) \omega\left(v_{\sigma(1)}, \ldots, v_{\sigma(q)}\right) \xi\left(v_{\sigma(q+1)}, \ldots, v_{\sigma(q+r)}\right),
$$

onde $v_{i} \in T_{p}(W)$. Se $q+r>m$, então $\omega \wedge \xi$ é identicamente nula. Com este produto definimos uma álgebra (graduada)

$$
\Omega_{p}^{*}(W)=\Omega_{p}^{0}(W) \otimes \Omega_{p}^{1}(W) \otimes \cdots \otimes \Omega_{p}^{m}(W) .
$$

$\Omega_{p}^{*}(W)$ é o espaço de todas as formas diferenciais em $p$ e é fechado pelo produto exterior.

Podemos associar suavemente uma $r$-forma a cada ponto de uma variedade $W$. Denotamos o espaço das $r$-formas suaves em $W$ por $\Omega^{r}(W)$. Também definimos $\Omega^{0}(W)$ sendo a álgebra das funções suaves, $C^{\infty}(W)$. Em resumo, temos a seguinte tabela:

\begin{tabular}{lcc}
\hline$r$-formas & Base & Dimensão \\
\hline$\Omega^{0}(W)=C^{\infty}(W)$ & $\{1\}$ & 1 \\
$\Omega^{1}(W)=T^{*}(W)$ & $\left\{d x_{\mu}\right\}$ & $m$ \\
$\Omega^{2}(W)$ & $\left\{d x_{\mu_{1}} \wedge d x_{\mu_{2}}\right\}$ & $m(m-1) / 2$ \\
\multicolumn{1}{c}{$\vdots$} & $\vdots$ & $\vdots$ \\
$\Omega^{m}(W)$ & $\left\{d x_{1} \wedge d x_{2} \wedge \cdots \wedge d x_{m}\right\}$ & 1 \\
\hline
\end{tabular}

Definição 2. A derivada exterior $d_{r}$ é uma aplicação $d_{r}: \Omega^{r}(W) \rightarrow \Omega^{r+1}(W)$ cuja ação em uma $r$-forma

$$
\omega=\frac{1}{r !} \sum_{\mu_{1}, \mu_{2}, \ldots, \mu_{r}} \omega_{\mu_{1} \mu_{2} \ldots \mu_{r}} d x_{\mu_{1}} \wedge d x_{\mu_{2}} \wedge \cdots \wedge d x_{\mu_{r}}
$$

é definida por

$$
d_{r} \omega=\frac{1}{r !} \sum_{\nu}\left(\sum_{\mu_{1}, \mu_{2}, \ldots, \mu_{r}}\left(\frac{\partial}{\partial x_{\nu}} \omega_{\mu_{1} \ldots \mu_{r}}\right) d x_{\nu} \wedge d x_{\mu_{1}} \wedge d x_{\mu_{2}} \wedge \cdots \wedge d x_{\mu_{r}}\right) .
$$

Quando não houver risco de confusão, omitiremos a dimensão em $d_{r}$ e denotaremos apenas por $d$. Um exemplo da aplicação de $d$ é dado a seguir, se $\omega=\omega_{x}(x, y, z) d x+\omega_{y}(x, y, z) d y+$ $\omega_{z}(x, y, z) d z$ é uma 1-forma em $\mathbb{R}^{3}$ então

$$
\begin{aligned}
d \omega & =\left(\partial_{x} \omega_{y}(x, y, z)-\partial_{y} \omega_{x}(x, y, z)\right) d x \wedge d y+\left(\partial_{y} \omega_{z}(x, y, z)-\partial_{z} \omega_{y}(x, y, z)\right) d y \wedge d z \\
& +\left(\partial_{z} \omega_{x}(x, y, z)-\partial_{x} \omega_{z}(x, y, z)\right) d z \wedge d x
\end{aligned}
$$

Proposição 1. $d^{2}=0\left(\right.$ ou $\left.d_{r+1} d_{r}=0\right)$.

Demonstração. Seja

$$
\omega=\frac{1}{r !} \sum_{\mu_{1}, \mu_{2}, \ldots, \mu_{r}} \omega_{\mu_{1} \mu_{2} \ldots \mu_{r}} d x_{\mu_{1}} \wedge d x_{\mu_{2}} \wedge \cdots \wedge d x_{\mu_{r}} \in \Omega^{r}(W)
$$


A ação de $d^{2}$ em $\omega$ é

$$
d^{2} \omega=\frac{1}{r !} \sum_{\lambda, \nu}\left(\sum_{\mu_{1}, \mu_{2}, \ldots, \mu_{r}}\left(\frac{\partial^{2} \omega_{\mu_{1} \mu_{2} \ldots \mu_{r}}}{\partial x_{\lambda} \partial x_{\nu}}\right) d x_{\lambda} \wedge d x_{\nu} \wedge d x_{\mu_{1}} \wedge d x_{\mu_{2}} \wedge \cdots \wedge d x_{\mu_{r}}\right) .
$$

Assim, $d^{2} \omega$ é identicamente nulo, como $\frac{\partial^{2}}{\partial x_{\lambda} \partial x_{\nu}} \omega_{\mu_{1} \mu_{2} \ldots \mu_{r}}$ é simétrico com relação a $\lambda$ e $\nu$ enquanto que $d x_{\lambda} \wedge d x_{\nu}$ é anti-simétrico.

Seja $N$ uma variedade de classe $C^{\infty}$. Uma aplicação $f: W \rightarrow N$ induz o pullback $f^{*}: T_{f(p)}^{*} N \rightarrow T_{p}^{*}(W)$ que é naturalmente estendido aos tensores do tipo $(0, r)$. Sejam $\omega \in \Omega^{r}(N)$ e $f: W \rightarrow N$ uma aplicação $C^{\infty}$. Em cada ponto $f(p) \in N, f$ induz o pullback $f^{*}: \Omega_{f(p)}^{r}(N) \rightarrow \Omega_{p}^{r}(W)$ dado por

$$
\left(f^{*} \omega\right)\left(X_{1}, \ldots, X_{r}\right) \equiv \omega\left(f_{*} X_{1}, \ldots, f_{*} X_{r}\right)
$$

onde $X_{i} \in T_{p}(W)$ e $f_{*}$ é a aplicação linear $T_{p}(W) \rightarrow T_{f(p)}(N)$.

A derivada exterior $d$ produz a seqüência

$$
0 \longrightarrow \Omega^{0}(W) \stackrel{d_{0}}{\longrightarrow} \Omega^{1}(W) \stackrel{d_{1}}{\longrightarrow} \cdots \stackrel{d_{m-2}}{\longrightarrow} \Omega^{m-1}(W) \stackrel{d_{m-1}}{\longrightarrow} \Omega^{m}(W) \stackrel{d_{m}}{\longrightarrow} 0 .
$$

Esta seqüência é chamada de complexo de de Rham. Como $d^{2}=0$, temos $\operatorname{im} d_{r} \subseteq \operatorname{ker} d_{r+1}$. Um elemento de ker $d_{r}$ é chamado $r$-forma fechada, enquanto um elemento de im $d_{r-1}$ é chamado $r$-forma exata.

Definição 3. O espaço quociente

$$
H^{r}(W, \mathbb{R})=\frac{\operatorname{ker} d_{r}}{\operatorname{im} d_{r-1}}
$$

é chamado de r-ésimo grupo de cohomologia de de Rham.

Para terminar esta seção apresentamos a definição de uma $r$-forma a valores em um espaço vetorial. Seja $V$ um espaço vetorial real $k$ dimensional com base $v_{1}, v_{2}, \ldots, v_{k}$. Lembramos que dado $\omega \in \Omega^{r}(W)$, a cada ponto $p \in W$ associa-se um tensor $\omega_{p}$ da forma

$$
\omega_{p}: T_{p} W \times T_{p} W \cdots \times T_{p} W \rightarrow \mathbb{R}
$$

que varia diferencialmente com respeito a $p$. Podemos definir uma $r$-forma com valores em $V$ trocando $\mathbb{R}$ por $V$ na equação anterior de maneira que, se $\omega=\sum_{i=1}^{k} \omega_{i}$, onde $\omega_{i}$ são $r$-formas, então $\omega$ passa a ser escrita da seguinte forma

$$
\omega=\sum_{i=1}^{k} \omega_{i} \otimes v_{i}
$$

Denotaremos o espaço destas formas por $\Omega^{r}(W ; V)$. Toda a teoria apresentada nesta seção e na seção 1.3 generaliza-se para este caso sem maiores problemas. 


\subsection{Geometria Riemanniana}

Seja $W$ uma variedade de classe $C^{\infty}$ e dimensão $m$.

Definição 4. Uma métrica Riemanniana $g$ em $W$ é um tensor do tipo (0,2) em $W$ que associa, de maneira continua, para cada ponto $p \in W$ um produto interno $g_{p}$ em $T_{p} W$, ou seja, dados $u, v \in T_{p}(W)$,

1. $g_{p}(u, v)=g_{p}(v, u)$,

2. $g_{p}(u, u) \geq 0$, valendo a igualdade se, e somente se, $u=0$.

O par $(W, g)$ é chamado de variedade Riemanniana.

Sejam $(U, \phi)$ uma vizinhança coordenada em $p \in W$ tal que $\phi(p)=x$ e $\left\{x_{\mu}\right\}$ as coordenadas. Já que $g \in \mathcal{T}_{2}^{0}(W), g$ é expandida em termos de $d x_{\mu} \otimes d x_{\nu}$ da forma

$$
g_{p}=\sum_{\mu, \nu} g_{\mu \nu}(p) d x_{\mu} \otimes d x_{\nu}
$$

onde

$$
g_{\mu \nu}(p)=g_{p}\left(\frac{\partial}{\partial x_{\mu}}, \frac{\partial}{\partial x_{\nu}}\right)=g_{\nu \mu}(p) .
$$

Do mesmo modo que procedemos com o operador $d$ omitiremos $p$ em $g_{\mu \nu}$, a menos que isto possa causar confusão. É comum tratar $\left(g_{\mu \nu}\right)$ como uma matriz cuja entrada $(\mu, \nu)$ (linha $\mu$ coluna $\nu)$ é $g_{\mu \nu}$. Desta forma $\left(g_{\mu \nu}\right)$ tem posto máximo e inversa denotada por $\left(g^{\mu \nu}\right)$, satisfazendo $g_{\mu \nu} g^{\nu \lambda}=g^{\lambda \nu} g_{\nu \mu}=\delta^{\mu}{ }_{\lambda}$, onde a entrada da forma $A_{b}^{a}$ significa o elemento da matriz $A$ da linha $a$ e coluna $b$ e $A_{a}{ }^{b}$ é a coluna $a$ e a linha $b$ da matriz inversa de $A$, quando $A$ possuir inversa. O determinante $\operatorname{det}\left(g_{\mu \nu}\right)$ é denotado por $g$ e $\operatorname{det}\left(g^{\mu \nu}\right)=g^{-1}$. Temos ainda que a matriz $\left(g_{\mu \nu}\right)$ é real e simétrica com autovalores reais e todos positivos.

Seja $N$ uma subvariedade de dimensão $n$ de uma variedade Riemanniana $W$ de dimensão $m$, com uma métrica $g_{W}$. Se $f: N \rightarrow W$ é um mergulho que induz a estrutura de subvariedade em $N$, o pullback $f^{*}$ induz a métrica natural $g_{N}=f^{*} g_{W}$ em $N$. As componentes de $g_{N}$ são dadas por

$$
g_{N \mu \nu}(x)=\sum_{\alpha, \beta} g_{W \alpha \beta}(f(x)) \frac{\partial f_{\alpha}}{\partial x_{\mu}} \frac{\partial f_{\beta}}{\partial x_{\nu}},
$$

onde $f_{\alpha}$ denota as coordenadas de $f(x)$. Por exemplo, considere a métrica da esfera unitária mergulhada em $\left(\mathbb{R}^{3}, g_{E}\right)$, onde $g_{E}$ é a métrica euclidiana. Seja $(\theta, \phi)$ a coordenada polar de $S^{2}$ e defina $f$ pela inclusão usual

$$
f:(\theta, \phi) \mapsto(\operatorname{sen} \theta \cos \phi, \operatorname{sen} \theta \operatorname{sen} \phi, \cos \theta),
$$

da qual obtemos a métrica induzida

$$
\begin{aligned}
g_{S^{2}}=\sum_{\mu, \nu} g_{\mu \nu} d x_{\mu} \otimes d x_{\nu} & =\sum_{\mu, \nu, \alpha, \beta} g_{E \alpha \beta} \frac{\partial f_{\alpha}}{\partial x_{\mu}} \frac{\partial f_{\beta}}{\partial x_{\nu}} d x_{\mu} \otimes d x_{\nu} \\
& =d \theta \otimes d \theta+(\operatorname{sen} \theta)^{2} d \phi \otimes d \phi .
\end{aligned}
$$


Faremos uma construção local para definir a 1-forma conexão e a 2-forma curvatura de um variedade Riemanniana $W$. Dado $p \in W$ considere uma base coordenada para $T_{p} W$ formada por $\left\{\partial / \partial x_{\mu}\right\}$ e para $T_{p}^{*} W$ formada por $\left\{d x_{\mu}\right\}$. Como $W$ é Riemanniana, podemos ortonormalizar a base $\left\{\partial / \partial x_{\mu}\right\}$ de maneira que

$$
e_{\alpha}=e_{\alpha}{ }^{\mu} \frac{\partial}{\partial x_{\mu}}
$$

com det $e_{\alpha}^{\mu}>0$ e $g\left(e_{\alpha}, e_{\beta}\right)=e_{\alpha}{ }^{\mu} e_{\beta}{ }^{\nu} g_{\mu \nu}=\delta_{\alpha}{ }^{\beta}$, ou seja, $\left\{e_{\alpha}\right\}$ é uma base ortonormal.

Lembrando o produto interno natural entre um vetor $v \in T_{p}(W)$ e um vetor dual $\omega \in$ $T_{p}^{*}(W)$,

$$
\langle,\rangle: T_{p}^{*}(W) \times T_{p}(W) \rightarrow \mathbb{R},\langle\omega, v\rangle=\omega(v)
$$

definimos a base dual $\left\{e^{\alpha}\right\}$ de maneira que $\left\langle e^{\alpha}, e_{\beta}\right\rangle=\delta_{\alpha}{ }^{\beta}$. Desta forma $e^{\alpha}$ é dado por

$$
e^{\alpha}=\sum_{\mu} e_{\mu}^{\alpha} d x_{\mu}
$$

Observamos que a matriz $\left(e^{\alpha}{ }_{\mu}\right)$ é a matriz inversa de $\left(e_{\alpha}{ }^{\mu}\right)$, ou seja, $e^{\alpha}{ }_{\mu} e_{\alpha}{ }^{\nu}=\delta_{\mu}{ }^{\nu}$ e $e^{\alpha}{ }_{\mu} e_{\beta}{ }^{\mu}=$ $\delta_{\beta}^{\alpha}$. Em termos de $\left\{e^{\alpha}\right\}$, a métrica $g$ tem a forma

$$
g=\sum_{\mu, \nu} g_{\mu \nu} d x_{\mu} \otimes d x_{\nu}=\delta_{\alpha \beta} e^{\alpha} \otimes e^{\beta} .
$$

As bases $\left\{e_{\alpha}\right\}$ e $\left\{e^{\alpha}\right\}$ são chamadas de bases não coordenadas. A base não coordenada possui o colchete de Lie não nulo, ou seja,

$$
\left.\left[e_{\alpha}, e_{\beta}\right]\right|_{p}=\left.c_{\alpha \beta}^{\gamma}(p) e_{\gamma}\right|_{p}
$$

onde

$$
c_{\alpha \beta}^{\gamma}(p)=e_{\nu}^{\gamma}\left(e_{\alpha}{ }^{\mu} \partial_{\mu} e_{\beta}{ }^{\nu}-e_{\beta}{ }^{\mu} \partial_{\mu} e_{\alpha}{ }^{\nu}\right)(p),
$$

os símbolos $c_{\alpha \beta}^{\gamma}$ são chamados de constantes de Cartan. Como fizemos anteriormente, omitiremos $p$ das fórmulas sempre que não houver risco de confusão.

Defina os coeficientes da conexão com respeito a base $\left\{e_{\alpha}\right\}$ por

$$
\nabla_{e_{\alpha}} e_{\beta}=\Gamma_{\alpha \beta}^{\gamma} e_{\gamma}
$$

onde $\nabla$ é a conexão Riemanniana associada a métrica. Observamos que o coeficiente de Christoffel que aparece na equação acima é associado a base não coordenada; não o devemos confundir com o coeficiente de Christoffel dado pela conexão na base coordenada. Para determinar $\Gamma_{\alpha \beta}^{\gamma}$ temos a fórmula

$$
\Gamma_{\alpha \beta}^{\gamma}=\frac{c_{\alpha \beta}^{\gamma}+c_{\gamma \alpha}{ }^{\beta}+c_{\gamma \beta}{ }^{\alpha}}{2} .
$$


Definição 5. A matriz com valores em 1-formas $\omega=\omega_{\beta}^{\alpha}$ é chamada de 1-forma conexão $e$ dada por

$$
\omega_{\beta}^{\alpha}=\Gamma_{\gamma}^{\alpha}{ }_{\beta} e^{\gamma} .
$$

A matriz com valores em 2-formas $R=R^{\alpha}{ }_{\beta}$ é chamada de 2-forma curvatura e dada por

$$
R_{\beta}^{\alpha}=d \omega_{\beta}^{\alpha}+\omega_{\gamma}^{\alpha} \wedge \omega_{\beta}^{\gamma} \text {. }
$$

Seja $A=\left(a^{i}\right)$ uma matriz anti-simétrica $\left(A^{t}=-A\right)$ de dimensão $2 k$, então o Pfaffiano de $A$ é dado por

$$
\operatorname{Pf}(A)=\frac{1}{2^{k} k !} \sum_{\sigma \in S_{2 k}} \operatorname{sgn} \sigma a_{\sigma(2)}^{\sigma(1)} a_{\sigma(4)}^{\sigma(3)} \ldots a^{\sigma(2 k-1)} \sigma(2 k),
$$

onde $S_{2 k}$ é o grupo de permutações de dimensão $2 k$.

Definição 6. A classe de Euler $e(W, g)$ é definida como

$$
e(W, g)=\frac{1}{(2 \pi)^{m}} \operatorname{Pf}(R)
$$

onde $R=R_{\beta}^{\alpha}$ é a 2-forma curvatura de $W$, se a dimensão de $W$ é par e igual a $2 \mathrm{~m}$. Se a dimensão de $W$ é impar, definimos $e(W, g)=0$.

Teorema 1 (Teorema de Gauss-Bonet). Seja $W$ uma variedade Riemanniana orientada fechada de dimensão par, e classe $C^{\infty}$, então

$$
\int_{W} e(W, g)=\chi(W)
$$

Para terminar a seção definimos o que significa uma curva parametrizada $f: I \rightarrow W$ ser uma geodésica de $W$ e apresentamos as equações locais satisfeitas por uma geodésica. Seja $I \subset \mathbb{R}$ um intervalo aberto.

Definição 7. Uma curva parametrizada $f: I \rightarrow W$ é dita uma geodésica de $W$ se para todo $t \in I, \frac{D}{d t}\left(\partial_{t} f\right)=0$, onde $\frac{D}{d t}\left(\partial_{t} f\right)$ denota a derivada covariante de $\partial_{t} f$ ao longo de $f$.

Se $[a, b] \subset I$, então a restrição de $f$ a $[a, b]$ é chamada de (segmento de) geodésica ligando $f(a)$ a $f(b)$.

Vamos determinar as equações locais satisfeitas por uma geodésica $f$ em um sistemas de coordenadas $(U, \phi)$ em torno de $f\left(t_{0}\right), t_{0} \in I$ tal que $\phi\left(f\left(t_{0}\right)\right)=x$. Suponha que $f(I) \subset U$, então $f$ será uma geodésica se e só se

$$
0=\frac{D}{d t}\left(\partial_{t} f\right)=\sum_{k=1}^{m}\left(\partial_{t}^{2} f^{x_{k}}+\Gamma_{\alpha}{ }^{k}{ }_{\beta} \partial_{t} f^{x_{\alpha}} \partial_{t} f^{x_{\beta}}\right) \frac{\partial}{\partial x_{k}} .
$$

Logo $f$ é uma geodésica se, e somente se, satisfaz

$$
\partial_{t}^{2} f^{x_{k}}+\Gamma_{\alpha}{ }^{k} \partial_{t} f^{x_{\alpha}} \partial_{t} f^{x_{\beta}}=0,
$$

para $k=1, \ldots, m$. Esta equação é chamada de equação geodésica em $W$. 


\subsection{Teoria de Hodge}

Se $W$ é uma variedade Riemanniana orientável de dimensão $m$ e classe $C^{\infty}$, existe uma $m$ forma $\omega$ que não se anula em ponto algum. Esta $m$-forma é chamada de elemento volume (ou forma de volume) e faz o papel de uma medida quando integramos uma função $f \in C^{\infty}(W)$ sobre $W$. Se $W$ possui uma métrica $g$, existe um elemento volume natural que é invariante por transformações coordenadas. Definimos o elemento volume invariante por

$$
\Omega_{W}=\sqrt{|\operatorname{det} g|} d x_{1} \wedge d x_{2} \wedge \cdots \wedge d x_{m}
$$

onde $\left\{x_{\mu}\right\}$ são as coordenadas de $(U, \phi)$.

Como observado anteriormente, $\Omega^{r}(W)$ é isomorfo a $\Omega^{m-r}(W)$. Utilizaremos a métrica $g$ de $W$ para definir um isomorfismo entre $\Omega^{r}(W)$ e $\Omega^{m-r}(W)$, chamado $*$ de Hodge. Definimos o tensor totalmente anti-simétrico $\varepsilon$ por

$$
\varepsilon_{\mu_{1} \mu_{2} \ldots \mu_{m}}=\left\{\begin{array}{cl}
+1 & \text { se }\left(\mu_{1} \mu_{2} \ldots \mu_{m}\right) \text { é uma permutação par de }(12 \ldots m), \\
-1 & \text { se }\left(\mu_{1} \mu_{2} \ldots \mu_{m}\right) \text { é uma permutação ímpar de }(12 \ldots m), \\
0 & \text { caso contrário. }
\end{array}\right.
$$

Note que $\varepsilon^{\mu_{1} \mu_{2} \ldots \mu_{m}}=g^{\mu_{1} \nu_{1}} g^{\mu_{2} \nu_{2}} \cdots g^{\mu_{m} \nu_{m}} \varepsilon_{\nu_{1} \nu_{2} \ldots \nu_{m}}=g^{-1} \varepsilon_{\mu_{1} \mu_{2} \ldots \mu_{m}}$. Observamos que para "subir" ou "baixar" os índices dos tensores usaremos a métrica, por exemplo, $\varepsilon_{i k l}=g_{i j} \varepsilon^{j}{ }_{k l}$.

A * de Hodge, chamado também de operador estrela de Hodge, é uma aplicação linear * : $\Omega^{r}(W) \rightarrow \Omega^{m-r}(W)$ cuja ação em um vetor da base de $\Omega^{r}(W)$ é definida por

$$
*\left(d x_{\mu_{1}} \wedge \cdots \wedge d x_{\mu_{r}}\right)=\frac{\sqrt{|g|}}{(m-r) !} \sum_{\nu_{r+1}, \ldots, \nu_{m}} \varepsilon_{\nu_{r+1} \ldots \nu_{m}}^{\mu_{1} \ldots \mu_{r_{r}}} d x_{\nu_{r+1}} \wedge \cdots \wedge d x_{\nu_{m}} .
$$

Notemos que $*(1)$ é o elemento volume invariante

$$
*(1)=\frac{\sqrt{|g|}}{m !} \sum_{\mu_{1}, \ldots, \mu_{m}} \varepsilon_{\mu_{1} \ldots \mu_{m}} d x_{\mu_{1}} \wedge \cdots \wedge d x_{\mu_{m}}=\sqrt{|g|} d x_{1} \wedge \cdots \wedge d x_{m} .
$$

Para

$$
\omega=\frac{1}{r !} \sum_{\mu_{1}, \ldots, \mu_{r}} \omega_{\mu_{1} \mu_{2} \ldots \mu_{r}} d x_{\mu_{1}} \wedge d x_{\mu_{2}} \wedge \cdots \wedge d x_{\mu_{r}} \in \Omega^{r}(W)
$$

temos

$$
*(\omega)=\frac{\sqrt{|g|}}{r !(m-r) !} \sum_{\nu_{r+1}, \ldots, \nu_{m}} \omega_{\mu_{1} \ldots \mu_{r}} \varepsilon_{\nu_{r+1} \ldots \nu_{m}}^{\mu_{1} \ldots \mu_{r}} d x_{\nu_{r+1}} \wedge \cdots \wedge d x_{\nu_{m}} .
$$

Logo $* *(\omega)=(-1)^{r(m-r)} \omega$. Desta forma, definimos a inversa de $*$ por $*^{-1}=(-1)^{r(m-r)} *$.

Sejam $\omega, \eta \in \Omega^{r}(W)$ dadas por

$$
\omega=\frac{1}{r !} \sum_{\mu_{1}, \ldots, \mu_{r}} \omega_{\mu_{1} \mu_{2} \ldots \mu_{r}} d x_{\mu_{1}} \wedge d x_{\mu_{2}} \wedge \cdots \wedge d x_{\mu_{r}}
$$




$$
\eta=\frac{1}{r !} \sum_{\nu_{1}, \ldots, \nu_{r}} \eta_{\nu_{1} \nu_{2} \ldots \nu_{r}} d x_{\nu_{1}} \wedge d x_{\nu_{2}} \wedge \cdots \wedge d x_{\nu_{r}} .
$$

Observamos que $\omega \wedge * \eta$ é uma $m$-forma,

$$
\begin{aligned}
\omega \wedge * \eta & =\frac{1}{(r !)^{2}} \sum_{\mu_{1}, \ldots, \mu_{r}} \omega_{\mu_{1} \ldots \mu_{r}} \eta_{\nu_{1} \ldots \nu_{r}} \frac{\sqrt{|g|}}{(m-r) !} \varepsilon^{\nu_{1} \ldots \nu_{r}}{ }_{\mu_{r+1} \ldots \mu_{m}} d x_{\mu_{1}} \wedge \cdots \wedge d x_{\mu_{r}} \wedge \cdots \wedge d x_{\mu_{m}} \\
& =\frac{1}{r !} \sum_{\mu_{1}, \ldots, \mu_{r}} \omega_{\mu_{1} \ldots \mu_{r}} \eta^{\nu_{1} \ldots \nu_{r}} \frac{1}{r !(m-r) !} \varepsilon_{\nu_{1} \ldots \nu_{r} \mu_{r+1} \ldots \mu_{m}} \varepsilon_{\mu_{1} \ldots \mu_{r} \mu_{r+1} \ldots \mu_{m}} \sqrt{|g|} d x_{1} \wedge \cdots \wedge d x_{m} \\
& =\frac{1}{r !} \omega_{\mu_{1} \ldots \mu_{r}} \eta^{\mu_{1} \ldots \mu_{r}} \sqrt{|g|} d x_{1} \wedge \cdots \wedge d x_{m} .
\end{aligned}
$$

$\mathrm{E} \operatorname{assim} \omega \wedge * \eta=\eta \wedge * \omega$. Como $\omega \wedge * \eta$ é uma $m$-forma, sua integral sobre $W$ está bem definida. Definimos o produto interno $(\omega, \eta)$ de duas $r$-formas por

$$
(\omega, \eta)=\int \omega \wedge * \eta=\frac{1}{r !} \int_{W} \omega_{\mu_{1} \ldots \mu_{r}} \eta^{\mu_{1} \ldots \mu_{r}} \sqrt{|g|} d x_{1} \wedge \ldots \wedge d x_{m} .
$$

Desta forma este produto interno é simétrico, $(\omega, \eta)=(\eta, \omega)$ e ainda, como $(W, g)$ é Riemanniana, o produto interno é positivo definido.

Definição 8. O operador adjunto formal da derivada exterior é $d^{\dagger}: \Omega^{r}(W) \rightarrow \Omega^{r-1}(W)$ definido por

$$
d^{\dagger}=(-1)^{m r+m+1} * d * .
$$

Um elemento de ker $d^{\dagger}$ será chamado de $r$-forma cofechada e um elemento de im $d^{\dagger}$ será chamado de $r$-forma coexata. Observamos que $\left(d^{\dagger}\right)^{2}=0$ como $d^{2}=0$.

Definição 9. O operador

$$
\Delta^{(r)}=\left(d+d^{\dagger}\right)^{2}=d_{r+1}^{\dagger} d_{r}+d_{r-1} d_{r}^{\dagger}: \Omega^{r}(W) \rightarrow \Omega^{r}(W),
$$

é chamado Laplaciano.

Observamos que $d \Delta=\Delta d$ e $\Delta d^{\dagger}=d^{\dagger} \Delta$. Ainda mais, o Laplaciano $\Delta$ é um operador positivo em $W$, ou seja,

$$
(\omega, \Delta \omega)=\left(\omega,\left(d d^{\dagger}+d^{\dagger} d\right) \omega\right)=(d \omega, d \omega)+\left(d^{\dagger} \omega, d^{\dagger} \omega\right) \geq 0 .
$$

Uma forma $\omega$ é chamada harmônica se $\Delta \omega=0$. Denotamos o conjunto das $r$-formas harmônicas em $W$ por $\mathcal{H}^{r}(W)$. Como conseqüência imediata de (1.8), temos:

Proposição 2. Uma forma $\omega$ sobre uma variedade Riemanniana compacta é harmônica se, e somente se, $\omega$ é fechada e cofechada.

Teorema 2 (Decomposição de Hodge). Seja $(W, g)$ uma variedade Riemanniana orientável compacta sem bordo. Então $\Omega^{r}(W)$ é unicamente decomposto como

$$
\Omega^{r}(W)=\mathcal{H}^{r}(W) \oplus d_{r-1} \Omega^{r-1}(W) \oplus d_{r+1}^{\dagger} \Omega^{r+1}(W) .
$$

Para a prova do teorema anterior ver [31. 


\subsection{Variedades com singularidades do tipo cônico}

Apresentaremos a definição de variedades com singularidades do tipo cônico e resultados relacionados a teoria de Hodge, mais precisamente, faremos a extensão do operador Laplaciano para este tipo de espaço. A maioria das provas serão apenas indicadas, de maneira que os interessados possam se aprofundar ainda mais no assunto. Os artigos básicos utilizados nesta seção são [5], 8], [9], [10] e [34] .

Seja $N$ uma variedade Riemanniana $m$-dimensional fechada (podendo ser conexa ou não) com métrica $\tilde{g}$.

Definição 10. Um cone métrico $C N$ é o espaço $(0, \infty) \times N$, com a métrica

$$
g=d r \otimes d r+r^{2} \tilde{g}
$$

Definimos também um cone métrico limitado $C_{l} N$ em $N$ sendo o espaço $(0, l] \times N$ com a métrica dada em (1.9).

Definição 11. $X^{m+1}$ é uma variedade Riemanniana com singularidade do tipo cônico se existem $p_{j} \in X^{m+1}, j=1, \ldots, k$, tal que $X^{m+1}-\cup_{j=1}^{k} p_{j}$ é uma variedade Riemanniana suave aberta (possivelmente com bordo) e cada $p_{j}$ possui um vizinhança $U_{j}$ tal que $U_{j}-\left\{p_{j}\right\}$ é isométrico a $C_{l_{j}} N_{j}^{m}$ para algum $l_{j} \in \mathbb{R}$ e alguma $N_{j}^{m}$.

Observamos que fazer a análise em $X^{m+1}$ significa fazer a análise na variedade Riemanniana incompleta $X^{m+1}-\cup_{j=1}^{k} p_{j}$. Para um exemplo simples podemos assumir $k=1$ e $l=1$ e escrevemos $X=C_{1} N \cup M$, onde $\partial M=N$ e a união é feita pelo bordo. Um esboço de tal fato é a figura abaixo.

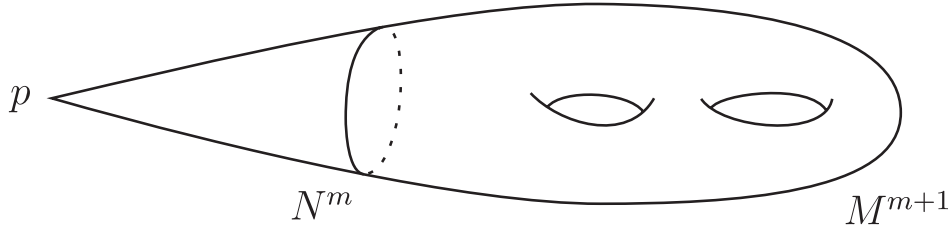

Figura 1.1: Exemplo de uma variedade com singularidade do tipo cônico.

Existe ao menos um caso em que $C_{l} N$ não possui singularidade; isto ocorre quando $N$ é a esfera de raio 1 e assim $C_{l} S_{1}^{n}=D_{l}^{n+1}$. Por outro lado, se $N$ é uma esfera de raio $r \neq 1$ então o vértice do cone $C_{l} N$ é uma singularidade. Este é o exemplo mais simples em que se tem uma singularidade.

Um espaço que é uma variedade Riemanniana com singularidade do tipo cônico foi bem estudado por J. Cheeger que estendeu a teoria de Hodge para este tipo de espaço considerando toda a teoria com a cohomologia $L^{2}$. Tal teoria é construída de maneira análoga a que foi apresentada anteriormente só que considera-se apenas formas quadrado integráveis. Com isto é 
possível definir a Torção Analítica para estes espaços da mesma maneira que está no capítulo 2. considerando as formas em $L^{2}$ e a extensão do Laplaciano da maneira que é discutida abaixo. Já no caso da Torção de Reidemeister não temos uma definição para estes espaços. Observamos que a cohomologia $L^{2}$ de uma variedade com singularidade do tipo cônico $X$ coincide com a cohomologia usual de $X$ quando $X$ é uma variedade homológica racional, ou seja, se para cada $x \in X$ o grupo de homologia local $H_{q}(X, X-x)$ é zero se $q \neq m+1$ e $\mathbb{Q}$ se $q=m+1$.

Com respeito ao operador Laplaciano sobre $C_{l} N$ alguns cuidados são necessários. Decompondo com respeito as projeções sobre os autoespaços da restrição do Laplaciano sobre a seção do cone, a definição de uma extensão auto-adjunta apropriada do operador Laplaciano (sobre as funções) em $C_{l} N$ reduz-se a análise dos valores de contorno de uma equação diferencial ordinal de segunda ordem de Sturm Liouville no intervalo $(0, l]$. Tal problema já foi resolvido por Rellich em [39], que parametrizou as extensões auto-adjuntas. Em particular, significa que não existem condições de contorno para os casos em que $r \in(0, l)$, enquanto condições de contorno em $r=0, l$ são necessárias. A única extensão auto-adjunta definida por estas condições de contorno é a extensão máxima, correspondente a extensão de Friedrich (ver [5] ou [9], para as condições de contorno). O mesmo argumento vale para o Laplaciano nas formas.

Seja $C_{l} N$ como anteriormente, assumimos coordenadas para $C_{l} N$ como sendo $(r, y)$, onde $r \in(0, l]$ e $y \in N$. Operações na seção serão indicadas com til, por exemplo $\tilde{\Delta}$ é o Laplaciano em $N$. A demonstração do próximo lema é pura e simples aplicação das definições e persistência nos cálculos, não faremos a prova aqui. Aos interessados ver [7](com alguns erros de digitação) e [34].

Lema 1. Seja $\theta(r, y)=g(r) \phi(y)+f(r) d r \wedge \omega(y)$ uma q-forma em $C_{l} N$, onde $f, g$ são funções suaves em $r \in(0, l)$ e $\phi, \omega$ são q e $(q-1)$ formas suaves em $N$. Então temos:

$$
\begin{aligned}
* \theta(r, y) & =f(r) r^{m-2 q+2} \tilde{*} \omega(y)+(-1)^{q} r^{m-2 q} g(r) d r \wedge \tilde{*} \phi(y) \\
d \theta(r, y) & =g(r) \tilde{d} \phi(y)+\partial_{r} g(r) d r \wedge \phi(y)-f(r) d r \wedge d \omega(y) \\
d^{\dagger} \theta(r, y) & =r^{-2} g(r) \tilde{d}^{\dagger} \phi(y)-\left((m-2 q+2) r^{-1} f(r)+\partial_{r} f(r)\right) \omega(y)-r^{-2} f(r) d r \wedge \tilde{d}^{\dagger} \omega(y) \\
\Delta(\theta(r, y)) & =\left(-\partial_{r}^{2} g(r)-(m-2 q) r^{-1} \partial_{r} g(r)\right) \phi(y)+r^{-2} g(r) \tilde{\Delta}(\phi(y))-2 r^{-1} f(r) \tilde{d} \omega(y) \\
& +d r \wedge\left(r^{-2} f(r) \tilde{\Delta} \omega(y)+\omega(y)\left(-\partial_{r}^{2} f(r)-(m-2 q+2) r^{-1} \partial_{r} f\right.\right. \\
& \left.\left.+(m-2 q+2) r^{-2} f(r)\right)-2 r^{-3} g(r) \tilde{d}^{\dagger} \phi(y)\right) .
\end{aligned}
$$

Um dos nossos objetivos no capítulo [6 é determinar o espectro do operador Laplaciano sobre as formas do cone. Como $C_{l} N$ não é compacto, é necessário definir o domínio do operador concreto $\Delta^{(q)}$, no sentido de obter a extensão auto-adjunta correta. Para isto, usaremos o lema 2. Depois, encontramos todas as soluções da equação dos autovalores. Observamos que a prova do lema 2 usa ferramentas que fogem da nossa abordagem e não 
será feita. Para a prova ver [5]. Observamos que a definição das condições de contorno absoluta (relativa) no bordo está no próximo capítulo.

Lema 2. O operador formal $\Delta^{(q)}$ do lema 1 com condições de contorno absoluta (relativa) no bordo $\partial C_{l} N$ define um único operador auto-adjunto semi-limitado em $L^{2}\left(\Omega^{(q)}\left(C_{l} N\right)\right)$, que denotaremos por $\Delta_{\text {abs }}^{(q)}\left(\Delta_{\text {rel }}^{(q)}\right)$ com um espectro pontual.

Com o lema anterior passamos ao estudo específico de $\Delta^{(q)}$. Defina os números

$$
\alpha_{q}=\frac{1}{2}(1+2 q-m), \mu_{q, n}=\sqrt{\lambda_{q, n}+\alpha_{q}^{2}},
$$

onde $\lambda_{q, n}$ são autovalores de autoformas coexatas $\phi_{n}^{(q)}$ de $\tilde{\Delta}^{(q)}$ de dimensão $q$ na seção, com multiplicidade $m_{q, n}$. Note que qualquer forma em $C_{l} N$ pode ser escrita da forma $\theta(r, y)$ como no lema 1 Em [9], Jeff Cheeger fez o estudo do caso que $\Delta(\theta(r, y))=\lambda^{2} \theta(r, y) \operatorname{com} \lambda \neq 0$ e quando $\lambda=0$. Faremos um pequeno resumo sobre o caso em que $\lambda \neq 0$.

Seja $\theta(r, y)$ uma $q$ forma em $C_{l} N$, escrita da maneira do lema 1 , e $\lambda \neq 0$ tal que $\Delta(\theta(r, y))=$ $\lambda^{2} \theta(r, y)$ então

$$
\begin{aligned}
& \Delta(g(r) \phi(y)+f(r) d r \wedge \omega(y))=\lambda^{2} g(r) \phi(y)+\lambda^{2} f(r) d r \wedge \omega(y) \Rightarrow \\
& \left\{\begin{array}{l}
\left(-\partial_{r}^{2} g-(m-2 q) r^{-1} \partial_{r} g-\lambda^{2} g\right) \phi+r^{-2} g \tilde{\Delta}(\phi)-2 r^{-1} f \tilde{d} \omega=0, \\
d r \wedge\left(r^{-2} f \tilde{\Delta} \omega+\omega\left(-\partial_{r}^{2} f-(m-2 q+2) r^{-1} \partial_{r} f\right.\right. \\
\left.\left.+(m-2 q+2) r^{-2} f-\lambda^{2} f\right)-2 r^{-3} g \tilde{d}^{\dagger} \phi\right)=0,
\end{array}\right.
\end{aligned}
$$

onde, por questão de espaço, omitimos $r$ e $y$ das funções e formas. Usando o teorema da decomposição de Hodge (teorema 21), segue-se que as formas $\phi \in \Omega^{(q)}(N)$ e $\omega \in \Omega^{(q-1)}(N)$ se decompõem em uma combinação entre formas harmônicas, exatas e coexatas. Com esta decomposição, substituindo no sistema acima, vemos que as soluções são combinações das formas do teorema abaixo,

Teorema 3. Considere $\mu_{q, n}$ e $\alpha_{q}$ como acima. Sejam $\phi_{n}^{(q-2)}, \phi_{n}^{(q-1)}$ e $\phi_{n}^{(q)}$ autoformas coexatas de $\tilde{\Delta}$ e $h^{(q-1)}, h^{(q)}$ formas harmônicas de $\tilde{\Delta}$. Então as autoformas de $\Delta^{(q)}$ com autovalores não nulos são:

$$
\begin{aligned}
& \psi_{1, n}^{(q)}=r^{\alpha_{q}} J_{\mu_{q, n}}(\lambda r) \phi_{n}^{(q)} \\
& \psi_{2, n}^{(q)}=r^{\alpha_{q-1}} J_{\mu_{q-1, n}}(\lambda r) \tilde{d} \phi_{n}^{(q-1)}+\partial_{r}\left(r^{\alpha_{q-1}} J_{\mu_{q-1, n}}(\lambda r)\right) d r \wedge \phi_{n}^{(q-1)} \\
& \psi_{3, n}^{(q)}=r^{2 \alpha_{q-1}+1} \partial_{r}\left(r^{-\alpha_{q-1}} J_{\mu_{q-1, n}}(\lambda r)\right) \tilde{d} \phi_{n}^{(q-1)}+r^{\alpha_{q-1}-1} J_{\mu_{q-1, n}}(\lambda r) d r \wedge \tilde{d}^{\dagger} \tilde{d} \phi_{n}^{(q-1)} \\
& \psi_{4, n}^{(q)}=r^{\alpha_{q-2}+1} J_{\mu_{q-2, n}}(\lambda r) d r \wedge \tilde{d} \phi_{n}^{(q-2)} \\
& \psi_{E}^{(q)}=r^{\alpha_{q}} J_{\left|\alpha_{q}\right|}(\lambda r) h^{(q)} \\
& \psi_{O}^{(q)}=\partial_{r}\left(r^{\alpha_{q-1}} J_{\left|\alpha_{q-1}\right|}(\lambda r)\right) d r \wedge h^{(q-1)}
\end{aligned}
$$

Chamaremos tais formas respectivamente por tipo 1, 2, 3, 4, E e O. Quando $\mu_{q, n}$ é metade de um inteiro as soluções (-) devem ser modificadas incluindo termos logarítmicos (ver [21] ou [48], para um conjunto linearmente independente de soluções para equações de Bessel). 
Demonstração. A prova é uma verificação direta da afirmação. Primeiro, decompomos qualquer forma $\omega \in \Omega^{q}(N)$ em uma combinação entre formas harmônicas, exatas e coexatas. Segundo, Substituindo a forma no sistema do Laplaciano obtemos equações diferenciais das funções que aparecem como coeficientes das formas. Todas estas equações são reduzidas a equações de Bessel. Depois, escrevemos todas as soluções usando funções de Bessel, e obtemos

$$
\begin{aligned}
\psi_{ \pm 1, n, \lambda}^{(q)} & =r^{\alpha_{q}} J_{ \pm \mu_{q, n}}(\lambda r) \phi_{n}^{(q)} \\
\psi_{ \pm 2, n, \lambda}^{(q)} & =r^{\alpha_{q-1}} J_{ \pm \mu_{q-1, n}}(\lambda r) \tilde{d} \phi^{(q-1)}+\partial_{r}\left(r^{\alpha_{q-1}} J_{ \pm \mu_{q-1, n}}(\lambda r)\right) d r \wedge \phi^{(q-1)} \\
\psi_{ \pm, 3, n, \lambda}^{(q)} & =r^{2 \alpha_{q-1}+1} \partial_{r}\left(r^{-\alpha_{q-1}} J_{ \pm \mu_{q-1, n}}(\lambda r)\right) \tilde{d} \phi^{(q-1)}+r^{\alpha_{q-1}-1} J_{ \pm \mu_{q-1, n}}(\lambda r) d r \wedge \tilde{d}^{\dagger} \tilde{d} \phi^{(q)} \\
\psi_{ \pm, 4, n, \lambda}^{(q)} & =r^{\alpha_{q-2}+1} J_{ \pm \mu_{q-2, n}}(\lambda r) d r \wedge \tilde{d} \phi^{(q-2)} \\
\psi_{ \pm, E, \lambda}^{(q)} & =r^{\alpha_{q}} J_{\left|\alpha_{q}\right|}(\lambda r) h^{(q)} \\
\psi_{ \pm, O, \lambda}^{(q)} & =\partial_{r}\left(r^{\alpha_{q-1}} J_{\left|\alpha_{q-1}\right|}(\lambda r)\right) d r \wedge h^{(q-1)},
\end{aligned}
$$

onde $\phi_{n}^{(q-2)}, \phi_{n}^{(q-1)}, \phi_{n}^{(q)}, h^{(q-1)}$ e $h^{(q)}$ são como no enunciado do teorema. Agora fazendo uma verificação simples nas soluções vemos que as soluções $(+)$ são quadrado integráveis e satisfazem as condições de contorno em $r=0$ (para tais condições ver [5]), definidas no domínio de $\Delta^{(q)}$. No caso (-), todas elas ou não são quadrado integráveis ou não satisfazem a condição de contorno em $r=0$. O resultado segue. Um prova completa pode ser encontrada em [34] seção 5 para o caso das harmônicas.

\subsection{Ferramentas analíticas}

Nesta seção apresentaremos todas as ferramentas necessárias para avaliar as funções zetas que aparecem no cálculo da Torção Analítica. Para isso teremos como referência os artigos [42], 43], 44] e [46]. Apresentamos aqui um versão simplificada do resultado principal destes trabalhos (ver em particular a formulação geral no teorema 3.9 de [46] ou o Lema da Decomposição Espectral de [44]), que é o suficiente para os nossos propósitos.

Primeiro algumas definições básicas. A função zeta de Riemann, denota da por $\zeta_{R}(s)$, é a função

$$
\zeta_{R}(s)=\sum_{n=1}^{\infty} \frac{1}{n^{s}},
$$

que é definida para todo $s$ com parte real maior que 1 . Sabemos que $\zeta_{R}(s)$ possui uma única continuação analítica em todo o plano complexo, excluindo o ponto $s=1$ que corresponde ao

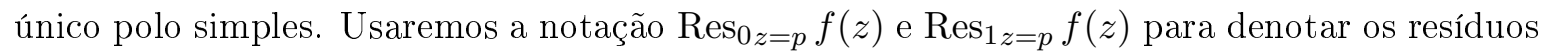
de grau zero e um, respectivamente, no ponto $z=p$ da função $f(z)$. Por exemplo, no caso da função zeta de Riemann temos que

$$
\operatorname{Res}_{s=1} \zeta_{R}(s)=\gamma, \quad \operatorname{Res}_{s=1} \zeta_{R}(s)=1,
$$

onde $\gamma \simeq 0,577216$ é a constante de Euler. 
Seja $S=\left\{a_{n}\right\}_{n=1}^{\infty}$ uma seqüência de números reais (os resultados valem em geral para números complexos) ordenados $\left|a_{0}\right| \leq\left|a_{1}\right| \leq \ldots$, com um único ponto de acumulação no infinito. O limite superior

$$
s_{0}=\limsup _{n \rightarrow \infty} \frac{\log n}{\log \left|a_{n}\right|},
$$

é chamado o expoente de convergência de $S$ e é também denotado por e $(S)$. Este é um número real positivo ou infinito. Estamos interessados apenas em casos em que e $(S)=s_{0}<\infty$. Neste caso, existe um menor número inteiro $p$ tal que a série $\sum_{n=1}^{\infty} a_{n}^{-p-1}$ converge absolutamente. Assumimos que $s_{0}-1<p \leq s_{0}$ e chamaremos o inteiro $p$ de genus da seqüência $S$, e escreveremos $p=\mathrm{g}(S)$. Definimos a função zeta associada a $S$ pela série uniformemente convergente

$$
\zeta(s, S)=\sum_{n=1}^{\infty} a_{n}^{-s},
$$

quando $\operatorname{Re}(s)>\mathrm{e}(S)$, e quando isto não ocorre, por sua continuação analítica. Chamaremos o subconjunto aberto do plano complexo $\rho(S)=\mathbb{C}-S$ de conjunto resolvente de $S$. Para todo $\lambda \in \rho(S)$, definimos a função Gamma associada a $S$ por meio do produto,

$$
\frac{1}{\Gamma(-\lambda, S)}=\prod_{n=1}^{\infty}\left(1+\frac{-\lambda}{a_{n}}\right) \mathrm{e}^{\sum_{j=1}^{\mathrm{g}(S)} \frac{(-1)^{j}}{j} \frac{(-\lambda)^{j}}{a_{n}^{j}}} .
$$

Quando necessário, no sentido de definir o ramo meromorfo de uma função analítica, o domínio para $\lambda$ será o subconjunto aberto do plano complexo, $\mathbb{C}-[0, \infty)$. Usaremos a notação $\Sigma_{\theta, c}=\left\{z \in \mathbb{C}|| \arg (z-c) \mid \leq \frac{\theta}{2}\right\}, \operatorname{com} c \geq \delta>0,0<\theta<\pi$, e ainda, $D_{\theta, c}=\mathbb{C}-\Sigma_{\theta, c}$, para o domínio complementar (aberto) e $\Lambda_{\theta, c}=\partial \Sigma_{\theta, c}=\left\{z \in \mathbb{C}|| \arg (z-c) \mid=\frac{\theta}{2}\right\}$ para o bordo, que é orientado no sentido anti-horário. Com esta notação, definimos uma subclasse particular de seqüências.

Definição 12. Seja $S$ como anteriormente, assuma que $\mathrm{e}(S)<\infty$ e que exista $c>0$ e $0<\theta<\pi$, tal que $S$ está contida no interior do setor $\Sigma_{\theta, c}$. Mais ainda, assuma que o logaritmo da função Gamma associada possua uma expansão assintótica uniforme para $\lambda$ suficientemente grande, $\lambda \in D_{\theta, c}(S)=\mathbb{C}-\Sigma_{\theta, c}$, da seguinte forma

$$
\log \Gamma(-\lambda, S) \sim \sum_{j=0}^{\infty} a_{\alpha_{j}, 0}(-\lambda)^{\alpha_{j}}+\sum_{k=0}^{\mathrm{g}(S)} a_{k, 1}(-\lambda)^{k} \log (-\lambda),
$$

onde $\left\{\alpha_{j}\right\}$ é uma seqüência decrescente de números reais. Então, diremos que $S$ é uma seqüência totalmente regular do tipo espectral com ordem infinita e chamaremos o conjunto aberto $D_{\theta, c}(S)$ de o dominio assintótico de $S$.

Seja $S=\left\{\lambda_{n, k}\right\}_{n, k=1}^{\infty}$ uma seqüência dupla (ver [12]) de números reais não nulos (os resultados valem em geral para números complexos) com um único ponto de acumulação no infinito, expoente finito $s_{0}=\mathrm{e}(S)$ e genus $p=\mathrm{g}(S)$. Assuma, se necessário, que os elementos 
de $S$ estejam ordenados como segue $0<\left|\lambda_{1,1}\right| \leq\left|\lambda_{1,2}\right| \leq\left|\lambda_{2,1}\right| \leq \ldots$ Usaremos a notação $S_{n}$ $\left(S_{k}\right)$ para denotar a seqüência simples com índice fixado $n(k)$. Chamaremos os expoentes de $S_{n}$ e $S_{k}$ de expoentes relativos de $S$ e usaremos a notação $\left(s_{0}=\mathrm{e}(S), s_{1}=\mathrm{e}\left(S_{k}\right), s_{2}=\mathrm{e}\left(S_{n}\right)\right)$. Definiremos o genus relativo da mesma maneira.

Definição 13. Seja $S=\left\{\lambda_{n, k}\right\}_{n, k=1}^{\infty}$ uma seqüência dupla com expoentes finitos $\left(s_{0}, s_{1}, s_{2}\right)$, genus $\left(p_{0}, p_{1}, p_{2}\right)$, e setor espectral positivo $\Sigma_{\theta_{0}, c_{0}}$. Seja $U=\left\{u_{n}\right\}_{n=1}^{\infty}$ uma seqüência totalmente regular do tipo espectral com ordem infinita com expoente $r_{0}$, genus $q$, dominio $D_{\phi, d}$. Diremos que $S$ é espectralmente decomposta sobre $U$ com potência $\kappa$, comprimento $\ell$ e domínio assintótico $D_{\theta, c}$, com $c=\min \left(c_{0}, d, c^{\prime}\right), \theta=\max \left(\theta_{0}, \phi, \theta^{\prime}\right)$, se existem números reais positivos $\kappa, \ell$ (inteiro), $c^{\prime}$, e $\theta^{\prime}$, com $0<\theta^{\prime}<\pi$, tal que:

1. A seqüência $u_{n}^{-\kappa} S_{n}=\left\{\frac{\lambda_{n, k}}{u_{n}^{\kappa}}\right\}_{k=1}^{\infty}$ possui setor espectral $\Sigma_{\theta^{\prime}, c^{\prime}}$ e é uma seqüência totalmente regular do tipo espectral com ordem infinita para cada $n$;

2. O logaritmo da função $\Gamma$ associada a $S_{n} / u_{n}^{\kappa}$ possui uma expansão assintótica, para $n$ grande, uniformemente em $\lambda$, para $\lambda$ em $D_{\theta, c}$, da seguinte forma

$$
\log \Gamma\left(-\lambda, u_{n}^{-\kappa} S_{n}\right)=\sum_{h=0}^{\ell} \phi_{\sigma_{h}}(\lambda) u_{n}^{-\sigma_{h}}+\sum_{l=0}^{L} P_{\rho_{l}}(\lambda) u_{n}^{-\rho_{l}} \log u_{n}+o\left(u_{n}^{-r_{0}}\right),
$$

onde $\sigma_{h}$ e $\rho_{l}$ são números reais com $\sigma_{0}<\cdots<\sigma_{\ell}, \rho_{0}<\cdots<\rho_{L}$, os $P_{\rho_{l}}(\lambda)$ são polinômios em $\lambda$ satisfazendo a condição $P_{\rho_{l}}(0)=0$, $\ell$ e L são os maiores inteiros tais que $\sigma_{\ell} \leq r_{0}$ e $\rho_{L} \leq r_{0}$.

Quando uma seqüência dupla $S$ é espectralmente decomposta sobre uma seqüência simples $U$, o teorema 3.9 de [46] fornece uma fórmula para a derivada da função zeta associada a $S$ no zero. No sentido de entender tal fórmula, precisamos introduzir algumas quantidades. Primeiro, definimos as funções

$$
\Phi_{\sigma_{h}}(s)=\int_{0}^{\infty} t^{s-1} \frac{1}{2 \pi i} \int_{\Lambda_{\theta, c}} \frac{\mathrm{e}^{-\lambda t}}{-\lambda} \phi_{\sigma_{h}}(\lambda) d \lambda d t .
$$

Segundo, pelo lema 3.3 de [46], para todo $n$, temos as expansões:

$$
\begin{aligned}
\log \Gamma\left(-\lambda, S_{n} / u_{n}^{\kappa}\right) & \sim \sum_{j=0}^{\infty} a_{\alpha_{j}, 0, n}(-\lambda)^{\alpha_{j}}+\sum_{k=0}^{p_{2}} a_{k, 1, n}(-\lambda)^{k} \log (-\lambda), \\
\phi_{\sigma_{h}}(\lambda) & \sim \sum_{j=0}^{\infty} b_{\sigma_{h}, \alpha_{j}, 0}(-\lambda)^{\alpha_{j}}+\sum_{k=0}^{p_{2}} b_{\sigma_{h}, k, 1}(-\lambda)^{k} \log (-\lambda),
\end{aligned}
$$

para $\lambda$ grande em $D_{\theta, c}$. Definimos (ver lema 3.5 de [46])

$$
\begin{aligned}
& A_{0,0}(s)=\sum_{n=1}^{\infty}\left(a_{0,0, n}-\sum_{h=0}^{\ell} b_{\sigma_{h}, 0,0} u_{n}^{-\sigma_{h}}\right) u_{n}^{-\kappa s}, \\
& A_{j, 1}(s)=\sum_{n=1}^{\infty}\left(a_{j, 1, n}-\sum_{h=0}^{\ell} b_{\sigma_{h}, j, 1} u_{n}^{-\sigma_{h}}\right) u_{n}^{-\kappa s}, \quad 0 \leq j \leq p_{2} .
\end{aligned}
$$


Agora podemos apresentar a derivada no zero da função zeta dupla. Apresentamos uma versão modificada do teorema 3.9 de [46], mais simples para os nossos propósitos aqui, que é fundamentada nos seguintes fatos. O ponto chave na prova do teorema 3.9 de [46] é a decomposição dada no lema 3.5 deste artigo sobre a soma

$$
\mathcal{T}(s, \lambda, S, U)=\sum_{n=1}^{\infty} u_{n}^{-\kappa s} \log \Gamma\left(-\lambda, u_{n}^{-\kappa} S_{n}\right)
$$

em duas partes: a parte regular $\mathcal{P}(s, \lambda, S, U)$ e a parte singular remanescente. A parte regular é obtida subtraindo de $\mathcal{T}$ alguns termos construídos a partir da expansão do logaritmo da função Gamma dada na equação (1.18), a saber

$$
\mathcal{P}(s, \lambda, S, u)=\mathcal{T}(s, \lambda, S, U)-\sum_{h=0}^{\ell} \phi_{\sigma_{h}}(\lambda) u_{n}^{-\sigma_{h}}-\sum_{l=0}^{L} P_{\rho_{l}}(\lambda) u_{n}^{-\rho_{l}} \log u_{n} .
$$

Assuma que subtraímos apenas os termos tais que a função zeta $\zeta(s, U)$ possui um polo em $s=\sigma_{h}$ ou em $s=\rho_{l}$. Seja $\hat{\mathcal{P}}(s, \lambda, S, U)$ a função resultante. Então o mesmo argumento que foi usando na seção 3 de [46] para provar o teorema 3.9 se aplica. Assim, obtemos fórmulas similares para os valores dos resíduos e para partes finitas da função zeta $\zeta(s, S)$ e de sua derivada no zero, com apenas duas diferenças, a saber, em toda a soma, todos os termos com índices $\sigma_{h}$ tais que $s=\sigma_{h}$ não é um polo de $\zeta(s, U)$ devem ser omitidos. Além disso devemos substituir os termos $A_{0,0}(0)$ e $A_{0,1}^{\prime}(0)$ pelas partes finitas da extensão analítica de $A_{0,0}(s)$ e $A_{0,1}^{\prime}(s)$, respectivamente. A primeira modificação é uma conseqüência da substituição da função $\mathcal{P}$ pela função $\hat{\mathcal{P}}$. A segunda modificação segue pela mesma razão notando que as funções $A_{\alpha_{j}, k}(s)$, definidas no lema 3.5 de [46], não são mais regulares em $s=0$. No entanto, ambas admitem um extensão meromorfa regular em $s=0$, usando a extensão da função zeta $\zeta(s, U)$ e a expansão dos coeficientes $a_{\alpha_{j}, k, n}$ para $n$ suficientemente grande. Então temos o resultado a seguir:

Teorema 4. As fórmulas do teorema 3.9 de [46] valem se todas as quantidade com índices $\sigma_{h}$ tal que a função zeta $\zeta(s, U)$ não possua polo em $s=\sigma_{h}$ são omitidas. Neste caso, o resultado deve ser entendido por meio da extensão analítica da função zeta $\zeta(s, U)$.

Assumindo uma estrutura simplificada dos polos para a função $\zeta(s, U)$, suficiente para a presente análise, afirmamos o resultado principal desta seção.

Teorema 5. Seja $S$ uma seqüencia espectralmente decomposta sobre U como na definição 13. Assuma que a função $\Phi_{\sigma_{h}}(s)$ possui no máximo polos simples em $s=0$. Então, $\zeta(s, S)$ é 
regular em $s=0$, $e$

$$
\begin{aligned}
\zeta(0, S)= & -A_{0,1}(0)+\frac{1}{\kappa} \sum_{h=0}^{\ell} \operatorname{Res}_{s=0} \Phi_{\sigma_{h}}(s) \operatorname{Res}_{s=\sigma_{h}} \zeta(s, U), \\
\zeta^{\prime}(0, S)= & -A_{0,0}(0)-A_{0,1}^{\prime}(0)+\frac{\gamma}{\kappa} \sum_{h=0}^{\ell} \operatorname{Res}_{s=0} \Phi_{\sigma_{h}}(s) \operatorname{Res}_{s=\sigma_{h}} \zeta(s, U) \\
& +\frac{1}{\kappa} \sum_{h=0}^{\ell} \operatorname{Res}_{s=0} \Phi_{\sigma_{h}}(s) \operatorname{Res}_{s=\sigma_{h}} \zeta(s, U)+\sum_{h=0}^{\ell} \operatorname{Res}_{s=0}^{\prime} \Phi_{\sigma_{h}}(s) \operatorname{Res}_{s=\sigma_{h}} \zeta(s, U),
\end{aligned}
$$

onde a notação $\sum^{\prime}$ significa que apenas os termos tais que $\zeta(s, U)$ possui um polo em $s=\sigma_{h}$, aparecem na soma.

Este resultado deve ser comparado com o Lema da Decomposição Espectral de [44] e a Proposição 1 de [45].

Corolário 1. Sejam $S_{(j)}=\left\{\lambda_{(j), n, k}\right\}_{n, k=1}^{\infty}, j=1,2$, duas seqüências duplas que satisfazem tudo o que é pedido na definiçẫo 13 da decomposiçẫo sobre uma seqüência em comum $U$, com os mesmos parâmetros $\kappa, \ell$, etc., exceto que o polinômio $P_{(j), \rho}(\lambda)$ que aparece na condição (2) é não nulo em $\lambda=0$. Assuma que a diferença destes polinômios satisfaçam esta condição, ou seja, $P_{(1), \rho}(0)-P_{(2), \rho}(0)=0$. Então, a diferença das funções zeta $\zeta\left(s, S_{(1)}\right)-\zeta\left(s, S_{(2)}\right)$ é regular em $s=0$ e satisfaz as fórmulas dadas no teorema 5 .

Concluímos esta seção lembrando alguns resultados de funções zetas de algumas seqüências simples que serão necessárias a seguir e uma fórmula para a integral de contorno que aparece no texto. Usaremos a formulação de [41]. Para números reais positivos $l$ e $q$, defina a função zeta quadrática de Bessel não homogênea por

$$
z(s, \nu, q, l)=\sum_{k=1}^{\infty}\left(\frac{j_{\nu, k}^{2}}{l^{2}}+q^{2}\right)^{-s},
$$

para $\operatorname{Re}(s)>\frac{1}{2}$. Então $z(s, \nu, q, l)$ estende analiticamente a uma função meromorfa no plano complexo com polos simples em $s=\frac{1}{2},-\frac{1}{2},-\frac{3}{2}, \ldots,-\frac{2 k-1}{2}$ para todo $k \in \mathbb{N}$. O ponto $s=0$ é um ponto regular e

$$
\begin{aligned}
z(0, \nu, q, l) & =-\frac{1}{2}\left(\nu+\frac{1}{2}\right), \\
z^{\prime}(0, \nu, q, l) & =-\log \sqrt{2 \pi l} \frac{I_{\nu}(l q)}{q^{\nu}} .
\end{aligned}
$$

Em particular, considerando o limite para $q \rightarrow 0$ temos,

$$
z^{\prime}(0, \nu, 0, l)=-\log \frac{\sqrt{\pi} l^{\nu+\frac{1}{2}}}{2^{\nu-\frac{1}{2}} \Gamma(\nu+1)} .
$$

Para terminar, seja $\Lambda_{\theta, c}=\{\lambda \in \mathbb{C}|| \arg (\lambda-c) \mid=\theta\}, 0<\theta<\pi, 0<c<1$, a real, então

$$
\int_{0}^{\infty} t^{s-1} \frac{1}{2 \pi \mathrm{i}} \int_{\Lambda_{\theta, c}} \frac{\mathrm{e}^{-\lambda t}}{-\lambda} \frac{1}{(1-\lambda)^{a}} d \lambda d t=\frac{\Gamma(s+a)}{\Gamma(a) s} .
$$

A prova da equação (1.23) pode ser encontrada em [42] seção 4.2. 
1. Preliminares 


\section{Capítulo 2}

\section{A Torção de Reidemeister e a Torção Analítica}

Este capítulo é fundamentado em [4], [27] e [36]. Apresentamos as definições da Torção Analítica e a Torção de Reidemeister, resultados para a comparação entre as duas torções e ainda a motivação encontrada por D.B. Ray e I.M. Singer para a definição da Torção Analítica.

\subsection{Torção de Reidemeister}

Se $V$ é um espaço vetorial real de dimensão finita, $v=\left\{v_{1}, \ldots, v_{k}\right\}$ e $w=\left\{w_{1}, \ldots, w_{k}\right\}$ são duas bases para $V$, seja $(w / v)$ a matriz de mudança de base de $v$ para $w$, ou seja, $w_{i}=\sum_{j=1}^{k}(w / v)_{i j} v_{j}$.

Seja

$$
C: \quad C_{m} \stackrel{\partial_{m}}{\longrightarrow} C_{m-1} \stackrel{\partial_{m-1}}{\longrightarrow} \cdots \stackrel{\partial_{2}}{\longrightarrow} C_{1} \stackrel{\partial_{1}}{\longrightarrow} C_{0},
$$

um complexo de cadeia (de dimensão finita) de espaços vetoriais reais. Denote por $Z_{q}=$ ker $\partial_{q}$, $B_{q}=\operatorname{Im} \partial_{q+1}$, e $H_{q}(C)=Z_{q} / B_{q}$ como usual. Fixe uma base preferida $\mathbf{c}_{q}$ para $C_{q}$, e uma base $\mathbf{h}_{q}$ para $H_{q}(C)$. Escolha uma base $\mathbf{b}_{q}$ para $B_{q}$ e seja $\tilde{\mathbf{b}}_{q-1}$ um conjunto independente de elementos em $C_{q}$ tal que $\partial\left(\tilde{\mathbf{b}}_{q-1}\right)=\mathbf{b}_{q-1}$. Então o conjunto de elementos $\left\{\tilde{\mathbf{b}}_{q-1}, \mathbf{h}_{q}, \mathbf{b}_{q}\right\}$ é uma base para $C_{q}$. Nessa situação, definimos

Definição 14 (Torção de Reidemeister). A Torção de Reidemeister do complexo $C$ com respeito a base $\mathbf{h}=\left\{\mathbf{h}_{q}\right\}$ é o número real definido por

$$
\log \tau(C ; \mathbf{h})=\sum_{q=0}^{m}(-1)^{q} \log \left|\operatorname{det}\left(\tilde{\mathbf{b}}_{q-1}, \mathbf{h}_{q}, \mathbf{b}_{q} / \mathbf{c}_{q}\right)\right|
$$

Note que $\tau(C ; \mathbf{h})$ não depende da escolha das bases $\mathrm{b}_{q}$ de $B_{q}$, de fato, se $\mathrm{b}_{q}^{\prime}$ é uma outra 
escolha então

$$
\left(\tilde{\mathbf{b}}_{q-1}^{\prime}, \mathbf{h}_{q}, \mathbf{b}_{q}^{\prime} / \mathbf{c}_{q}\right)=\left(\tilde{\mathbf{b}}_{q-1}^{\prime} / \tilde{\mathbf{b}}_{q-1}\right)\left(\tilde{\mathbf{b}}_{q-1}, \mathbf{h}_{q}, \mathbf{b}_{q} / \mathbf{c}_{q}\right)\left(\mathbf{b}_{q}^{\prime} / \mathbf{b}_{q}\right)
$$

Agora usando essa igualdade na definição, os fatores $\left(\tilde{\mathbf{b}}_{q-1}^{\prime} / \tilde{\mathbf{b}}_{q-1}\right)$ e $\left(\mathbf{b}^{\prime}{ }_{q} / \mathbf{b}_{q}\right)$ desaparecem na fórmula de $\tau(C ; \mathbf{h})$. Seja $(K, L)$ um par de complexos celulares de dimensão finita $m$, e $(\tilde{K}, \tilde{L})$ seu recobrimento universal celular, identificamos o grupo fundamental de $K, \pi_{1}=\pi_{1}(K)$, com o grupo de transformações de recobrimento de $\tilde{K}$. Seja $C((\tilde{K}, \tilde{L}) ; \mathbb{R})$ o complexo de cadeia de $(\tilde{K}, \tilde{L})$ com coeficientes em $\mathbb{R}$. A ação do grupo de transformações de recobrimento transforma cada $C_{q}((\tilde{K}, \tilde{L}) ; \mathbb{R})$ em um módulo sobre o anel de grupo $\mathbb{R} \pi_{1}(K)$, com a base preferida sendo as células de $K-L$. Temos então um complexo de módulos finitamente gerados sobre $\mathbb{R} \pi_{1}(K)$ que, seguindo a notação padrão, denotaremos por $C\left((\tilde{K}, \tilde{L}) ; \mathbb{R} \pi_{1}(K)\right)$. Considere $\rho$ uma representação de $\pi_{1}(K)$ em $O(k, \mathbb{R})$, o grupo das matrizes ortogonais $k \times k$. A representação $\rho$ faz $\mathbb{R}^{k}$ um $\mathbb{R} \pi_{1}$-módulo a direita. Defina o complexo de cadeia $C(K, L ; \rho)$ por

$$
C_{q}(K, L ; \rho)=\mathbb{R}^{k} \otimes_{\mathbb{R} \pi_{1}} C_{q}\left((\tilde{K}, \tilde{L}) ; \mathbb{R} \pi_{1}(K)\right) .
$$

$C_{q}(K, L, \rho)$ é um espaço vetorial real. Dessa forma podemos escolher uma base preferida $e_{j} \otimes \mathbf{c}_{q}$ para $C_{q}(K, L ; \rho)$ com sendo $\mathbf{c}_{q}$ base preferida de $C_{q}\left((\tilde{K}, \tilde{L}) ; \mathbb{R} \pi_{1}(K)\right)$ e $e_{j}$ elementos de uma base ortogonal de $\mathbb{R}^{k}$. Definimos,

Definição 15 (Torção de Reidemeister de um complexo celular). A Torção de Reidemeister de $(K, L)$ para a base $\mathbf{h}=\left\{\mathbf{h}_{q}\right\}$ é

$$
\tau(K, L ; \mathbf{h})=\tau(C(K, L ; \rho) ; \mathbf{h}) .
$$

Observamos que a base preferida de $C(K, L ; \rho)$ depende de uma imersão arbitrária de $(K, L)$ em $(\tilde{K}, \tilde{L})$, mas uma escolha diferente da imersão produz uma nova base relacionada com a primeira por uma matriz ortogonal e assim a Torção de Reidemeister é independente da imersão. Da mesma forma, não depende da escolha da base ortonormal para $\mathbb{R}^{k}$. Notamos ainda que se $(K, L)$ é uma decomposição celular de um espaço $(X, A)$ e $C(K, L ; \rho)$ é definido como acima, a Torção de Reidemeister de $(X, A)$ está definida e denotaremos por $\tau(X, A, \mathbf{h})$. No caso em que $C(K, L ; \rho)$ é acíclico, foi provado em [30] que $\tau(X, A)$ é um invariante combinatorial, logo não depende da decomposição celular.

Iremos trabalhar com a Torção de Reidemeister no seguinte sentido. Considere $W$ uma variedade Riemanniana, compacta, $C^{\infty}$, orientável, de dimensão $m$ e com bordo $\partial W$ (possivelmente vazio), e uma métrica Riemanniana $g$. Então, todas as afirmações anteriores são satisfeitas e podemos definir a Torção de Reidemeister absoluta $\tau((W, \emptyset) ; \mathbf{h}, \rho)$ e a Torção de Reidemeister relativa $\tau((W, \partial W) ; \mathbf{h}, \rho)$, para cada representação $\rho$ do grupo fundamental, e para cada base graduada fixada $\mathbf{h}$ para a homologia de $(W, \emptyset)$, e para a homologia relativa de $(W, \partial W)$, respectivamente. Neste contexto, Ray e Singer [36] sugeriram um objeto invariante geométrico, fixando uma base apropriada $\mathbf{h}$ usando a estrutura geométrica de $W$, como segue. 
Antes de começarmos a definir a base $\mathbf{h}$, apresentamos a definição de um fibrado vetorial associado a uma representação. Sabemos que o recobrimento universal de $\tilde{K}$ de $K$ é um fibrado principal, seja $\rho: \pi(K) \rightarrow O(k, \mathbb{R})$ uma representação, então

Definição 16. O fibrado vetorial associado a representação $\rho$ é o espaço quociente

$$
E_{\rho}=\tilde{K} \times{ }_{\rho} \mathbb{R}^{k}
$$

de $\tilde{K} \times \mathbb{R}^{k}$ pela relação $(u, v) \sim\left(u g, \rho(g)^{-1} v\right)$, onde $u \in \tilde{K}, v \in \mathbb{R}$ e $g \in \pi_{1}(W)$.

Sejam $E_{\rho} \rightarrow W$ o fibrado vetorial associado a representação $\rho: \pi_{1}(W) \rightarrow O(k, \mathbb{R})$ e $\Omega\left(W, E_{\rho}\right)$ o espaço graduado das formas lineares suaves em $W$ com com valores em $E_{\rho}$. Uma base para $\Omega^{q}\left(W, E_{\rho}\right)$ é da forma $\left\{C^{\infty}(W) \otimes d x_{I} \otimes_{\rho} e_{i}\right\}$. A diferencial exterior em $W$ define a diferencial exterior de $\Omega^{q}\left(W, E_{\rho}\right), d: \Omega^{q}\left(W, E_{\rho}\right) \rightarrow \Omega^{q+1}\left(W, E_{\rho}\right)$. A métrica $g$ define um operador de Hodge em $W$, ou seja, em $\Omega^{q}\left(W, E_{\rho}\right), *: \Omega^{q}\left(W, E_{\rho}\right) \rightarrow \Omega^{m-q}\left(W, E_{\rho}\right)$. Com isso podemos definir o adjunto formal de $d, d^{\dagger}=(-1)^{m q+m+1} * d *$, onde $d^{\dagger}: \Omega^{q}\left(W, E_{\rho}\right) \rightarrow$ $\Omega^{q-1}\left(W, E_{\rho}\right)$, e o Laplaciano $\Delta=\left(d+d^{\dagger}\right)^{2}, \Delta^{(q)}: \Omega^{q}\left(W, E_{\rho}\right) \rightarrow \Omega^{q}\left(W, E_{\rho}\right)$. Usando o produto interno em $E_{\rho}$, definimos um produto interno em $\Omega^{q}\left(W, E_{\rho}\right)$, como segue,

$$
\omega=\sum_{I, j} \omega_{I, j}(x) d x_{I} \otimes e_{j}, \quad \quad \eta=\sum_{I^{\prime}, j^{\prime}} \eta_{I^{\prime}, j^{\prime}}(x) d x_{I^{\prime}} \otimes e_{j^{\prime}},
$$

então

$$
(\omega, \eta)=\sum_{I, I^{\prime}, j, j^{\prime}} \int_{W} \omega_{I, j}(x) \omega_{I^{\prime}, j^{\prime}}(x) d x_{I} \wedge * d x_{I^{\prime}}\left(e_{j}, e_{j^{\prime}}\right),
$$

onde $(v, w)=v^{T} w$ é o produto interno em $\mathbb{R}^{k}$ e $\wedge$ é o produto exterior em $\Omega(W)$.

Iremos trabalhar com variedades com bordo, logo é necessário introduzir ferramentas específicas. Para ser mais preciso, se $W$ possui um bordo $\partial W$, então existe uma cisão natural de $\Omega(W)$ como a soma direta dos fibrados vetoriais $N^{*} W \oplus \Omega(\partial W)$, onde $N^{*} W$ é o dual do fibrado normal no bordo. Localmente, seja $\partial_{r}$ denotando o vetor unitário normal apontando para fora da variedade e $d r$ a 1-forma correspondente. Próximo do bordo temos um colar $\mathcal{C}(\partial W)=(-\epsilon, 0] \times \partial W$ e, se $y$ é um sistema de coordenadas local no bordo, então $(r, y)$ é um sistema de coordenadas local em $\mathcal{C}(\partial W)$. Nesse sistema de coordenadas, o tensor métrico é da forma

$$
g=d r \otimes d r+\tilde{g}(r)
$$

onde $\tilde{g}(r)$ é uma família de métricas em $\partial W$ tal que $\tilde{g}(0)=i^{*} g$ e $i: \partial W \hookrightarrow W$ é a inclusão.

Dada uma forma $\omega \in \Omega(W)$, próxima ao bordo esta se decompõe em

$$
\omega=\omega_{\tan }+\omega_{\text {norm }}
$$

onde $\omega_{\text {norm }}$ é a projeção ortogonal do subespaço gerado por $d r$, e $\omega_{\tan } \in \Omega(\partial W)$, então

$$
\omega=\omega_{1}+d r \wedge \omega_{2}
$$


onde $\omega_{1}, \omega_{2} \in C^{\infty}(W) \otimes \Omega(\partial W)$ tal que

$$
* \omega_{2}=-d r \wedge * \omega
$$

Definimos a condição de contorno $B_{\text {rel }}$ e $B_{\text {abs }}$ por

$$
B_{\text {rel }}(\omega)=\left.\omega_{\tan }\right|_{\partial W}=\left.\omega_{1}\right|_{\partial W}=0 \text { e } B_{\text {abs }}(\omega)=\left.\omega_{\text {norm }}\right|_{\partial W}=\left.\omega_{2}\right|_{\partial W}=0,
$$

respectivamente.

Lema 3. Seja $\omega \in \Omega^{q}(W)$ então $B_{\text {abs }}(\omega)=0 \Leftrightarrow B_{\text {rel }}(* \omega)=0, B_{\text {rel }}(\omega)=0 \Rightarrow B_{\text {rel }}(d \omega)=0$ e $B_{\text {abs }}(\omega)=0 \Rightarrow B_{\text {abs }}\left(d^{\dagger} \omega\right)=0$. ([20], Lema 2.7.1).

Demonstração. Dada uma forma $\omega \in \Omega^{q}(W)$, proxima ao bordo $\omega$ se escreve como em (2.5). Logo

$$
*(\omega)=*\left(\omega_{1}+d r \wedge \omega_{2}\right)=d r \wedge \tilde{*} \omega_{1}+\tilde{*} \omega_{2},
$$

onde $\tilde{*}$ é o operado estrela de hodge do bordo. Agora $B_{\text {abs }}(\omega)=\left.\omega_{2}\right|_{\partial W}=0$ se, e somente se, $B_{\text {rel }}(* \omega)=\left.\tilde{*} \omega_{2}\right|_{\partial W}=0$. Se $B_{\text {rel }}(\omega)=\left.\omega_{1}\right|_{\partial W}=0$ então

$$
d \omega=\tilde{d} \omega_{1}+d r \wedge\left(-\partial_{r} \omega_{1}+\tilde{d} \omega_{2}\right),
$$

onde $\tilde{d}$ é a derivada exterior do $\partial W$. Assim $B_{\text {rel }}(d \omega)=\left.\tilde{d} \omega_{1}\right|_{\partial W}=0$. A última afirmação segue pela dualidade de Hodge e da primeira equivalência.

Sejam $B \in\left\{B_{\text {abs }}, B_{\text {rel }}\right\}$ e $\mathcal{B}(\omega)=B(\omega) \oplus B\left(\left(d+d^{\dagger}\right)(\omega)\right)$. Então o operador $\Delta=\left(d+d^{\dagger}\right)^{2}$ com condições de contorno no bordo $\mathcal{B}(\omega)=0$ é auto-adjunto ([20], Lema 2.7.2).

Definição 17. As condições de contorno absoluta são

$$
\mathcal{B}_{\mathrm{abs}}(\omega)=0 \quad \text { se e somente se }\left\{\begin{array}{l}
\left.\omega_{\text {norm }}\right|_{\partial W}=0, \\
\left.(d \omega)_{\text {norm }}\right|_{\partial W}=0,
\end{array}\right.
$$

e as condições de contorno relativa são

$$
\mathcal{B}_{\text {rel }}(\omega)=0 \quad \text { se e somente se }\left\{\begin{array}{l}
\left.\omega_{\tan }\right|_{\partial W}=0, \\
\left.\left(d^{\dagger} \omega\right)_{\tan }\right|_{\partial W}=0 .
\end{array}\right.
$$

\section{Definição 18.}

$$
\begin{gathered}
\mathcal{H}_{\mathrm{abs}}^{q}\left(W, E_{\rho}\right)=\left\{\omega \in \Omega^{q}\left(W, E_{\rho}\right) \mid \Delta^{(q)} \omega=0, B_{\mathrm{abs}}(\omega)=0\right\}, \\
\mathcal{H}_{\mathrm{rel}}^{q}\left(W, E_{\rho}\right)=\left\{\omega \in \Omega^{q}\left(W, E_{\rho}\right) \mid \Delta^{(q)} \omega=0, B_{\text {rel }}(\omega)=0\right\},
\end{gathered}
$$

são os espaços das formas harmônicas com condiçôes de contorno absoluta e relativa (respectivamente) no bordo. 
Observamos que a condição $\Delta^{(q)} \omega=0, B_{\text {abs }}(\omega)=0$ é equivalente a $\Delta^{(q)} \omega=0, \mathcal{B}_{\text {abs }}(\omega)=0$ (respectivamente para o caso relativo, ver [20]). Ainda, valem as decomposições de Hodge em cada caso:

Teorema 6 (Decomposição de Hodge). Se W é uma variedade Riemanniana com bordo como acima, temos

$$
\begin{aligned}
\Omega_{\mathrm{abs}}^{q}\left(W, E_{\rho}\right) & =\mathcal{H}_{\mathrm{abs}}^{q}\left(W, E_{\rho}\right) \oplus d \Omega_{\mathrm{abs}}^{q-1}\left(W, E_{\rho}\right) \oplus d^{\dagger} \Omega_{\mathrm{abs}}^{q+1}\left(W, E_{\rho}\right) \\
\Omega_{\mathrm{rel}}^{q}\left(W, E_{\rho}\right) & =\mathcal{H}_{\mathrm{rel}}^{q}\left(W, E_{\rho}\right) \oplus d \Omega_{\mathrm{rel}}^{q-1}\left(W, E_{\rho}\right) \oplus d^{\dagger} \Omega_{\mathrm{rel}}^{q+1}\left(W, E_{\rho}\right) .
\end{aligned}
$$

Para a prova e comentários ver [20] e 36].

As aplicações de de Rham que induzem um isomorfismo na cohomologia são definidas como

$$
\mathcal{A}_{\mathrm{abs}}^{q}: \mathcal{H}_{\mathrm{abs}}^{q}\left(W, E_{\rho}\right) \rightarrow C^{q}\left(W ; E_{\rho}\right), \quad \mathcal{A}_{\mathrm{rel}}^{q}: \mathcal{H}_{\mathrm{rel}}^{q}\left(W, E_{\rho}\right) \rightarrow C^{q}\left((W, \partial W) ; E_{\rho}\right),
$$

com

$$
\mathcal{A}_{\mathrm{abs}}^{q}(\omega)\left(c \otimes_{\rho} v\right)=\mathcal{A}_{\mathrm{rel}}^{q}(\omega)\left(c \otimes_{\rho} v\right)=\int_{c}(\omega, v),
$$

onde $c \otimes_{\rho} v$ pertence a $C^{q}\left(W ; E_{\rho}\right)$, no primeiro caso, e pertence a $C^{q}\left((W, \partial W) ; E_{\rho}\right)$, no segundo caso, e $c$ é identificado com a $q$-cell (simplicial ou celular) que $c$ representa.

Faremos a construção do isomorfismo de Lefschetz-Poincaré. Considere $K$ uma decomposição celular ou simplicial $\left(C^{k}, k>2\right)$ de $W$ e $L$ de $\partial W$ (no caso de condições de contorno relativa no bordo, quando consideramos condições de contorno absoluta no bordo $L=\emptyset$ ) e $\operatorname{sd} K$ a primeira subdivisão baricêntrica de $K$. Consideremos $\hat{K}$ o complexo dos blocos duais de $K$, construído da seguinte forma: Dado uma $q$-célula(simplexo) $c$ de $K$, o bloco dual $(m-q)$ $\hat{c}$ é a união das células(simplexos) de $\operatorname{sd} K$ que tem como vértice final $c$. Se considerarmos $q$-células $c$ em $K-L$, então a coleção de células $c$ é uma base para $C_{q}(K, L ; \mathbb{R})$. Portanto a bijeção $c \rightarrow \hat{c}$, induz uma bijeção entre as bases de $C_{q}(K, L ; \mathbb{R})$ e $C_{m-q}(\hat{K}-\hat{L} ; \mathbb{R})$. Temos então o isomorfismo

$$
\varphi_{q}: C_{q}(K, L ; \mathbb{R}) \rightarrow C_{m-q}(\hat{K}-\hat{L} ; \mathbb{R}), \quad \varphi_{q}: c \mapsto \hat{c}
$$

A figura 2.1 exemplifica o isomorfismo no caso em que $K$ é o 2-disco no caso absoluto.

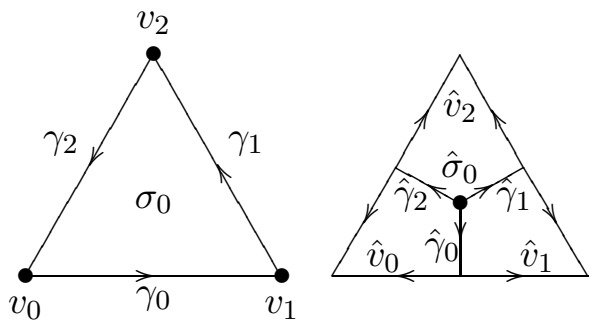

Figura 2.1: Os complexos simpliciais de $K$ e $\hat{K}$. 
Seja $c^{*}$ denotando a cocadeia dual de $c$. Então, o isomorfismo de Lefschetz-Poincaré (que induz isomorfismo da homologia sobre a cohomologia) é

$$
\begin{aligned}
& \mathcal{P}_{q}: C_{q}(K, L ; \mathbb{R}) \rightarrow C^{m-q}(\hat{K}-\hat{L} ; \mathbb{R}), \\
& \mathcal{P}_{q}: c \mapsto(\hat{c})^{*}
\end{aligned}
$$

Com estas construções estamos prontos para definir a aplicação $\mathcal{A}_{q}$ dada por

$$
\begin{array}{lll}
\mathcal{A}_{q}^{\mathrm{rel}}: \mathcal{H}_{\mathrm{rel}}^{q}\left(W, E_{\rho}\right) \rightarrow C_{q}\left((W, \partial W) ; E_{\rho}\right), & \mathcal{A}_{q}^{\mathrm{rel}}: \omega \mapsto(-1)^{(m-1) q} \mathcal{P}_{q}^{-1} \mathcal{A}_{\mathrm{abs}}^{m-q} *(\omega), \\
\mathcal{A}_{q}^{\mathrm{abs}}: \mathcal{H}_{\mathrm{abs}}^{q}\left(W, E_{\rho}\right) \rightarrow C_{q}\left(W ; E_{\rho}\right), & \mathcal{A}_{q}^{\mathrm{abs}}: \omega \mapsto(-1)^{(m-1) q} \mathcal{P}_{q}^{-1} \mathcal{A}_{\mathrm{rel}}^{m-q} *(\omega),
\end{array}
$$

ambas definidas por

$$
\mathcal{A}_{q}^{\mathrm{abs}}(\omega)=\mathcal{A}_{q}^{\mathrm{rel}}(\omega)=(-1)^{(n-1) q} \sum_{j, i}\left(\int_{\hat{c}_{q, j}}\left(* \omega, e_{i}\right)\right) c_{q, j} \otimes_{\rho} e_{i},
$$

onde a soma se dá sobre todas as $q$-células(simplexos) $c_{q, j}$ de $W$ no primeiro caso e sobre todos as $q$-células $c_{q, j}$ de $W-\partial W$ no segundo caso. E assim pela teoria de de Rham temos que $\mathcal{A}_{q}$ leva $\mathcal{H}^{q}$ sobre um conjunto de representantes das classes de homologia de $C_{q}\left(W, \partial W ; E_{\rho}\right)$ (no caso relativo) e sobre $C_{q}\left(W ; E_{\rho}\right)$ (no caso absoluto). Então, a definição da Torção de Reidemeister considerada por Ray e Singer, e que será considerada durante todo o texto daqui para frente, é definida a seguir.

Definição 19 (Torção de Reidemeister com a base de Ray e Singer). A Torção de Reidemeister com a base de Ray e Singer é definida por

$$
\tau(W, \partial W)=\tau\left(C(K, L ; \rho) ; \mathbf{a}_{\text {rel }}\right)
$$

para o par $(W, \partial W)$ (caso relativo)

$$
\tau(W)=\tau\left(C(K ; \rho) ; \mathbf{a}_{\mathrm{abs}}\right)
$$

e para $W$ (caso absoluto), onde $\mathbf{a}_{\mathrm{rel}}$ (resp. $\left.\mathbf{a}_{\mathrm{abs}}\right)$ é a imagem de uma base ortonormal para as formas harmônicas de $W$ com condiçôes de contorno relativas (resp. absolutas) no bordo por $\mathcal{A}_{q}^{\mathrm{rel}}$ (resp. $\left.\mathcal{A}_{q}^{\mathrm{abs}}\right)$, para cada $q$.

Observamos que a mudança de uma base ortonormal para outra é feita por uma matrix ortogonal, assim a torção definida acima é independente da escolha da base ortonormal. Do mesmo modo que o anterior, a Torção de Reidemeister neste caso é independente da escolha dos representantes das células de $K$ em $\tilde{K}$, ainda:

Proposição 3 (Invariância da decomposição celular). A Torção de Reidemeister $\tau(W, \partial W)$ (resp. $\tau(W)$ ) é independente da escolha da decomposição celular de $W$.

A prova desse fato pode ser encontrada em [36]. Observamos que, com a proposição [3, a Torção de Reidemeister com a base de Ray e Singer é uma função apenas da representação e da métrica associada a $W$. 


\subsection{Torção Analítica}

Seja $W$ como na seção anterior. Consideremos primeiro o caso em que $W$ não possui bordo. Com o produto interno definido na equação (2.3),$\Omega\left(W, E_{\rho}\right)$ é um espaço de Hilbert. Seja $d^{\dagger}=(-1)^{m q+m+1} * d *$ o adjunto formal de $d$. Então o Laplaciano

$$
\Delta=\left(d+d^{\dagger}\right)^{2}: \Omega\left(W, E_{\rho}\right) \rightarrow \Omega\left(W, E_{\rho}\right)
$$

é um operador simétrico, não negativo definido e possui um espectro pontual $\operatorname{Sp} \Delta$. Entendemos por espectro pontual $\operatorname{Sp} \Delta$, se para todo $\lambda \in \operatorname{Sp} \Delta, \lambda$ é autovalor de $\Delta$. Seja $\Delta^{(q)}$ a restrição de $\Delta$ ao espaço $\Omega^{q}\left(W, E_{\rho}\right)$.

Definição 20. A função zeta de $\Delta^{(q)}$ é a serie

$$
\zeta\left(s, \Delta^{(q)}\right)=\sum_{\lambda \in \mathrm{Sp}_{+} \Delta^{(q)}} \lambda^{-s},
$$

para $\operatorname{Re}(s)>\frac{m}{2}$, e onde $\mathrm{Sp}_{+}$denota a parte positiva do espectro de $\Delta^{(q)}$.

A série anterior converge uniformemente para $\operatorname{Re}(s)>\frac{m}{2}$ e estende a uma função meromorfa analítica em $s=0$. Seguindo [36] definimos

Definição 21 (Torção Analítica). A Torção Analítica de W com uma métrica g é definida por

$$
\log T(W, g)=\frac{1}{2} \sum_{q=1}^{m}(-1)^{q} q \zeta^{\prime}\left(0, \Delta^{(q)}\right) .
$$

Omitiremos a métrica $g$ quando não houver risco de confusão. Se $W$ tem bordo, denotaremos por $\log T_{\text {abs }}(W)$ o número definido na equação (2.11) com as formas satisfazendo a condição de contorno absoluta $\left(\mathcal{B}_{\text {abs }}\right)$ no bordo e por $\log T_{\text {rel }}(W)$ o número definido pela mesmas equação com as formas satisfazendo a condição de contorno relativa $\left(\mathcal{B}_{\text {rel }}\right)$ no bordo.

No caso de uma variedade $W$ sem bordo podemos ainda escrever a Torção Analítica somente em função dos autovalores das formas fechadas ou dos autovalores das formas cofechadas usando a decomposição de Hodge. De fato, seja $\mathcal{E}_{\lambda}^{(q)}$ o autoespaço do autovalor $\lambda \neq 0$ na dimensão $q$, a decomposição de Hodge induz uma decomposição em $\mathcal{E}_{\lambda}^{(q)}$ de modo que,

$$
\mathcal{E}_{\lambda}^{(q)}=\mathcal{E}_{\lambda, \mathrm{f}}^{(q)} \oplus \mathcal{E}_{\lambda, \mathrm{cf}}^{(q)}
$$

onde $\mathcal{E}_{\lambda, \mathrm{f}}^{(q)}$ é o autoespaço do autovalor $\lambda$ formado apenas por formas fechadas e $\mathcal{E}_{\lambda, \mathrm{cf}}^{(q)}$ é o autoespaço do autovalor $\lambda$ formado apenas por formas cofechadas. Quando $\lambda \neq 0$ temos que $\mathcal{E}_{\lambda, \mathrm{f}}^{(q)}\left(\mathcal{E}_{\lambda, \mathrm{cf}}^{(q)}\right)$ é igual o autoespaço do autovalor $\lambda$ formado apenas por formas exatas (coexatas) e este espaço será denotado por $\mathcal{E}_{\lambda, \text { ex }}^{(q)}\left(\mathcal{E}_{\lambda, \text { cex }}^{(q)}\right)$. Então,

$$
\zeta\left(s, \Delta^{(q)}\right)=\sum_{\lambda \in \operatorname{Sp}_{+} \Delta^{(q)}} \lambda^{-s}=\zeta_{\mathrm{f}}\left(s, \Delta^{(q)}\right)+\zeta_{\mathrm{cf}}\left(s, \Delta^{(q)}\right)
$$


onde

$$
\zeta_{\mathrm{f}}\left(s, \Delta^{(q)}\right)=\sum_{\lambda \in \mathrm{Sp}_{+} \Delta^{(q)}} \operatorname{dim} \mathcal{E}_{\lambda, \mathrm{f}}^{(q)} \lambda^{-s} \mathrm{e} \zeta_{\mathrm{cf}}\left(s, \Delta^{(q)}\right)=\sum_{\lambda \in \mathrm{Sp}_{+} \Delta^{(q)}} \operatorname{dim} \mathcal{E}_{\lambda, \mathrm{cf}}^{(q)} \lambda^{-s} .
$$

Agora, considerando a isometria $\lambda^{-\frac{1}{2}} d: \mathcal{E}_{\lambda, \mathrm{cf}}^{(q)} \rightarrow \mathcal{E}_{\lambda, \mathrm{f}}^{(q+1)}$, que tem inversa $\lambda^{-\frac{1}{2}} d^{\dagger}: \mathcal{E}_{\lambda, \mathrm{f}}^{(q+1)} \rightarrow$ $\mathcal{E}_{\lambda, \mathrm{cf}}^{(q)}$, vemos que

$$
\log T(W)=\frac{1}{2} \sum_{q=1}^{m}(-1)^{q} q \zeta^{\prime}\left(0, \Delta^{(q)}\right)=\frac{1}{2} \sum_{q=1}^{m}(-1)^{q} \zeta_{\mathrm{f}}^{\prime}\left(0, \Delta^{(q)}\right)=\frac{1}{2} \sum_{q=0}^{m-1}(-1)^{q+1} \zeta_{\mathrm{cf}}^{\prime}\left(0, \Delta^{(q)}\right) .
$$

Note que se a dimensão de $W$ é ímpar, $m$ é igual a $2 p-1$, considerando as isometrias

$$
\begin{aligned}
& \lambda^{-\frac{1}{2}} d *: \mathcal{E}_{\lambda, \mathrm{f}}^{(q)} \rightarrow \mathcal{E}_{\lambda, \mathrm{f}}^{(2 p-q)} \\
& \lambda^{-\frac{1}{2}} d^{\dagger} *: \mathcal{E}_{\lambda, \mathrm{cf}}^{(q)} \rightarrow \mathcal{E}_{\lambda, \mathrm{cf}}^{(2 p-2-q)}
\end{aligned}
$$

obtemos que

$$
\begin{aligned}
\log T(W) & =\sum_{q=1}^{p-1}(-1)^{q} \zeta_{\mathrm{f}}^{\prime}\left(0, \Delta^{(q)}\right)+\frac{1}{2}(-1)^{p} \zeta_{\mathrm{f}}^{\prime}\left(0, \Delta^{(p)}\right) \\
& =\sum_{q=0}^{p-2}(-1)^{q+1} \zeta_{\mathrm{cf}}^{\prime}\left(0, \Delta^{(q)}\right)+\frac{1}{2}(-1)^{p} \zeta_{\mathrm{cf}}^{\prime}\left(0, \Delta^{(p-1)}\right)
\end{aligned}
$$

Algumas propriedades que a Torção de Reidemeister satisfaz, a Torção Analítica também satisfaz. Apresentaremos apenas duas destas propriedades, para mais informações a respeito ver [36]. O primeiro resultado (que é bem interessante) para a Torção Analítica, e que pode ser encontrado em [27], é a Dualidade de Poincaré para a Torção Analítica. Como decorrência temos uma das propriedades, que a Torção de Reidemeister também satisfaz e foi provada por J.W. Milnor [29].

Proposição 4 (Dualidade de Poincaré para a Torção Analítica). Seja $W$ uma variedade Riemanniana de dimensão $m$, compacta, orientável e com bordo. Se $E_{\rho}$ é o fibrado vetorial associado a representação $\rho: \pi_{1}(W) \rightarrow O(n)$ então

$$
\log T_{\text {abs }}(W)=(-1)^{m+1} \log T_{\text {rel }}(W) .
$$

Demonstração. Consideremos uma métrica Riemanniana $g$ para $W$. Seja

$$
E_{\lambda}^{\mathrm{abs}}((W, g) ; \rho)^{q}:=\left\{\omega \in \Omega^{q}\left(M, E_{\rho}\right) \mid \Delta^{(q)} \omega=\lambda \omega, \mathcal{B}_{\mathrm{abs}}(\omega)=0\right\}
$$

e

$$
E_{\lambda}^{\mathrm{rel}}((M, g) ; \rho)^{q}:=\left\{\omega \in \Omega^{q}\left(M, E_{\rho}\right) \mid \Delta^{(q)} \omega=\lambda \omega, \mathcal{B}_{\mathrm{rel}}(\omega)=0\right\} .
$$

Defina

$$
E_{\lambda}^{\prime}((M, g) ; \rho)^{q}:=\left\{\omega \in \Omega^{q}\left(M, E_{\rho}\right) \mid d d^{\dagger} \omega=\lambda \omega, \mathcal{B}_{\mathrm{abs}}(\omega)=0\right\}
$$


e

$$
E_{\lambda}^{\prime \prime}((M, g) ; \rho)^{q}:=\left\{\omega \in \Omega^{q}\left(M, E_{\rho}\right) \mid d^{\dagger} d \omega=\lambda \omega, \mathcal{B}_{\mathrm{abs}}(\omega)=0\right\} .
$$

Para $\lambda \neq 0$ obtemos uma decomposição ortogonal

$$
\lambda^{-1} d d^{\dagger} \oplus \lambda^{-1} d^{\dagger} d: E_{\lambda}^{\mathrm{abs}}((M, g) ; \rho)^{q} \rightarrow E_{\lambda}^{\prime}((M, g) ; \rho)^{q} \oplus E_{\lambda}^{\prime \prime}((M, g) ; \rho)^{q}
$$

e um isomorfismo isométrico

$$
\begin{aligned}
& \lambda^{-\frac{1}{2}} d^{\dagger}: E_{\lambda}^{\prime}((M, g) ; \rho)^{q+1} \rightarrow E_{\lambda}^{\prime \prime}((M, g) ; \rho)^{q} \\
& \lambda^{-\frac{1}{2}} d: E_{\lambda}^{\prime \prime}((M, g) ; \rho)^{q} \rightarrow E_{\lambda}^{\prime}((M, g) ; \rho)^{q+1} .
\end{aligned}
$$

O operador $*$ de Hodge induz um isomorfismo isométrico

$$
*: E_{\lambda}^{\mathrm{abs}}((M, g) ; \rho)^{q} \rightarrow E_{\lambda}^{\mathrm{rel}}((M, g) ; \rho)^{m-q} .
$$

Agora usando estas informações obtemos

$$
\begin{aligned}
t_{\mathrm{abs}}(s) & =\sum_{q=0}^{m} \sum_{\lambda>0}(-1)^{q} q \lambda^{-s} \operatorname{dim} E_{\lambda}^{\mathrm{abs}}((M, g) ; \rho)^{q} \\
& =\sum_{q=0}^{m} \sum_{\lambda>0}(-1)^{q} q \lambda^{-s} \operatorname{dim} E_{\lambda}^{\mathrm{rel}}((M, g) ; \rho)^{m-q} \\
& =(-1)^{m} \sum_{q=0}^{m} \sum_{\lambda>0}(-1)^{m-q} q \lambda^{-s} \operatorname{dim} E_{\lambda}^{\mathrm{rel}}((M, g) ; \rho)^{m-q} \\
& =(-1)^{m+1} \sum_{q=0}^{m} \sum_{\lambda>0}(-1)^{m-q}(m-q) \lambda^{-s} \cdot \operatorname{dim} E_{\lambda}^{\mathrm{rel}}((M, g) ; \rho)^{m-q} \\
& +(-1)^{m} m \sum_{q=0}^{m} \sum_{\lambda>0}(-1)^{m-q} \lambda^{-s} \cdot \operatorname{dim} E_{\lambda}^{\mathrm{rel}}((M, g) ; \rho)^{m-q} \\
& =(-1)^{m+1} t_{\mathrm{rel}}(s) \\
& +(-1)^{m} m \sum_{q=0}^{m} \sum_{\lambda>0}(-1)^{m-q} \lambda^{-s} \cdot\left(\operatorname{dim} E_{\lambda}^{\prime}((M, g) ; \rho)^{q+1}+\operatorname{dim} E_{\lambda}^{\prime}((M, g) ; \rho)^{q}\right) \\
& =(-1)^{m+1} t_{\mathrm{rel}}(s) .
\end{aligned}
$$

E então

$$
\log T_{\text {abs }}(W)=(-1)^{m+1} \log T_{\text {rel }}(W) .
$$

Corolário 2. Suponha que $W$ seja uma variedade Riemanniana, compacta, orientável e sem bordo de dimensão par. Então $\log T(W)=0$.

A prova do último resultado da seção pode ser encontrado em [36]. 
Proposição 5. Suponha que $W_{1}$ e $W_{2}$ sejam duas variedades Riemannianas compactas orientáveis sem bordo, e que $W_{2}$ seja simplesmente conexa. Então a Torção Analítica do produto $W_{1} \times W_{2}$ é dada por

$$
\log T\left(W_{1} \times W_{2}\right)=\chi\left(W_{2}\right) \log T\left(W_{1}\right)
$$

\subsection{Motivação para a definição da Torção Analítica}

Apresentaremos aqui a justificativa original para a definição da Torção Analítica, que pode ser encontrada em [36].

Seja $W$ uma variedade Riemanniana compacta, orientável e sem bordo de dimensão $m$. Consideremos uma triangulação $K$ de $W$ e uma representação $\rho$ de $\pi_{1}(K)$ em $O(n)$ de maneira que $C\left(K ; E_{\rho}\right)$ seja acíclico. Notemos que a Torção de Reidemeister de $W$ pode ser representada em termos de determinantes formados a partir do operador bordo $\partial$ de $C\left(K ; E_{\rho}\right)$, como segue. A escolha de uma base preferida para cada $C_{q}$ nos permite representar $\partial: C_{q} \rightarrow C_{q-1}$ como uma matriz real. Seja $\partial^{*}: C_{q} \rightarrow C_{q+1}$ a matriz transposta de $\partial$, e defina o Laplaciano combinatorial $\Delta_{c}: C_{q} \rightarrow C_{q}$ por

$$
\Delta_{c}=\partial^{*} \partial+\partial \partial^{*}
$$

Seja $\Delta_{c}^{(q)}$ a matriz representando $\Delta_{c}$ em cada $C_{q}$. Como $C\left(K ; E_{\rho}\right)$ é acíclico, $\Delta_{c}^{(q)}$ é não singular para cada $q$.

Proposição 6. Se a Torção de Reidemeister $\tau(W)$ está definida para uma representação $\rho$, então

$$
\log \tau(W)=\frac{1}{2} \sum_{q=0}^{m}(-1)^{q+1} q \log \operatorname{det}\left(\Delta_{c}^{(q)}\right) .
$$

Demonstração. Em cada $C_{q}\left(K ; E_{\rho}\right)$, a base preferida determina um produto interno de maneira que cada Laplaciano combinatorial é simétrico e uma matriz estritamente positiva. Mais ainda, como $\partial^{2}=0$, o subespaço $B_{q}=\partial\left(C_{q+1}\right)$ é invariante sobre $\Delta_{c}^{(q)}$. Seja $\mathbf{b}_{q}=$ $\left(b_{1}^{(q)}, \ldots, b_{r_{q}}^{(q)}\right)$ uma base ortonormal para $B_{q}$ consistindo dos autovetores de $\Delta_{c}^{(q)}$, então

$$
\Delta_{c}^{(q)} b_{j}^{(q)}=\partial \partial^{*} b_{j}^{(q)}=\lambda_{j}^{(q)} b_{j}^{(q)}
$$

Escolhida esta base para cada $B_{q}$, defina

$$
\tilde{b}_{j}^{(q-1)}=\frac{1}{\lambda_{j}^{(q-1)}} \partial^{*} b_{j}^{(q-1)}, \quad j=1, \ldots, r_{q-1}
$$

e $\operatorname{assim} \partial \tilde{b}_{j}^{(q-1)}=b_{j}^{(q-1)}$. Observe que os vetores $\tilde{b}_{j}^{(q-1)}$ são ortogonais, com

$$
\left\|\tilde{b}_{j}^{(q-1)}\right\|^{2}=\frac{1}{\left(\lambda_{j}^{(q-1)}\right)^{2}}\left\langle\partial^{*} b_{j}^{(q-1)}, \partial^{*} b_{j}^{(q-1)}\right\rangle=\frac{1}{\lambda_{j}^{(q-1)}} .
$$


Logo

$$
\begin{aligned}
\log \tau(W) & =\sum_{q=0}^{m}(-1)^{q} \log \left[\mathbf{b}_{q}, \tilde{\mathbf{b}}_{q-1} / c_{q}\right] \\
& =\sum_{q=1}^{m}(-1)^{q} \log \prod_{1}^{r_{q-1}}\left(\lambda_{j}^{(q-1)}\right)^{\frac{1}{2}}
\end{aligned}
$$

Mas a base ortonormal de $C_{q}$ formada por $b_{j}^{(q)}, j=1, \ldots, r_{q}$ e $\left(\lambda_{j}^{(q)}\right)^{\frac{1}{2}} \tilde{b}^{(q-1)_{j}}, j=1, \ldots, r_{q-1}$, diagonaliza $\Delta_{c}^{(q)}$, tal que,

$$
\operatorname{det}\left(\Delta_{c}^{(q)}\right)=\prod_{1}^{r_{q}}\left(\lambda_{j}^{(q)}\right) \prod_{1}^{r_{q-1}}\left(\lambda_{j}^{(q-1)}\right) .
$$

Isto implica que

$$
\log \prod_{1}^{r_{q-1}}\left(\lambda_{j}^{(q-1)}\right)=\sum_{k=q}^{m}(-1)^{k-q} \log \operatorname{det}\left(\Delta_{c}^{(k)}\right),
$$

que nos leva a expressão no enunciado da proposição.

Agora observamos que o determinante da matriz não singular $\Delta_{c}^{(q)}$ pode ser expresso em termos de sua função zeta

$$
\zeta\left(s, \Delta_{c}^{(q)}\right)=\frac{1}{\Gamma(s)} \int_{0}^{\infty} t^{s-1} \operatorname{Tr}\left(e^{t \Delta_{c}^{(q)}}\right) d t=\operatorname{Tr}\left(\Delta_{c}^{(q)}\right)^{-s} .
$$

Ainda, como para cada autovalor $\lambda$ de $\Delta_{c}^{(q)}$ tem-se

$$
\frac{d}{d s}(\lambda)^{-s}=-\log (\lambda)(\lambda)^{-s}
$$

temos $\log \operatorname{det}\left(\Delta_{c}^{(q)}\right)=-\left.\frac{d}{d s} \zeta\left(s, \Delta_{c}^{(q)}\right)\right|_{s=0}$. Assim, a fórmula para a Torção Analítica é um análogo formal para a Torção de Reidemeister, já que

$$
\log \tau(W)=\frac{1}{2} \sum_{q=0}^{m}(-1)^{q} q \zeta^{\prime}\left(0, \Delta_{c}^{(q)}\right) .
$$

\subsection{Teoremas de comparação}

Em vista da relação entre os complexos $\Omega\left(W ; E_{\rho}\right)$ e $C\left(K ; E_{\rho}\right)$ dada pelo Teorema de de Rham e as propriedades que tanto a Torção Analítica quanto a Torção de Reidemeister satisfazem, D.B. Ray e I.M. Singer conjecturaram que a Torção Analítica e a Torção de Reidemeister eram iguais, mas não conseguiram uma prova. Anos mais tarde, J. Cheeger [6] e W. Müller [32] provaram de maneira independente o teorema a seguir:

Teorema 7 (Teorema de Cheeger Müller). Se $W$ é uma variedade Riemanniana fechada e $\rho$ uma representação de $\pi_{1}(W)$ em $O(n)$ então $\tau(W)=T(W)$. 
Quando $W$ é uma variedade com bordo, J. Cheeger em [6] observa (mas sem prova) que o Teorema de Cheeger Müller ainda vale, mas termos dependendo do bordo aparecem, ou seja,

$$
\log T(W)=\log \tau(W)+f(\partial W)
$$

Um primeiro resultado para o termo do bordo $f(\partial W)$ foi apresentado por W. Lück [27] no caso em que a a métrica da variedade têm estrutura produto próximo ao bordo, ou seja,

Definição 22. Seja $W$ uma variedade Riemanniana compacta com bordo $\partial W$. Diremos que a métrica $g$ de $W$ é produto próxima ao bordo se existir um colar $f: \partial W \times[0,1) \rightarrow U \subset W$, onde $U$ é uma vizinhança de $W$ de maneira que $\partial W \subset U$, tal que $f$ é uma isometria se, $[0,1)$ tem a métrica padrão, em $\partial W \times[0,1)$ a métrica é a produto e a métrica em $U$ é a induzida por $W$.

Neste caso, o termo do bordo $f(\partial W)$ é puramente topológico e é proporcional a característica de Euler do bordo, mais precisamente:

Teorema 8. Se $W$ é uma variedade Riemanniana compacta com bordo $\partial W$ de dimensão $m$, cuja métrica tem estrutura produto próxima ao bordo, então, no caso absoluto,

$$
\log T_{\mathrm{abs}}(W)=\log \tau(W)+\frac{1}{4} \chi(\partial W) \log 2 .
$$

Mais recentemente, o termo do bordo foi estudado no caso geral por X. Dai e H. Fang [13] e por J. Brüning e X. Ma [4]. Eles provaram a existência de mais uma contribuição não topológica quando a métrica não é produto proximo ao bordo, a saber,

$$
\log T_{\mathrm{abs}}(W)=\log \tau(W)+\frac{1}{4} \chi(\partial W) \log 2+A(\partial W) .
$$

O termo $A(\partial W)$ será chamado de termo anômalo do bordo. Em relação ao trabalho de X. Dai e H. Fang [13] apresentaremos um contra exemplo no capítulo 5 mostrando a existência de algum problema no teorema principal deste artigo. Apresentaremos aqui o resultado de [4] que será usado no decorrer do trabalho.

Sabemos que dado um espaço vetorial $V$, existe uma involução em $V$ de modo que $V=$ $V_{+} \oplus V_{-}$. Logo $V$ é um espaço vetorial $\mathbb{Z}_{2}$ graduado. Seja $A$ uma algebra exterior em $V$ com produto tal que, $A_{j} A_{k} \subset A_{j+k}$. Chamaremos $A$ de uma algebra $\mathbb{Z}_{2}$ graduada. Um exemplo deste fato é uma algebra graduada

$$
(\Lambda V)_{ \pm}=\sum_{(-1)^{k}= \pm 1} \Lambda^{k} V
$$

Sejam $A$ e $B$ duas $\mathbb{Z}_{2}$ algebras graduadas. O produto tensorial $A \otimes B$ possui uma $\mathbb{Z}_{2}$ graduação natural que denotaremos por $A \hat{\otimes} B=(A \otimes B)_{+} \oplus(A \otimes B)_{-}$, onde $(A \otimes B)_{+}=A_{+} \otimes B_{+} \oplus$ $A_{-} \otimes B_{-}$e $(A \otimes B)_{-}=A_{+} \otimes B_{-} \oplus A_{-} \otimes B_{+}$. Identificaremos $A$ com $A \hat{\otimes} 1$ e denotaremos por 
$\hat{B}=1 \hat{\otimes} B$, e escreveremos $\wedge:=\hat{\otimes}$. Então, $A \hat{\otimes} B=A \hat{\otimes} 1 \wedge 1 \hat{\otimes} B=A \wedge \hat{B}$. Observamos que o produto em $A \wedge \hat{B}$ é da forma

$$
\left(a_{1} \wedge \hat{b}_{1}\right) \wedge\left(a_{2} \wedge \hat{b}_{2}\right)=(-1)^{\left|a_{2}\right|\left|b_{1}\right|}\left(a_{1} \wedge a_{2}\right) \wedge \widehat{b_{1} \wedge b_{2}}
$$

onde $\left|a_{2}\right|=\operatorname{dim} a_{2}$ e $\left|\hat{b}_{1}\right|=\operatorname{dim} \hat{b}_{1}$ (para mais informações ver [1]).

Para dois espaço vetoriais reais $V$ e $E$, de dimensão $k$ e $n$, assuma que $E$ é euclidiano e orientado com base ortonormal $\left\{e_{j}\right\}_{j=1}^{n}$ e base dual $\left\{\hat{e}^{j}\right\}_{j=1}^{n}$, com respeito a métrica euclidiana e denote por $\Lambda E^{*}$ a algebra exterior de $E^{*}$. Então a integral de Berezin é a aplicação linear

$$
\begin{aligned}
& \int^{B}: \Lambda V^{*} \hat{\otimes} \Lambda E^{*} \rightarrow \Lambda V^{*}, \\
& \int^{B}: \alpha \hat{\otimes} \beta \mapsto \frac{(-1)^{\frac{n(n+1)}{2}}}{\pi^{\frac{n}{2}}} \beta\left(e_{1}, \ldots, e_{n}\right) \alpha,
\end{aligned}
$$

onde $\left\{e_{j}\right\}_{j=1}^{n}$ é uma base ortonormal de $E$.

Seja $A$ um endomorfismo anti-simétrico de E, identificaremos $A$ com um elemento de $\Lambda E^{*}$ por

$$
\text { ^ }: A \mapsto \hat{A}=\frac{1}{2} \sum_{j, l=1}^{n}\left(e_{j}, A e_{l}\right) \hat{e}^{j} \wedge \hat{e}^{l} .
$$

Como um exemplo da integral de Berezin temos

$$
\int^{B} \mathrm{e}^{-\frac{\hat{A}}{2}}=\operatorname{Pf}\left(\frac{A}{2 \pi}\right)
$$

que é nula se $\operatorname{dim} E$ é impar.

Lembrando a cisão do fibrado tangente $T W$ sobre o colar $C(\partial W)=(-\epsilon, 0] \times \partial W$ apresentado na seção 1 deste capítulo, definimos a 1 -forma $s_{j}$ com valores nos endomorfismos anti-adjuntos de $T W$ por

$$
s_{j}=\nabla_{j}^{T W}-\nabla^{T C(\partial W)}=\left(\nabla_{j}^{T W} P_{\mathrm{tg}}\right)_{\text {norm }}+\left(\nabla_{j}^{T W} P_{\text {norm }}\right)_{\mathrm{tg}},
$$

onde $\nabla_{j}^{T W}$ é a conexão de Levi-Civita em $\left(W, g_{j}\right), \nabla^{T C(\partial W)}$ é a conexão de Levi-Civita em $C(\partial W)$ com a métrica produto e $P$ denota a projeção associada à cisão $T C(\partial W)=$ $N \partial W \oplus T \partial W$. Com a notação anterior definimos

$$
\begin{aligned}
\mathcal{S}_{j} & =\frac{1}{2} \sum_{k=1}^{m-1}\left(i^{*} s_{j}\left(e_{k}\right)\right)_{\mathrm{norm}} \hat{e}^{k}, \\
\mathcal{R} & =\hat{\tilde{R}}
\end{aligned}
$$

onde $\left(e_{y_{1}}, \ldots, e_{y_{m-1}}\right)$ é uma base ortonormal em $T \partial W,\left(e^{y_{1}}, \ldots, e^{y_{m-1}}\right)$ a base ortonormal dual de $\left(e_{y_{1}}, \ldots, e_{y_{m-1}}\right)$ e $\tilde{R}$ é a 2 -forma curvatura do $\partial W$.

\section{Definição 23.}

$$
B\left(\nabla_{j}^{T W}\right)=\frac{1}{2} \int_{0}^{1} \int^{B} \mathrm{e}^{-\frac{1}{2} \mathcal{R}-u^{2} \mathcal{S}_{j}^{2}} \sum_{k=1}^{\infty} \frac{1}{\Gamma\left(\frac{k}{2}+1\right)} u^{k-1} \mathcal{S}_{j}^{k} d u
$$


Agora para obter o termo anômalo do bordo $A(\partial W)$ usaremos o seguinte teorema, que está em [4].

Teorema 9 (Comparação entre Torções Analíticas). Seja W uma variedade Riemanniana compacta, com bordo $\partial W$, orientável com duas métricas $g_{0}$ e $g_{1}$. Então o logaritmo da razão entre $T_{\mathrm{abs}}\left(W, g_{1}\right)$ e $T_{\mathrm{abs}}\left(W, g_{0}\right)$ é da forma

$$
\log \frac{T_{\mathrm{abs}}\left(W, g_{1}\right)}{T_{\mathrm{abs}}\left(W, g_{0}\right)}=\frac{1}{2} \int_{\partial W}\left(B\left(\nabla_{1}^{T W}\right)-B\left(\nabla_{0}^{T W}\right)\right),
$$

onde $\nabla_{j}^{T W}$ é a conexão de Levi-Civita da métrica $g_{j}$ e as formas $B\left(\nabla_{j}^{T W}\right)$ foram definidas anteriormente.

Assim para obtermos a comparação entre a Torção Analítica e a Torção de Reidemeister procedemos da seguinte maneira: Considere $g_{1}=g$ (definida em (2.4) ) e $g_{0}$ uma oportuna deformação de $g$ que é produto próximo ao bordo, a saber,

$$
g_{0}=d r \otimes d r+g_{\partial W}
$$

tal que $g_{\partial W}=i^{*} g$ e $i: \partial W \hookrightarrow W$ é a inclusão. Pelo teorema 9, temos

$$
\log T_{\mathrm{abs}}(W)=\log T_{\mathrm{abs}}\left(W, g_{0}\right)+\frac{1}{2} \int_{\partial W}\left(B\left(\nabla_{1}^{T W}\right)-B\left(\nabla_{0}^{T W}\right)\right) .
$$

Usando o teorema 8 em $\log T_{\text {abs }}\left(W, g_{0}\right)$, temos

$$
\log T_{\mathrm{abs}}(W)=\log \tau(W)+\frac{1}{4} \chi(\partial W) \log 2+\frac{1}{2} \int_{\partial W}\left(B\left(\nabla_{1}^{T W}\right)-B\left(\nabla_{0}^{T W}\right)\right) .
$$

Notamos que na linguagem dos fibrados e das conexões, se $\omega_{j}$ denota a 1-forma conexão associada a métrica $g_{j}$, e $\Theta$ é a 2-forma curvatura do bordo, usando a notação $M_{b}^{a}$ para a entrada com linha $a$ e coluna $b$ da matriz $M$, as equações (2.15) possuem a forma

$$
\begin{gathered}
\mathcal{S}_{j}=\frac{1}{2} \sum_{k=1}^{m-1}\left(i^{*} \omega_{j}-i^{*} \omega_{0}\right)^{r} y_{k} \hat{e}^{y_{k}}, \\
\mathcal{R}=\hat{\Theta}=\frac{1}{2} \sum_{k, l=1}^{m-1} \Theta^{y_{k}} \hat{y}_{l} \hat{e}^{y_{k}} \wedge \hat{e}^{y_{l}} .
\end{gathered}
$$

Logo $\mathcal{S}_{0}$ é nula e vale a igualdade

$$
\mathcal{R}=\left.\hat{R}\right|_{\partial W}-2 \mathcal{S}_{1}^{2}
$$

que é a equação (1.16) de [4], onde $R$ é a 2 -forma curvatura de $W$. Então temos o teorema:

Teorema 10 (Teorema de Cheeger Müller para variedades com bordo). Nas condições acima, a comparação entre a Torção Analítica e a Torção de Reidemeister é dada pela fórmula

$$
\log T_{\text {abs }}(W)=\log \tau(W)+\frac{1}{4} \chi(\partial W) \log 2+\frac{1}{2} \int_{\partial W} B\left(\nabla_{1}^{T W}\right) .
$$




\title{
Capítulo 3
}

\section{Torção Analítica de variedades com bordo totalmente geodésico}

\begin{abstract}
Neste capítulo determinaremos a Torção Analítica de variedades com bordo totalmente geodésico, um resultado que estende o teorema 8. E ainda, faremos a Torção Analítica para o caso particular da meia-esfera, como uma aplicação deste resultado. No intuito de demonstrar o quanto este último processo é mais complicado que o método geométrico, apresentaremos também o cálculo explicito da Torção Analítica no caso da calota esférica.
\end{abstract}

\subsection{Variedades com o bordo totalmente geodésico}

Seja $(M, g)$ uma variedade Riemanniana conexa de dimensão $m$ e métrica $g$.

Definição 24. Uma subvariedade $M$ de $W$ é totalmente geodésica se as geodésicas de $M$ são geodésicas em $W$, ou seja, se $i: M \rightarrow W$ denota a inclusão, $M$ é totalmente geodésica se, $e$ somente se, a imersão isométrica $i:\left(M, i^{*} g\right) \rightarrow(W, g)$ é totalmente geodésica.

De uma maneira mais simples, a subvariedade $M$ ser totalmente geodésica significa que qualquer geodésica de $\left(M, i^{*} g\right)$ é levada por $i$ em uma geodésica de $(W, g)$. Se $M=\partial W$ é o bordo de $W$, e $\partial W$ é totalmente geodésico, diremos que $W$ possue bordo totalmente geodésico.

Apresentaremos uma condição local para uma variedade ter um bordo totalmente geodésico. Como anteriormente, seja $\partial_{r}$ denotando o vetor unitário normal ao bordo apontando para fora, isto é, um base ortonormal local para $N \partial W$, o fibrado normal. Próximo ao bordo temos a decomposição $\mathcal{C}(\partial W)=(-\epsilon, 0] \times \partial W$, obtido usando o fluxo geodésico interno. Se $y=\left(y_{1}, \ldots, y_{m-1}\right)$ é um sistema local de coordenadas no bordo, então $(r, y)$ é um sistema de coordenadas local em $\mathcal{C}(\partial W)$. Aqui as curvas $r \rightarrow(r, y)$ são geodésicas com velocidade unitária perpendicular ao bordo. Nesse sistema de coordenadas locais, o tensor métrico tem 
a forma de (2.4), mais precisamente

$$
g=d r \otimes d r+\tilde{g}(r)
$$

Seja $\Gamma_{\alpha \beta}^{\gamma}$ denotando os símbolos de Christoffel relativos a base coordenada associada ao sistema de coordenada local $(r, y)$ em $(W, g)$, e $\tilde{\Gamma}_{j k}^{l}$ denota o símbolo de Christoffel relativo a base coordenada associada ao sistema de coordenada local $(y)$ em $(\partial W, \tilde{g}(0))$. Então a equação geodésica (1.7) em $(W, g)$

$$
\partial_{t}^{2} f^{\gamma}+\Gamma_{\alpha}^{\gamma}{ }_{\beta} \partial_{t} f^{\alpha} \partial_{t} f^{\beta}=0
$$

com $\gamma=y_{1}, y_{2}, \ldots, y_{m-1}$ e $f: I \rightarrow W$ uma curva parametrizada, corresponde ao sistema de equações

$$
\left\{\begin{array}{l}
\partial_{t}^{2} f^{r}-\frac{1}{2} \partial_{r} \tilde{g}_{y_{j} y_{k}}(r) \partial_{t} f^{y_{j}} \partial_{t} f^{y_{k}}=0, \\
\partial_{t}^{2} f^{y_{l}}+\tilde{\Gamma}_{j k}^{l} \partial_{t} f^{y_{j}} \partial_{t} f^{y_{k}}+\tilde{g}^{y_{l} y_{k}}(r) \partial_{r} \tilde{g}_{y_{k} y_{h}}(r) \partial_{t} f^{r} \partial_{t} f^{y_{h}}=0 .
\end{array}\right.
$$

Isto mostra que uma geodésica de $\left(\partial W, i^{*} g\right)$ é uma geodésica em $(W, g)$ se, e somente se, $\partial_{r} \tilde{g}(r)=0$. Assim, temos o seguinte resultado.

Lema 4. Seja $(W, g)$ uma variedade Riemanniana conexa com bordo $\partial W$. Seja $(r, y)$ um sistema de coordenadas local próximo ao bordo, onde $r$ é a distancia geodésica do bordo e a métrica $g$ é da forma $g=d r \otimes d r+\tilde{g}(r)$. Então, $\partial W$ é totalmente geodésica se, e somente se, $\partial_{r} \tilde{g}(r)=0$.

Interpretaremos este resultado usando a segunda forma fundamental de $\partial W$, que definiremos aqui. Seja $M$ uma subvariedade de $(W, g)$ e $i: M \rightarrow W$ a inclusão. A restrição $\left.T W\right|_{M}$ do fibrado tangente de $W$ sobre $M$ decompõe-se como a soma de Whitney $T M \oplus N M$, onde $N M$ é o fibrado normal de $M$. Para um vetor $v$ de $\left.T W\right|_{M}$ denote por $v_{\tan }$ e $v_{\text {norm }}$ as componentes tangencial e normal, respectivamente. Seja $\nabla$ a conexão de Levi-Civita de $(W, g)$.

Definição 25. Dado duas seções $u, v \in \Gamma(M, T M)$ de $T M$, a segunda forma fundamental $S_{M}$ de $M$ é definida por

$$
S_{M}(u, v)=\left(\nabla_{u} v\right)_{\text {norm }} .
$$

Dessa forma, $W$ tem bordo totalmente geodésica se, e somente se, a segunda forma fundamental de $\partial W$ é identicamente nula. Para isto basta usar a descrição local da segunda forma fundamental como o $(0,2)$-tensor simétrico $S_{\partial W}\left(y_{j}, y_{k}\right)=\Gamma_{y_{j}{ }^{r}{ }_{k}} g_{r r}=\Gamma_{y_{j} r y_{k}}=-\frac{1}{2} \partial_{r} \tilde{g}_{y_{j} y_{k}}$ e o lema 4 segue.

Com o que foi apresentado até agora estamos pronto para provar,

Teorema 11. Seja $W$ uma variedade Riemanniana compacta, conexa, orientável de dimensão $m$ com o bordo $\partial W$ totalmente geodésico. Então

$$
(-1)^{m-1} \log T_{\text {rel }}(W)=\log T_{\text {abs }}(W)=\log \tau(W)+\frac{1}{4} \chi(\partial W) \log 2,
$$

onde $\chi(\partial W)$ é a característica de Euler do bordo. 
Demonstração. Em vista do teorema 10 basta mostrar que

$$
\frac{1}{2} \int_{\partial W} B\left(\nabla_{1}^{T W}\right)=0
$$

Lembrando a equação (2.16) temos

$$
B\left(\nabla_{1}^{T W}\right)=\frac{1}{2} \int_{0}^{1} \int^{B} \mathrm{e}^{-\frac{1}{2} \mathcal{R}-u^{2} \mathcal{S}_{1}^{2}} \sum_{k=1}^{\infty} \frac{1}{\Gamma\left(\frac{k}{2}+1\right)} u^{k-1} \mathcal{S}_{1}^{k} d u,
$$

e mostrando que $\mathcal{S}_{1}=0$ teremos o resultado. Seguindo esta idéia, na equação (2.15), vemos que $\mathcal{S}_{1}$ é a restrição da forma $s$ ao bordo, que é precisamente a segunda forma fundamental $S_{\partial W}$ no bordo, logo $\mathcal{S}_{1}$ é nula. Uma outra maneira para ver que $\mathcal{S}_{1}=0$, segue usando a fórmula na equação (2.17) e lembrando que $\left(i^{*} \omega_{1}\right)^{r} y_{k}=\Gamma_{y_{j}}{ }^{r} y_{k} e^{y_{j}}$ que é nulo pelo lemma 4. Desta forma temos que

$$
\log T_{\mathrm{abs}}(W)=\log \tau(W)+\frac{1}{4} \chi(\partial W) \log 2 .
$$

Para obtermos a primeira igualdade basta usar a Dualidade de Poincaré para a Torção Analítica, proposição 4 .

\subsection{Torção Analítica das meia-esferas}

Calcularemos nesta seção a Torção analítica para as meia-esferas usando o teorema 11, que está na seção anterior. Como estes espaços são variedades simplesmente conexas, a representação do grupo fundamental entra apenas como a dimensão do espaço de representação. Seja $\mathbb{S}_{l}^{m}$ denotando a meia-esfera de dimensão $m$ e raio $l$,

$$
\mathbb{S}_{l}^{m}=\left\{x \in \mathbb{R}^{m+1}|| x \mid=l, x_{m+1} \geq 0\right\} .
$$

O bordo de $\mathbb{S}_{l}^{m}$ é a esfera $S_{l}^{m-1}=\left\{x \in \mathbb{R}^{m+1}|| x \mid=l, x_{m+1}=0\right\}$. Parametrizamos $\mathbb{S}_{l}^{m}$ por

$$
\mathbb{S}_{l}^{m}=\left\{\begin{aligned}
x_{1} & =l \operatorname{sen} \theta_{m} \operatorname{sen} \theta_{m-1} \cdots \operatorname{sen} \theta_{3} \operatorname{sen} \theta_{2} \cos \theta_{1} \\
x_{2} & =l \operatorname{sen} \theta_{m} \operatorname{sen} \theta_{m-1} \cdots \operatorname{sen} \theta_{3} \operatorname{sen} \theta_{2} \operatorname{sen} \theta_{1} \\
x_{3} & =l \operatorname{sen} \theta_{m} \operatorname{sen} \theta_{m-1} \cdots \operatorname{sen} \theta_{3} \cos \theta_{2} \\
& \vdots \\
x_{m} & =l \operatorname{sen} \theta_{m} \cos \theta_{m-1} \\
x_{m+1} & =l \cos \theta_{m}
\end{aligned}\right.
$$

$\operatorname{com} \theta_{1} \in[0,2 \pi], \theta_{2}, \ldots, \theta_{m-1} \in[0, \pi]$ e $\theta_{m} \in\left[0, \frac{\pi}{2}\right]$. A métrica induzida é

$$
\begin{aligned}
g & =\operatorname{sen}^{2} \theta_{m} g_{S_{l}^{m-1}}+l^{2} d \theta_{m} \otimes d \theta_{m} \\
& =l^{2}\left(\sum_{j=1}^{m-1}\left(\prod_{j=i+1}^{m} \operatorname{sen}^{2} \theta_{j}\right) d \theta_{k} \otimes d \theta_{k}+d \theta_{m} \otimes d \theta_{m}\right),
\end{aligned}
$$


e $\sqrt{|\operatorname{det} g|}=l^{m}\left(\operatorname{sen} \theta_{m}\right)^{m-1}\left(\operatorname{sen} \theta_{m-1}\right)^{m-2} \cdots\left(\operatorname{sen} \theta_{3}\right)^{2}\left(\operatorname{sen} \theta_{2}\right)$.

Seja $K$ a decomposição celular de $\mathbb{S}_{l}^{m}$ com uma $m$-célula, uma $(m-1)$-célula e uma 0célula, $K=c_{m}^{1} \cup c_{m-1}^{1} \cup c_{0}^{1}$. Seja o subcomplexo $L$ de $K$ a decomposição celular de $\partial S_{l}^{m}$, $L=c_{m-1}^{1} \cup c_{0}^{1}$.

Consideraremos primeiro o caso com condições de contorno relativa no bordo. Então o complexo de espaços vetoriais (2.1) tem a forma

$$
C_{\mathrm{rel}}: 0 \longrightarrow \mathbb{R}\left[c_{m}^{1}\right] \longrightarrow 0 \longrightarrow 0 \longrightarrow 0 \longrightarrow 0
$$

com base preferida $\mathbf{c}_{m}=\left\{c_{m}^{1}\right\}$. Para fixar a base para a homologia, precisamos de uma base ortonormal graduada para as formas harmônicas. Como uma base para $\Omega^{m}\left(\mathbb{S}_{l}^{m}\right)$ é $\left\{\sqrt{|\operatorname{det} g|} d \theta_{1} \wedge \cdots \wedge d \theta_{m}\right\}$, seja $\mathbf{a}_{m}=\left\{\frac{\sqrt{|\operatorname{det} g|} d \theta_{1} \wedge \cdots \wedge d \theta_{m}}{\sqrt{\operatorname{Vol}_{g}\left(\mathbb{S}_{l}^{m}\right)}}\right\}$. Aplicando a fórmula da equação (2.8) obtemos $\mathbf{h}_{m}=\left\{h_{m}^{1}\right\}$, e

$$
\begin{aligned}
h_{m}^{1} & =\mathcal{A}_{m}^{\mathrm{rel}}\left(a_{m}^{1}\right)=\frac{1}{\sqrt{\operatorname{Vol}_{g}\left(\mathbb{S}_{l}^{m}\right)}} \int_{\mathrm{pt}} * \sqrt{|\operatorname{det} g|} d \theta_{1} \wedge \cdots \wedge d \theta_{m} c_{m}^{1} \\
& =\frac{1}{\sqrt{\operatorname{Vol}_{g}\left(\mathbb{S}_{l}^{m}\right)}} c_{m}^{1} .
\end{aligned}
$$

E ainda, $\mathbf{b}_{q}=\emptyset$ para todo $q, \operatorname{logo}$

$$
\left|\operatorname{det}\left(\mathbf{h}_{m} / \mathbf{c}_{m}\right)\right|=\frac{1}{\sqrt{\operatorname{Vol}_{g}\left(\mathbb{S}_{l}^{m}\right)}}, \quad\left|\operatorname{det}\left(\mathbf{b}_{q} / \mathbf{c}_{q}\right)\right|=1,0 \leq q \leq m-1 .
$$

Com isso, utilizando as definições nas equações (2.9) e (2.2), provamos o resultado a seguir.

Proposição 7. $\tau\left(\mathbb{S}_{l}^{m}, S_{l}^{m-1}\right)=\left(\sqrt{\operatorname{Vol}_{g}\left(\mathbb{S}_{l}^{m}\right)}\right)^{(-1)^{m-1} \mathrm{rk}(\rho)}=\left(\frac{l^{m} \frac{m+1}{2}}{\Gamma\left(\frac{m+1}{2}\right)}\right)^{\frac{\mathrm{rk}(\rho)}{2}(-1)^{m-1}}$.

Agora consideraremos o caso com condições de contorno absoluta no bordo. Pela equação (2.1), o complexo relevante é

$$
C_{\text {abs }}: 0 \longrightarrow \mathbb{R}\left[c_{m}^{1}\right] \longrightarrow \mathbb{R}\left[c_{m-1}^{1}\right] \longrightarrow 0 \longrightarrow 0 \longrightarrow 0\left[c_{0}^{1}\right] \longrightarrow 0,
$$

com base preferida $\mathbf{c}_{m}=\left\{c_{m}^{1}\right\}, \mathbf{c}_{m-1}=\left\{c_{m-1}^{1}\right\}$ e $\mathbf{c}_{0}=\left\{c_{0}^{1}\right\}$. Como, $H_{p}(K)=0$, para $p>1$ e $H_{0}(K)=\mathbb{R}\left[c_{0}^{1}\right]$, e ainda uma base para $\Omega^{0}\left(\mathbb{S}_{l}^{m}\right)$ é a forma constante $\{1\}$, logo $\mathbf{a}_{0}=\left\{\frac{1}{\sqrt{\operatorname{Vol}_{g}\left(\mathbb{S}_{l}^{m}\right)}}\right\}$. Aplicando a fórmula da equação (2.8) obtemos $\mathbf{h}_{0}=\left\{h_{0}^{1}\right\}$, com

$$
\begin{aligned}
h_{0}^{1} & =\mathcal{A}_{0}^{\mathrm{abs}}\left(a_{0}^{1}\right)=\frac{1}{\sqrt{\operatorname{Vol}_{g}\left(\mathbb{S}_{l}^{m}\right)}} \int_{\mathbb{S}_{l}^{m}} * 1 c_{0}^{1} \\
& =\sqrt{\operatorname{Vol}_{g}\left(\mathbb{S}_{l}^{m}\right)} c_{0}^{1} .
\end{aligned}
$$

Como $\mathbf{b}_{q}=\emptyset$ para $q=0, \ldots, m-1, \tilde{b}_{m-1}^{1}=c_{m}^{1}, b_{m}^{1}=c_{m-1}^{1}$ temos que

$$
\begin{array}{rlrl}
\left|\operatorname{det}\left(\mathbf{h}_{0} / \mathbf{c}_{0}\right)\right| & =\sqrt{\operatorname{Vol}_{g}\left(\mathbb{S}_{l}^{m}\right)}, & \\
\left|\operatorname{det}\left(\mathbf{b}_{m} / \mathbf{c}_{m-1}\right)\right| & =1, & & \left|\operatorname{det}\left(\tilde{\mathbf{b}}_{m-1} / \mathbf{c}_{m}\right)\right|=1 .
\end{array}
$$

Aplicando a definição nas equações (2.10) e (2.2), provamos o seguinte resultado 
Proposição 8. $\tau\left(\mathbb{S}_{l}^{m}\right)=\left({\sqrt{\operatorname{Vol}_{g}\left(\mathbb{S}_{l}^{m}\right)}}^{\mathrm{rk}(\rho)}=\left(\frac{l^{m} \pi \frac{m+1}{2}}{\Gamma\left(\frac{m+1}{2}\right)}\right)^{\frac{\mathrm{rk}(\rho)}{2}}\right.$.

No caso absoluto, basta usar o teorema 11 para obtermos:

\section{Corolário 3.}

$$
\begin{aligned}
\log T_{\mathrm{abs}}\left(\mathbb{S}_{l}^{m}, g\right) & =\frac{\operatorname{rk}(\rho)}{2} \log \operatorname{Vol}_{g}\left(\mathbb{S}_{l}^{m}\right)+\frac{1}{4} \chi\left(S^{m-1}\right) \log 2 \\
& =\frac{\operatorname{rk}(\rho)}{2} \log \frac{l^{m} \pi^{\frac{m+1}{2}}}{\Gamma\left(\frac{m+1}{2}\right)}+\frac{1}{4}((m-1) \bmod 2) \log 2
\end{aligned}
$$

\subsection{Torção Analítica da calota esférica}

Nesta seção investigaremos o problema de obter a Torção Analítica por meio da aplicação direta da definição. Este é um exemplo da aplicabilidade das ferramentas analíticas na geometria (o que era o objetivo principal de Ray e Singer), e um bom exemplo do cálculo explícito da Torção Analítica, o que quase não se encontra na literatura (ver por exemplo [24], [50]).

Seja $\mathbb{S}_{l}^{2}$ o hemisfério de raio $l$ em $\mathbb{R}^{3}$

$$
\mathbb{S}_{l}^{2}=\left\{\begin{array}{l}
x_{1}=l \operatorname{sen} \theta_{2} \cos \theta_{1} \\
x_{2}=l \operatorname{sen} \theta_{2} \operatorname{sen} \theta_{1} \\
x_{3}=l \cos \theta_{2}
\end{array}\right.
$$

$\operatorname{com} \theta_{1} \in[0,2 \pi], \theta_{2} \in\left[0, \frac{\pi}{2}\right]$. A métrica induzida é

$$
g=l^{2} d \theta_{2} \otimes d \theta_{2}+l^{2} \operatorname{sen}^{2} \theta_{2} d \theta_{1} \otimes d \theta_{1} .
$$

Por meio da decomposição de Hodge, precisamos apenas do Laplaciano sobre as funções, que tem a forma a seguir:

$$
\Delta^{(0)}=-\left(\frac{1}{l^{2} \operatorname{sen}^{2} \theta_{2}} \partial_{\theta_{1}}^{2}+\frac{\cos \theta_{2}}{l^{2} \operatorname{sen} \theta_{2}} \partial_{\theta_{2}}+\frac{1}{l^{2}} \partial_{\theta_{2}}^{2}\right) .
$$

As condições de contorno seguem aplicando as equações (2.6) e (2.7). Para condições de contorno absoluta temos

$$
\begin{aligned}
0-\text { forms : } & \partial_{\theta_{2}} f\left(\theta_{1}, \pi / 2\right)=0, \\
\text { absoluta } 1-\text { forms : } \quad & \left\{\begin{array}{l}
f_{\theta_{2}}\left(\theta_{1}, \frac{\pi}{2}\right)=0, \\
\partial_{\theta_{2}} f_{\theta_{1}}\left(\theta_{1}, \frac{\pi}{2}\right)=0,
\end{array}\right. \\
2-\text { forms : } & f_{\theta_{1} \theta_{2}}\left(\theta_{1}, \pi / 2\right)=0 .
\end{aligned}
$$

Para as condições de contorno relativa temos

$$
\begin{aligned}
0-\text { forms : } & f\left(\theta_{1}, \pi / 2\right)=0, \\
\text { relativa } \quad 1-\text { forms : } & \left\{\begin{array}{l}
f_{\theta_{1}}\left(\theta_{1}, \frac{\pi}{2}\right)=0, \\
\partial_{\theta_{2}} f_{\theta_{2}}\left(\theta_{1}, \frac{\pi}{2}\right)=0,
\end{array}\right. \\
2-\text { forms : } & \partial_{\theta_{2}} f_{\theta_{1} \theta_{2}}\left(\theta_{1}, \pi / 2\right)=0 .
\end{aligned}
$$


Para obter uma resolução espectral para o Laplaciano sobre as funções em $\mathbb{S}_{1}^{m}$, procederemos como segue: É bem conhecido que uma resolução espectral para o Laplaciano sobre as funções em $\mathbb{S}_{l}^{m}$ pode ser obtida por meio dos polinômios harmônicos. Mais precisamente, denote por $\mathcal{H}_{j}^{m}$ o espaço vetorial dos polinômios $P(x) \in \mathbb{C}\left[x_{0}, \ldots, x_{m}\right]$ que são homogêneos de grau $j$ e harmônicos com respeito ao Laplaciano

$$
\Delta=-\partial_{x_{0}}^{2}-\cdots-\partial_{x_{m}}^{2}
$$

em $\mathbb{R}^{m+1}$. Então, o subespaço $\mathcal{H}=\sum_{n=0}^{\infty} \mathcal{H}_{n}^{m}$ é denso em $C^{\infty}\left(S_{1}^{m}\right)$, pelo teorema de Stone Weierstrass, e portanto $\overline{\mathcal{H}}=L^{2}\left(S_{1}^{m}\right)$. Mais ainda, considerando coordenadas polares $(r, \theta)$,

$$
\Delta=-\partial_{r}^{2}-\frac{m}{r} \partial_{r}+\frac{1}{r^{2}} \Delta_{S_{1}^{m}}
$$

temos

$$
\Delta_{S_{1}^{m}} P(\theta)=n(n+m-1) P(\theta) .
$$

Isto mostra que $L^{2}\left(S_{1}^{m}\right)=\sum_{n=0}^{\infty} \mathcal{H}_{n}^{m}$ e que os autovalores relativos ao autoespaço $\mathcal{H}_{n}^{m}$ são $\lambda_{n}=n(n+m-1)$. É conhecido ainda que

$$
\operatorname{dim} \mathcal{H}_{n}^{m}=\left(\begin{array}{c}
m+n \\
m
\end{array}\right)-\left(\begin{array}{c}
m+n-2 \\
m
\end{array}\right) .
$$

Agora, denote por $S$ a reflexão em $\mathbb{R}^{m+1}$ obtida por trocar o sinal na última coordenada. Então $S$ define uma graduação $\mathbb{Z}_{2}$ em $\mathcal{H}$. Fazendo $P_{ \pm}=P \pm S P$, para $\mathcal{P} \in \mathcal{H}$, é claro que $P=\frac{1}{2}\left(P_{+}+P_{-}\right)$, para cada $P$, e que $S P_{ \pm}= \pm P_{ \pm}$.

Com esta notação e lembrando as equações (3.1) e (3.2) para as condições de contorno, vemos que uma resolução espectral para $\Delta_{\mathbb{S}_{l}^{m}}$ é dada pela família de autoespaços e autovalores com as condições de contorno abaixo

$$
\begin{array}{ll}
\text { absoluta } & \left\{\lambda_{n}=\frac{n(n+m-1)}{l^{2}},\left(\mathcal{H}_{n}^{m}\right)_{+}\right\}, \\
\text {relativa } & \left\{\lambda_{n}=\frac{n(n+m-1)}{l^{2}},\left(\mathcal{H}_{n}^{m}\right)_{-}\right\} .
\end{array}
$$

Precisamos da dimensão destes autoespaços. Consideramos primeiro o caso em que $m=2$. Então $\operatorname{dim} \mathcal{H}_{n}^{2}=2 n+1$. Como obviamente $\left(\mathcal{H}_{0}^{2}\right)_{+}$possue as aplicações constantes, a única partição possível é

$$
\operatorname{dim}\left(\mathcal{H}_{n}^{2}\right)_{+}=n+1 \text { e } \operatorname{dim}\left(\mathcal{H}_{n}^{2}\right)_{-}=n .
$$

Isto ainda pode ser provado por indução, como segue. Assuma que temos $\operatorname{dim}\left(\mathcal{H}_{n}^{2}\right)_{+}=$ $n+1$ e $\operatorname{dim}\left(\mathcal{H}_{n}^{2}\right)_{-}=n$. Seja $P$ um dos $n+1$ geradores de $\left(\mathcal{H}_{n}^{2}\right)_{+}$. Seja $\int_{z}$ denotando o operador inverso de $\partial_{z}$, ou seja, $\int_{z} \partial_{z} P=P$, onde $z$ é a última coordenada na qual fizemos a reflexão. Então $Q=\int_{z} P \in\left(\mathcal{H}_{n+1}^{2}\right)_{-}$, pois $Q$ é obviamente homogêneo de grau $n+1$ e ímpar, por definição. Mais ainda, $\Delta Q=\Delta \int_{z} P=\int_{z} \Delta P=0$, desde que $P$ seja harmônico. Logo $\operatorname{dim}\left(\mathcal{H}_{n+1}^{2}\right)_{-}=n+1$ e então $\operatorname{dim}\left(\mathcal{H}_{n+1}^{2}\right)_{+}=\operatorname{dim} \mathcal{H}_{n+1}^{2}-\operatorname{dim}\left(\mathcal{H}_{n+1}^{2}\right)_{-}=2 n+3-n-1=n+2$.

Usando propriedades dos polinômios homogêneos harmônicos, podemos provar o resultado a seguir para qualquer $m$, que é algo de interesse independente e não encontramos na literatura. 
Proposição 9. O espectro do Laplaciano sobre o hemisfério $m$-dimensional de raio $l$ com condições de contorno de Dirichlet no bordo é $\left\{\lambda_{n}=\frac{n(n+m-1)}{l^{2}}\right\}_{n=0}^{\infty}$, e o autovalor $\lambda_{n}$ tem multiplicidade $\left(\begin{array}{c}m+n-2 \\ m-1\end{array}\right)$. O espectro com condiçôes de contorno de Newmann é o mesmo, mas a multiplicidade é $\left(\begin{array}{c}m+n-1 \\ m-1\end{array}\right)$.

Demonstração. Seja $\mathcal{P}_{n}^{m}$ denotando o conjunto dos polinômios homogêneos de grau $n$ em $\mathbb{R}^{m+1}$. Sabemos que para cada $P \in \mathcal{P}_{n}^{m}$, temos

$$
P\left(x_{0}, \ldots, x_{m}\right)=\sum_{j=0}^{n} Q_{n-j}\left(x_{0}, \ldots, x_{m-1}\right) x_{m}^{j},
$$

com $Q_{l} \in \mathcal{P}_{l}^{m-1}$. Mais ainda, se $P \in \mathcal{H}_{n}^{m}$, então $P$ é completamente determinado por $Q_{n}$ e $Q_{n-1}$. Segue que, se $P \in\left(\mathcal{H}_{n}^{m}\right)_{+}$, então $P$ é completamente determinado por $Q_{n}$ e, portanto,

$$
\operatorname{dim}\left(\mathcal{H}_{n}^{m}\right)_{+}=\operatorname{dim} \mathcal{P}_{n}^{m-1}=\left(\begin{array}{c}
m+n-1 \\
m-1
\end{array}\right)
$$

Lembrando a dimensão de $\mathcal{H}_{n}^{m}$ dada na equação (3.3), concluímos a prova.

Observamos ainda que este resultado, no caso em que $m=2$, pode ser provado por uma analise explícita dos autovalores da equação diferencial $\Delta f=\lambda f$. Decompondo sobre a resolução espectral da restrição do Laplaciano no bordo $\left\{m^{2}, \mathrm{e}^{i m \theta_{1}}\right\}_{m \in \mathbb{Z}}$, a equação dos autovalores decompõe-se na soma da equação diferencial a seguir

$$
\left(\partial_{\theta_{2}}^{2}+\frac{\cos \theta_{2}}{\operatorname{sen} \theta_{2}} \partial_{\theta_{2}}+l^{2} \lambda-\frac{m^{2}}{\operatorname{sen}^{2} \theta_{2}}\right) g\left(\theta_{2}\right)=0
$$

Com a substituição $x=\cos \theta_{2}$, esta equação se reduz a equação de Legendre

$$
\left(1-x^{2}\right) \frac{d^{2} g(x)}{d x^{2}}-2 x \frac{d g(x)}{d x}+\left(\nu(\nu+1)-\frac{m^{2}}{1-x^{2}}\right) g(x)=0,
$$

$\operatorname{com} \lambda=\frac{\nu(\nu+1)}{l^{2}}$. Diferentes pares de soluções lineares independentes da equação (3.4) podem ser dados em termos de pares das funções de Legendre, dependendo dos valores de $\nu$. Procurando por soluções em $L^{2}([0,1])$, a única solução é $P_{n}^{m}(x)$, com $\nu=n \in \mathbb{N}$, e $|m| \leq n$. Impondo condições de contorno absoluta dada pela equação (2.6), obtemos

$$
\frac{2^{m+1}}{\sqrt{\pi}} \operatorname{sen}\left(\frac{n+m}{2} \pi\right) \frac{\Gamma\left(\frac{n+m}{2}+1\right)}{\Gamma\left(\frac{n-m+1}{2}\right)}=0,
$$

com soluções $n+m=2 k \geq 0$. Isto significa que, neste caso, $\lambda=\lambda_{n}=\frac{n(n+1)}{l^{2}}$ com multiplicidade $n+1$. De maneira similar, impondo condições de contorno relativa na equação (2.7), obtemos

$$
\frac{2^{m} \sqrt{\pi}}{\Gamma\left(\frac{n-m}{2}+1\right) \Gamma\left(\frac{-n-m+1}{2}\right)}=0
$$


com soluções $n+m=2 k+1 \geq 1$. Logo $\lambda=\lambda_{n}=\frac{n(n+1)}{l^{2}}$, com multiplicidade $n$.

O próximo passo é obter o espectro do Laplaciano sobre as formas. Para isso usaremos a decomposição de Hodge dada no teorema 6. Os casos absoluto e relativo possuem as decomposições a seguir, respectivamente,

$$
\begin{array}{lll}
\Omega_{\mathrm{abs}}^{0}\left(H_{l}^{2}\right)=\mathcal{H}_{\mathrm{abs}}^{0}\left(H_{l}^{2}\right) \oplus d^{\dagger}\left(\Omega_{\mathrm{abs}}^{1}\left(H_{l}^{2}\right)\right), & & \Omega_{\mathrm{rel}}^{0}\left(H_{l}^{2}\right)=d^{\dagger}\left(\Omega_{\mathrm{rel}}^{1}\left(H_{l}^{2}\right)\right), \\
\Omega_{\mathrm{abs}}^{1}\left(H_{l}^{2}\right)=d\left(\Omega_{\mathrm{abs}}^{0}\left(H_{l}^{2}\right)\right) \oplus d^{\dagger}\left(\Omega_{\mathrm{abs}}^{2}\left(H_{l}^{2}\right)\right), & & \Omega_{\mathrm{rel}}^{1}\left(H_{l}^{2}\right)=d\left(\Omega_{\mathrm{rel}}^{0}\left(H_{l}^{2}\right)\right) \oplus d^{\dagger}\left(\Omega_{\mathrm{rel}}^{2}\left(H_{l}^{2}\right)\right), \\
\Omega_{\mathrm{abs}}^{2}\left(H_{l}^{2}\right)=d\left(\Omega_{\mathrm{abs}}^{1}\left(H_{l}^{2}\right)\right), & & \Omega_{\mathrm{rel}}^{2}\left(H_{l}^{2}\right)=\mathcal{H}_{\mathrm{rel}}^{2}\left(H_{l}^{2}\right) \oplus d\left(\Omega_{\mathrm{rel}}^{1}\left(H_{l}^{2}\right)\right) .
\end{array}
$$

Usando os isomorfismos

$$
*: \Omega_{\mathrm{abs}}^{0}\left(H_{l}^{2}\right) \rightarrow \Omega_{\mathrm{rel}}^{2}\left(H_{l}^{2}\right), *: \Omega_{\mathrm{rel}}^{0}\left(H_{l}^{2}\right) \rightarrow \Omega_{\mathrm{abs}}^{2}\left(H_{l}^{2}\right)
$$

e o fato que, para qualquer autofunção $f, \operatorname{com} \Delta f=\lambda f$,

$$
\Delta^{(1)} d f=\lambda d f, \quad \Delta^{(1)} d^{\dagger}(* f)=\lambda d^{\dagger}(* f), \quad \Delta^{(2)}(* f)=\lambda(* f),
$$

facilmente obtemos que os autovalores de $\Delta^{(q)}, \operatorname{com} q=1,2$, são os mesmos de $\Delta^{(0)}$. E a multiplicidade é dada como segue na tabela abaixo,

\begin{tabular}{|c|c|c|}
\hline Condição de contorno & forma & Multiplicidade \\
\hline \hline \multirow{3}{*}{ Absoluta } & 0 -forma & $n+1$ \\
\cline { 2 - 3 } & 1-forma & $n+1(d)$ e $n\left(d^{\dagger}\right)$ \\
\cline { 2 - 3 } & 2-forma & $n$ \\
\hline \multirow{2}{*}{ Relativa } & 0 -forma & $n$ \\
\cline { 2 - 3 } & 1-forma & $n(d)$ e $n+1\left(d^{\dagger}\right)$ \\
\cline { 2 - 3 } & 2-forma & $n+1$ \\
\hline
\end{tabular}

Podemos então calcular a Torção Analítica. Defina a função

$$
t(s)=\frac{1}{2} \sum_{q=0}^{2}(-1)^{q} q \zeta\left(s, \Delta^{(q)}\right)
$$

tal que $\log T\left(\left(\mathbb{S}_{l}^{2}, g\right), \rho\right)=t^{\prime}(0)$, seguindo a definição (2.11). Então,

$$
t_{\mathrm{abs}}(s)=-t_{\mathrm{rel}}(s)=-\frac{l^{2 s}}{2} \sum_{n=1}^{\infty} \frac{1}{(n(n+1))^{s}} .
$$

Calculamos $t_{\text {abs }}^{\prime}(0)$ como segue. Seja $t_{\text {abs }}(s)=-\frac{l^{2 s}}{2} v(s), \operatorname{com} v(s)=\sum_{n=1}^{\infty}(n(n+1))^{-s}$. Então,

$$
v(s)=4^{s} \sum_{n=2}^{\infty}\left(n^{2}-1\right)^{-s}-\sum_{n=1}^{\infty}\left(n^{2}-\frac{1}{4}\right)^{-s}=4^{s} \zeta(s, \mathrm{i})-z(s, \mathrm{i} / 2),
$$


onde

$$
z(s, a)=\sum_{n=1}^{\infty}\left(n^{2}+a^{2}\right)^{-s}, \quad \zeta(s, a)=\sum_{n=2}^{\infty}\left(n^{2}+a^{2}\right)^{-s}=z(s, a)-\left(1+a^{2}\right)^{-s},
$$

$\mathrm{e} \mathrm{i}=\sqrt{-1}$.

A função $z\left(s, a^{2}\right)=\sum_{n=1}^{\infty}\left(n^{2}+a^{2}\right)^{-s}$ foi estudada em [43], seção 3.1. Temos

$$
z(0, a)=-\frac{1}{2}, \quad z^{\prime}(0, a)=-\log \frac{2 \operatorname{senh} \pi a}{a} .
$$

Logo,

$$
\zeta(0, a)=-\frac{3}{2}, \quad \zeta^{\prime}(0, \mathrm{i})=-\log \pi
$$

o que nos fornece

$$
v^{\prime}(0)=\zeta(0, \mathrm{i}) \log 4+\zeta^{\prime}(0, \mathrm{i})-z^{\prime}(0, \mathrm{i} / 2)=-\log 2 \pi,
$$

e assim

$$
\log T_{\mathrm{abs}}\left(\left(\mathbb{S}_{l}^{2}, g\right), \rho\right)=t_{\mathrm{abs}}^{\prime}(0)=-\frac{1}{2}\left(2 v(0) \log l+v^{\prime}(0)\right)=\frac{1}{2} \log 2 \pi l^{2},
$$

de acordo com o corolário da proposição 8 . 
3. Torção Analítica de variedades com bordo totalmente geodésico 


\section{Capítulo 4}

\section{O disco $m$-dimensional}

Estudaremos a Torção de Reidemeister e a Torção Analítica do disco $m$-dimensional no espaço euclidiano de dimensão $m$. Provamos que a Torção de Reidemeister coincide com uma potência do volume do disco. Estudamos ainda os termos adicionais que surgem na Torção Analítica devido ao bordo, usando as generalizações do teorema de Cheeger Müller, mais precisamente usaremos o teorema 10, Alguns destes resultados estendem para o caso do cone sobre esferas, em particular calculamos diretamente a Torção Analítica do cone sobre o círculo e sobre a esfera de dimensão 2. Comparamos os resultados obtidos através do cálculo direto da Torção Analítica em casos de dimensão baixa, com a fórmula obtida com o teorema 10. Por fim, consideramos a fórmula, que é diferente da que está no teorema 10 para o termo anômalo do bordo, dada por Dai e Fang [13], e mostramos que o resultado obtido usando [13] é inconsistente com o cálculo direto da Torção Analítica do disco de dimensão três e quatro (seção 5.1).

\subsection{Torção de Reidemeister do cone sobre uma esfera}

Seja $D_{l}^{m}=\left\{x \in \mathbb{R}^{m}|| x \mid \leq l\right\}$ o disco de raio $l>0$ no espaço euclidiano $\mathbb{R}^{m}$ com a métrica padrão $g_{E}$ induzida pela imersão. Nesta seção calcularemos a Torção de Reidemeister do disco $m$-dimensional $D_{l}^{m}$. No entanto, consideraremos o caso um pouco mais geral do cone. Considere o cone de ângulo $\alpha, C_{\alpha} S_{l \operatorname{sen} \alpha}^{n}$, construído em $\mathbb{R}^{n+2}$ sobre a esfera $S_{l \operatorname{sen} \alpha}^{n}$, onde $m=n+1$. Note que $C_{\alpha} S_{l \text { sen } \alpha}^{n}$ é exatamente o disco $D_{l}^{m}$ se, e somente se, $\alpha=\frac{\pi}{2}$. Nos outros casos, $C_{\alpha} S_{l \operatorname{sen} \alpha}^{n}$ se quer é uma variedade Riemanniana, mas um espaço com singularidade do tipo cônico (como na definição 11). Mais precisamente, $C_{\alpha} S_{l \operatorname{sen} \alpha}^{n}$ coincide com o cone métrico limitado sobre a espera de raio sen $\alpha$. O espaço obtido removendo o vértice do cone é uma variedade Riemanniana aberta com a métrica induzida pela imersão. Para podermos trabalhar com a Torção de Reidemeister precisamos de algum cuidado já que não é conhecida uma extensão para a teoria de de Rham para este caso. Mais precisamente, não sabemos se 
temos a aplicação de de Rham $\mathcal{A}^{q}$ para espaços com singularidades do tipo cônico em geral. No entanto, procederemos considerando o complexo de cadeias para o espaço total do cone e na hora da escolha da base para as harmônicas consideraremos o cone sem o vértice e as formas estando em $L^{2}$. Este processo é uma maneira formal para a derterminação da Torção de Reidemeister de $C_{\alpha} S_{l \operatorname{sen} \alpha}^{n}$, mas quando $\alpha=\frac{\pi}{2}$ este é o método do cálculo da Torção de Reidemeister para $D_{l}^{m}=C_{\frac{\pi}{2}} S_{l}^{n}$.

Seja $S_{b}^{n}$ a esfera de raio $b>0$ em $\mathbb{R}^{n+1}, S_{b}^{n}=\left\{x \in \mathbb{R}^{n+1}|| x \mid=b\right\}$ (escreveremos simplesmente $S^{n}$ para $\left.S_{1}^{n}\right)$. Seja $C_{\alpha} S_{l \operatorname{sen} \alpha}^{n}$ o cone de ângulo $\alpha$ sobre $S_{l \operatorname{sen} \alpha}^{n}$ em $\mathbb{R}^{n+2}$. Note que o caso do disco corresponde a $D_{l}^{n+1}=C_{\frac{\pi}{2}} S_{l}^{n}$, como mencionado anteriormente. Parametrizamos $C_{\alpha} S_{l \text { sen } \alpha}^{n}$ por

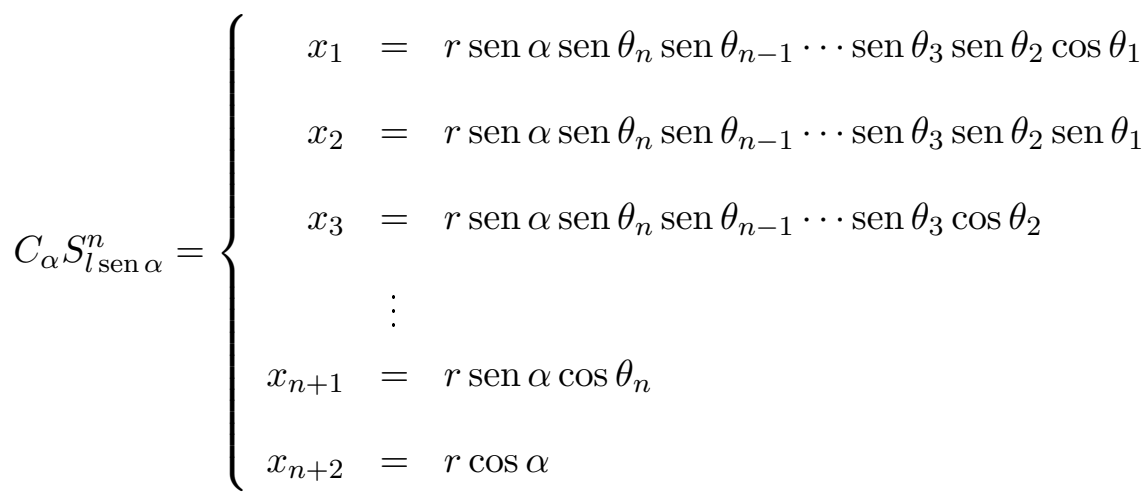

com $r \in[0, l], \theta_{1} \in[0,2 \pi], \theta_{2}, \ldots, \theta_{n} \in[0, \pi], \alpha$ é um numero real positivo fixado e $0<a=$ $\frac{1}{\nu}=\operatorname{sen} \alpha \leq 1$. A métrica induzida é $(r>0)$

$$
\begin{aligned}
g_{E} & =d r \otimes d r+r^{2} g_{S_{a^{2}}^{n}} \\
& =d r \otimes d r+r^{2} a^{2}\left(\sum_{i=1}^{n-1}\left(\prod_{j=i+1}^{n} \operatorname{sen}^{2} \theta_{j}\right) d \theta_{i} \otimes d \theta_{i}+d \theta_{n} \otimes d \theta_{n}\right),
\end{aligned}
$$

$\mathrm{e} \sqrt{\left|\operatorname{det} g_{E}\right|}=(r \operatorname{sen} \alpha)^{n}\left(\operatorname{sen} \theta_{n}\right)^{n-1}\left(\operatorname{sen} \theta_{n-1}\right)^{n-2} \cdots\left(\operatorname{sen} \theta_{3}\right)^{2}\left(\operatorname{sen} \theta_{2}\right)$.

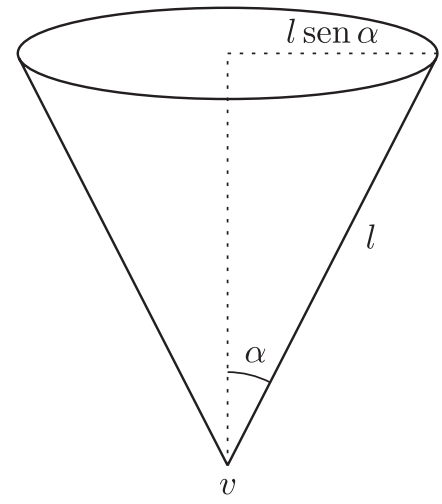

Figura 4.1: Exemplo de $C_{\alpha} S_{l \operatorname{sen} \alpha}^{n}$, quando $n=1$. 
Seja $K$ uma decomposição celular de $C_{\alpha} S_{l \operatorname{sen} \alpha}^{n}$, com uma célula de dimensão máxima, uma $n$-célula e uma 0-célula, $K=c_{n+1}^{1} \cup c_{n}^{1} \cup c_{0}^{1}$. Seja $L$ o subcomplexo de $K$ que é a decomposição celular de $S_{l \operatorname{sen} \alpha}^{n}, L=c_{n}^{1} \cup c_{0}^{1}$. Observamos que, como $\pi_{1}\left(C_{\alpha} S_{l \operatorname{sen} \alpha}^{n}\right)$ é trivial a representação considerada é trivial, o que altera apenas a dimensão do espaço da representação da mesma forma que nas meia-esferas.

Consideraremos primeiro o caso com condições de contorno relativa no bordo.

Proposição 10. $\tau\left(C_{\alpha} S_{l \operatorname{sen} \alpha}^{n}, S_{l \operatorname{sen} \alpha}^{n}\right)=\left({\sqrt{\operatorname{Vol}_{g_{E}}\left(C_{\alpha} S_{l \operatorname{sen} \alpha}^{n}\right)}}^{(-1)^{n} \operatorname{rk}(\rho)}\right.$.

Demonstração. O complexo de espaços vetoriais reais da equação (2.1) fica

$$
C_{\mathrm{rel}}: 0 \longrightarrow \mathbb{R}\left[c_{n+1}^{1}\right] \longrightarrow 0 \longrightarrow 0 \longrightarrow 0 \longrightarrow 0
$$

com base preferida $\mathbf{c}_{n+1}=\left\{c_{n+1}^{1}\right\}$. Para fixar uma base para a homologia, precisamos de uma base graduada ortonormal $\mathbf{a}_{\text {rel }}$ para as formas harmônicas. Para $\Omega^{n+1}\left(C_{\alpha} S_{l \operatorname{sen} \alpha}^{n}\right)$ temos que $\left\{\sqrt{\left|\operatorname{det} g_{E}\right|} d r \wedge d \theta_{1} \wedge \cdots \wedge d \theta_{n}\right\}$ é uma base para as formas harmônicas de dimensão $n+1$, consideremos $\mathbf{h}_{n+1}=\left\{\frac{\sqrt{\left|\operatorname{det} g E_{E}\right|} d r \wedge d \theta_{1} \wedge \cdots \wedge d \theta_{n}}{\sqrt{\operatorname{Vol}_{g_{E}}\left(C_{\alpha} S_{l \operatorname{sen} \alpha}^{n}\right)}}\right\}$. Aplicando a fórmula da equação (2.8) obtemos que $\mathbf{a}_{\text {rel }}=\left\{a_{n+1}^{1}\right\}$, onde

$$
\begin{aligned}
a_{n+1}^{1} & =\mathcal{A}_{n+1}^{\mathrm{rel}}\left(h_{n+1}^{1}\right)=\frac{1}{\sqrt{\operatorname{Vol}_{g_{E}}\left(C_{\alpha} S_{l \operatorname{sen} \alpha}^{n}\right)}} \int_{\mathrm{pt}} * \sqrt{\left|\operatorname{det} g_{E}\right|} d r \wedge d \theta_{1} \wedge \cdots \wedge d \theta_{n} c_{n+1}^{1} \\
& =\frac{1}{\sqrt{\operatorname{Vol}_{g_{E}}\left(C_{\alpha} S_{l \operatorname{sen} \alpha}^{n}\right)}} c_{n+1}^{1} .
\end{aligned}
$$

Como $\mathbf{b}_{q}=\emptyset$, para todo $q$, temos

$$
\left|\operatorname{det}\left(\mathbf{a}_{\mathrm{rel}, n+1} / \mathbf{c}_{n+1}\right)\right|=\frac{1}{\sqrt{\operatorname{Vol}_{g_{E}}\left(C_{\alpha} S_{l \operatorname{sen} \alpha}^{n}\right)}}, \quad\left|\operatorname{det}\left(\mathbf{b}_{q} / \mathbf{c}_{q}\right)\right|=1,0 \leq q \leq n .
$$

Aplicando a definição na equação (2.2), prova-se o resultado.

Considerando $\alpha=\frac{\pi}{2}$, anteriormente, temos o teorema.

Teorema 12. A Torção de Reidemeister do disco $D_{l}^{m}$ de raio $l>0$ em $\mathbb{R}^{m}$ com a métrica $g_{E}$ induzida pela imersão no espaço euclidiano é:

$$
\tau\left(D_{l}^{m}, S_{l}^{m-1}\right)=\left(\sqrt{\operatorname{Vol}_{g_{E}}\left(D_{l}^{m}\right)}\right)^{(-1)^{m-1} \mathrm{rk}(\rho)} .
$$

Consideraremos agora condições de contorno absoluta no bordo.

Proposição 11. $\tau\left(C_{\alpha} S_{l \operatorname{sen} \alpha}^{n}\right)=\left({\sqrt{\operatorname{Vol}_{g_{E}}\left(C_{\alpha} S_{l \operatorname{sen} \alpha}^{n}\right)}}^{\mathrm{rk}(\rho)}\right.$.

Demonstração. O complexo relevante é

$$
C_{\text {abs }}: 0 \longrightarrow \mathbb{R}\left[c_{n+1}^{1}\right] \longrightarrow \mathbb{R}\left[c_{n}^{1}\right] \longrightarrow 0 \longrightarrow 0 \longrightarrow 0
$$


com bases preferidas $\mathbf{c}_{n+1}=\left\{c_{n+1}^{1}\right\}, \mathbf{c}_{n}=\left\{c_{n}^{1}\right\}$ e $\mathbf{c}_{0}=\left\{c_{0}^{1}\right\}$. Assim, $H_{p}(K)=0$, para $p>1$, e $H_{0}(K)=\mathbb{R}\left[c_{0}^{1}\right]$. Uma base para $\mathcal{H}^{0}\left(C_{\alpha} S_{l \operatorname{sen} \alpha}^{n}\right)$ é a forma constante $\{1\}$, temos $\mathbf{h}_{0}=\left\{\frac{1}{\sqrt{\operatorname{Vol}_{g_{E}}\left(C_{\alpha} S_{l \operatorname{sen} \alpha}^{n}\right)}}\right\}$. Aplicando a fórmula da equação (2.8) obtemos $\mathbf{a}_{\mathrm{abs}}=\left\{a_{0}^{1}\right\}$, com

$$
\begin{aligned}
a_{0}^{1} & =\mathcal{A}_{0}^{\mathrm{abs}}\left(h_{0}^{1}\right)=\frac{1}{\sqrt{\operatorname{Vol}_{g_{E}}\left(C_{\alpha} S_{l \operatorname{sen} \alpha}^{n}\right)}} \int_{C_{\alpha} S_{l \operatorname{sen} \alpha}^{n}} * 1 c_{0}^{1} \\
& =\sqrt{\operatorname{Vol}_{g_{E}}\left(C_{\alpha} S_{l \operatorname{sen} \alpha}^{n}\right)} c_{0}^{1} .
\end{aligned}
$$

Como $\mathbf{b}_{q}=\emptyset$ para $q=0, \ldots, n, \tilde{b}_{n+1}^{1}=c_{n+1}^{1}$ e $\partial\left(\tilde{b}_{n+1}^{1}\right)=c_{n}$, temos

$$
\begin{aligned}
& \operatorname{det}\left(\mathbf{a}_{\mathrm{abs}, 0} / \mathbf{c}_{0}\right) \mid & =\sqrt{\operatorname{Vol}_{g_{E}}\left(C_{\alpha} S_{l \operatorname{sen} \alpha}^{n}\right)}, & \\
\left|\operatorname{det}\left(\mathbf{b}_{n+1} / \mathbf{c}_{n}\right)\right| & =1, & & \operatorname{det}\left(\tilde{\mathbf{b}}_{n+1} / \mathbf{c}_{n+1}\right) \mid=1 .
\end{aligned}
$$

Aplicando a definição na equação (2.2), o resultado segue.

Como antes temos,

Teorema 13. Com as mesmas hipóteses do teorema 12, $\tau\left(D_{l}^{m}\right)=\left({\sqrt{\operatorname{Vol}_{g_{E}}\left(D_{l}^{m}\right)}}^{\mathrm{rk}(\rho)}\right.$.

\subsection{A Torção Analítica do disco $m$-dimensional}

O objetivo desta seção é apresentar a comparação entre a Torção de Reidemeister e a Torção Analítica do disco $m$-dimensional no $\mathbb{R}^{m}$. Para isso usaremos o teorema 10. Observamos que esta comparação depende apenas de termos geométricos devido à geometria próxima ao bordo. Como a singularidade no vértice do cone não afeta a geometria perto do bordo, podemos calcular este termo no caso mais geral para $C_{\alpha} S_{l \operatorname{sen} \alpha}^{n}$ ao menos formalmente, isto será tratado na seção 5.2 .

Procedemos em duas partes. Primeiro apresentamos no lema 5 fórmulas para o termo anômalo do bordo em termos de alguns invariantes geométricos. Isto segue diretamente do teorema 10, e no caso ímpar, o termo anômalo do bordo é escrito em termos da característica de Euler do bordo. O caso par é um pouco mais complicado, e precisaremos da notação e das ferramentas introduzidas na seção 2.4. O segundo passo é o lema 6, onde apresentamos todos os invariantes geométricos necessários para calcular a integral que aparece na fórmula do lema 5 e terminamos o cálculo para o caso par.

Antes de começarmos fixamos a notação que será usada no decorrer da seção. A parametrização do cone e a métrica induzida $g_{E}$ são dadas na seção 4.1. Defina as métricas

$$
\begin{aligned}
g_{1}=g_{E} & =d r \otimes d r+r^{2} g_{S^{n}}, \\
g_{0} & =d r \otimes d r+l^{2} g_{S^{n}} .
\end{aligned}
$$


Lema 5. A comparação entre a Torção Analítica e a Torção de Reidemeister do disco $D_{l}^{m}$, para $m=2 p-1$ de dimensão impar e para $m=2 p$ de dimensão par $(p>0)$, com condições de contorno absoluta são respectivamente:

$$
\begin{aligned}
\log T_{\mathrm{abs}}\left(D_{l}^{2 p-1}\right) & =\log \tau\left(D_{l}^{2 p-1}\right)+\frac{1}{4} \mathrm{rk}(\rho) \chi\left(S_{l}^{2 p-2}, g_{E}\right)\left(\log 2+\frac{1}{2} \sum_{n=1}^{p-1} \frac{1}{n}\right) \\
\log T_{\mathrm{abs}}\left(D_{l}^{2 p}\right) & =\log \tau\left(D_{l}^{2 p}\right)+\frac{1}{2} \operatorname{rk}(\rho) \frac{2^{p-1}}{\sqrt{\pi}(2 p-1) ! !} \sum_{j=1}^{p} \frac{1}{2 j-1} \int_{S_{l}^{2 p-1}} \int^{B} \mathcal{S}_{1}^{2 p-1} .
\end{aligned}
$$

Demonstração. Sejam $\omega_{j}, j=0,1$, a 1-forma conexão de $D_{l}^{m}$ associada à métrica $g_{j}, \Omega_{j}=$ $d \omega_{j}+\omega_{j} \wedge \omega_{j}$ a 2-forma curvatura (como na seção 1.2) e ainda, $\Theta=\Theta^{a}{ }_{b}$ a 2-forma curvatura do bordo $S_{l}^{m-1}$ com a métrica Euclidiana padrão. Considere $e(W, g)$ denotando a classe de Euler de $(W, g)$. Usaremos o teorema 10, Temos

$$
\begin{aligned}
& \mathcal{S}_{j}=\frac{1}{2} \sum_{k=1}^{m-1}\left(i^{*} \omega_{j}-i^{*} \omega_{0}\right)^{r}{ }_{\theta_{k}} \hat{e}^{\theta_{k}}, \\
& \mathcal{R}=\hat{\Theta}=\frac{1}{2} \sum_{k, l=1}^{m-1} \Theta^{\theta_{k}}{ }_{\theta_{l}} \hat{e}^{\theta_{k}} \wedge \hat{e}^{\theta_{l}} .
\end{aligned}
$$

Lembramos que

$$
B\left(\nabla_{1}^{T D_{l}^{m}}\right)=\frac{1}{2} \int_{0}^{1} \int^{B} \mathrm{e}^{-\frac{1}{2} \mathcal{R}-u^{2} \mathcal{S}_{j}^{2}} \sum_{k=1}^{\infty} \frac{1}{\Gamma\left(\frac{k}{2}+1\right)} u^{k-1} \mathcal{S}_{1}^{k} d u
$$

e que $B\left(\nabla_{0}^{T D_{l}^{m}}\right)=0$ como $\mathcal{S}_{0}=0$. Vamos determinar $B\left(\nabla_{1}^{T D_{l}^{m}}\right)$. Para isto, note que

$$
\mathcal{R}=-2 \mathcal{S}_{1}^{2}
$$

usando a equação (2.18) ou fazendo o cálculo direto, já que a curvatura do disco $m$ dimensional em $\mathbb{R}^{m}$ é nula.

Portanto, a equação (4.2) fica

$$
\begin{aligned}
B\left(\nabla_{1}^{T D_{l}^{m}}\right) & =\frac{1}{2} \int_{0}^{1} \int^{B} \mathrm{e}^{\left(1-u^{2}\right) \mathcal{S}_{1}^{2}} \sum_{k=1}^{\infty} \frac{1}{\Gamma\left(\frac{k}{2}+1\right)} u^{k-1} \mathcal{S}_{1}^{k} d u \\
& =\frac{1}{2} \int^{B} \sum_{j=0, k=1}^{\infty} \frac{1}{j ! \Gamma\left(\frac{k}{2}+1\right)} \int_{0}^{1}\left(1-u^{2}\right)^{j} u^{k-1} d u \mathcal{S}_{1}^{k+2 j} \\
& =\frac{1}{2} \sum_{j=0, k=1}^{\infty} \frac{1}{k \Gamma\left(\frac{k}{2}+j+1\right)} \int^{B} \mathcal{S}_{1}^{k+2 j} .
\end{aligned}
$$

Como a integral de Berezin é nula sempre que $k+2 j \neq m-1$, obtemos

$$
B\left(\nabla_{1}^{T D_{l}^{m}}\right)=\frac{1}{2 \Gamma\left(\frac{m+1}{2}\right)} \sum_{j=0}^{\left[\frac{m}{2}-1\right]} \frac{1}{m-2 j-1} \int^{B} \mathcal{S}_{1}^{m-1}
$$


Agora consideraremos o caso em que $m$ é par ou ímpar, independentemente. Primeiro, assuma $m=2 p+1(p \geq 0)$. Então, usando a equação (2.14), a equação (4.3) fica

$$
\begin{aligned}
B\left(\nabla_{1}^{T D_{l}^{2 p+1}}\right) & =\frac{1}{2 p !} \sum_{j=0}^{\left[p-\frac{1}{2}\right]} \frac{1}{2 p-2 j} \int^{B} \mathcal{S}_{1}^{2 p} \\
& =\frac{1}{4} \sum_{n=1}^{p} \frac{1}{n} \int^{B} \mathrm{e}^{-\frac{\hat{\Theta}}{2}} \\
& =\frac{1}{4} \sum_{n=1}^{p} \frac{1}{n} \operatorname{Pf}\left(\frac{\Theta}{2 \pi}\right) \\
& =\frac{1}{4} \sum_{n=1}^{p} \frac{1}{n} e\left(S_{l}^{2 p}, g_{E}\right)
\end{aligned}
$$

onde $e\left(S_{l}^{2 p}, g_{E}\right)$ é classe de Euler de $\left(S_{l}^{2 p}, g_{E}\right)$, e usamos o fato que

$$
e\left(S_{l}^{2 p}, g_{l}\right)=\operatorname{Pf}\left(\frac{\Theta}{2 \pi}\right)=\int^{B} e^{-\frac{\hat{\Theta}}{2}}
$$

Logo,

$$
\begin{aligned}
\log \frac{T_{\mathrm{abs}}\left(D_{l}^{2 p+1}\right)}{\tau\left(D_{l}^{2 p+1}\right)} & =\frac{1}{4} \operatorname{rk}(\rho) \chi\left(S_{l}^{2 p}, g_{E}\right) \log 2+\frac{1}{2} \operatorname{rk}(\rho) \int_{S_{l}^{m-1}} B\left(\nabla_{1}^{T D_{l}^{m}}\right) \\
& =\frac{1}{4} \operatorname{rk}(\rho) \chi\left(S_{l}^{2 p}, g_{E}\right) \log 2+\frac{1}{8} \operatorname{rk}(\rho) \sum_{n=1}^{p} \frac{1}{n} \int_{S_{l}^{2 p}} e\left(S^{2 p}, g_{E}\right) \\
& =\frac{1}{4} \operatorname{rk}(\rho) \chi\left(S_{l}^{2 p}, g_{E}\right)\left(\log 2+\frac{1}{2} \sum_{n=1}^{p} \frac{1}{n}\right)
\end{aligned}
$$

Por fim, assuma $m=2 p(p \geq 1)$. Então, a equação (4.3) fica

$$
\begin{aligned}
B\left(\nabla_{1}^{T D_{l}^{2 p}}\right) & =\frac{1}{2 \Gamma\left(p+\frac{1}{2}\right)} \sum_{j=0}^{p-1} \frac{1}{2 p-2 j-1} \int^{B} \mathcal{S}_{1}^{2 p-1} \\
& =\frac{2^{p}}{2 \sqrt{\pi}(2 p-1) ! !} \sum_{j=0}^{p-1} \frac{1}{2(p-j)-1} \int^{B} \mathcal{S}_{1}^{2 p-1} \\
& =\frac{2^{p-1}}{\sqrt{\pi}(2 p-1) ! !} \sum_{j=1}^{p} \frac{1}{2 j-1} \int^{B} \mathcal{S}_{1}^{2 p-1},
\end{aligned}
$$

o que concluí a prova do lema.

Vamos calcular a integral que aparece na segunda equação do lema 5. Antes definimos:

Definição 26. O duplo fatorial de um impar (par) é definido como sendo o produto de todos os números impares (pares) que o antecedem. Assim

$$
\begin{gathered}
(2 n-1) ! !=(2 n-1)(2 n-3) \ldots 3 \cdot 1, \\
(2 n) ! !=(2 n)(2 n-2) \ldots 4 \cdot 2 .
\end{gathered}
$$


Lema 6. Temos

$$
\frac{2^{p-1}}{\sqrt{\pi}(2 p-1) ! !} \int_{S_{l}^{2 p-1}} \int^{B} \mathcal{S}_{1}^{2 p-1}=1
$$

Demonstração. Primeiro, determinaremos a 1-forma conexão associada a $g_{1}$ e a 1 -forma conexão associada a $g_{0}$. Seguiremos a construção da seção 1.2 .

A base não coordenada e o dual respectivo para $g_{1}$ são:

$$
\begin{array}{rlrl}
e_{r} & =\frac{\partial}{\partial r}, & e^{r} & =d r, \\
e_{\theta_{1}} & =\left(r \prod_{j=2}^{n} \operatorname{sen} \theta_{j}\right)^{-1} \frac{\partial}{\partial \theta_{1}}, & e^{\theta_{1}} & =r \prod_{j=2}^{n} \operatorname{sen} \theta_{j} d \theta_{1}, \\
\vdots & \vdots \\
e_{\theta_{n-1}} & =\left(r \operatorname{sen} \theta_{n}\right)^{-1} \frac{\partial}{\partial \theta_{n-1}} & e^{\theta_{n-1}}, & =r \operatorname{sen} \theta_{n} d \theta_{n-1}, \\
e_{\theta_{n}} & =\frac{1}{r} \frac{\partial}{\partial \theta_{n}}, & e^{\theta_{n}} & =r d \theta_{n} .
\end{array}
$$

Então temos $c_{\alpha \beta}^{\gamma}=-c_{\beta \alpha}^{\gamma}, c_{\alpha \alpha}^{\gamma}=0$, para qualquer $\alpha, \gamma$, e os não nulos são: $c_{\theta_{i} r}{ }^{{ }}{ }{ }^{\gamma}=r^{-1}$, e se $k>i, c_{\theta_{i} \theta_{k}}{ }^{\theta_{i}}=\frac{\cos \theta_{k}}{r \prod_{j=k}^{n} \operatorname{sen} \theta_{j}}$. Com estas informações, os símbolos de Christoffel não nulos são:

$$
\begin{aligned}
\Gamma_{\theta_{i} \theta_{i}}^{r} & =-\frac{1}{r}, \Gamma_{\theta_{i}{ }^{\theta_{i}} r}=\frac{1}{r}, \Gamma_{\theta_{i} \theta_{s}}^{\theta_{i}}=\frac{\cos \theta_{s}}{r \prod_{j=s}^{n} \operatorname{sen} \theta_{j}}, \operatorname{com} s>i \\
\Gamma_{\theta_{s}}^{\theta_{i} \theta_{s}} & =\frac{-\cos \theta_{i}}{r \prod_{j=i}^{n} \operatorname{sen} \theta_{j}}, \operatorname{com} i>s .
\end{aligned}
$$

Desta maneira, as entradas não nulas da 1-forma conexão de $g_{1}$ são

$$
\left(\omega_{1}\right)^{\theta_{i}}{ }_{\theta_{k}}=\frac{\cos \theta_{k}}{r \prod_{j=k}^{n} \operatorname{sen} \theta_{j}} e^{\theta_{i}}, i<k, \quad\left(\omega_{1}\right)^{r}{ }_{\theta_{i}}=-\frac{1}{r} e^{\theta_{i}}
$$

A base não coordenada e o seu dual para a métrica $g_{0}$ tem a seguinte forma

$$
\begin{array}{rlrl}
e_{r} & =\frac{\partial}{\partial r} & e^{r}, & =d r \\
e_{\theta_{1}} & =\left(l \prod_{j=2}^{n} \operatorname{sen} \theta_{j}\right)^{-1} \frac{\partial}{\partial \theta_{1}} & e^{\theta_{1}} & =\left(l \prod_{j=2}^{n} \operatorname{sen} \theta_{j}\right) d \theta_{1} \\
\vdots & \vdots \\
e_{\theta_{n-1}} & =\left(l \operatorname{sen} \theta_{n}\right)^{-1} \frac{\partial}{\partial \theta_{n-1}} & e^{\theta_{n-1}} & =\left(l \operatorname{sen} \theta_{n}\right) d \theta_{n-1} \\
e_{\theta_{n}} & =\frac{1}{l} \frac{\partial}{\partial \theta_{n}} & e^{\theta_{n}} & =l d \theta_{n} .
\end{array}
$$


As constantes de Cartan não nulas são: $c_{\theta_{i} \theta_{k}}{ }^{\theta_{i}}=\frac{\cos \theta_{k}}{l \prod_{j=k}^{n} \operatorname{sen} \theta_{j}}$, com $k>i$. Os símbolos de Christoffel não nulos são:

$$
\Gamma_{\theta_{i}}^{\theta_{i} \theta_{s}}=\frac{\cos \theta_{s}}{l \prod_{j=s}^{n} \operatorname{sen} \theta_{j}} \text {, quando } s>i \text {, e } \Gamma_{\theta_{s}}^{\theta_{i} \theta_{s}}=-\frac{\cos \theta_{i}}{l \prod_{j=i}^{n} \operatorname{sen} \theta_{j}} \text {, quando } i>s \text {. }
$$

As entradas não nulas da 1-forma conexão em relação a métrica $g_{0}$ são

$$
\left(\omega_{0}\right)^{\theta_{i}}{ }_{\theta_{s}}=\frac{\cos \theta_{s}}{l \prod_{j=s}^{n} \operatorname{sen} \theta_{j}} e^{\theta_{i}}, i<s .
$$

Segue que as entradas não nulas de $\omega_{1}-\omega_{0}$ são

$$
\left(\omega_{1}-\omega_{0}\right)^{r} \theta_{i}=-\frac{1}{r} e^{\theta_{i}}=-\prod_{j=i+1}^{n} \operatorname{sen} \theta_{j} d \theta_{i} .
$$

Segundo, determinaremos a 2-forma curvatura $\Theta$ associada a métrica induzida. Como $g_{0}$ é uma métrica produto, $\Theta$ é a restrição de $\Omega_{0}$ ao bordo. Temos $\Omega_{0}=d \omega_{0}+\omega_{0} \wedge \omega_{0}$. Escrevemos $\omega_{0}$ nas bases coordenadas

$$
\begin{array}{ll}
\left(\omega_{0}\right)^{r} \theta_{i} & =0 \\
\left(\omega_{0}\right)^{\theta_{i}}{ }_{\theta_{s}} & =\cos \theta_{s} \prod_{j=i+1}^{s-1} \operatorname{sen} \theta_{j} d \theta_{i}, \quad i<s
\end{array}
$$

e $\operatorname{assim} d \omega_{0}$ é

$$
\begin{aligned}
\left(d \omega_{0}\right)^{r}{ }_{\theta_{i}}= & 0, & i \leq n, \\
\left(d \omega_{0}\right)^{\theta_{i}}= & \prod_{\theta_{k}}^{k} \operatorname{sen} \theta_{j} d \theta_{i} \wedge d \theta_{k} & \\
& -\sum_{s=i+1}^{k-1} \cos \theta_{k} \cos \theta_{s} \prod_{j=i+1, j \neq s}^{k-1} \operatorname{sen} \theta_{j} d \theta_{i} \wedge d \theta_{s}, & i<k
\end{aligned}
$$

e $\omega_{0} \wedge \omega_{0}$ é

$$
\begin{aligned}
\left(\omega_{0} \wedge \omega_{0}\right)^{\alpha}{ }_{\alpha}= & 0 \\
\left(\omega_{0} \wedge \omega_{0}\right)^{r}{ }_{\theta_{i}}= & 0 \\
\left(\omega_{0} \wedge \omega_{0}\right)^{\theta_{i}}{ }_{\theta_{k}}= & \sum_{s=i+1}^{k-1} \cos \theta_{s} \cos \theta_{k} \prod_{j=i+1, j \neq s}^{k-1} \operatorname{sen} \theta_{j} d \theta_{i} \wedge d \theta_{s} \\
& +\prod_{j=i+1}^{k} \operatorname{sen} \theta_{j}\left(\prod_{s=k+1}^{n} \operatorname{sen}^{2} \theta_{s}-1\right) d \theta_{i} \wedge d \theta_{k}, \quad i<k
\end{aligned}
$$


Logo, a 2-forma curvatura $\Omega_{0}$ é

$$
\begin{aligned}
\left(\Omega_{0}\right)^{r} \theta_{i} & =0 \\
\left(\Omega_{0}\right)^{\theta_{i}} & =\prod_{\theta_{k}}^{k} \operatorname{sen} \theta_{j} \prod_{s=k+1}^{n} \operatorname{sen}^{2} \theta_{s} d \theta_{i} \wedge d \theta_{k}=\frac{1}{l^{2}} e^{\theta_{i}} \wedge e^{\theta_{k}}, \quad i<k,
\end{aligned}
$$

e conseqüentemente $\Theta=i^{*} \Omega_{0}$ (onde $i$ denota a inclusão do bordo) é

$$
\Theta_{\theta_{k}}^{\theta_{i}}=\prod_{j=i+1}^{k} \operatorname{sen} \theta_{j} \prod_{s=k+1}^{n} \operatorname{sen}^{2} \theta_{s} d \theta_{i} \wedge d \theta_{k}=\frac{1}{l^{2}} e^{\theta_{i}} \wedge, e^{\theta_{k}} \quad i<k .
$$

Terceiro, lembramos que $\mathcal{S}_{1}^{2}=-\frac{1}{2} \mathcal{R}$, usando (2.18) ou fazendo o cálculo direto. Logo

$$
\int^{B} \mathcal{S}_{1}^{2 p-1}=\frac{(-1)^{p-1}}{2^{p-1}} \int^{B} \mathcal{S}_{1} \mathcal{R}^{p-1}
$$

e usando as definições da equação (4.1)

$$
\begin{gathered}
\int^{B} \mathcal{S}_{1}^{2 p-1}=\frac{(-1)^{p-1}}{2^{2 p-1}} \int^{B}\left(\sum_{k=1}^{2 p-1}\left(\omega_{1}-\omega_{0}\right)^{r}{ }_{\theta_{k}} \hat{e}^{\theta_{k}}\right)\left(\sum_{i, j=1}^{2 p-1}\left(\Omega_{0}\right)^{\theta_{i}}{ }_{\theta_{j}} \hat{e}^{\theta_{i}} \wedge \hat{e}^{\theta_{j}}\right)^{p-1} \\
=\frac{(-1)^{p-1}}{2^{p-1} 2^{p}} c_{B}\left(\sum_{\substack{\sigma \in S_{2 p} \\
\sigma(1)=1}} \operatorname{sgn}(\sigma)\left(\omega_{1}-\omega_{0}\right)_{\sigma(2)}^{1}\left(\Omega_{0}\right)^{\sigma(3)}{ }_{\sigma(4)} \ldots\left(\Omega_{0}\right)^{\sigma(2 p-1)}{ }_{\sigma(2 p)}\right),
\end{gathered}
$$

onde $c_{B}=\frac{(-1)^{p(2 p-1)}}{\pi^{\frac{2 p-1}{2}}}$. Observe que $\left(\omega_{1}-\omega_{0}\right)_{\sigma(2)}^{1}$ é uma 1-forma múltipla de $d \theta_{\sigma(2)-1}$ e $\left(\Omega_{0}\right)^{i}{ }_{j}$ é uma 2-forma múltipla de $d_{\theta_{i-1}} \wedge d_{\theta_{j-1}}$. Podemos trocar todas as 2-formas $d_{\theta_{i-1}} \wedge d_{\theta_{j-1}}$, com $i>j$ em cada termo aparecendo no último termo da equação acima, como a matriz é anti simétrica. Então, podemos ordenar o elemento base de maneira que a forma de dimensão máxima apareca em cada termo. Isto produz um sinal coincidindo $\operatorname{com} \operatorname{sgn}(\sigma)$. Mais ainda, como a matriz da 2-forma curvatura é anti-simétrica, o termo genérico da soma na última linha da equação anterior pode ser escrita na forma

$$
\left[\omega_{1}-\omega_{0}\right]_{\sigma(2)}^{1}\left[\Omega_{0}\right]_{\sigma(4)}^{\sigma(3)} \ldots\left[\Omega_{0}\right]_{\sigma(2 p)}^{\sigma(2 p-1)} d \theta_{1} \wedge \ldots \wedge d \theta_{2 p-1},
$$

onde $[\xi]_{j}^{i}$ denota o coeficiente da forma $(\xi)^{i}{ }_{j}$ e $\sigma \in S_{2 p}$ é tal que $\sigma(1)=1$ e $\sigma(2 s-1)<\sigma(2 s)$ para todo $s$. Provemos que

$$
\left[\omega_{1}-\omega_{0}\right]_{\sigma(2)}^{1}\left[\Omega_{0}\right]_{\sigma(4)}^{\sigma(3)} \ldots\left[\Omega_{0}\right]_{\sigma(2 p)}^{\sigma(2 p-1)}=-\prod_{i=2}^{2 p}\left(\operatorname{sen} \theta_{\sigma(i)}\right)^{\sigma(i)-1},
$$

onde $\operatorname{sen} \theta_{2 p}=1$. A prova é por indução. Se $p=1$ a igualdade vale trivialmente. Suponha que é verdade para $p-1$. Por hipótese, se $\tau \in S_{2 p-2} \operatorname{com} \tau(1)=1$, então

$$
\left[\omega_{1}-\omega_{0}\right]_{\tau(2)}^{1}\left[\Omega_{0}\right]_{\tau(4)}^{\tau(3)} \ldots\left[\Omega_{0}\right]_{\tau(2 p-2)}^{\tau(2 p-3)}=-\prod_{i=2}^{2 p-2}\left(\operatorname{sen} \theta_{\tau(i)}\right)^{\tau(i)-1} .
$$


Considere $\sigma \in S_{2 p} \operatorname{com} \sigma(1)=1$. Existe $k_{0}, k_{1}, k_{2}$ tais que $\sigma\left(k_{0}\right)=2 p-2, \sigma\left(k_{1}\right)=2 p-1$ and $\sigma\left(k_{2}\right)=2 p$. Fatorando $\operatorname{sen} \theta_{\sigma\left(k_{i}\right)}, i=0,1,2$, obtemos

$$
\left[\omega_{1}-\omega_{0}\right]_{\sigma(2)}^{1}\left[\Omega_{0}\right]_{\sigma(4)}^{\sigma(3)} \ldots\left[\Omega_{0}\right]_{\sigma(2 p)}^{\sigma(2 p-1)}=\left(\operatorname{sen} \theta_{2 p-2}\right)^{2 p-3}\left(\operatorname{sen} \theta_{2 p-1}\right)^{2 p-2} \times \text { factor }
$$

onde 'factor' é um produto de $\operatorname{sen} \theta_{j}, j \neq \sigma\left(k_{0}\right), \sigma\left(k_{1}\right), \sigma\left(k_{2}\right)$. Desta maneira podemos reescrever 'factor' indexado por uma permutação de $\tau \in S_{2 p-2}$ tal que a hipótese de indução se aplica. Então,

$$
\left[\omega_{1}-\omega_{0}\right]_{\sigma(2)}^{1}\left[\Omega_{0}\right]_{\sigma(4)}^{\sigma(3)} \ldots\left[\Omega_{0}\right]_{\sigma(2 p)}^{\sigma(2 p-1)}=-\prod_{j=2}^{2 p}\left(\operatorname{sen} \theta_{\sigma(j)}\right)^{\sigma(j)-1} .
$$

Assim, provamos que

$$
\int^{B} \mathcal{S}_{1}^{2 p-1}=c_{B} \frac{(-1)^{p}(2 p-1) !}{2^{p-1} 2^{p}} \prod_{j=2}^{2 p-1}\left(\operatorname{sen} \theta_{j}\right)^{j-1} d \theta_{1} \wedge \ldots \wedge d \theta_{2 p-1} .
$$

Então,

$$
\begin{aligned}
\frac{2^{p-1}}{\sqrt{\pi}(2 p-1) ! !} \int_{S_{l}^{2 p-1}} \int^{B} \mathcal{S}_{1}^{2 p-1} & =c_{B} \frac{2^{p-1}}{\sqrt{\pi}(2 p-1) ! !} \frac{(-1)^{p}(2 p-1) !}{2^{p-1} 2^{p} l^{2 p-1}} \operatorname{Vol}\left(S_{l}^{2 p-1}\right) \\
& =\frac{(2 p-1) ! \sqrt{\pi}}{2^{p-1}(p-1) ! \sqrt{\pi}(2 p-1) ! !}
\end{aligned}
$$

E claramente

$$
\frac{(2 p-1) !}{(p-1) !(2 p-1) ! !}=2^{p-1}
$$

Logo,

$$
\frac{2^{p-1}}{\sqrt{\pi}(2 p-1) ! !} \int_{S_{l}^{2 p-1}} \int^{B} \mathcal{S}_{1}^{2 p-1}=1
$$

Com os lemas 5 e 6 está provado o teorema.

Teorema 14. A Torção Analítica do disco $D_{l}^{m}$ de raio $l>0$ em $\mathbb{R}^{m}$ com a métrica padrão $g_{E}$ induzida pela imersão é $(p>0)$ :

$$
\begin{aligned}
& \log T_{\text {rel }}\left(D_{l}^{2 p-1}\right)=\log T_{\text {abs }}\left(D_{l}^{2 p-1}\right)=\frac{1}{2} \operatorname{rk}(\rho) \log \tau\left(D_{l}^{2 p-1}\right)+\frac{1}{2} \operatorname{rk}(\rho) \log 2+\frac{1}{4} \operatorname{rk}(\rho) \sum_{n=1}^{p-1} \frac{1}{n} \\
& -\log T_{\text {rel }}\left(D_{l}^{2 p}\right)=\log T_{\text {abs }}\left(D_{l}^{2 p}\right)=\frac{1}{2} \operatorname{rk}(\rho) \log \tau\left(D_{l}^{2 p}\right)+\frac{1}{2} \operatorname{rk}(\rho) \sum_{n=1}^{p} \frac{1}{2 n-1}
\end{aligned}
$$

Concluímos esta seção calculando o termo anômalo do bordo usando a fórmula dada no teorema 1 de [13]. No caso par apresentamos uma versão formal para o caso do cone sobre esferas. Precisamos introduzir alguma notação. Seja $W$ uma variedade Riemanniana 
compacta com bordo $\partial W$ de classe $C^{\infty}$. Considere a homotopia $\omega_{t}=\omega_{0}+t\left(\omega_{1}-\omega_{0}\right)$ entre a 1-forma conexão $\omega_{1}$ e a 1 forma conexão $\omega_{0}$, onde $\omega_{1}\left(\omega_{0}\right)$ é a 1-forma conexão associada a $g_{1}\left(g_{0}\right)$ como na seção 2.4. Considere $\Omega_{t}=d \omega_{t}+\omega_{t} \wedge \omega_{t}$ a 2-forma curvatura correspondente. A classe de Chern-Simons associada a classe de Euler de $\Omega_{t}$ será denotada por $\tilde{e}\left(g_{0}, g_{1}\right)$, e satisfaz dẽ $\left(g_{0}, g_{1}\right)=e\left(g_{1}\right)-e\left(g_{0}\right)$, onde $e\left(g_{j}\right)$ é a classe de Euler de $\Omega_{j}$ (para mais informação sobre a classe de Chern-Simons associada a classe de Euler ver [33]). Considere a métrica $g_{1}=g_{E}$ e $g_{0}$ como anteriormente. Usando o teorema 1 de [13] temos as fórmulas:

$$
\begin{aligned}
& \log \frac{T_{\mathrm{abs}}\left(D_{l}^{2 p-1}\right)}{\tau\left(D_{l}^{2 p-1}\right)}=\frac{1}{4} \operatorname{rank}(\rho) \chi\left(S_{l}^{2 p-2}, g_{E}\right) \log 2, \\
& \log \frac{T_{\mathrm{abs}}\left(C_{\alpha} S_{l \operatorname{sen} \alpha}^{2 p-1}\right)}{\tau\left(C_{\alpha} S_{l \operatorname{sen} \alpha}^{2 p-1}\right)}=\frac{1}{2} \operatorname{rank}(\rho) \int_{S_{l \operatorname{sen} \alpha}^{2 p-1}} i^{*} \tilde{e}\left(g_{0}, g_{E}\right),
\end{aligned}
$$

onde $i$ denota a inclusão do bordo. Procedendo da mesma maneira que na prova do lema 6 , calculamos a integral que aparece na equação (4.5). Obtemos

$$
\int_{S_{l \operatorname{sen} \alpha}^{2 p-1}} i^{*} \tilde{e}\left(g_{0}, g_{E}\right)=\frac{(-1)^{p+1}(2 p) ! \operatorname{Vol}\left(S_{l \operatorname{sen} \alpha}^{2 p-1}\right)}{(4 \pi)^{p} l^{2 p-1} p !(\operatorname{sen} \alpha)^{2 p-2}} \sum_{k=0}^{p-1}(-1)^{k} \frac{(\operatorname{sen} \alpha)^{2 k}}{2 k+1}\left(\begin{array}{c}
p-1 \\
k
\end{array}\right) .
$$

Em particular, para o caso do disco $(\operatorname{sen} \alpha=1)$,

$$
\int_{S_{l}^{2 p-1}} i^{*} \tilde{e}\left(g_{0}, g_{E}\right)=(-1)^{p+1}
$$

Comparando a abordagem de [4] com a abordagem de [13], vemos que em [4] o termo do bordo é obtido considerando uma homotopia entre as duas métricas $g_{1}$ e $g_{0}$, uma deformação da métrica $g_{1}$ na métrica $g_{0}$, fazendo a comparação entre a Torção Analítica com estas duas métricas. Em [13], o termo do bordo é obtido considerando uma homotopia entre as 1-forma conexões $\omega_{1}$ e $\omega_{0}$, o que produz um termo "menor" que não capta toda a contribuição do bordo (ao menos no caso de dimensão par). Faremos nas seções 4.3 e 5.1 o cálculo explícito da Torção Analítica de $D^{3}$ e $D^{4}$ confirmando esta última afirmação.

\subsection{Torção Analítica de $C_{\alpha} S_{l \operatorname{sen} \alpha}^{1}$ e $C_{\alpha} S_{l \operatorname{sen} \alpha}^{2}$}

Nesta seção calcularemos a Torção Analítica dos cones $C_{\alpha} S_{l \operatorname{sen} \alpha}^{1}$ e $C_{\alpha} S_{l \operatorname{sen} \alpha}^{2}$ usando a definição dada na equação (2.11). Para isto precisaremos conhecer explicitamente o espectro do Laplaciano das formas desses espaços singulares, e depois uma sutil representação para a extensão analítica da função zeta associada, que permita calcular sua derivada no zero. O primeiro ponto do problema foi originalmente estudado por J. Cheeger em [9] (veja ainda [18], [42] e [45]). No trabalho de Cheeger, a teoria de Hodge-de Rham foi desenvolvida para espaço com singularidades do tipo cônico (ver seção 1.4). Em particular, ele provou que o Laplaciano nas formas é um operador auto-adjunto no espaço da formas quadrado integráveis sobre o 
cone, se algum conjunto apropriado de condições de contorno é assumido no vértice do cone (ver seção 1.4). No entanto, no nosso caso particular do cone sobre as esferas não é necessário condições de contorno no vértice como os grupos de homologia de dimensão média da seção do cone é trivial (ver [8]). Apresentamos o espectro de $\Delta^{(q)}$ sobre $C_{\alpha} S_{l \operatorname{sen} \alpha}^{1}$ e sobre $C_{\alpha} S_{l \operatorname{sen} \alpha}^{2}$ no lema 7 e lema 8 abaixo, respectivamente. Depois calcularemos a derivada das funções zeta, usaremos as idéias da seção 1.5. Em resumo, os autovalores de $\Delta_{C_{\alpha}}^{(q)} S_{l \operatorname{sen} \alpha}^{n}$ podem ser identificados com os zeros de $z_{\nu, k}$ de alguma combinação de funções de Bessel e suas derivadas, sendo enumerados com dois índices positivos como $\lambda_{n, k}^{(q)}=z_{u_{n}, k}^{2}$, onde $u_{n}$ depende dos autovalores do Laplaciano em algum espaço de $q$-formas da seção do cone. Usando estimativas clássicas para os zeros de funções de Bessel é possível provar que as seqüências relevantes $U$ e $S$ estão contidas na classe de seqüências abstratas introduzidas em [43], 46] e definidas na seção 1.5. Isto significa que podemos usar o método descrito nesta seção para calcular a derivada da função zeta associada a $\Delta^{(q)}$.

\subsubsection{Espectro do Laplaciano sobre as formas}

Nesta seção calcularemos o espectro do Laplaciano sobre as formas. A forma geral para soluções da equação dos autovalores é apresentada na seção [1.4 (ver também [9] e [10]). Entretanto, apresentaremos a forma explícita para determinar as soluções neste caso estudado com alguns detalhes do cálculo que não se encontra na literatura. Mais ainda, apresentamos no decorrer das provas, o sistema completo de autoformas do operador Laplaciano. Denotaremos por $\{k: \lambda\}$ o conjunto de autovalores $\lambda$ com multiplicidade $k$.

Lema 7. O espectro do operador Laplaciano $\Delta_{C_{\alpha} S_{l \operatorname{sen} \alpha}^{1}}^{(q)}$ sobre as q-formas com condiçôes de contorno absoluta é (onde $\nu=\operatorname{cosec} \alpha)$ :

$$
\begin{aligned}
\operatorname{Sp} \Delta_{C_{\alpha} S_{l \operatorname{sen} \alpha}^{1}}^{(0)}= & \left\{j_{1, k}^{2} / l^{2}\right\}_{k=1}^{\infty} \cup\left\{2:\left(j_{\nu n, k}^{\prime}\right)^{2} / l^{2}\right\}_{n, k=1}^{\infty}, \\
\operatorname{Sp} \Delta_{C_{\alpha}}^{(1)} S_{l \operatorname{sen} \alpha}^{1}= & \left\{j_{0, k}^{2} / l^{2}\right\}_{k=1}^{\infty} \cup\left\{j_{1, k}^{2} / l^{2}\right\}_{k=1}^{\infty} \cup\left\{2: j_{\nu n, k}^{2} / l^{2}\right\}_{n, k=1}^{\infty} \\
& \cup\left\{2:\left(j_{\nu n, k}^{\prime}\right)^{2} / l^{2}\right\}_{n, k=1}^{\infty}, \\
\operatorname{Sp} \Delta_{C_{\alpha} S_{l \operatorname{sen} \alpha}^{1}}^{(2)}= & \left\{j_{0, k}^{2} / l^{2}\right\}_{k=1}^{\infty} \cup\left\{2: j_{\nu n, k}^{2} / l^{2}\right\}_{n, k=1}^{\infty} .
\end{aligned}
$$

O espectro do operado Laplaciano $\Delta_{C_{\alpha} S_{l \operatorname{sen} \alpha}^{1}}^{(q)} \quad$ sobre as q-formas com condições de contorno relativa é:

$$
\begin{aligned}
\operatorname{Sp} \Delta_{C_{\alpha} S_{l \operatorname{sen} \alpha}^{1}}^{(0)} & \left\{j_{0, k}^{2} / l^{2}\right\}_{k=1}^{\infty} \cup\left\{2: j_{\nu n, k}^{2} / l^{2}\right\}_{n, k=1}^{\infty}, \\
\operatorname{Sp} \Delta_{C_{\alpha} S_{l \operatorname{sen} \alpha}^{(1)}=}^{1}=\left\{j_{0, k}^{2} / l^{2}\right\}_{k=1}^{\infty} \cup\left\{j_{1, k}^{2} / l^{2}\right\}_{k=1}^{\infty} \cup\left\{2: j_{\nu n, k}^{2} / l^{2}\right\}_{n, k=1}^{\infty} & \cup\left\{2:\left(j_{\nu n, k}^{\prime}\right)^{2} / l^{2}\right\}_{n, k=1}^{\infty}, \\
& \operatorname{Sp} \Delta_{C_{\alpha} S_{l \operatorname{sen} \alpha}^{(2)}=}^{(2)}\left\{j_{1, k}^{2} / l^{2}\right\}_{k=1}^{\infty} \cup\left\{2:\left(j_{\nu n, k}^{\prime}\right)^{2} / l^{2}\right\}_{n, k=1}^{\infty} .
\end{aligned}
$$


Demonstração. Parametrizamos $C_{\alpha} S_{l \operatorname{sen} \alpha}^{1}$ por

$$
C_{\alpha} S_{l \operatorname{sen} \alpha}^{1}=\left\{\begin{array}{l}
x_{1}=r \operatorname{sen} \alpha \cos \theta \\
x_{2}=r \operatorname{sen} \alpha \operatorname{sen} \theta \\
x_{3}=r \cos \alpha
\end{array}\right.
$$

onde $(r, \theta) \in[0, l] \times[0,2 \pi], l$ e $\alpha$ são números reais fixados e $0<a=\operatorname{sen} \alpha \leq 1$. A métrica induzida é

$$
g=d r \otimes d r+a^{2} r^{2} d \theta \otimes d \theta
$$

e o operador $*$ de Hodge age nos elementos da base da seguinte forma

$$
\begin{aligned}
& *: 1 \mapsto \operatorname{ardr} \wedge d \theta ; \\
& *: d r \mapsto \operatorname{ard\theta }, \quad *: d \theta \mapsto-\frac{1}{a r} d r ; \\
& *: d r \wedge d \theta \mapsto \frac{1}{a r} .
\end{aligned}
$$

O Laplaciano nas formas é

$$
\begin{aligned}
\Delta^{(0)}(f)= & -\partial_{r}^{2} f-\frac{1}{r} \partial_{r} f-\frac{1}{a^{2} r^{2}} \partial_{\theta}^{2} f \\
\Delta^{(1)}\left(f_{r} d r+f_{\theta} d_{\theta}\right)= & \left(-\partial_{r}^{2} f_{r}-\frac{1}{a^{2} r^{2}} \partial_{\theta}^{2} f_{r}+\frac{1}{r^{2}} f_{r}-\frac{1}{r} \partial_{r} f_{r}+\frac{2}{a^{2} r^{3}} \partial_{\theta} f_{\theta}\right) d r \\
& +\left(-\partial_{r}^{2} f_{\theta}-\frac{1}{a^{2} r^{2}} \partial_{\theta}^{2} f_{\theta}+\frac{1}{r} \partial_{r} f_{\theta}-\frac{2}{r} \partial_{\theta} f_{r}\right) d \theta \\
\Delta^{(2)}(f d r \wedge d \theta)= & -\partial_{r}^{2} f+\frac{1}{r} \partial_{r} f-\frac{2}{r^{2}} f-\frac{1}{a^{2} r^{2}} \partial_{\theta}^{2} f
\end{aligned}
$$

Usando a decomposição descrita na equação (2.5), obtemos a partir das equações (2.6), e (2.7), os conjuntos a seguir de condições de contorno. Para as 0-formas:

$$
\text { rel. : } \quad \omega(l, \theta)=0, \quad \text { abs. : } \quad\left(\partial_{r} \omega\right)(l, \theta)=0,
$$

e as condições de contorno relativa coincide com as condições de contorno de Dirichlet. Para as 2-formas

$$
\text { abs. : } \quad \omega(l, \theta)=0, \quad \text { rel. : } \quad\left(\partial_{r} \frac{\omega}{r}\right)(l, \theta)=0,
$$

e as condições de contorno absoluta coincide com as condições de contorno de Dirichlet. Para 1-formas:

$$
\text { abs. : }\left\{\begin{array} { l } 
{ \omega _ { r } ( l , \theta ) = 0 , } \\
{ ( \partial _ { r } \omega _ { \theta } ) ( l , \theta ) = 0 , }
\end{array} \quad \text { rel. : } \left\{\begin{array}{l}
\omega_{\theta}(l, \theta)=0 \\
\left(\partial_{r}\left(a r \omega_{r}\right)+\frac{1}{a r} \partial_{\theta} \omega_{\theta}\right)(l, \theta)=0
\end{array}\right.\right.
$$

onde $\omega(r, \theta)=\omega_{r}(r, \theta) d r+\omega_{\theta}(r, \theta) d \theta$. 
Agora iremos solucionar as equações dos autovalores. Note que o Laplaciano nas 2-formas coincide com o das 0-formas pela transformada de Liouville $f=r h$. Considere a equação dos autovalores para o Laplaciano sobre as 0-formas

$$
\Delta^{(0)} f=\left(-\partial_{r}^{2}-\frac{1}{r} \partial_{r}-\frac{1}{a^{2} r^{2}} \partial_{\theta}^{2}\right) f=\lambda^{2} f
$$

Podemos decompor o problema sobre o autoespaço de $-\partial_{\theta}^{2}$. De fato, $\phi_{n}(\theta)=\mathrm{e}^{\text {in } \theta}$ é um sistema completo de autofunções para $-\partial_{\theta}^{2}$ sobre o círculo $S^{1}$, e o autovalor de $\phi_{n}$ é $\lambda_{n}=n^{2}$, $n \in \mathbb{Z}$. Então,

$$
\Delta^{(0)}=\sum_{n \in \mathbb{Z}} L_{n} \Pi_{n}
$$

onde $\Pi_{n}$ é a projeção sobre o subespaço gerado pela autofunção $\phi_{n}$ do autoespaço relativo ao autovalor $\lambda_{n} \mathrm{e}$

$$
L_{n}=-\partial_{r}^{2}-\frac{1}{r} \partial_{r}+\frac{\nu^{2} n^{2}}{r^{2}}
$$

onde $\nu=\frac{1}{a}$. Como $\lambda_{n}=\lambda_{-n}, \Pi_{n}=\Pi_{-n}$. Como $-\partial_{\theta}^{2}$ possui um sistema completo

$$
\left\{\lambda_{n}=n^{2} ; \phi_{n,+}(\theta)=\mathrm{e}^{\mathrm{i} n \theta}, \phi_{n,-}(\theta)=\mathrm{e}^{-\mathrm{i} n \theta}\right\}_{n \in \mathbb{N}}
$$

onde todos os autovalores são duplos, a não ser $\lambda_{0}=0$ que é simples, e ainda $L_{n}=L_{-n}$,

$$
\Delta^{(0)}=L_{0} \Pi_{0} \oplus \sum_{n=1}^{\infty} L_{n}\left(\Pi_{n,+} \oplus \Pi_{n,-}\right),
$$

onde $\Pi_{ \pm, n}$ é a projeção sobre o autoespaço gerado por $\phi_{ \pm n}$ no autoespaço de $\lambda_{n}$ (na verdade o autoespaço de $\lambda_{n}$ é gerado pelas duas autofunções $\phi_{ \pm n}$ para todo $n \neq 0$ ). Agora, resolvemos a equação dos autovalores para $L_{n}$ sobre $L^{2}(0, l)$,

$$
L_{n} u=\left(-\partial_{r}^{2}-\frac{1}{r} d_{r}+\frac{\nu^{2} n^{2}}{r^{2}}\right) u=\lambda^{2} u .
$$

Esta equação pode ser resolvida em termos de funções de Bessel. A equação (4.10) possui duas soluções linearmente independente (assuma que $\mu=\nu n$ não é inteiro) $y_{ \pm \mu}(r)=J_{ \pm|\mu|}(\lambda r)$ (onde assumimos que $\lambda>0$ ). Mas $J_{-|\mu|}(r)$ diverge como $r^{-|\mu|}$ em $r=0$ e portanto não satisfaz a condição de contorno em $r=0$, ou não está em $L^{2}(0, l)$ (dependendo do valor de $\mu$ ). Logo consideramos apenas a solução $y_{+}$. Isto significa que a equação dos autovalores (4.10) para $L_{n}$ possui a solução $\psi_{n}(r)=J_{|\nu n|}(\lambda r)$, para cada $n \in \mathbb{Z}$; em particular possui a solução $\psi_{n}(r)=J_{\nu n}(\lambda r)$, se $n \geq 0$, como $\nu \geq 0$. Portanto um sistema linearmente independente de soluções da equação de autovalores (4.9) para $\Delta^{(0)}$ é

$$
\begin{aligned}
& \left\{\phi_{0}(\theta) \psi_{0}(r)=J_{0}(\lambda r)\right\} \\
& \cup\left\{\phi_{n,+}(\theta) \psi_{n}(r)=\mathrm{e}^{\mathrm{i} n \theta} J_{\nu n}(\lambda r), \phi_{n,-}(\theta) \psi_{n}(r)=\mathrm{e}^{-\mathrm{i} n \theta} J_{\nu n}(\lambda r)\right\}_{n \in \mathbb{N}_{0}} .
\end{aligned}
$$


As soluções para $\Delta^{(2)}$ são dadas por meio da inversa da transformada de Liouville acima,

$$
\begin{aligned}
& \left\{\phi_{0}(\theta) \psi_{0}(r)=x J_{0}(\lambda r)\right\} \\
& \cup\left\{\phi_{n,+}(\theta) \psi_{n}(r)=r \mathrm{e}^{\mathrm{i} n \theta} J_{\nu n}(\lambda r), \phi_{n,-}(\theta) \psi_{n}(r)=r \mathrm{e}^{-\mathrm{i} n \theta} J_{\nu n}(\lambda r)\right\}_{n \in \mathbb{N}_{0}} .
\end{aligned}
$$

Note que a transformada de Lioville, neste caso, vem da relação $\Delta *=* \Delta$.

A equação dos autovalores para o Laplaciano sobre as 1-formas é:

$$
\Delta^{(1)} \omega=\lambda^{2} \omega
$$

onde $\omega=f_{r} d r+f_{\theta} d \theta$. Isto corresponde ao sistema de equações diferenciais parciais

$$
\left\{\begin{array}{l}
-\partial_{r}^{2} f_{r}-\frac{1}{r} \partial_{r} f_{r}+\frac{-\nu^{2} \partial_{\theta}^{2}+1}{r^{2}} f_{r}+\frac{2 \nu^{2}}{r^{3}} \partial_{\theta} f_{\theta}=\lambda^{2} f_{r} \\
-\partial_{r}^{2} f_{\theta}+\frac{1}{r} \partial_{r} f_{\theta}+\frac{-\nu^{2} \partial_{\theta}^{2}}{r^{2}} f_{\theta}-\frac{2}{r} \partial_{\theta} f_{r}=\lambda^{2} f_{\theta}
\end{array}\right.
$$

Como uma base para $L^{2}\left(S^{1}\right)$ é dada por meio das funções e $\mathrm{e}^{\mathrm{i} n \theta} \operatorname{com} n \in \mathbb{Z}$, consideramos soluções do tipo $\omega=f_{r}(r) \mathrm{e}^{\mathrm{i} m \theta} d r+f_{\theta}(r) \mathrm{e}^{\mathrm{i} n \theta} d \theta$, com $m, n \in \mathbb{Z}$. Substituindo em (4.14) temos

$$
\left\{\begin{array}{l}
-\partial_{r}^{2} f_{r} \mathrm{e}^{\mathrm{i} m \theta}-\frac{1}{r} \partial_{r} f_{r} \mathrm{e}^{\mathrm{i} m \theta}+\frac{(\nu m)^{2}+1}{r^{2}} f_{r} \mathrm{e}^{\mathrm{i} m \theta}+\frac{2 \mathrm{i} \nu^{2} n}{r^{3}} f_{\theta} \mathrm{e}^{\mathrm{i} n \theta}=\lambda^{2} f_{r} \mathrm{e}^{\mathrm{i} m \theta} \\
-\partial_{r}^{2} f_{\theta} \mathrm{e}^{\mathrm{i} n \theta}+\frac{1}{r} \partial_{r} f_{\theta} \mathrm{e}^{\mathrm{i} n \theta}+\frac{(\nu n)^{2}}{r^{2}} f_{\theta} \mathrm{e}^{\mathrm{i} n \theta}-\frac{2 \mathrm{i} m}{r} f_{r} \mathrm{e}^{\mathrm{i} m \theta}=\lambda^{2} f_{\theta} \mathrm{e}^{\mathrm{i} n \theta}
\end{array}\right.
$$

que é satisfeito se, e somente se, $m=n$. Portanto, segue que as soluções da equação (4.13) são da forma

$$
\omega=\mathrm{e}^{\mathrm{i} n \theta}\left(f_{r}(r) d r+f_{\theta}(r) d \theta\right)
$$

$\operatorname{com} n \in \mathbb{Z}$. Em outras palavras, o operador $\Delta^{(1)}$ decompõe-se como

$$
\Delta^{(1)}=\sum_{n \in \mathbb{Z}} L_{n} \Pi_{n}
$$

onde

$$
L_{n}=\left(\begin{array}{cc}
-\partial_{r}^{2}-\frac{1}{r} \partial_{r}+\frac{(\nu n)^{2}+1}{r^{2}} & \frac{2 \mathrm{i} \nu^{2} n}{r^{3}} \\
-\frac{2 \mathrm{i} n}{r} & -\partial_{r}^{2}+\frac{1}{r} \partial_{r}+\frac{(\nu n)^{2}}{r^{2}}
\end{array}\right),
$$

sobre $\left(L^{2}(0,1)\right)^{2}$, e $\Pi_{n}$ é a projeção sobre o subespaço gerado por e $\mathrm{e}^{i n \theta}$ do autoespaço relativo ao autovalor $n^{2}$ de $-\partial_{\theta}^{2}$. Logo, precisamos resolver a equação dos autovalores

$$
L_{n} u=\lambda_{n}^{2} u
$$

onde $u=\left(f_{r}, f_{\theta}\right)$ são duas funções em $L^{2}(0, l)$. Isto corresponde ao sistema

$$
\left\{\begin{array}{l}
-\partial_{r}^{2} f_{r}-\frac{1}{r} \partial_{r} f_{r}+\frac{(\nu n)^{2}+1}{r^{2}} f_{r}+\frac{2 \mathrm{i} \nu^{2} n}{r^{3}} f_{\theta}=\lambda_{n}^{2} f_{r}, \\
-\partial_{r}^{2} f_{\theta}+\frac{1}{r} \partial_{r} f_{\theta}+\frac{(\nu n)^{2}}{r^{2}} f_{\theta}-\frac{2 \mathrm{i} n}{r} f_{r}=\lambda_{n}^{2} f_{\theta} .
\end{array}\right.
$$


Com a mudança de base $\left(f_{r}, f_{\theta}\right)=\left(\nu g_{r},-\mathrm{i} r g_{\theta}\right)$, obtemos

$$
\left\{\begin{array}{l}
\left(-\partial_{r}^{2}-\frac{1}{r} \partial_{r}+\frac{(\nu n+1)^{2}}{r^{2}}\right)\left(g_{r}+g_{\theta}\right)=\lambda_{n}^{2}\left(g_{r}+g_{\theta}\right), \\
\left(-\partial_{r}^{2}-\frac{1}{r} \partial_{r}+\frac{(\nu n-1)^{2}}{r^{2}}\right)\left(g_{r}-g_{\theta}\right)=\lambda_{n}^{2}\left(g_{r}-g_{\theta}\right) .
\end{array}\right.
$$

Usando resultados sobre soluções de equações de Bessel e considerando apenas soluções $L^{2}$ (da mesma maneira que no caso das 0-formas), temos que um conjunto completo de soluções linearmente independentes é dado pelas duas autofunções

$$
\left\{\begin{array}{l}
\left(g_{r}, g_{\theta}\right)_{n}=\left(J_{|\nu n+1|}\left(\lambda_{n} r\right), J_{|\nu n+1|}\left(\lambda_{n} r\right)\right) \\
\left(g_{r}, g_{\theta}\right)_{n}=\left(J_{|\nu n-1|}\left(\lambda_{n} r\right),-J_{|\nu n-1|}\left(\lambda_{n} r\right)\right) .
\end{array}\right.
$$

Portanto, a equação dos autovalores (4.13) relativa ao operador $\Delta^{(1)}$, possui o sistema completo de soluções linearmente independente $L^{2} \operatorname{com} n \in \mathbb{Z}$

$$
\left\{\omega_{n, \pm}=J_{|\nu n \pm 1|}\left(\lambda_{n} r\right) \mathrm{e}^{\mathrm{i} n \theta}(\nu d r-i r d \theta)\right\} .
$$

Por fim, aplicamos as condições de contorno. Para 0-formas, decompomos como na equação (2.5) $\omega_{t g}=\omega$ e $\omega_{\text {norm }}=0$. As condições de contorno relativa dadas na equação (4.6) aplicadas nas soluções da equação (4.11) mostram que $\lambda=\frac{j_{\nu n, k}}{l}$, onde $j_{\nu, k}$ são os zeros positivos da função de Bessel $J_{\nu}$, ordenados de maneira crescente, com $k=1,2, \ldots$ Já que o conjunto $\left\{J_{\nu}\left(j_{\nu, k} r\right)\right\}_{k=1,2, \ldots}$ define uma base ortogonal para o espaço $L^{2}(0,1)$, provamos que o conjunto

$$
\left\{\phi_{0}(\theta) \psi_{0, k}(r)=J_{0}\left(\frac{j_{0, k}}{l} r\right), \phi_{n, \pm}(\theta) \psi_{n, k}(r)=\mathrm{e}^{ \pm \mathrm{i} n \theta} J_{\nu n}\left(\frac{j_{\nu n, k}}{l} r\right)\right\}_{n \in \mathbb{N}_{0}}
$$

define um sistema completo de soluções linearmente independentes da equação dos autovalores (4.9) para $\Delta^{(0)}$ com condições de contorno de Dirichlet em $r=l$ sobre $L^{2}(0, l)$, e onde $\lambda=\frac{j_{\nu n, k}}{l}$ para ambos $\phi_{n, \pm} J_{\nu n}$ quando $n \neq 0$.

Por outro lado, as condições de contorno absoluta são dadas na equação (4.6). Aplicando nas solução da equação (4.11), obtemos

$$
\frac{\partial \omega}{\partial r}(l, \theta)=\lambda_{n} e^{\mathrm{i} n \theta} J_{|\nu n|}^{\prime}\left(\lambda_{n} l\right)=0
$$

o que nos leva a $\lambda_{n}=\frac{j_{|\nu n|, k}^{\prime}}{l}$, onde $j_{\nu n, k}^{\prime}$ são zeros de $J_{\nu n}^{\prime}(z)$.

O resultado para 2-formas é o dual das 0-formas. Note que, aplicando a transformada de Liouville inversa, obtemos um sistema completo para $\Delta^{(2)}$ com condições de contorno absoluta:

$$
\left\{\lambda_{n, k}^{(2)}=\frac{j_{\nu|n|, k}^{2}}{l^{2}}, \omega_{n, k}^{(2)}(r, \theta)=\phi_{n}(\theta) \rho_{\nu|n|, k}(r)=\mathrm{e}^{\mathrm{i} n \theta} x J_{\nu|n|}\left(\frac{j_{\nu|n|, k}}{l} r\right)\right\}_{n \in \mathbb{Z}, k \in \mathbb{N}_{0}} .
$$


Para uma 1-forma $\omega(r, \theta)=\omega_{r}(r, \theta) d r+\omega_{\theta}(r, \theta) d \theta, \omega_{\text {tan }}=\omega_{\theta}$ e $\omega_{\text {norm }}=\omega_{r}$. Note que nenhuma das soluções em (4.16) satisfaz as condições de contorno (4.8), para $\lambda_{n} \neq 0$. Assim, consideramos combinações lineares $\omega_{n, \pm}(r, \theta)=\omega_{1, n}(r, \theta) \pm \omega_{2, n}(r, \theta)$. Aplicando a condição de contorno relativa (4.8) em $\omega_{n, \pm}(r, \theta)$ obtemos, se $n \neq 0$, os autovalores

$$
\lambda_{n,+}^{2}=j_{|\nu n|, k}^{2} / l^{2}, \quad \lambda_{n,-}^{2}=\left(j_{|\nu n|, k}^{\prime}\right)^{2} / l^{2} .
$$

Se $n=0$, temos $\lambda_{0,+}^{2}=j_{1, k}^{2} / l^{2}$, e $\lambda_{0,-}^{2}=j_{0, k}^{2} / l^{2}$. Aplicando a condição de contorno absoluta de (4.8) para $\omega_{n, \pm}(r, \theta)$ obtemos, se $n \neq 0$, os autovalores

$$
\lambda_{n,+}^{2}=\left(j_{|\nu n|, k}^{\prime}\right)^{2} / l^{2}, \quad \lambda_{n,-}^{2}=j_{|\nu n|, k}^{2} / l^{2} .
$$

Se $n=0$, temos $\lambda_{0,+}^{2}=j_{0, k}^{2} / l^{2}$, e $\lambda_{0,-}^{2}=j_{1, k}^{2} / l^{2}$. As autoformas seguem da equação (4.16).

Lema 8. O espectro do Laplaciano $\Delta_{C_{\alpha} S_{l \operatorname{sen} \alpha}^{2}}^{(q)}$ sobre as q-formas com condições de contorno absoluta é:

$$
\begin{aligned}
\operatorname{Sp} \Delta_{C_{\alpha} S_{l \operatorname{sen} \alpha}^{2}}^{(0)}= & \left\{(2 n+1): \tilde{j}_{\mu_{n}, k,-}^{2} / l^{2}\right\}_{n, k=1}^{\infty} \cup\left\{j_{\frac{3}{2}, k}^{2} / l^{2}\right\}_{k=1}^{\infty}, \\
\operatorname{Sp} \Delta_{C_{\alpha} S_{l \operatorname{sen} \alpha}^{2}}^{(1)}= & \left\{j_{\frac{3}{2}, k}^{2} / l^{2}\right\}_{k=1}^{\infty} \cup\left\{(2 n+1): j_{\mu_{n}, k}^{2} / l^{2}\right\}_{n, k=1}^{\infty} \\
& \cup\left\{(2 n+1): \tilde{j}_{\mu_{n}, k,+}^{2} / l^{2}\right\}_{n, k=1}^{\infty} \cup\left\{(2 n+1): \tilde{j}_{\mu_{n}, k,-}^{2} / l^{2}\right\}_{n, k=1}^{\infty}, \\
\operatorname{Sp} \Delta_{C_{\alpha} S_{l \operatorname{sen} \alpha}^{2}}^{(2)}= & \left\{j_{\frac{1}{2}, k}^{2} / l^{2}\right\}_{k=1}^{\infty} \cup\left\{(2 n+1): j_{\mu_{n}, k}^{2} / l^{2}\right\}_{n, k=1}^{\infty} \\
& \cup\left\{(2 n+1): \tilde{j}_{\mu_{n}, k,+}^{2} / l^{2}\right\}_{n, k=1}^{\infty} \cup\left\{(2 n+1): j_{\mu_{n}, k}^{2} / l^{2}\right\}_{n, k=1}^{\infty}, \\
\operatorname{Sp} \Delta_{C_{\alpha} S_{l \operatorname{sen} \alpha}^{2}}^{(3)}= & \left\{(2 n+1): j_{\mu_{n}, k}^{2} / l^{2}\right\}_{n, k=1}^{\infty} \cup\left\{j_{\frac{1}{2}, k}^{2} / l^{2}\right\}_{k=1}^{\infty},
\end{aligned}
$$

O espectro do operador Laplaciano $\Delta_{C_{\alpha} S_{l \operatorname{sen} \alpha}^{2}}^{(q)} \quad$ sobre as $q$-formas com condições de contorno relativa é:

$$
\begin{aligned}
\operatorname{Sp} \Delta_{C_{\alpha} S_{l \operatorname{sen} \alpha}^{2}}^{(0)}= & \left\{(2 n+1): j_{\mu_{n}, k}^{2} / l^{2}\right\}_{n, k=1}^{\infty} \cup\left\{j_{\frac{1}{2}, k}^{2} / l^{2}\right\}_{k=1}^{\infty}, \\
\operatorname{Sp} \Delta_{C_{\alpha} S_{l \operatorname{sen} \alpha}^{2}}^{(1)}= & \left\{j_{\frac{1}{2}, k}^{2} / l^{2}\right\}_{k=1}^{\infty} \cup\left\{(2 n+1): j_{\mu_{n}, k}^{2} / l^{2}\right\}_{n, k=1}^{\infty} \\
& \cup\left\{(2 n+1): \tilde{j}_{\mu_{n}, k,+}^{2} / l^{2}\right\}_{n, k=1}^{\infty} \cup\left\{(2 n+1): j_{\mu_{n}, k}^{2} / l^{2}\right\}_{n, k=1}^{\infty}, \\
\operatorname{Sp} \Delta_{C_{\alpha} S_{l \operatorname{sen} \alpha}^{2}}^{(2)}= & \left\{j_{\frac{3}{2}, k}^{2} / l^{2}\right\}_{k=1}^{\infty} \cup\left\{(2 n+1): j_{\mu_{n}, k}^{2} / l^{2}\right\}_{n, k=1}^{\infty} \\
& \cup\left\{(2 n+1): \tilde{j}_{\mu_{n}, k,+}^{2} / l^{2}\right\}_{n, k=1}^{\infty} \cup\left\{(2 n+1): \tilde{j}_{\mu_{n}, k,-}^{2} / l^{2}\right\}_{n, k=1}^{\infty}, \\
\operatorname{Sp} \Delta_{C_{\alpha} S_{l \operatorname{sen} \alpha}^{2}}^{(3)}= & \left\{(2 n+1): \tilde{j}_{\mu_{n}, k,-}^{2} / l^{2}\right\}_{n, k=1}^{\infty} \cup\left\{j_{\frac{3}{2}, k}^{2} / l^{2}\right\}_{k=1}^{\infty},
\end{aligned}
$$

onde (lembramos que $\nu=\operatorname{cosec} \alpha)$

$$
\mu_{n}=\sqrt{\nu^{2} n(n+1)+\frac{1}{4}}
$$

e onde os $\tilde{j}_{\nu, k, \pm}$ são os zeros da função $G_{\nu}^{ \pm}(z)= \pm \frac{1}{2} J_{\nu}(z)+z J_{\nu}^{\prime}(z)$. 
Demonstração. Como antes, parametrizamos $C_{\alpha} S_{l \operatorname{sen} \alpha}^{2}$ por

$$
C_{\alpha} S_{l \operatorname{sen} \alpha}^{2}=\left\{\begin{array}{l}
x_{1}=r \operatorname{sen} \alpha \operatorname{sen} \theta_{2} \cos \theta_{1} \\
x_{2}=r \operatorname{sen} \alpha \operatorname{sen} \theta_{2} \operatorname{sen} \theta_{1} \\
x_{3}=r \operatorname{sen} \alpha \cos \theta_{2} \\
x_{4}=r \cos \alpha
\end{array}\right.
$$

onde $\left(r, \theta_{1}, \theta_{2}\right) \in[0, l] \times[0,2 \pi] \times[0, \pi], 0<\alpha \leq \pi / 2$ é um número real fixado e $0<a=$ $\operatorname{sen} \alpha \leq 1$. A métrica induzida é

$$
g=d r \otimes d r+\left(a^{2} r^{2} \operatorname{sen}^{2} \theta_{2}\right) d \theta_{1} \otimes d \theta_{1}+\left(a^{2} r^{2}\right) d \theta_{2} \otimes d \theta_{2} .
$$

O operador estrela de Hodge age nas formas da base como segue:

$$
\begin{aligned}
& *: 1 \mapsto a^{2} r^{2} \operatorname{sen} \theta_{2} d r \wedge d \theta_{1} \wedge d \theta_{2} ; \\
& *: d r \mapsto a^{2} r^{2} \operatorname{sen} \theta_{2} d \theta_{1} \wedge d \theta_{2}, *: d \theta_{1} \mapsto \frac{-1}{\operatorname{sen} \theta_{2}} d r \wedge d \theta_{2}, *: d \theta_{2} \mapsto \operatorname{sen} \theta_{2} d r \wedge d \theta_{1} ; \\
& *: d r \wedge d \theta_{1} \mapsto \frac{1}{\operatorname{sen} \theta_{2}} d \theta_{2}, *: d r \wedge d \theta_{2} \mapsto-\operatorname{sen} \theta_{2} d \theta_{1}, *: d \theta_{1} \wedge d \theta_{2} \mapsto \frac{1}{a^{2} r^{2} \operatorname{sen} \theta_{2}} d r \\
& *: d r \wedge d \theta_{1} \wedge d \theta_{2} \mapsto \frac{1}{a^{2} r^{2} \operatorname{sen} \theta_{2}} .
\end{aligned}
$$

O Laplaciano nas 0-formas é dado pela equação

$$
\Delta^{(0)}(\omega)=-\left(\frac{2}{r} \partial_{r} \omega+\partial_{r}^{2} \omega+\frac{\cos \theta_{2}}{a^{2} r^{2} \operatorname{sen} \theta_{2}} \partial_{\theta_{2}} \omega+\frac{\partial_{\theta_{2}}^{2} \omega}{a^{2} r^{2}}+\frac{\partial_{\theta_{1}}^{2} \omega}{a^{2} r^{2} \operatorname{sen}^{2} \theta_{2}}\right),
$$

e no caso das 1-formas é

$$
\begin{gathered}
\Delta^{(1)}\left(\omega_{r} d r+\omega_{\theta_{1}} d \theta_{1}+\omega_{\theta_{2}} d \theta_{2}\right)= \\
\left(-\partial_{r}^{2} \omega_{r}+\frac{2}{r^{2}} \omega_{r}-\frac{2}{r} \partial_{r} \omega_{r}-\frac{1}{a^{2} r^{2}} \partial_{\theta_{2}}^{2} \omega_{r}-\frac{\cos \theta_{2}}{a^{2} r^{2} \operatorname{sen} \theta_{2}} \partial_{\theta_{2}} \omega_{r}\right. \\
\left.-\frac{1}{a^{2} r^{2} \operatorname{sen}^{2} \theta_{2}} \partial_{\theta_{1}}^{2} \omega_{r}+\frac{2}{a^{2} r^{3} \operatorname{sen}^{2} \theta_{2}} \partial_{\theta_{1}} \omega_{\theta_{1}}+\frac{2}{a^{2} r^{3}} \partial_{\theta_{2}} \omega_{\theta_{2}}+\frac{2 \cos \theta_{2}}{a^{2} r^{3} \operatorname{sen} \theta_{2}} \omega_{\theta_{2}}\right) d r \\
+\left(-\partial_{r}^{2} \omega_{\theta_{1}}-\frac{2}{r} \partial_{\theta_{1}} \omega_{r}-\frac{1}{a^{2} r^{2}} \partial_{\theta_{2}}^{2} \omega_{\theta_{1}}\right. \\
\left.+\frac{\cos \theta_{2}}{a^{2} r^{2} \operatorname{sen} \theta_{2}}\left(\partial_{\theta_{2}} \omega_{\theta_{1}}-2 \partial_{\theta_{1}} \omega_{\theta_{2}}\right)-\frac{1}{a^{2} r^{2} \operatorname{sen}^{2} \theta_{2}} \partial_{\theta_{1}}^{2} \omega_{\theta_{1}}\right) d \theta_{1} \\
+\left(-\partial_{r}^{2} \omega_{\theta_{2}}-\frac{2}{r} \partial_{\theta_{2}} \omega_{r}-\frac{1}{a^{2} r^{2}} \partial_{\theta_{2}}^{2} \omega_{\theta_{2}}-\frac{\cos \theta_{2}}{a^{2} r^{2} \operatorname{sen} \theta_{2}} \partial_{\theta_{2}} \omega_{\theta_{2}}\right. \\
\left.+\frac{1}{a^{2} r^{2} \operatorname{sen}^{2} \theta_{2}}\left(\omega_{\theta_{2}}-\partial_{\theta_{1}}^{2} \omega_{\theta_{2}}\right)+\frac{2 \cos \theta_{2}}{a^{2} r^{2} \operatorname{sen}^{3} \theta_{2}} \partial_{\theta_{1}} \omega_{\theta_{1}}\right) d \theta_{2} .
\end{gathered}
$$


Aplicando a decomposição descrita na equação (2.5), obtemos das equações (2.6), e (2.7), os conjuntos de condições de contorno.

Para as 0-formas:

$$
\text { abs. : } \partial_{r} \omega\left(l, \theta_{1}, \theta_{2}\right)=0, \quad \text { rel. : } \omega\left(l, \theta_{1}, \theta_{2}\right)=0 \text {. }
$$

Para as 1-formas:

$$
\text { abs. : }\left\{\begin{array} { l } 
{ \omega _ { r } ( l , \theta _ { 1 } , \theta _ { 2 } ) = 0 } \\
{ \partial _ { r } \omega _ { \theta _ { 1 } } ( l , \theta _ { 1 } , \theta _ { 2 } ) = 0 } \\
{ \partial _ { r } \omega _ { \theta _ { 2 } } ( l , \theta _ { 1 } , \theta _ { 2 } ) = 0 , }
\end{array} \quad \text { rel. : } \left\{\begin{array}{l}
\omega_{\theta_{1}}\left(l, \theta_{1}, \theta_{2}\right)=0 \\
\omega_{\theta_{2}}\left(l, \theta_{1}, \theta_{2}\right)=0 \\
\partial_{r}\left(r^{2} \omega_{r}\right)\left(l, \theta_{1}, \theta_{2}\right)=0,
\end{array}\right.\right.
$$

onde $\omega\left(r, \theta_{1}, \theta_{2}\right)=\omega_{r}\left(r, \theta_{1}, \theta_{2}\right) d r+\omega_{\theta_{1}}\left(r, \theta_{1}, \theta_{2}\right) d \theta_{1}+\omega_{\theta_{2}}\left(r, \theta_{1}, \theta_{2}\right) d \theta_{2}$.

O próximo passo é resolver as equações dos autovalores. Para as 0 -formas:

$$
\Delta^{(0)}(\omega)=-\partial_{r}^{2} \omega-\frac{2}{r} \partial_{r} \omega+\frac{1}{a^{2} r^{2}} \Delta_{S^{2}}^{(0)}(\omega)=\lambda^{2} \omega
$$

Decompomos o problema nos autoespaços de $\Delta_{S^{2}}^{(0)}$. Seja $Y_{n}^{k}\left(\theta_{1}, \theta_{2}\right)=\mathrm{e}^{i k \theta_{1}} P_{n}^{|k|}\left(\cos \theta_{2}\right)$, onde $P_{n}^{|k|}\left(\cos \theta_{2}\right)$ são os polinômios associados de Legendre e $|k| \leq n$. $Y_{n}^{k}\left(\theta_{1}, \theta_{2}\right)$ é um sistema completo de autoformas para $\Delta_{S^{2}}^{(0)}$ e os autovalores são $n(n+1)$, com multiplicidade $2 n+1$ e $n \in \mathbb{Z}, n \geq 0$. Então,

$$
\Delta^{(0)}=\sum_{n \geq 0} T_{n} \Pi_{n}
$$

com

$$
T_{n}=-\partial_{r}^{2} \omega-\frac{2}{r} \partial_{r} \omega+\frac{\nu^{2} n(n+1)}{r^{2}},
$$

e a equação dos autovalores fica

$$
T_{n}(u)=\left(-\partial_{r}^{2} \omega-\frac{2}{r} \partial_{r} \omega+\frac{\nu^{2} n(n+1)}{r^{2}}\right) u=\lambda^{2} u .
$$

Isto pode ser resolvido em termos de funções de Bessel e a solução é $u_{n}(r)=r^{-\frac{1}{2}} J_{\mu_{n}}\left(\lambda_{n} r\right)$. Assim a solução para a equação do 0-Laplaciano é

$$
\psi_{1, n}^{(0)}\left(r, \theta_{1}, \theta_{2}\right)=r^{-\frac{1}{2}} J_{\mu_{n}}\left(\lambda_{n} r\right) Y_{n}^{k}\left(\theta_{1}, \theta_{2}\right), \text { e } \psi_{E}^{(0)}\left(r, \theta_{1}, \theta_{2}\right)=r^{-\frac{1}{2}} J_{\frac{1}{2}}\left(\lambda_{n} r\right)
$$

Para as 1-formas temos

$$
\Delta^{(1)}(\omega)=\lambda^{2} \omega
$$

$\operatorname{com} \omega=\omega_{r} d r+\omega_{\theta_{1}} d \theta_{1}+\omega_{\theta_{2}} d \theta_{2}$. Escreva $\omega=f_{\theta_{1} \theta_{2}}(r) \phi\left(\theta_{1}, \theta_{2}\right)+f_{r}(r) h\left(\theta_{1}, \theta_{2}\right) d r$ onde $\phi=f_{\theta_{1}} d \theta_{1}+f_{\theta_{2}} d \theta_{2}$ e $h\left(\theta_{1}, \theta_{2}\right)$ é uma 0 -forma em $S^{2}$. Assim, substituindo em (4.21), temos o sistema

$$
\left\{\begin{array}{l}
\left(-\partial_{r}^{2} f_{\theta_{1} \theta_{2}}\right) \phi+\frac{\Delta_{S^{2}}^{(1)}(\phi)}{a^{2} r^{2}} f_{\theta_{1} \theta_{2}}-\frac{2 f_{r} d(h)}{r}=\lambda^{2} f_{\theta_{1} \theta_{2}} \phi \\
d r\left(\left(-\partial_{r}^{2} f_{r}-\frac{2}{r} \partial_{r} f_{r}+\frac{2}{r^{2}} f_{r}\right) h+\frac{\Delta_{S^{2}}^{(0)}(h)}{a^{2} r^{2}} f_{r}-\frac{2 f_{\theta_{1} \theta_{2}} d_{S^{2}}^{\dagger}(\phi)}{a^{2} r^{3}}\right)=d r\left(\lambda^{2} f_{r} h\right) .
\end{array}\right.
$$


Considere $f_{r}=0$ ou $h=0$ e $\phi$ uma autoforma coexata em $S^{2}$ com autovalor não nulo. Então temos a equação, para $n \geq 1$,

$$
\left(-d_{r}^{2} f_{\theta_{1} \theta_{2}}\right) \phi+\frac{\nu^{2} n(n+1) f_{\theta_{1} \theta_{2}}}{r^{2}} \phi=\lambda^{2} f_{\theta_{1} \theta_{2}} \phi .
$$

Resolvendo esta equação em $r$ encontramos que $f_{\theta_{1} \theta_{2}}=r^{\frac{1}{2}} J_{\mu_{n}}\left(\lambda_{n} r\right)$ e, então,

$$
\psi_{1, n}^{(1)}=r^{\frac{1}{2}} J_{\mu_{n}}\left(\lambda_{n} r\right) \phi
$$

Note que $\phi=d_{S^{2}}^{\dagger}\left(\operatorname{sen} \theta_{2} Y_{n}^{k}\left(\theta_{1}, \theta_{2}\right) d \theta_{1} \wedge d \theta_{2}\right)$. Agora consideramos $f_{r} \neq 0, f_{\theta_{1} \theta_{2}} \neq 0$, e $h$ uma 0-autoforma coexata de $S^{2}$ com autovalor não nulo tal que $d(h)=\phi$. Assim, $\Delta_{S^{2}}^{(1)}(\phi)=$ $n(n+1) \phi, d_{S^{2}}^{\dagger}(d(h))=n(n+1) h$, e o sistema (4.22) fica

$$
\left\{\begin{array}{l}
\left(-\partial_{r}^{2} f_{\theta_{1} \theta_{2}}\right) \phi+\frac{n(n+1) f_{\theta_{1} \theta_{2}}}{a^{2} r^{2}} \phi-\frac{2 f_{\theta_{1} \theta_{2}} \phi}{r}=\lambda^{2} f_{\theta_{1} \theta_{2}} \phi \\
\left(-\partial_{r}^{2} f_{r}-\frac{2}{r} \partial_{r} f_{r}+\frac{2}{r^{2}} f_{r}\right) h+\frac{n(n+1) h}{a^{2} r^{2}} f_{r}-\frac{2 n(n+1) f_{\theta_{1} \theta_{2}}(\phi)}{a^{2} r^{3}}=\lambda^{2} f_{r} h .
\end{array}\right.
$$

Mudando a base $\left(f_{\theta_{1} \theta_{2}}, f_{r}\right)=\left(r^{-\frac{1}{2}} g_{r}, \partial_{r}\left(r^{-\frac{1}{2}} g_{r}\right)\right)$ resolvemos o sistema e a solução é $g_{r}=$ $J_{\mu_{n}}\left(\lambda_{n} r\right)$. Assim a solução para o sistema (4.22), neste caso, é

$$
\psi_{2, n}^{(1)}=r^{-\frac{1}{2}} J_{\mu_{n}}\left(\lambda_{n} r\right) \phi\left(\theta_{1}, \theta_{2}\right)+\partial_{r}\left(r^{-\frac{1}{2}} J_{\mu_{n}}\left(\lambda_{n} r\right)\right) h\left(\theta_{1}, \theta_{2}\right) d r
$$

onde $h\left(\theta_{1}, \theta_{2}\right)=Y_{n}^{k}\left(\theta_{1}, \theta_{2}\right)$.

Considere agora $f_{r} \neq 0, f_{\theta_{1} \theta_{2}} \neq 0$ e $\gamma$ uma 0-autoforma coexata de $S^{2}$ com autovalor não nulo tal que $d(\gamma)=\phi$ e $d_{S^{2}}^{\dagger} d(\gamma)=h$. Então $h=n(n+1) \psi$ e o sistema (4.22) fica

$$
\left\{\begin{array}{l}
\left(-\partial_{r}^{2} f_{\theta_{1} \theta_{2}}\right) \phi+\frac{n(n+1) f_{\theta_{1} \theta_{2}}}{a^{2} r^{2}} \phi-\frac{2 f_{r} n(n+1)}{r} \phi=\lambda^{2} f_{\theta_{1} \theta_{2}} \phi, \\
d r\left(\left(-\partial_{r}^{2} f_{r}-\frac{2}{r} \partial_{r} f_{r}+\frac{2}{r^{2}} f_{r}\right) h+\frac{n(n+1) f_{r}}{a^{2} r^{2}} h-\frac{2 f_{\theta_{1} \theta_{2}}}{a^{2} r^{3}} h\right)=d r\left(\lambda^{2} f_{r} h\right) .
\end{array}\right.
$$

Mudando a base $\left(f_{\theta_{1} \theta_{2}}, f_{r}\right)=\left(\partial_{r}\left(r^{\frac{1}{2}} g_{r}\right), r^{-\frac{3}{2}} g_{r}\right)$ resolvemos o sistema (4.23) e a solução é $g_{r}=J_{\mu_{n}}\left(\lambda_{n} r\right)$. Assim a solução para o sistema (4.22) fica

$$
\psi_{3, n}^{(1)}=\partial_{r}\left(r^{\frac{1}{2}} J_{\mu_{n}}\left(\lambda_{n} r\right)\right)\left(\lambda_{n} r\right) \phi\left(\theta_{1}, \theta_{2}\right)+r^{-\frac{3}{2}} J_{\mu_{n}}\left(\lambda_{n} r\right) h\left(\theta_{1}, \theta_{2}\right) d r
$$

onde $\psi\left(\theta_{1}, \theta_{2}\right)=Y_{n}^{k}\left(\theta_{1}, \theta_{2}\right)$.

No caso em que $\Delta_{S^{2}}^{(1)}(\phi)=\Delta_{S^{2}}^{(0)}(h)=0$ temos a equação

$$
d r\left(\left(-\partial_{r}^{2} f_{r}-\frac{2}{r} \partial_{r} f_{r}+\frac{2}{r^{2}} f_{r}\right) h\right)=d r\left(\lambda^{2} f_{r} h\right) .
$$

Assim, mudando a base $f_{r}=\partial_{r}\left(r^{-\frac{1}{2}} g_{r}\right)$ encontramos $g_{r}=J_{\frac{1}{2}}\left(\lambda_{0} r\right)$ e a solução é

$$
\psi_{O}^{(1)}=\partial_{r}\left(r^{-\frac{1}{2}} J_{\frac{1}{2}}\left(\lambda_{0} r\right)\right) h .
$$


Sabemos que a decomposição de Hodge das formas $L^{2}$ em formas exatas e coexatas valem como no caso suave (ver seção 1.4 e [10]), concluímos que a equação (4.21) possui o seguinte sistema completo de soluções $L^{2}$ linearmente independente, $\operatorname{com} \lambda \neq 0$,

$$
\begin{aligned}
& \psi_{1, n}^{(1)}=r^{\frac{1}{2}} J_{\mu_{n}}(\lambda r) d_{S^{2}}^{\dagger}\left(\operatorname{sen} \theta_{2} Y_{n}^{k}\left(\theta_{1}, \theta_{2}\right) d \theta_{1} \wedge d \theta_{2}\right) \\
& \psi_{2, n}^{(1)}=\partial_{r}\left(r^{-\frac{1}{2}} J_{\mu_{n}}(\lambda r)\right) Y_{n}^{k}\left(\theta_{1}, \theta_{2}\right) d r+r^{-\frac{1}{2}} J_{\mu_{n}}(\lambda r) d\left(Y_{n}^{k}\left(\theta_{1}, \theta_{2}\right)\right) \\
& \psi_{3, n}^{(1)}=r^{-\frac{3}{2}} J_{\mu_{n}}(\lambda r)(n(n+1)) \nu^{2} Y_{n}^{k}\left(\theta_{1}, \theta_{2}\right) d r+\partial_{r}\left(r^{\frac{1}{2}} J_{\mu_{n}}(\lambda r)\right) d\left(Y_{n}^{k}\left(\theta_{1}, \theta_{2}\right)\right) \\
& \psi_{O}^{(1)}=\partial_{r}\left(r^{-\frac{1}{2}} J_{\frac{1}{2}}(\lambda r)\right) d r
\end{aligned}
$$

A seguir determinaremos os autovalores.

\section{0-formas}

Temos apenas dois tipos de formas em (4.20), que são:

$$
\psi_{1, n}^{(0)}=r^{-\frac{1}{2}} J_{\mu_{n}}(\lambda r) \phi_{n}^{(0)}, \quad \psi_{E}^{(0)}=r^{-\frac{1}{2}} J_{\frac{1}{2}}(\lambda r) \phi_{n}^{(0)} .
$$

Usando as condições de contorno absoluta em (4.17) obtemos

$$
\begin{aligned}
& \partial_{r}\left(\psi_{1, n}^{(0)}\right)\left(l, \theta_{1}, \theta_{2}\right)=\partial_{r}\left(r^{-\frac{1}{2}} J_{\mu_{n}}(\lambda r) \phi_{n}^{(0)}\right)\left(l, \theta_{1}, \theta_{2}\right)=0 \\
& \partial_{r}\left(\psi_{E}^{(0)}\right)\left(l, \theta_{1}, \theta_{2}\right)=\partial_{r}\left(r^{-\frac{1}{2}} J_{\frac{1}{2}}(\lambda r) \phi_{n}^{(0)}\right)\left(l, \theta_{1}, \theta_{2}\right)=0
\end{aligned}
$$

e assim precisamos do quadrado das soluções de $-\frac{1}{2} l^{-\frac{3}{2}} J_{\mu_{n}}(l \lambda)+l^{-\frac{1}{2}} \lambda J_{\mu_{n}}^{\prime}(l \lambda)=0$ e de $-\lambda^{-\frac{1}{2}} J_{\frac{3}{2}}(l \lambda)=0$ que são

$$
\lambda^{2}=\frac{\tilde{j}_{\mu_{n}, k,-}^{2}}{l^{2}} \quad \text { e } \quad \lambda^{2}=\frac{j_{\frac{3}{2}, k}^{2}}{l^{2}} .
$$

Considerando as condições de contorno relativa em (4.17) e aplicando em $\psi_{1, n}^{(0)}$ e $\psi_{E}^{(0)}$, obtemos que $\psi_{1, n}^{(0)}\left(l, \theta_{1}, \theta_{2}\right)=\psi_{E}^{(0)}\left(l, \theta_{1}, \theta_{2}\right)=0$ e então os autovalores são

$$
\lambda^{2}=\frac{j_{\mu_{n}, k}^{2}}{l^{2}} \quad \text { e } \quad \lambda^{2}=\frac{j_{\frac{1}{2}, k}^{2}}{l^{2}} .
$$

\section{1-formas}

Neste caso temos quatro tipos de formas em (4.24) $(s=1,2)$ :

$$
\begin{array}{ll}
\psi_{1, n}^{(1)}=r^{\frac{1}{2}} J_{\mu_{n}}(\lambda r) \phi_{n}^{(1)}, & \psi_{2, n}^{(1)}=\partial_{r}\left(r^{-\frac{1}{2}} J_{\mu_{n}}(\lambda r)\right) \phi_{n}^{(0)} d r+r^{-\frac{1}{2}} J_{\mu_{n}}(\lambda r) d \phi_{n}^{(0)}, \\
\psi_{O}^{(1)}=\partial_{r}\left(r^{-\frac{1}{2}} J_{\frac{1}{2}}(\lambda r)\right) \phi_{n}^{(0)} d r, & \psi_{3, n}^{(1)}=n(n+1) r^{-\frac{3}{2}} J_{\mu_{n}}(\lambda r) \nu^{2} \phi_{n}^{(0)} d r+\partial_{r}\left(r^{\frac{1}{2}} J_{\mu_{n}}(\lambda r)\right) d \phi_{n}^{(0)} .
\end{array}
$$


Usando as condições de contorno absoluta em (4.18) temos, para os quatro tipos,

$$
\begin{aligned}
\partial_{r}\left(\left(\psi_{1, n}^{(1)}\right)_{\theta_{s}}\right)\left(l, \theta_{1}, \theta_{2}\right) & =\partial_{r}\left(r^{\frac{1}{2}} J_{\mu_{n}}(\lambda r)\right)(l)=0 \\
\left(\psi_{2, n}^{(1)}\right)_{r}\left(l, \theta_{1}, \theta_{2}\right)=\partial_{r}\left(\left(\beta_{n}^{(1)}\right)_{\theta_{s}}\right)\left(l, \theta_{1}, \theta_{2}\right) & =\partial_{r}\left(r^{-\frac{1}{2}} J_{\mu_{n}}(\lambda r)\right)(l)=0 \\
\left(\psi_{3, n}^{(1)}\right)_{r}\left(l, \theta_{1}, \theta_{2}\right) & =J_{\mu_{n}}(l \lambda)=0 \\
\partial_{r}\left(\gamma_{n}^{(1)}\right)_{\theta_{s}}\left(l, \theta_{1}, \theta_{2}\right) & =-\frac{1}{4} J_{\mu_{n}}(l \lambda)+\lambda J_{\mu_{n}}^{\prime}(l \lambda)+\lambda^{2} J_{\mu_{n}}^{\prime \prime}(l \lambda)=0 \\
\partial_{r}\left(\psi_{O}^{(1)}\right)\left(l, \theta_{1}, \theta_{2}\right) & =\partial_{r}\left(r^{-\frac{1}{2}} J_{\frac{1}{2}}(\lambda r)\right)(1)=0 .
\end{aligned}
$$

Desta forma obtemos os quadrados dos zeros das seguinte equações,

$$
\begin{aligned}
\frac{1}{2} J_{\mu_{n}}(l \lambda)+l \lambda J_{\mu_{n}}^{\prime}(l \lambda) & =0 \\
-\frac{1}{2} J_{\mu_{n}}(l \lambda)+l \lambda J_{\mu_{n}}^{\prime}(l \lambda) & =0 \\
J_{\mu_{n}}(l \lambda) & =0, \\
\lambda^{-\frac{1}{2}} J_{-\frac{1}{2}}(l \lambda) & =0 \\
-\lambda^{-\frac{1}{2}} J_{\frac{3}{2}}(l \lambda) & =0
\end{aligned}
$$

que são

$$
\lambda^{2}=\frac{\tilde{j}_{\mu_{n}, k,+}^{2}}{l^{2}}, \quad \lambda^{2}=\frac{\tilde{j}_{\mu_{n}, k,-}^{2}}{l^{2}}, \quad \lambda^{2}=\frac{j_{\mu_{n}, k}^{2}}{l^{2}} \quad \text { e } \quad \lambda^{2}=\frac{j_{\frac{3}{2}, k}^{2}}{l^{2}}
$$

Usando as condições de contorno relativa em (4.18) obtemos, para os quatro tipos,

$$
\begin{aligned}
\left(\psi_{1, n}^{(1)}\right)_{\theta_{s}}\left(l, \theta_{1}, \theta_{2}\right) & =\left(r^{\frac{1}{2}} J_{\mu_{n}}(\lambda r)\right)(l)=0 \\
\left(\psi_{2, n}^{(1)}\right)_{\theta_{s}}\left(l, \theta_{1}, \theta_{2}\right) & =J_{\mu_{n}}(l \lambda)=0 \\
\partial_{r}\left(r^{2}\left(\psi_{2, n}^{(1)}\right)_{r}\right)\left(l, \theta_{1}, \theta_{2}\right) & =-\frac{1}{4} J_{\mu_{n}}(l \lambda)+l \lambda J_{\mu_{n}}^{\prime}(l \lambda)+(l \lambda)^{2} J_{\mu_{n}}^{\prime \prime}(l \lambda)=0 \\
\partial_{r}\left(\left(\psi_{3, n}^{(1)}\right)_{\theta_{s}}\right)\left(l, \theta_{1}, \theta_{2}\right) & =\frac{1}{2} J_{\mu_{n}}(l \lambda)+l \lambda J_{\mu_{n}}^{\prime}(l \lambda)=0 \\
\partial_{r}\left(r^{2}\left(\psi_{3, n}^{(1)}\right)_{r}\right)\left(l, \theta_{1}, \theta_{2}\right) & =\frac{1}{2} J_{\mu_{n}}(l \lambda)+l \lambda J_{\mu_{n}}^{\prime}(l \lambda)=0 \\
\partial_{r}\left(r^{2}\left(\psi_{O}^{(1)}\right)_{r}\right)\left(l, \theta_{1}, \theta_{2}\right) & =\partial_{r}\left(r^{2} \partial_{r}\left(r^{-\frac{1}{2}} J_{\frac{1}{2}}(\lambda r)\right)\right)(1)=0
\end{aligned}
$$

e assim é necessário determinar o quadrado dos zeros de $J_{\mu_{n}}(l \lambda)=0,-\frac{1}{4} J_{\mu_{n}}(l \lambda)+l \lambda J_{\mu_{n}}^{\prime}(l \lambda)+$ $(l \lambda)^{2} J_{\mu_{n}}^{\prime \prime}(l \lambda)=0, \frac{1}{2} J_{\mu_{n}}(l \lambda)+l \lambda J_{\mu_{n}}^{\prime}(l \lambda)=0$ e $J_{\frac{1}{2}}(l \lambda)=0$, que são

$$
\lambda^{2}=\frac{j_{\mu_{n}, k}^{2}}{l^{2}} \text { (duas vezes), } \quad \lambda^{2}=\frac{\tilde{j}_{\mu_{n}, k,+}^{2}}{l^{2}} \quad \text { e } \quad \lambda^{2}=\frac{j_{\frac{1}{2}, k}^{2}}{l^{2}} .
$$

Isto conclui a prova para as 0 -formas e 1-formas. O resultado para as 2 -formas e as 3 -formas segue por dualidade. 


\subsubsection{Torção Analítica do cone sobre o círculo}

Teorema 15. A Torção Analítica do cone $C_{\alpha} S_{l \operatorname{sen} \alpha}^{1}$ de ângulo $\alpha$ e comprimento $l>0$, sobre o círculo, com a métrica padrão $g_{E}$ induzida pela imersão, com condiçôes de contorno absoluta (relativa) e uma representação ortogonal de posto 1 é:

$$
\log T_{\mathrm{abs}}\left(C_{\alpha} S_{l \operatorname{sen} \alpha}^{1}\right)=-\log T_{\mathrm{rel}}\left(C_{\alpha} S_{l \operatorname{sen} \alpha}^{1}\right)=\frac{1}{2} \log \left(\pi l^{2} \operatorname{sen} \alpha\right)+\frac{1}{2} \operatorname{sen} \alpha .
$$

Em particular, para o disco $D_{l}^{2}$ temos:

$$
\log T_{\text {abs }}\left(D_{l}^{2}\right)=-\log T_{\text {rel }}\left(D_{l}^{2}\right)=\frac{1}{2} \log \pi l^{2}+\frac{1}{2} .
$$

Demonstração. Claramente, a Torção Analítica com condições de contorno absoluta no bordo coincide, a menos de sinal, com a Torção Analítica com condições de contorno relativa no bordo. Usando a análise na seção 4.3.1, as função zetas relevantes são

$$
\begin{aligned}
& \zeta\left(s, \Delta^{(1)}\right)=\sum_{k=1}^{\infty} \frac{j_{0, k}^{-2 s}}{l^{-2 s}}+\sum_{k=1}^{\infty} \frac{j_{1, k}^{-2 s}}{l^{-2 s}}+2 \sum_{n, k=1}^{\infty} \frac{j_{\nu n, k}^{-2 s}}{l^{-2 s}}+2 \sum_{n, k=1}^{\infty} \frac{\left(j_{\nu n, k}^{\prime}\right)^{-2 s}}{l^{-2 s}}, \\
& \zeta\left(s, \Delta^{(2)}\right)=\sum_{k=1}^{\infty} \frac{j_{0, k}^{-2 s}}{l^{-2 s}}+2 \sum_{n, k=1}^{\infty} \frac{j_{\nu n, k}^{-2 s}}{l^{-2 s}}
\end{aligned}
$$

e pela equação (2.11), a torção é $\left(a=\operatorname{sen} \alpha=\frac{1}{\nu}\right)$

$$
\log T_{\mathrm{abs}}\left(\left(C_{\alpha} S_{l a}^{1}, g_{E}\right) ; \rho\right)=-\frac{1}{2} \zeta^{\prime}\left(0, \Delta^{(1)}\right)+\zeta^{\prime}\left(0, \Delta^{(2)}\right)
$$

Defina a função

$$
\begin{aligned}
t(s) & =-\frac{1}{2} \zeta\left(s, \Delta^{(1)}\right)+\zeta\left(s, \Delta^{(2)}\right) \\
& =\frac{1}{2} l^{2 s} \sum_{k=1}^{\infty} j_{0, k}^{-2 s}-\frac{1}{2} l^{2 s} \sum_{k=1}^{\infty} j_{1, k}^{-2 s}+l^{2 s} \sum_{n, k=1}^{\infty} j_{\nu n, k}^{-2 s}-l^{2 s} \sum_{n, k=1}^{\infty}\left(j_{\nu n, k}^{\prime}\right)^{-2 s} \\
& =l^{2 s}\left(\frac{1}{2} z_{0}(s)-\frac{1}{2} z_{1}(s)+Z(s)-\hat{Z}(s)\right)
\end{aligned}
$$

então

$$
\begin{aligned}
\log T_{\mathrm{abs}}\left(\left(C_{\alpha} S_{l a}^{1}, g_{E}\right) ; \rho\right)=t^{\prime}(0)=\log l^{2} & \left(\frac{1}{2} z_{0}(0)-\frac{1}{2} z_{1}(0)+Z(0)-\hat{Z}(0)\right) \\
+ & \frac{1}{2} z_{0}^{\prime}(0)-\frac{1}{2} z_{1}^{\prime}(0)+Z^{\prime}(0)-\hat{Z}^{\prime}(0) .
\end{aligned}
$$

Usando a equação (1.22) da seção 1.5, calculamos $z_{0 / 1}(0)$ e $z_{0 / 1}^{\prime}(0)$. Obtemos

$$
\log T_{\mathrm{abs}}\left(\left(C_{\alpha} S_{l a}^{1}, g_{E}\right) ; \rho\right)=\left(\frac{1}{4}+Z(0)-\hat{Z}(0)\right) \log l^{2}+Z^{\prime}(0)-\hat{Z}^{\prime}(0)-\frac{1}{2} \log 2 .
$$

Falta determinar as diferenças $Z(0)-\hat{Z}(0)$ e $Z^{\prime}(0)-\hat{Z}^{\prime}(0)$. Usaremos o teorema 5. As seqüências relevantes são as seqüências duplas $S=\left\{j_{\nu n, k}^{2}\right\}$ e $\hat{S}=\left\{\left(j_{\nu n, k}^{\prime}\right)^{2}\right\}$ e a seqüência 
simples é $U=\{\nu n\}_{n=1}^{\infty}$. Ainda $Z(s)=\zeta(s, S), \hat{Z}(s)=\zeta(s, \hat{S})$. Usando estimativas clássicas para os zeros da função de Bessel [48], temos que o genus de $S$ é 1 , o genus de $U$ é 1 , e os genus relativos de $S$ são $(1,0,0)$. Mostremos que $U, S_{n}$, e $\hat{S}_{n}$ são seqüências totalmente regular do tipo espectral. Para $U$, é direto (veja ainda [43] seção 3.1), como $\zeta(s, U)=\nu^{-s} \zeta_{R}(s)$. Para $S$ e $\hat{S}$ note que temos as seguintes representações como produtos (a primeira é clássica, ver [48], a segunda segue usando o teorema da fatorização de Hadamard, ver [12])

$$
I_{\nu}(z)=\frac{z^{\nu}}{2^{\nu} \Gamma(\nu+1)} \prod_{k=1}^{\infty}\left(1+\frac{z^{2}}{j_{\nu, k}^{2}}\right), \quad I_{\nu}^{\prime}(z)=\frac{z^{\nu-1}}{2^{\nu} \Gamma(\nu)} \prod_{k=1}^{\infty}\left(1+\frac{z^{2}}{\left(j_{\nu, k}^{\prime}\right)^{2}}\right) .
$$

Usando estas representações, obtemos as seguintes representações para as funções Gamma associadas as seqüências $S_{n}$ e $\hat{S}_{n}$. Para utilizarmos depois, apresentamos as representações para as funções Gamma associada as seqüências $S_{n} / u_{n}^{2}$, e $S_{n}^{\prime} / u_{n}^{2}$. Pela definição da equação (1.17), $\operatorname{com} z=\sqrt{-\lambda}$, temos

$$
\begin{aligned}
\log \Gamma\left(-\lambda, S_{n} /(\nu n)^{2}\right)= & -\log \prod_{k=1}^{\infty}\left(1+\frac{(-\lambda)(\nu n)^{2}}{j_{\nu n, k}^{2}}\right) \\
= & -\log I_{\nu n}(\nu n \sqrt{-\lambda})+(\nu n) \log \sqrt{-\lambda} \\
& +\nu n \log (\nu n)-\nu n \log 2-\log \Gamma(\nu n+1), \\
\log \Gamma\left(-\lambda, \hat{S}_{n} /(\nu n)^{2}\right)= & -\log \prod_{k=1}^{\infty}\left(1+\frac{(-\lambda)(\nu n)^{2}}{\left(j_{\nu n, k}^{\prime}\right)^{2}}\right) \\
= & -\log I_{\nu n}^{\prime}(\nu n \sqrt{-\lambda})+(\nu n-1) \log \sqrt{-\lambda} \\
& +\nu n \log (\nu n)-\nu n \log 2-\log \Gamma(\nu n+1) .
\end{aligned}
$$

Uma primeira conseqüência desta representação é que temos uma expansão assintótica para as funções Gamma $\log \Gamma\left(-\lambda, S_{n}\right)$ e $\log \Gamma\left(-\lambda, \hat{S}_{n}\right)$ e, portanto, $S_{n}$ e $\hat{S}_{n}$ são seqüências do tipo espectral. Mais ainda, considerando as expansões, vemos que ambas são seqüências totalmente regulares de ordem infinita.

A seguir, provamos que $S$ e $\hat{S}$ são decompostas espectralmente sobre $U$ com potência $\kappa=2$ e comprimento $\ell=2$, como na definição 13. Temos que mostrar que as funções $\log \Gamma\left(-\lambda, S_{n} / u_{n}^{2}\right)$ e $\log \Gamma\left(-\lambda, \hat{S}_{n} / u_{n}^{2}\right)$ tem a expansão uniforme apropriada para $n$ grande. Isto segue usando as expansões uniformes para as funções de Bessel, dadas em [35] no capítulo 10 na seção 7 ,

$$
I_{\nu}(\nu z)=\frac{\mathrm{e}^{\nu \sqrt{1+z^{2}}} \mathrm{e}^{\nu \log \frac{z}{1+\sqrt{1+z^{2}}}}}{\sqrt{2 \pi \nu}\left(1+z^{2}\right)^{\frac{1}{4}}}\left(1+U_{1}(z) \frac{1}{\nu}+O\left(\nu^{-2}\right)\right),
$$

$\operatorname{com} U_{1}(z)=\frac{1}{8 \sqrt{1+z^{2}}}-\frac{5}{24\left(1+z^{2}\right)^{\frac{3}{2}}}, \mathrm{e}$

$$
I_{\nu}^{\prime}(\nu z)=\frac{\left(1+z^{2}\right)^{\frac{1}{4}} \mathrm{e}^{\nu \sqrt{1+z^{2}}} \mathrm{e}^{\nu \log \frac{z}{1+\sqrt{1+z^{2}}}}}{\sqrt{2 \pi \nu} z}\left(1+V_{1}(z) \frac{1}{\nu}+O\left(\nu^{-2}\right)\right)
$$


com $V_{1}(z)=-\frac{3}{8 \sqrt{1+z^{2}}}+\frac{7}{24\left(1+z^{2}\right)^{\frac{3}{2}}}$. Usando a expansão clássica para o logaritmo da função Gamma de Euler, [21] 8.344, obtemos, para $n$ grande, uniformemente em $\lambda$,

$$
\begin{aligned}
\log \Gamma\left(-\lambda, S_{n} / u_{n}^{2}\right)= & \sum_{h=0}^{\infty} \phi_{h-1}(\lambda) u_{n}^{1-h} \\
= & (1-\log 2-\sqrt{1-\lambda}+\log (1+\sqrt{1-\lambda})) \nu n \\
& +\frac{1}{4} \log (1-\lambda)-\left(U_{1}(\sqrt{-\lambda})+\frac{1}{12}\right) \frac{1}{\nu n}+O\left(\frac{1}{(\nu n)^{2}}\right), \\
\log \Gamma\left(-\lambda, \hat{S}_{n} / u_{n}^{2}\right)= & \sum_{h=0}^{\infty} \hat{\phi}_{h-1}(\lambda) u_{n}^{1-h} \\
= & (1-\log 2-\sqrt{1-\lambda}+\log (1+\sqrt{1-\lambda})) \nu n \\
& -\frac{1}{4} \log (1-\lambda)-\left(V_{1}(\sqrt{-\lambda})+\frac{1}{12}\right) \frac{1}{\nu n}+O\left(\frac{1}{(\nu n)^{2}}\right),
\end{aligned}
$$

em particular

$$
\begin{aligned}
& \phi_{1}(\lambda)=-\frac{1}{8} \frac{1}{(1-\lambda)^{\frac{1}{2}}}+\frac{5}{24} \frac{1}{(1-\lambda)^{\frac{3}{2}}}-\frac{1}{12} \\
& \hat{\phi}_{1}(\lambda)=\frac{3}{8} \frac{1}{(1-\lambda)^{\frac{1}{2}}}-\frac{7}{24} \frac{1}{(1-\lambda)^{\frac{3}{2}}}-\frac{1}{12} .
\end{aligned}
$$

Note que o comprimento $\ell$ da decomposição é precisamente 2 , pois e $(U)=1$ e, portanto, o maior inteiro tal que $h-1=\sigma_{h} \leq 1$ é 2 , como $\sigma_{0}=-1, \sigma_{1}=0, \sigma_{2}=1$. Entretanto, pelo teorema 4, apenas o termo $\operatorname{com} \sigma_{h}=1$, assim $h=2$, aparece na fórmula do teorema 5 , como o único polo de $\zeta(s, U)$ é em $s=1$. Aplicaremos a fórmula do teorema 5. Primeiro, como

$$
\operatorname{Res}_{s=1} \zeta(s, U)=\frac{1}{\nu}(\gamma+\log \nu), \quad \operatorname{Res}_{s=1} \zeta(s, U)=\frac{1}{\nu},
$$

onde $\gamma$ é a constante de Euler. Segue que

$$
\begin{aligned}
\zeta(0, S) & -\zeta(0, \hat{S})=-A_{0,1}(0)+\hat{A}_{0,1}(0)+\frac{1}{2 \nu} \operatorname{Res}_{s=0}\left(\Phi_{1}(s)-\hat{\Phi}_{1}(s)\right) \\
\zeta^{\prime}(0, S) & -\zeta^{\prime}(0, \hat{S})=-A_{0,0}(0)-A_{0,1}^{\prime}(0)+\hat{A}_{0,0}(0)+\hat{A}_{0,1}^{\prime}(0) \\
& +\frac{1}{2 \nu} \operatorname{Res}_{s=0}\left(\Phi_{1}(s)-\hat{\Phi}_{1}(s)\right)+\frac{1}{\nu}\left(\frac{3}{2} \gamma+\log \nu\right) \operatorname{Res}_{s=0}\left(\Phi_{1}(s)-\hat{\Phi}_{1}(s)\right)
\end{aligned}
$$

Segundo, pela definição na equação (1.19),

$$
\begin{aligned}
& \Phi_{1}(s)=\int_{0}^{\infty} t^{s-1} \frac{1}{2 \pi \mathrm{i}} \int_{\Lambda_{\theta, c}} \frac{\mathrm{e}^{-\lambda t}}{-\lambda}\left(-\frac{1}{8} \frac{1}{(1-\lambda)^{\frac{1}{2}}}+\frac{5}{24} \frac{1}{(1-\lambda)^{\frac{3}{2}}}-\frac{1}{12}\right) d \lambda d t, \\
& \hat{\Phi}_{1}(s)=\int_{0}^{\infty} t^{s-1} \frac{1}{2 \pi \mathrm{i}} \int_{\Lambda_{\theta, c}} \frac{\mathrm{e}^{-\lambda t}}{-\lambda}\left(\frac{3}{8} \frac{1}{(1-\lambda)^{\frac{1}{2}}}-\frac{7}{24} \frac{1}{(1-\lambda)^{\frac{3}{2}}}-\frac{1}{12}\right) d \lambda d t .
\end{aligned}
$$


Usando a fórmula (1.23). Obtemos

$$
\Phi_{1}(s)=\frac{\Gamma\left(s+\frac{1}{2}\right)}{12 \sqrt{\pi} s}(1+5 s), \quad \hat{\Phi}_{1}(s)=\frac{\Gamma\left(s+\frac{1}{2}\right)}{12 \sqrt{\pi} s}(1-7 s),
$$

e assim

$$
\begin{array}{ll}
\operatorname{Res}_{s=0} \Phi_{1}(s)=\frac{5-\gamma}{12}-\frac{1}{6} \log 2, & \operatorname{Res}_{s=0} \Phi_{1}(s)=\frac{1}{12}, \\
\underset{s=0}{\operatorname{Res}_{0}} \hat{\Phi}_{1}(s)=-\frac{7+\gamma}{12}-\frac{1}{6} \log 2, & \operatorname{Res}_{s=0} \hat{\Phi}_{1}(s)=\frac{1}{12} .
\end{array}
$$

Logo a equação (4.27), fica

$$
\begin{aligned}
Z(0)-\hat{Z}(0) & =-\left(A_{0,1}(0)-\hat{A}_{0,1}(0)\right) \\
Z^{\prime}(0)-\hat{Z}^{\prime}(0) & =-\left(A_{0,0}(0)+A_{0,1}^{\prime}(0)-\hat{A}_{0,0}(0)-\hat{A}_{0,1}^{\prime}(0)\right)+\frac{1}{2 \nu} .
\end{aligned}
$$

Terceiro, por meio da equação (1.21) e o teorema 4, os termos $A_{0,0}(0)$ e $A_{0,1}^{\prime}(0)$, são

$$
\begin{aligned}
& A_{0,0}(s)=\sum_{n=1}^{\infty}\left(a_{0,0, n}-b_{1,0,0} u_{n}^{-1}\right) u_{n}^{-2 s}, \\
& A_{0,1}(s)=\sum_{n=1}^{\infty}\left(a_{0,1, n}-b_{1,0,1} u_{n}^{-1}\right) u_{n}^{-2 s} .
\end{aligned}
$$

Assim, precisamos da expansão, para $\lambda$ grande, das funções $\log \Gamma\left(-\lambda, S_{n} / u_{n}^{2}\right)$ e $\phi_{1}(\lambda)$. Usando expansões clássicas para as funções de Bessel e sua derivada,

$$
I_{\nu}(z) \sim \frac{\mathrm{e}^{z}}{\sqrt{2 \pi z}} \sum_{k=0}^{\infty} c_{k} z^{-k}+O\left(\mathrm{e}^{-z}\right), \quad I_{\nu}^{\prime}(z) \sim \frac{\mathrm{e}^{z}}{\sqrt{2 \pi z}} \sum_{k=0}^{\infty} \hat{c}_{k} z^{-k}+O\left(\mathrm{e}^{-z}\right),
$$

onde $c_{0}=\hat{c}_{0}=1, c_{k}=\frac{(-1)^{k} \Gamma(\nu+k+1 / 2)}{2^{k} k ! \Gamma(\nu-k+1 / 2)}$, e os $\hat{c}_{k}$ são combinações finitas dos $c_{k}$ e as fórmulas na equação (1.20), obtemos

$$
\begin{aligned}
& a_{0,0, n}=\frac{1}{2} \log 2 \pi+\left(\nu n+\frac{1}{2}\right) \log \nu n-\nu n \log 2-\log \Gamma(\nu n+1), \\
& a_{0,1, n}=\frac{1}{2}\left(\nu n+\frac{1}{2}\right), \\
& b_{1,0,0}=-\frac{1}{12}, \quad b_{1,0,1}=0,
\end{aligned}
$$

e

$$
\begin{aligned}
& \hat{a}_{0,0, n}=\frac{1}{2} \log 2 \pi+\left(\nu n+\frac{1}{2}\right) \log \nu n-\nu n \log 2-\log \Gamma(\nu n+1), \\
& \hat{a}_{0,1, n}=\frac{1}{2}\left(\nu n-\frac{1}{2}\right), \\
& \hat{b}_{1,0,0}=-\frac{1}{12}, \quad \hat{b}_{1,0,1}=0 .
\end{aligned}
$$


Isto mostra que $A_{0,0}(0)=\hat{A}_{0,0}(0)$ e que

$$
A_{0,1}(s)-\hat{A}_{0,1}(s)=\frac{1}{2} \sum_{n=1}^{\infty} u_{n}^{-2 s}=\frac{1}{2} \zeta(2 s, U)=\frac{1}{2} \nu^{-2 s} \zeta(2 s)
$$

e assim

$$
A_{0,1}(0)-\hat{A}_{0,1}(0)=-\frac{1}{4}, \quad A_{0,1}^{\prime}(0)-\hat{A}_{0,1}^{\prime}(0)=\frac{1}{2} \log \nu-\frac{1}{2} \log 2 \pi .
$$

Substituindo na equação (4.28) obtemos

$$
\begin{aligned}
Z(0)-\hat{Z}(0) & =\frac{1}{4}, \\
Z^{\prime}(0)-\hat{Z}^{\prime}(0) & =-\frac{1}{2} \log \nu+\frac{1}{2} \log 2 \pi+\frac{1}{2 \nu} .
\end{aligned}
$$

Com isto, em (4.25), obtemos

$$
\log T_{\mathrm{abs}}\left(C_{\alpha} S_{l \operatorname{sen} \alpha}^{1}\right)=\frac{1}{2} \log \frac{\pi}{\nu} l^{2}+\frac{1}{2 \nu} .
$$

Em particular, para $\alpha=\frac{\pi}{2}$, temos que

$$
\log T_{\text {abs }}\left(D_{l}^{2}\right)=-\log T_{\text {rel }}\left(D_{l}^{2}\right)=\frac{1}{2} \log \pi l^{2}+\frac{1}{2} .
$$

\subsubsection{Torção Analítica do cone sobre uma esfera}

Seja

$$
F(s, \nu)=\sum_{n=1}^{\infty}(2 n+1) \mu_{n}^{-2 s}\left(\log \left(1+\frac{1}{2 \mu_{n}}\right)-\log \left(1-\frac{1}{2 \mu_{n}}\right)\right) .
$$

Teorema 16. A Torção Analítica do cone $C_{\alpha} S_{l \operatorname{sen} \alpha}^{2}$ de ângulo $\alpha$ e comprimento $l>0$, sobre a esfera, com a métrica padrão $g_{E}$ induzida pela imersão, com condições de contorno absoluta (relativa), e uma representação ortogonal $\rho$ do grupo fundamental de posto 1 é:

$$
\log T_{\text {abs }}\left(C_{\alpha} S_{l \operatorname{sen} \alpha}^{2}\right)=\log T_{\text {rel }}\left(C_{\alpha} S_{l \operatorname{sen} \alpha}^{2}\right)=\frac{1}{2} \log \frac{4}{3} l^{3}-\frac{1}{2} F(0, \csc \alpha)+\frac{1}{4} \operatorname{sen}^{2} \alpha,
$$

onde a função $F(s, \nu)$ é dada como anteriormente. Em particular, para o disco $D_{l}^{3}$ temos:

$$
\log T_{\mathrm{abs}}\left(D_{l}^{3}\right)=\log T_{\mathrm{rel}}\left(D_{l}^{3}\right)=\frac{1}{2} \log \frac{4 \pi l^{3}}{3}+\frac{1}{2} \log 2+\frac{1}{4}
$$

Demonstração. Claramente a Torção Analítica com as condições de contorno absoluta e relativa coincidem neste caso, basta ver os autovalores. Consideramos o caso com condições de 
contorno absoluta no bordo. Usando a seção 4.3.1, as funções zeta relevantes são

$$
\begin{aligned}
& \zeta\left(s, \Delta^{(1)}\right)=\sum_{k=1}^{\infty} \frac{j_{\frac{3}{2}, k}^{-2 s}}{l^{-2 s}}+\sum_{n, k=1}^{\infty}(2 n+1) \frac{j_{\mu_{n}, k}^{-2 s}}{l^{-2 s}}+\sum_{n, k=1}^{\infty}(2 n+1) \frac{\tilde{j}_{\mu_{n}, k, \pm}^{-2 s}}{l^{-2 s}} \\
& \zeta\left(s, \Delta^{(2)}\right)=\sum_{k=1}^{\infty} \frac{j_{\frac{1}{2}, k}^{-2 s}}{l^{-2 s}}+2 \sum_{n, k=1}^{\infty}(2 n+1) \frac{j_{\mu_{n}, k}^{-2 s}}{l^{-2 s}}+\sum_{n, k=1}^{\infty}(2 n+1) \frac{\tilde{j}_{\mu_{n}, k,+}^{-2 s}}{l^{-2 s}} \\
& \zeta\left(s, \Delta^{(3)}\right)=\sum_{k=1}^{\infty} \frac{j_{\frac{1}{2}, k}^{-2 s}}{l^{-2 s}}+\sum_{n, k=1}^{\infty}(2 n+1) \frac{j_{\mu_{n}, k}^{-2 s}}{l^{-2 s}}
\end{aligned}
$$

e pela equação (2.11), a torção é

$$
\log T_{\mathrm{abs}}\left(\left(C_{\alpha} S_{l \alpha}^{2}, g_{E}\right), \rho\right)=-\frac{1}{2} \zeta^{\prime}\left(0, \Delta^{(1)}\right)+\zeta^{\prime}\left(0, \Delta^{(2)}\right)-\frac{3}{2} \zeta^{\prime}\left(0, \Delta^{(3)}\right) .
$$

Defina a função

$$
\begin{aligned}
t(s) & =-\frac{1}{2} \zeta\left(s, \Delta^{(1)}\right)+\zeta\left(s, \Delta^{(2)}\right)-\frac{3}{2} \zeta\left(s, \Delta^{(3)}\right) \\
& =-\frac{1}{2} \sum_{k=1}^{\infty} \frac{j_{\frac{1}{2}, k}^{-2 s}}{l^{-2 s}}-\frac{1}{2} \sum_{k=1}^{\infty} \frac{j_{\frac{3}{2}, k}^{-2 s}}{l^{-2 s}}+\frac{1}{2} \sum_{n, k=1}^{\infty}(2 n+1) \frac{\tilde{j}_{\mu_{n}, k,+}^{-2 s}}{l^{-2 s}}-\frac{1}{2} \sum_{n, k=1}^{\infty}(2 n+1) \frac{\tilde{j}_{\mu_{n}, k,-}^{-2 s}}{l^{-2 s}} \\
& =l^{2 s}\left(-\frac{1}{2} z_{\frac{1}{2}}(s)-\frac{1}{2} z_{\frac{3}{2}}(s)+\frac{1}{2} Z_{+}(s)-\frac{1}{2} Z_{-}(s)\right),
\end{aligned}
$$

então

$$
\begin{aligned}
\log T_{\mathrm{abs}}\left(C_{\alpha} S_{l \alpha}^{2}\right)=t^{\prime}(0)=\log l^{2} & \left(-\frac{1}{2} z_{\frac{1}{2}}(0)-\frac{1}{2} z_{\frac{3}{2}}(0)+\frac{1}{2} Z_{+}(0)-\frac{1}{2} Z_{-}(0)\right) \\
& -\frac{1}{2} z_{\frac{1}{2}}^{\prime}(0)-\frac{1}{2} z_{\frac{3}{2}}^{\prime}(0)+\frac{1}{2} Z_{+}^{\prime}(0)-\frac{1}{2} Z_{-}^{\prime}(0) .
\end{aligned}
$$

Usando as equações (1.22) da seção 1.5, obtemos

$$
\log T_{\mathrm{abs}}\left(C_{\alpha} S_{l \alpha}^{2}\right)=\left(\frac{3}{4}+\frac{1}{2} Z_{+}(0)-\frac{1}{2} Z_{-}(0)\right) \log l^{2}+\frac{1}{2} Z_{+}^{\prime}(0)-\frac{1}{2} Z_{-}^{\prime}(0)+\frac{1}{2} \log \frac{4}{3} .
$$

Falta estudar as diferenças $Z_{+}(0)-Z_{-}(0)$ e $Z_{+}^{\prime}(0)-Z_{-}^{\prime}(0)$. Para isto usaremos o teorema [5, na forma apresentada no seu corolário. As seqüências relevantes são as seqüências duplas $S_{ \pm}=\left\{\tilde{j}_{\mu_{n}, k, \pm}^{2}\right\}$ e a seqüência simples $U=\left\{(2 n+1): \mu_{n}\right\}_{n=1}^{\infty}$, onde

$$
\mu_{n}=\sqrt{\nu^{2} n(n+1)+\frac{1}{4}}
$$

e $Z_{ \pm}(s)=\zeta\left(s, S_{ \pm}\right)$. Usando estimativas clássicas para os zeros das funções de Bessel [48], o genus de $S_{ \pm}$é 0 , o genus de $U$ é 2 , e os genus relativos de $S_{ \pm}$são $(1,1,0)$. Isto difere apenas do caso do círculo por $\mathrm{g}\left(S_{ \pm, k}\right)$, com $k$ fixo. Usando estimativas clássicas para os zeros das funções de Bessel, o comportamento destas seqüências é dado pelo comportamento da seqüência dos autovalores do Laplaciano sobre a esfera $S^{2}$, que é conhecido. Em particular lembramos os 
principais fatos mais a frente. Verificamos que $U$ e $S_{ \pm, n}$ são seqüências totalmente regulares do tipo espectral.

Por definição da seqüência $U$,

$$
\zeta(s, U)=\nu^{-s} \zeta\left(s, L_{\frac{1}{4 \nu^{2}}}\right),
$$

onde $L_{q}=\{(2 n+1): \sqrt{n(n+1)+q}\}_{n=1}^{\infty}$. Assim, $U$ está relacionado com a seqüência de autovalores do Laplaciano sobre a 2-esfera transladado por uma constante positiva $q$. Mais precisamente $\zeta\left(2 s, L_{0}\right)=\zeta\left(s, \operatorname{Sp}_{+} \Delta_{S^{2}}^{(0)}\right)$. A função zeta $\zeta\left(s, \operatorname{Sp}_{+} \Delta_{S^{2}}^{(0)}\right)$ foi estudada em [43], seção 3.3, onde foi provado que e $\left(\operatorname{Sp}_{+} \Delta_{S^{2}}^{(0)}\right)=\mathrm{g}\left(\operatorname{Sp}_{+} \Delta_{S^{2}}^{(0)}\right)=1$ e que $\operatorname{Sp}_{+} \Delta_{S^{2}}^{(0)}$ é uma seqüência totalmente regular do tipo espectral com ordem infinita, por meio da apresentação explicita para a fórmula da função Gamma associada $\Gamma(-\lambda, U)$ em termos da Função $G$ de Barnes. Segue que e $(U)=\mathrm{g}(U)=2$ e que $U$ é uma seqüência totalmente regular do tipo espectral com ordem infinita. Ainda $\zeta\left(s, \operatorname{Sp}_{+} \Delta_{S^{2}}^{(0)}\right)$ possui um polo simples em $s=1$, com resíduos

$$
\operatorname{Res}_{s=1} \zeta\left(s, \operatorname{Sp}_{+} \Delta_{S^{2}}^{(0)}\right)=2 \gamma, \quad \operatorname{Res}_{s=1} \zeta\left(s, \operatorname{Sp}_{+} \Delta_{S^{2}}^{(0)}\right)=1,
$$

e assim, $\zeta\left(s, L_{0}\right)$ possui um polo simples em $s=2$, com a mesma parte finita e resíduo duplicado. Expandindo a potência do binômio, temos que

$$
\zeta\left(s, L_{q}\right)=\zeta\left(s, L_{0}\right)+f(s),
$$

onde $f(s)$ é regular em $s=2$. Portanto,

$$
\operatorname{Res}_{s=2} \zeta\left(s, L_{q}\right)=2 \gamma+f(2), \quad \operatorname{Res}_{s=2} \zeta\left(s, L_{q}\right)=2,
$$

$\mathrm{e}$

$$
\zeta(s, U)=\nu^{-s} \zeta\left(s, L_{q}\right)=\frac{2}{\nu^{2}} \frac{1}{s-2}+f(s),
$$

próximo a $s=2$, onde $f(s)$ é alguma função regular. Para $S_{ \pm}$, procederemos como segue. Defina a função

$$
G_{\nu}^{ \pm}(z)= \pm \frac{1}{2} J_{\nu}(z)+z J_{\nu}^{\prime}(z)
$$

Lembrando a definição da função de Bessel em série

$$
J_{\nu}(z)=\frac{z^{\nu}}{2^{\nu}} \sum_{k=0}^{\infty} \frac{(-1)^{k} z^{2 k}}{2^{2 k} k ! \Gamma(\nu+k+1)},
$$

obtemos próximo a $z=0$

$$
G_{\nu}^{ \pm}(z)=\left(1 \pm \frac{1}{2 \nu}\right) \frac{z^{\nu}}{2^{\nu} \Gamma(\nu)}
$$

Isto significa que a função $\hat{G}_{\nu}^{ \pm}(z)=z^{-\nu} G_{\nu}^{ \pm}(z)$ é uma função par em $z$. Sejam $z_{\nu, k, \pm}$ os zeros positivos de $G_{\nu}^{ \pm}(z)$ ordenados em ordem crescente. Pelo teorema da fatorização de Hadamard [12, temos a expansão em produto

$$
\hat{G}_{\nu}^{ \pm}(z)=\hat{G}_{\nu}^{ \pm}(z) \prod_{k=-\infty}^{+\infty}\left(1-\frac{z}{z_{\nu, k, \pm}}\right),
$$


e portanto

$$
G_{\nu}^{ \pm}(z)=\left(1 \pm \frac{1}{2 \nu}\right) \frac{z^{\nu}}{2^{\nu} \Gamma(\nu)} \prod_{k=1}^{\infty}\left(1-\frac{z^{2}}{z_{\nu, k, \pm}^{2}}\right) .
$$

Agora, lembrando que (quando $-\pi<\arg (z)<\frac{\pi}{2}$ )

$$
J_{\nu}(\mathrm{i} z)=\mathrm{e}^{\frac{\pi}{2} \mathrm{i} \nu} I_{\nu}(z), J_{\nu}^{\prime}(\mathrm{i} z)=\mathrm{e}^{\frac{\pi}{2} \mathrm{i} \nu} \mathrm{e}^{-\frac{\pi}{2} \mathrm{i}} I_{\nu}^{\prime}(z),
$$

obtemos

$$
G_{\nu}^{ \pm}(\mathrm{i} z)=\mathrm{e}^{\frac{\pi}{2} \mathrm{i} \nu}\left( \pm \frac{1}{2} I_{\nu}(z)+z I_{\nu}^{\prime}(z)\right) .
$$

Então, definimos (para $\left.-\pi<\arg (z)<\frac{\pi}{2}\right)$

$$
H_{\nu}^{ \pm}(z)=\mathrm{e}^{-\frac{\pi}{2} \mathrm{i} \nu} G_{\nu}^{ \pm}(\mathrm{i} z)
$$

e assim

$$
H_{\nu}^{ \pm}(z)= \pm \frac{1}{2} I_{\nu}(z)+z I_{\nu}^{\prime}(z)=\left(1 \pm \frac{1}{2 \nu}\right) \frac{z^{\nu}}{2^{\nu} \Gamma(\nu)} \prod_{k=1}^{\infty}\left(1+\frac{z^{2}}{z_{\nu, k, \pm}^{2}}\right)
$$

Usando estas representações, obtemos a representação a seguir para a função Gamma associada a seqüência $S_{ \pm, n}$. Pela definição na equação (1.17), com $z=\sqrt{-\lambda}$, temos

$$
\begin{aligned}
\log \Gamma\left(-\lambda, S_{ \pm, n}\right) & =-\log \prod_{k=1}^{\infty}\left(1+\frac{(-\lambda)}{\tilde{j}_{\mu_{n}, k, \pm}^{2}}\right) \\
& =-\log H_{\mu_{n}}^{ \pm}(\sqrt{-\lambda})+\mu_{n} \log \sqrt{-\lambda}-\log 2^{\mu_{n}} \Gamma\left(\mu_{n}\right)+\log \left(1 \pm \frac{1}{2 \mu_{n}}\right)
\end{aligned}
$$

A primeira conseqüência desta representação é que temos uma expansão assintótica completa para a função Gamma $\log \Gamma\left(-\lambda, S_{ \pm, n}\right)$, e portanto $S_{n}$ e $\hat{S}_{n}$ são seqüências do tipo espectral. Considerando as expansões, segue que ambas são seqüências totalmente regular do tipo espectral com ordem infinita.

A seguir, provamos que $S_{ \pm}$são espectralmente decompostas sobre $U$ com potência $\kappa=2$ e comprimento $\ell=3$. Temos que mostrar que as funções $\log \Gamma\left(-\lambda, S_{ \pm, n} / u_{n}^{2}\right)$ possuem a expansão uniforme apropriada para $n$ grande. Temos

$$
\begin{aligned}
\log \Gamma\left(-\lambda, S_{ \pm, n} / \mu_{n}^{2}\right)= & -\log H_{\mu_{n}}^{ \pm}\left(\mu_{n} \sqrt{-\lambda}\right)+\mu_{n} \log \sqrt{-\lambda}+\mu_{n} \log \mu_{n} \\
& -\mu_{n} \log 2-\log \Gamma\left(\mu_{n}\right)+\log \left(1 \pm \frac{1}{2 \mu_{n}}\right) .
\end{aligned}
$$

Lembrando as expansões dadas na seção 4.3.1, obtemos

$$
H_{\nu}^{ \pm}(\nu z)=\sqrt{\nu}\left(1+z^{2}\right)^{\frac{1}{4}} \frac{\mathrm{e}^{\nu \sqrt{1+z^{2}}} \mathrm{e}^{\nu \log \frac{z}{1+\sqrt{1+z^{2}}}}}{\sqrt{2 \pi}}\left(1+W_{1, \pm}(z) \frac{1}{\nu}+W_{2, \pm}(z) \frac{1}{\nu^{2}}+O\left(\nu^{-3}\right)\right),
$$

onde

$$
W_{1, \pm}(p)=V_{1}(p) \pm \frac{1}{2} p, \quad W_{2, \pm}(p)=V_{2}(p) \pm \frac{1}{2} p U_{1}(p),
$$


$\operatorname{com} p=\frac{1}{(1-\lambda)^{\frac{1}{2}}}$ e $z=\sqrt{-\lambda}$. Logo

$$
\begin{array}{ll}
W_{1,+}(p)=\frac{1}{8} p+\frac{7}{24} p^{3}, & W_{2,+}(p)=-\frac{7}{128} p^{2}+\frac{79}{192} p^{4}-\frac{455}{1152} p^{6} \\
W_{1,-}(p)=-\frac{7}{8} p+\frac{7}{24} p^{3}, & W_{2,-}(p)=-\frac{28}{128} p^{2}+\frac{119}{192} p^{4}-\frac{455}{1152} p^{6}
\end{array}
$$

Com isto,

$$
\begin{aligned}
\log \Gamma\left(-\lambda, S_{n, \pm} / \mu_{n}^{2}\right)= & \sum_{h=0}^{\infty} \phi_{h-1, \pm}(\lambda) \mu_{n}^{1-h} \\
= & (1-\sqrt{1-\lambda}+\log (1+\sqrt{1-\lambda})-\log 2) \mu_{n} \\
& -\frac{1}{4} \log (1-\lambda)+\left(-W_{1, \pm}(\sqrt{-\lambda}) \pm \frac{1}{2}-\frac{1}{12}\right) \frac{1}{\mu_{n}} \\
& +\left(-W_{2, \pm}(\sqrt{-\lambda})+\frac{1}{2} W_{1, \pm}^{2}(\sqrt{-\lambda})-\frac{1}{8}\right) \frac{1}{\mu_{n}^{2}}+O\left(\frac{1}{\mu_{n}^{3}}\right)
\end{aligned}
$$

e assim

$$
\begin{gathered}
\phi_{1,+}(\lambda)=-\frac{1}{8} \frac{1}{(1-\lambda)^{\frac{1}{2}}}-\frac{7}{24} \frac{1}{(1-\lambda)^{\frac{3}{2}}}+\frac{5}{12}, \\
\phi_{1,-}(\lambda)=\frac{7}{8} \frac{1}{(1-\lambda)^{\frac{1}{2}}}-\frac{7}{24} \frac{1}{(1-\lambda)^{\frac{3}{2}}}-\frac{7}{12}, \\
\phi_{2,+}(\lambda)=\frac{1}{16} \frac{1}{1-\lambda}-\frac{3}{8} \frac{1}{(1-\lambda)^{2}}+\frac{7}{16} \frac{1}{(1-\lambda)^{3}}-\frac{1}{8}, \\
\phi_{2,-}(\lambda)=\frac{9}{16} \frac{1}{1-\lambda}-\frac{7}{8} \frac{1}{(1-\lambda)^{2}}+\frac{7}{16} \frac{1}{(1-\lambda)^{3}}-\frac{1}{8} .
\end{gathered}
$$

O comprimento $\ell$ da decomposição é precisamente 3 , de fato, como $\mathrm{e}(U)=2$, o maior inteiro $h$ tal que $h-1=\sigma_{h} \leq 2$ é 3 , já que $\sigma_{0}=-1, \sigma_{1}=0, \sigma_{2}=1, \sigma_{3}=2$. No entanto, pelo teorema 4, apenas o termo com índice $\sigma_{h}=2$, a saber $h=3$, aparece na fórmula do teorema 5, já que o único polo de $\zeta(s, U)$ é em $s=2$.

Agora aplicaremos a fórmula do teorema 5. Primeiro, como $\kappa=2, \operatorname{Res}_{0_{s=2}} \zeta(s, U)=K$, e $\operatorname{Res}_{1 s=2} \zeta(s, U)=\frac{2}{\nu^{2}}$, obtemos

$$
\begin{aligned}
\zeta\left(0, S_{+}\right)-\zeta\left(0, S_{-}\right)= & -A_{0,1,+}(0)+A_{0,1,-}(0)+\frac{1}{\nu^{2}} \operatorname{Res}_{s=0}\left(\Phi_{2,+}(s)-\Phi_{2,-}(s)\right) \\
\zeta^{\prime}\left(0, S_{+}\right)-\zeta^{\prime}\left(s, S_{-}\right)= & -\left(A_{0,0,+}(0)+A_{0,1,+}^{\prime}(0)-A_{0,0,-}(0)-A_{0,1,-}^{\prime}(0)\right) \\
& +\frac{1}{\nu^{2}} \operatorname{Res}_{s=0}\left(\Phi_{2,+}(s)-\Phi_{2,-}(s)\right)+\frac{K}{2} \operatorname{Res}_{s=0}\left(\Phi_{2,+}(s)-\Phi_{2,-}(s)\right) .
\end{aligned}
$$

Segundo, pela equação (4.36),

$$
\phi_{2,+}(\lambda)-\phi_{2,-}(\lambda)=-\frac{1}{2}\left(\frac{1}{1-\lambda}-\frac{1}{(1-\lambda)^{2}}\right),
$$


e assim, usando a definição da equação (1.19),

$$
\Phi_{2,+}(s)-\Phi_{2,-}(s)=-\frac{1}{2} \int_{0}^{\infty} t^{s-1} \frac{1}{2 \pi i} \int_{\Lambda_{\theta, c}} \frac{\mathrm{e}^{-\lambda t}}{-\lambda}\left(\frac{1}{1-\lambda}-\frac{1}{(1-\lambda)^{2}}\right) .
$$

Com a fórmula (1.23), obtemos

$$
\Phi_{2,+}(s)-\Phi_{2,-}(s)=\frac{1}{2} \Gamma(s+1)
$$

e assim

$$
\operatorname{Res}_{s=0}\left(\Phi_{2,+}(s)-\Phi_{2,-}(s)\right)=\frac{1}{2}, \quad \operatorname{Res}_{s=0}\left(\Phi_{2,+}(s)-\Phi_{2,-}(s)\right)=0 .
$$

Temos

$$
\begin{aligned}
Z_{+}(0)-Z_{-}(0) & =-A_{0,1,+}(0)+A_{0,1,-}(0) \\
Z_{+}^{\prime}(0)-Z_{-}^{\prime}(0) & =\zeta^{\prime}\left(0, S_{+}\right)-\zeta^{\prime}\left(s, S_{-}\right) \\
= & -\left(A_{0,0,+}(0)+A_{0,1,+}^{\prime}(0)-A_{0,0,-}(0)-A_{0,1,-}^{\prime}(0)\right)+\frac{1}{2 \nu^{2}} .
\end{aligned}
$$

Terceiro, pela equação (1.21) e o teorema 4, os termos $A_{0,0}(s)$ e $A_{0,1}(s)$, são

$$
\begin{aligned}
& A_{0,0, \pm}(s)=\sum_{n=1}^{\infty}\left(a_{0,0, n, \pm}-b_{1,0,0, \pm} u_{n}^{-1}\right) u_{n}^{-2 s} \\
& A_{0,1, \pm}(s)=\sum_{n=1}^{\infty}\left(a_{0,1, n, \pm}-b_{1,0,1, \pm} u_{n}^{-1}\right) u_{n}^{-2 s} .
\end{aligned}
$$

Assim, precisamos da expansão para $\lambda$ grande das funções $\log \Gamma\left(-\lambda, S_{n, \pm} / u_{n}^{2}\right)$ e $\phi_{2, \pm}(\lambda)$. Usando as equações (4.29), (4.35) e a definição, obtemos

$$
H_{\nu}^{ \pm}(z) \sim \frac{\sqrt{z} \mathrm{e}^{z}}{\sqrt{2 \pi}}\left(1+\sum_{k=1}^{\infty} b_{k} z^{-k}\right)+O\left(\mathrm{e}^{-z}\right),
$$

para $z$ grande. Portanto,

$$
\begin{aligned}
\log \Gamma\left(-\lambda, S_{n, \pm} / \mu_{n}^{2}\right)= & -\mu_{n} \sqrt{-\lambda}+\frac{1}{2}\left(\mu_{n}-\frac{1}{2}\right) \log (-\lambda)+\frac{1}{2} \log 2 \pi \\
& +\left(\mu_{n}-\frac{1}{2}\right) \log \mu_{n}-\log 2^{\mu_{n}} \Gamma\left(\mu_{n}\right)+\log \left(1 \pm \frac{1}{2 \mu_{n}}\right)+O\left(\frac{1}{\sqrt{-\lambda}}\right) .
\end{aligned}
$$

Ainda, da equação (4.36),

$$
\phi_{2, \pm}(\lambda)=-\frac{1}{8}+O\left(\frac{1}{-\lambda}\right)
$$

Então,

$$
\begin{aligned}
& a_{0,0, n, \pm}=\frac{1}{2} \log 2 \pi+\left(\mu_{n}-\frac{1}{2}\right) \log \mu_{n}-\log 2^{\mu_{n}} \Gamma\left(\mu_{n}\right)+\log \left(1 \pm \frac{1}{2 \mu_{n}}\right), \\
& a_{0,1, n, \pm}=\frac{1}{2}\left(\mu_{n}-\frac{1}{2}\right) \\
& b_{2,0,0, \pm}=-\frac{1}{8}, \quad b_{2,0,1, \pm}=0 .
\end{aligned}
$$


Isto mostra imediatamente que $A_{0,1,+}(s)=A_{0,1,-}(s)$, e portanto $Z_{+}(0)-Z_{-}(0)=0$. Desta forma,

$$
\begin{aligned}
A_{0,0,+}(s)-A_{0,0,-}(s) & =\sum_{n=1}^{\infty}(2 n+1) \mu_{n}^{-2 s}\left(\log \left(1+\frac{1}{2 \mu_{n}}\right)-\log \left(1-\frac{1}{2 \mu_{n}}\right)\right) \\
& =F(s, \nu) .
\end{aligned}
$$

Note que esta série converge uniformemente para a parte real de $s$ maior que 2, mas usando a extensão analítica da função zeta $\zeta(s, U)$, esta série possui uma extensão analítica que é regular em $s=0$. Substituindo em (4.37) temos

$$
Z_{+}^{\prime}(0)-Z_{-}^{\prime}(0)=-F(0, \nu)+\frac{1}{2 \nu^{2}} .
$$

Substituindo em (4.32) obtemos

$$
\log T\left(C_{\alpha} S_{l \operatorname{sen} \alpha}^{2}\right)=\frac{1}{2} \log \frac{4 l^{3}}{3}-\frac{1}{2} F(0, \csc \alpha)+\frac{1}{4} \operatorname{sen}^{2} \alpha .
$$

Consideremos o caso do disco, onde $\nu=1$. Observe que, neste caso, $\mu_{n}=n+\frac{1}{2}$, e portanto

$$
F(s, 1)=2^{2 s} \sum_{n=1}^{\infty}(2 n+1)^{1-2 s}(\log (n+1)-\log n) .
$$

Para $\operatorname{Re}(s)>2$, devido a convergência absoluta, podemos reorganizar os termos da soma. Obtemos

$$
\begin{aligned}
F(s, 1) & =-2^{2 s} \sum_{n=1}^{\infty}\left((2 n+1)^{1-2 s}-(2 n-1)^{1-2 s}\right) \log n \\
& =2 \sum_{j=0}^{\infty}\left(\begin{array}{c}
1-2 s \\
j
\end{array}\right) \frac{\left(1-(-1)^{j}\right)}{2^{j}} \zeta_{R}^{\prime}(2 s+j-1) \\
& =2(1-2 s) \zeta_{R}^{\prime}(2 s)+2 \sum_{k=1}^{\infty}\left(\begin{array}{c}
1-2 s \\
2 k+1
\end{array}\right) \frac{\zeta_{R}^{\prime}(2 s+2 k)}{2^{2 k+1}}
\end{aligned}
$$

e assim, $F(0,1)=2 \zeta_{R}^{\prime}(0)=-\log 2 \pi$. Substituindo na equação (4.38),

$$
\log T\left(C_{\frac{\pi}{2}} S_{l}^{2}\right)=\log T\left(D_{l}^{3}\right)=\frac{1}{2} \log \frac{4 \pi l^{3}}{3}+\frac{1}{2} \log 2+\frac{1}{4} .
$$


4. O disco $m$-dimensional 


\section{Capítulo 5}

\section{A conjectura para esferas de dimensão ímpar}

O objetivo deste capítulo é apresentar uma contribuição, no aspecto quantitativo, para o problema de estender o Teorema de Cheeger Müller no caso de espaços com singularidades do tipo cônico. Neste sentido, determinamos a Torção Analítica do cone sobre a esfera $S^{3}$. O resultado cobre o caso do disco e, portanto, mostra uma contribuição para a discussão para a extensão do Teorema de Cheeger Müller para variedades suaves com bordo, a saber, o problema de estabelecer o termo anômalo correto. Depois, usando o teorema 10, de uma maneira formal, faremos uma conjectura sobre a Torção Analítica do cone sobre as esferas de dimensão ímpar. Este capítulo faz parte do artigo [24].

\subsection{Dimensão Baixa}

Nesta seção determinaremos a Torção Analítica do cone $C_{\alpha} S_{l \operatorname{sen} \alpha}^{3}$. Este é um caso interessante que nos permite ter uma idéia de como fazer para generalizar o resultado para $C_{\alpha} S_{l \operatorname{sen} \alpha}^{2 p-1}$. O processo é basicamente o mesmo dos casos $C_{\alpha} S_{l \operatorname{sen} \alpha}^{1}$ e $C_{\alpha} S_{l \operatorname{sen} \alpha}^{2}$, só que em $C_{\alpha} S_{l \operatorname{sen} \alpha}^{3}$ novos termos aparecem. Isto mostra o quanto que subir a dimensão da base dificulta a solução do problema.

Precisamos do espectro do Laplaciano sobre as formas para $C_{\alpha} S_{l \operatorname{sen} \alpha}^{3}$. Não utilizaremos o método dos lemas 7 e 8. Neste, faremos uso do teorema 3 e, como estaremos interessados apenas no caso absoluto (o caso relativo segue da mesma forma e a única diferença é o sinal), as condições de contorno da equação (2.6). Antes lembramos alguns resultados a respeito dos autovalores do Laplaciano na esfera $S^{3}$.

Em [23], Ikeda e Taniguchi exibem todo o espectro e seus respectivos autoespaço para $S^{n}$, para todo $n$. Precisamos apenas das autoformas coexatas para $S^{3}$. Temos a tabela 


\begin{tabular}{|c|c|c|}
\hline Dimensão & Autovalor & Multiplicidade \\
\hline \hline 0 & $n(n+2)$ & $(n+1)^{2}$ \\
\hline 1 & $(n+1)^{2}$ & $2 n(n+2)$ \\
\hline 2 & $n(n+2)$ & $(n+1)^{2}$ \\
\hline
\end{tabular}

Tabela 5.1: Autovalores das autoformas coexatas da $S^{3}$

Usando os autovalores desta tabela, podemos provar o seguinte lema.

Lema 9. O espectro do operador Laplaciano $\Delta_{C_{\alpha} S_{l \operatorname{sen} \alpha}^{3}}^{(q)}$ sobre as q-formas, com condições de contorno absoluta, é:

$$
\begin{aligned}
& \mathrm{Sp} \Delta_{C_{\alpha} S_{l \operatorname{sen} \alpha}^{3}}^{(0)}=\left\{j_{2, k}^{2} / l^{2}\right\}_{k=1}^{\infty} \cup\left\{(n+1)^{2}: \tilde{j}_{\mu_{0, n}, k,-}^{2} / l^{2}\right\}_{n, k=1}^{\infty}, \\
& \operatorname{Sp} \Delta_{C_{\alpha} S_{l \operatorname{sen} \alpha}^{3}}^{(1)}=\left\{j_{2}^{2} / l^{2}\right\}_{k=1}^{\infty} \cup\left\{2 n(n+2):\left(j_{\mu_{1, n}, k}^{\prime}\right)^{2} / l^{2}\right\}_{n, k=1}^{\infty} \\
& \cup\left\{(n+1)^{2}: \tilde{j}_{\mu_{0, n}, k,-}^{2} / l^{2}\right\}_{n, k=1}^{\infty} \cup\left\{(n+1)^{2}: j_{\mu_{0, n}, k}^{2} / l^{2}\right\}_{n, k=1}^{\infty}, \\
& \operatorname{Sp} \Delta_{C_{\alpha} S_{l \operatorname{sen} \alpha}^{3}}^{(2)}=\left\{(n+1)^{2}: \tilde{j}_{\mu_{0, n}, k,+}^{2} / l^{2}\right\}_{n, k=1}^{\infty} \cup\left\{2 n(n+2):\left(j_{\mu_{1, n}, k}^{\prime}\right)^{2} / l^{2}\right\}_{n, k=1}^{\infty} \\
& \cup\left\{2 n(n+2): j_{\mu_{1, n}, k}^{2} / l^{2}\right\}_{n, k=1}^{\infty} \cup\left\{(n+1)^{2}: j_{\mu_{0, n}, k}^{2} / l^{2}\right\}_{n, k=1}^{\infty}, \\
& \mathrm{Sp} \Delta_{C_{\alpha} S_{l \operatorname{sen} \alpha}^{3}}^{(3)}=\left\{j_{1}^{2} / l^{2}\right\}_{k=1}^{\infty} \cup\left\{(n+1)^{2}: \tilde{j}_{\mu_{0, n}, k,+}^{2} / l^{2}\right\}_{n, k=1}^{\infty} \\
& \cup\left\{(n+1)^{2}: j_{\mu_{0, n}, k}^{2} / l^{2}\right\}_{n, k=1}^{\infty} \cup\left\{2 n(n+2): j_{\mu_{1, n}, k}^{2} / l^{2}\right\}_{n, k=1}^{\infty}, \\
& \mathrm{Sp} \Delta_{C_{\alpha} S_{l \operatorname{sen} \alpha}^{3}}^{(4)}=\left\{j_{1, k}^{2} / l^{2}\right\}_{k=1}^{\infty} \cup\left\{(n+1)^{2}: \tilde{j}_{\mu_{0, n}, k}^{2} / l^{2}\right\}_{n, k=1}^{\infty} \text {, }
\end{aligned}
$$

onde

$$
\mu_{0, n}=\sqrt{\nu^{2} n(n+2)+1}, \quad \mu_{1, n}=\nu(n+1),
$$

e onde os $\tilde{j}_{\nu, k, \pm}$ são os zeros da função $T_{\nu}^{ \pm}(z)= \pm J_{\nu}(z)+z J_{\nu}^{\prime}(z)$.

Demonstração. Parametrizamos $C_{\alpha} S_{l \operatorname{sen} \alpha}^{3}$ por

$$
C_{\alpha} S_{l \operatorname{sen} \alpha}^{3}=\left\{\begin{array}{l}
x_{1}=r \operatorname{sen} \alpha \operatorname{sen} \theta_{3} \operatorname{sen} \theta_{2} \cos \theta_{1} \\
x_{2}=r \operatorname{sen} \alpha \operatorname{sen} \theta_{3} \operatorname{sen} \theta_{2} \operatorname{sen} \theta_{1} \\
x_{3}=r \operatorname{sen} \alpha \operatorname{sen} \theta_{3} \cos \theta_{2} \\
x_{4}=r \operatorname{sen} \alpha \cos \theta_{3} \\
x_{5}=r \cos \alpha
\end{array}\right.
$$

onde $\left(r, \theta_{1}, \theta_{2}, \theta_{3}\right) \in[0, l] \times[0,2 \pi] \times[0, \pi] \times[0, \pi], 0<\alpha \leq \pi / 2$ é um número real fixado e $0<a=\operatorname{sen} \alpha \leq 1$. A métrica induzida é (para $r>0$ )

$$
g=d r \otimes d r+\left(a^{2} r^{2} \operatorname{sen}^{2} \theta_{2} \operatorname{sen}^{2} \theta_{3}\right) d \theta_{1} \otimes d \theta_{1}+\left(a^{2} r^{2} \operatorname{sen}^{2} \theta_{3}\right) d \theta_{2} \otimes d \theta_{2}+\left(a^{2} r^{2}\right) d \theta_{3} \otimes d \theta_{3} .
$$


Usando as condições de contorno absoluta nas formas, descrita na equação (2.6), obtemos as equações a seguir.

Para as 0-formas:

$$
\text { abs. : }\left.\partial_{r} \omega\left(r, \theta_{1}, \theta_{2}, \theta_{3}\right)\right|_{r=l}=0 \text {. }
$$

Para as 1-formas:

$$
\text { abs. : }\left\{\begin{array}{l}
\left.\omega_{r}\left(r, \theta_{1}, \theta_{2}, \theta_{3}\right)\right|_{r=l}=0 \\
\left.\partial_{r} \omega_{\theta_{1}}\left(r, \theta_{1}, \theta_{2}, \theta_{3}\right)\right|_{r=l}=0 \\
\left.\partial_{r} \omega_{\theta_{2}}\left(r, \theta_{1}, \theta_{2}, \theta_{3}\right)\right|_{r=l}=0 \\
\left.\partial_{r} \omega_{\theta_{3}}\left(r, \theta_{1}, \theta_{2}, \theta_{3}\right)\right|_{r=l}=0 .
\end{array}\right.
$$

Para as 2-formas, com $i=1,2,3$ :

$$
\text { abs. : }\left\{\begin{array}{l}
\left.\omega_{r \theta_{i}}\left(r, \theta_{1}, \theta_{2}, \theta_{3}\right)\right|_{r=l}=0 \\
\left.\partial_{r} \omega_{\theta_{1} \theta_{2}}\left(r, \theta_{1}, \theta_{2}, \theta_{3}\right)\right|_{r=l}=0 \\
\left.\partial_{r} \omega_{\theta_{1} \theta_{3}}\left(r, \theta_{1}, \theta_{2}, \theta_{3}\right)\right|_{r=l}=0 \\
\left.\partial_{r} \omega_{\theta_{2} \theta_{3}}\left(r, \theta_{1}, \theta_{2}, \theta_{3}\right)\right|_{r=l}=0 .
\end{array}\right.
$$

Para as 3-formas:

$$
\text { abs. : }\left\{\begin{array}{l}
\left.\omega_{r \theta_{1} \theta_{2}}\left(r, \theta_{1}, \theta_{2}, \theta_{3}\right)\right|_{r=l}=0 \\
\left.\omega_{r \theta_{1} \theta_{3}}\left(r, \theta_{1}, \theta_{2}, \theta_{3}\right)\right|_{r=l}=0 \\
\left.\omega_{r \theta_{2} \theta_{3}}\left(r, \theta_{1}, \theta_{2}, \theta_{3}\right)\right|_{r=l}=0 \\
\left.\partial_{r} \omega_{\theta_{1} \theta_{2} \theta_{3}}\left(r, \theta_{1}, \theta_{2}, \theta_{3}\right)\right|_{r=l}=0 .
\end{array}\right.
$$

Para as 4-formas:

$$
\text { abs. : }\left.\omega_{r \theta_{1} \theta_{2} \theta_{3}}\left(r, \theta_{1}, \theta_{2}, \theta_{3}\right)\right|_{r=l}=0 \text {. }
$$

Agora usando os autovalores das autoformas coexatas da $S^{3}$ que estão na tabela 5.1 e a descrição feita no teorema 3 temos que $\mu_{0, n}=\mu_{2, n}=\sqrt{\nu^{2} n(n+2)+1}$ e $\mu_{1, n}=\nu(n+1)$, e as autoformas do Laplaciano de $C_{\alpha} S_{l \operatorname{sen} \alpha}^{3}$ são as que seguem.

Para 0-formas:

$$
\psi_{1, n}^{(0)}=x^{-1} J_{\mu_{0, n}}(\lambda x) \phi_{n}^{(0)}\left(\theta_{1}, \theta_{2}, \theta_{3}\right), \quad \psi_{E}^{(0)}=x^{-1} J_{1}(\lambda x) h^{0}\left(\theta_{1}, \theta_{2}, \theta_{3}\right) .
$$

Para as 1-formas:

$$
\begin{aligned}
& \psi_{1, n}^{(1)}=x^{-1} J_{\mu_{1, n}}(\lambda x) \phi_{n}^{(1)}\left(\theta_{1}, \theta_{2}, \theta_{3}\right), \\
& \psi_{2, n}^{(1)}=x^{-1} J_{\mu_{0, n}}(\lambda x) d \phi_{n}^{(0)}\left(\theta_{1}, \theta_{2}, \theta_{3}\right)+\partial_{x}\left(x^{-1} J_{\mu_{0, n}}(\lambda x)\right) d x \wedge \psi_{n}^{(0)}\left(\theta_{1}, \theta_{2}, \theta_{3}\right), \\
& \psi_{3, n}^{(1)}=x^{-1} \partial_{x}\left(x J_{\mu_{0, n}}(\lambda x)\right) d \phi_{n}^{(0)}\left(\theta_{1}, \theta_{2}, \theta_{3}\right)+x^{-2} J_{\mu_{0, n}}(\lambda x) d x \wedge \tilde{\delta} \tilde{d} \phi_{n}^{(0)}\left(\theta_{1}, \theta_{2}, \theta_{3}\right), \\
& \psi_{O}^{(1)}=\partial_{x}\left(x^{-1} J_{1}(\lambda x)\right) d x \wedge h^{(0)}\left(\theta_{1}, \theta_{2}, \theta_{3}\right)
\end{aligned}
$$


Para as 2-formas:

$$
\begin{aligned}
& \psi_{1, n}^{(2)}=x J_{\mu_{0, n}}(\lambda x) \phi_{n}^{(2)}\left(\theta_{1}, \theta_{2}, \theta_{3}\right), \\
& \psi_{2, n}^{(2)}=J_{\mu_{1, n}}(\lambda x) d \phi_{n}^{(1)}\left(\theta_{1}, \theta_{2}, \theta_{3}\right)+\partial_{x}\left(J_{\mu_{1, n}}(\lambda x)\right) d x \wedge \phi_{n}^{(2)}\left(\theta_{1}, \theta_{2}, \theta_{3}\right), \\
& \psi_{3, n}^{(2)}=x \partial_{x}\left(J_{\mu_{1, n}}(\lambda x)\right) d \phi_{n}^{(1)}\left(\theta_{1}, \theta_{2}, \theta_{3}\right)+x^{-1} J_{\mu_{1, n}}(\lambda x) d x \wedge \tilde{\delta} \tilde{d} \phi_{n}^{(1)}\left(\theta_{1}, \theta_{2}, \theta_{3}\right), \\
& \psi_{4, n}^{(2)}=J_{\mu_{0, n}}(\lambda x) d x \wedge d \phi_{n}^{(0)}\left(\theta_{1}, \theta_{2}, \theta_{3}\right) .
\end{aligned}
$$

Para as 3-formas:

$$
\begin{aligned}
& \psi_{2, n}^{(3)}=x J_{\mu_{0, n}}(\lambda x) d \phi_{n}^{(2)}\left(\theta_{1}, \theta_{2}, \theta_{3}\right)+\partial_{x}\left(x J_{\mu_{0, n}}(\lambda x)\right) d x \wedge \phi_{n}^{(2)}\left(\theta_{1}, \theta_{2}, \theta_{3}\right), \\
& \psi_{3, n}^{(3)}=x^{3} \partial_{x}\left(x^{-1} J_{\mu_{0, n}}(\lambda x)\right) d \phi_{n}^{(2)}\left(\theta_{1}, \theta_{2}, \theta_{3}\right)+J_{\mu_{0, n}}(\lambda x) d x \wedge \tilde{\delta} \tilde{d} \phi_{n}^{(2)}\left(\theta_{1}, \theta_{2}, \theta_{3}\right), \\
& \psi_{4, n}^{(3)}=x J_{\mu_{1, n}}(\lambda x) d x \wedge d \phi_{n}^{(1)}\left(\theta_{1}, \theta_{2}, \theta_{3}\right), \\
& \psi_{E}^{(3)}=x^{2} J_{2}(\lambda x) h^{3}\left(\theta_{1}, \theta_{2}, \theta_{3}\right) .
\end{aligned}
$$

Para as 4-formas:

$$
\psi_{4, n}^{(4)}=x^{2} J_{\mu_{0, n}}(\lambda x) d x \wedge d \phi_{n}^{(2)}\left(\theta_{1}, \theta_{2}, \theta_{3}\right), \quad \psi_{O}^{(4)}=\partial_{x}\left(x^{2} J_{2}(\lambda x)\right) d x \wedge h^{3}\left(\theta_{1}, \theta_{2}, \theta_{3}\right) .
$$

Onde os $\phi_{n}^{(i)}\left(\theta_{1}, \theta_{2}, \theta_{3}\right)$, para $i=0,1,2$, são autoformas coexatas para o Laplaciano na $S^{3}$, e $h^{(0)}\left(\theta_{1}, \theta_{2}, \theta_{3}\right)$, e $h^{(3)}\left(\theta_{1}, \theta_{2}, \theta_{3}\right)$ são formas harmônicas do Laplaciano na $S^{3}$.

Com estas autoformas e as condições de contorno dadas nas equações (5.1), (5.2), (5.3), (5.4) e (5.5), o resultado procede.

Teorema 17. A Torção Analítica do cone $C_{\alpha} S_{l \operatorname{sen} \alpha}^{3}$ de ângulo a e comprimento $l>0$, sobre a três esfera $S^{3}$, com a métrica induzida pela imersão em $\mathbb{R}^{5}$, com condições de contorno absoluta é:

$$
\begin{aligned}
\log T\left(C_{\alpha} S_{l \operatorname{sen} \alpha}^{3}\right) & =\frac{1}{2} \log \operatorname{Vol}\left(C_{\alpha} S_{l \operatorname{sen} \alpha}^{3}\right)+\frac{3}{4} \operatorname{sen} \alpha-\frac{1}{12} \operatorname{sen}^{3} \alpha \\
& =\frac{1}{2} \log \frac{\pi^{2} l^{4} \operatorname{sen}^{3} \alpha}{2}+\frac{3}{4} \operatorname{sen} \alpha-\frac{1}{12} \operatorname{sen}^{3} \alpha .
\end{aligned}
$$

Demonstração. Usando o lema anterior, as funções zeta relevantes para a Torção Analítica são

$$
\begin{aligned}
\zeta\left(s, \Delta^{(1)}\right) & =\sum_{k=1}^{\infty} \frac{j_{2, k}^{-2 s}}{l^{-2 s}}+2 \sum_{n, k=1}^{\infty} n(n+2) \frac{\left(j_{\mu_{1, n}, k}^{\prime}\right)^{-2 s}}{l^{-2 s}}+\sum_{n, k=1}^{\infty}(n+1)^{2} \frac{\tilde{j}_{\mu_{0, n}, k,-}^{-2 s}}{l^{-2 s}} \\
& +\sum_{n, k=1}^{\infty}(n+1)^{2} \frac{j_{\mu_{0, n}, k}^{-2 s}}{l^{-2 s}} \\
\zeta\left(s, \Delta^{(2)}\right) & =\sum_{n, k=1}^{\infty}(n+1)^{2} \frac{\tilde{j}_{\mu_{0, n}, k,+}^{-2 s}}{l^{-2 s}}+2 \sum_{n, k=1}^{\infty} n(n+2) \frac{\left(j_{\mu_{1, n}, k}^{\prime}\right)^{-2 s}}{l^{-2 s}} \\
& +2 \sum_{n, k=1}^{\infty} n(n+2) \frac{j_{\mu_{1, n}, k}^{-2 s}}{l^{-2 s}}+\sum_{n, k=1}^{\infty}(n+1)^{2} \frac{j_{\mu_{0, n}, k}^{-2 s}}{l^{-2 s}},
\end{aligned}
$$




$$
\begin{aligned}
\zeta\left(s, \Delta^{(3)}\right) & =\sum_{k=1}^{\infty} \frac{j_{1, k}^{-2 s}}{l^{-2 s}}+2 \sum_{n, k=1}^{\infty} n(n+2) \frac{j_{\mu_{1, n}, k}^{-2 s}}{l^{-2 s}}+\sum_{n, k=1}^{\infty}(n+1)^{2} \frac{\tilde{j}_{\mu_{0, n}, k,+}^{-2 s}}{l^{-2 s}} \\
& +\sum_{n, k=1}^{\infty}(n+1)^{2} \frac{j_{\mu_{0, n}, k}^{-2 s}}{l^{-2 s}}, \\
\zeta\left(s, \Delta^{(4)}\right) & =\sum_{k=1}^{\infty} \frac{j_{1, k}^{-2 s}}{l^{-2 s}}+\sum_{n, k=1}^{\infty}(n+1)^{2} \frac{j_{\mu_{0, n}, k}^{-2 s}}{l^{-2 s}} .
\end{aligned}
$$

Pela equação (2.11), a torção é $\left(a=\operatorname{sen} \alpha=\frac{1}{\nu}\right)$

$$
\log T\left(C_{\alpha} S_{l a}^{3}\right)=-\frac{1}{2} \zeta^{\prime}\left(0, \Delta^{(1)}\right)+\zeta^{\prime}\left(0, \Delta^{(2)}\right)-\frac{3}{2} \zeta^{\prime}\left(0, \Delta^{(3)}\right)+2 \zeta^{\prime}\left(0, \Delta^{(4)}\right) .
$$

Defina a função

$$
\begin{aligned}
t(s)= & -\frac{1}{2} \zeta\left(s, \Delta^{(1)}\right)+\zeta\left(s, \Delta^{(2)}\right)-\frac{3}{2} \zeta\left(s, \Delta^{(3)}\right)+2 \zeta\left(s, \Delta^{(4)}\right) \\
= & \frac{1}{2} \sum_{k=1}^{\infty} \frac{j_{1, k}^{-2 s}}{l^{-2 s}}-\frac{1}{2} \sum_{k=1}^{\infty} \frac{j_{2, k}^{-2 s}}{l^{-2 s}}+\sum_{n, k=1}^{\infty} n(n+2) \frac{\left(j_{\mu_{1, n}, k}^{\prime}\right)^{-2 s}}{l^{-2 s}}-\sum_{n, k=1}^{\infty} n(n+2) \frac{j_{\mu_{1, n}, k}^{-2 s}}{l^{-2 s}} \\
& +\sum_{n, k=1}^{\infty}(n+1)^{2} \frac{j_{\mu_{0, n}, k}^{-2 s}}{l^{-2 s}}-\frac{1}{2} \sum_{n, k=1}^{\infty}(n+1)^{2} \frac{\tilde{j}_{\mu_{0, n}, k,-}^{-2 s}}{l^{-2 s}}-\frac{1}{2} \sum_{n, k=1}^{\infty}(n+1)^{2} \frac{\tilde{j}_{\mu_{0, n}, k,+}^{-2 s}}{l^{-2 s}} \\
= & l^{2 s}\left(\frac{1}{2} z_{1}(s)-\frac{1}{2} z_{2}(s)+\hat{Z}(s)-Z(s)+Z_{0}(s)-\frac{1}{2} Z_{+}(s)-\frac{1}{2} Z_{-}(s)\right),
\end{aligned}
$$

então

$$
\begin{aligned}
\log T\left(C_{\alpha} S_{l a}^{3}\right) & =t^{\prime}(0) \\
& =\frac{1}{2} z_{1}^{\prime}(0)-\frac{1}{2} z_{2}^{\prime}(0)+\hat{Z}^{\prime}(0)-Z^{\prime}(0)+Z_{0}^{\prime}(0)-\frac{1}{2} Z_{+}^{\prime}(0)-\frac{1}{2} Z_{-}^{\prime}(0) \\
& +\log l^{2}\left(\frac{1}{2} z_{1}(0)-\frac{1}{2} z_{2}(0)+\hat{Z}(0)-Z(0)+Z_{0}(0)-\frac{1}{2} Z_{+}(0)-\frac{1}{2} Z_{-}(0)\right) .
\end{aligned}
$$

Usando as equações (1.22) da seção 1.5, calculamos $z_{1 / 2}(0)$ e $z_{1 / 2}^{\prime}(0)$. Obtemos

$$
\begin{aligned}
\log T\left(C_{\alpha} S_{l a}^{3}\right) & =\left(\frac{1}{4}+\hat{Z}(0)-Z(0)+Z_{0}(0)-\frac{1}{2} Z_{+}(0)-\frac{1}{2} Z_{-}(0)\right) \log l^{2} \\
& +\left(-\log 2+\hat{Z}^{\prime}(0)-Z^{\prime}(0)+Z_{0}^{\prime}(0)-\frac{1}{2} Z_{+}^{\prime}(0)-\frac{1}{2} Z_{-}^{\prime}(0)\right) .
\end{aligned}
$$

Para determinar a parte remanescente, usaremos o corolário 1 do teorema 5. Consideraremos separadamente duas funções $Z(s)-\hat{Z}(s)$ e $2 Z_{0}(s)-Z_{+}(s)-Z_{-}(s)$. No primeiro caso, as seqüências relevantes são as seqüências duplas $S=\left\{n(n+2): j_{\mu_{1, n}, k}^{2}\right\}$ e $\hat{S}=\left\{n(n+2):\left(j_{\mu_{1, n}, k}^{\prime}\right)^{2}\right\}$ e a seqüência simples $U_{1}=\left\{n(n+2): \mu_{1, n}\right\}_{n=1}^{\infty}$, onde $Z(s)=\zeta(s, S)$ e $\hat{Z}(s)=\zeta(s, \hat{S})$. No segundo caso, as seqüências relevantes são as seqüências duplas $S_{0}=\left\{(n+1)^{2}: j_{\mu_{0, n}, k}^{2}\right\}$ e $S_{ \pm}=\left\{(n+1)^{2}:\left(\tilde{j}_{\mu_{0, n, \pm}, k}\right)^{2}\right\}$ e a seqüência simples $U_{0}=\left\{(n+1)^{2}: \mu_{0, n}\right\}_{n=1}^{\infty}$, onde $Z_{0}(s)=\zeta\left(s, S_{0}\right)$ e $Z_{ \pm}(s)=\zeta\left(s, S_{ \pm}\right)$. 
Iniciamos analisando as duas seqüências simples $U_{j}, j=0,1$. Lembremos do lema 9 que

$$
\mu_{0, n}=\sqrt{\nu^{2} n(n+2)+1}, \quad \mu_{1, n}=\nu(n+1) .
$$

Considere primeiro $U_{1}=\left\{n(n+2): \mu_{1, n}\right\}_{n=1}^{\infty}$. Pela definição de $\mu_{1, n}$, é fácil ver que

$$
\zeta\left(s, U_{1}\right)=\nu^{-s}\left(\zeta_{R}(s-2)-\zeta_{R}(s)\right),
$$

e portanto $U_{1}$ é uma seqüência totalmente regular do tipo espectral com ordem infinita, e $\left(U_{1}\right)=\mathrm{g}\left(U_{1}\right)=3$ e $\zeta\left(s, U_{1}\right)$ possui polos simples em $s=1$ e $s=3$ com resíduos:

$$
\begin{aligned}
& \operatorname{Res}_{s=1} \zeta\left(s, U_{1}\right)=\frac{1}{\nu}\left(\log \nu-\gamma-\frac{1}{12}\right), \quad \operatorname{Res}_{s=1} \zeta\left(s, U_{1}\right)=-\frac{1}{\nu}, \\
& \operatorname{Res}_{s=3} \zeta\left(s, U_{1}\right)=\frac{1}{\nu^{3}}(\gamma-\log \nu-\zeta(3)), \quad \operatorname{Res}_{s=3} \zeta\left(s, U_{1}\right)=\frac{1}{\nu^{3}} .
\end{aligned}
$$

Para a seqüência $U_{0}$, temos que $U_{0}=\left\{(n+1)^{2}: \mu_{0, n}\right\}_{n=1}^{\infty}$. Para um número positivo $q$, considere a seqüência

$$
L_{q}=\left\{(n+1)^{2}: \sqrt{n(n+2)+q}\right\}_{n=1}^{\infty} .
$$

Então é claro que

$$
\zeta\left(s, U_{0}\right)=\nu^{-s} \zeta\left(s, L_{\frac{1}{\nu^{2}}}\right) .
$$

A seqüência $L_{0}$ é a seqüência da raiz quadrada dos autovalores positivos do operador Laplaciano sobre a 3 -esfera $S^{3}$ de raio 1 (ver [40]). Então

$$
\zeta\left(2 s, L_{0}\right)=\zeta\left(s, \mathrm{Sp}_{+} \Delta_{S^{3}}\right)
$$

A função zeta $\zeta\left(s, \mathrm{Sp}_{+} \Delta_{S^{3}}\right)$ foi estudada por vários autores. Iremos nos basear em [40]. Usando os resultados em [40], segue que e $\left(\operatorname{Sp}_{+} \Delta_{S^{3}}^{(0)}\right)=\frac{3}{2}, \mathrm{~g}\left(\operatorname{Sp}_{+} \Delta_{S^{3}}^{(0)}\right)=1$, e que $\operatorname{Sp}_{+} \Delta_{S^{3}}^{(0)}$ é uma seqüência do tipo espectral totalmente regular com ordem infinita. Como transladar a seqüência não altera o expoente de convergência e nem o genus (ver [43]), segue que e $\left(U_{0}\right)=$ $\mathrm{g}\left(U_{0}\right)=3$ e que $U_{0}$ é uma seqüência totalmente regular do tipo espectral com ordem infinita. Em [40], está provado ainda que $\zeta\left(s, \mathrm{Sp}_{+} \Delta_{S^{3}}\right)$ possui pólos simples em $s=\frac{3}{2}, \frac{1}{2},-\frac{2 j-1}{2}$, para todo $j>0$, e fórmulas para determinar os resíduos são dadas. Em particular:

$$
\operatorname{Res}_{\substack{1 \\ s=\frac{3}{2}}} \zeta\left(s, \operatorname{Sp}_{+} \Delta_{S^{3}}^{(0)}\right)=\frac{1}{2}, \quad \operatorname{Res}_{s=\frac{1}{2}} \zeta\left(s, \operatorname{Sp}_{+} \Delta_{S^{3}}^{(0)}\right)=\frac{1}{4} .
$$

Assim, $\zeta\left(s, L_{0}\right)$, possui pólos simples em $s=1$ e $s=3$ com os resíduos:

$$
\operatorname{Res}_{s=3} \zeta\left(s, L_{0}\right)=1, \quad \operatorname{Res}_{s=1} \zeta\left(s, L_{0}\right)=\frac{1}{2} .
$$

Expandindo a potência do binômio em $\zeta\left(s, L_{q}\right)$ segue-se que

$$
\zeta\left(s, L_{q}\right)=\zeta\left(s, L_{0}\right)-\frac{s}{2} \zeta\left(s+2, L_{0}\right) q+\sum_{j=2}^{\infty}\left(\begin{array}{c}
-\frac{s}{2} \\
j
\end{array}\right) \zeta\left(s+2 j, L_{0}\right) q^{j},
$$


e portanto

$$
\operatorname{Res}_{s=1} \zeta\left(s, L_{q}\right)=\frac{1}{2}(1-q), \quad \operatorname{Res}_{s=3} \zeta\left(s, L_{q}\right)=1 .
$$

Desta forma temos as expansões

$$
\begin{array}{ll}
\zeta\left(s, U_{0}\right)=\nu^{-s} \zeta\left(s, L_{q}\right)=\frac{1}{2 \nu}\left(1-\frac{1}{\nu^{2}}\right) \frac{1}{s-1}+K_{1}(s), & \text { em } s=1, \\
\zeta\left(s, U_{0}\right)=\nu^{-s} \zeta\left(s, L_{q}\right)=\frac{1}{\nu^{3}} \frac{1}{s-3}+K_{3}(s), & \text { em } s=3,
\end{array}
$$

onde os $K_{j}(s)$ são funções regulares. O próximo passo é analisar as seqüências duplas. O faremos em duas partes:

\section{Parte I}

Nesta primeira parte consideraremos $\hat{Z}(s)-Z(s)$. Logo, consideramos as seqüências $S$ e $\hat{S}$. Usando estimativas para os zeros das funções de Bessel [48] temos que e $(S)=\mathrm{e}(\hat{S})=2$, e os genus relativos são $(2,1,0)$ para as duas seqüências. O fato de $S_{n}$ e $\hat{S}_{n}$ são seqüências totalmente regulares do tipo espectral com ordem infinita, é conseqüência da análise a seguir. Note que usando as representações em produtos (4.26) obtemos as representações a seguir para as funções Gamma associadas as seqüências $S_{n}$ e $\hat{S}_{n}$. Apresentaremos as representações das funções Gamma associadas as seqüências $S_{n} / \mu_{1, n}^{2}$, e $\hat{S}_{n} / \mu_{1, n}^{2}$, que será a que iremos utilizar. Por definição na equação (1.17), com $z=\sqrt{-\lambda}$, temos

$$
\begin{aligned}
\log \Gamma\left(-\lambda, S_{n} /\left(\mu_{1, n}\right)^{2}\right)= & -\log \prod_{k=1}^{\infty}\left(1+\frac{(-\lambda)\left(\mu_{1, n}\right)^{2}}{j_{\mu_{1, n}, k}^{2}}\right) \\
= & -\log I_{\mu_{1, n}}\left(\mu_{1, n} \sqrt{-\lambda}\right)+\left(\mu_{1, n}\right) \log \sqrt{-\lambda} \\
& +\mu_{1, n} \log \left(\mu_{1, n}\right)-\mu_{1, n} \log 2-\log \Gamma\left(\mu_{1, n}+1\right), \\
\log \Gamma\left(-\lambda, \hat{S}_{n} /\left(\mu_{1, n}\right)^{2}\right)= & -\log \prod_{k=1}^{\infty}\left(1+\frac{(-\lambda)\left(\mu_{1, n}\right)^{2}}{\left(j_{\mu_{1, n}, k}^{\prime}\right)^{2}}\right) \\
= & -\log I_{\mu_{1, n}}^{\prime}\left(\mu_{1, n} \sqrt{-\lambda}\right)+\left(\mu_{1, n}-1\right) \log \sqrt{-\lambda} \\
& +\mu_{1, n} \log \left(\mu_{1, n}\right)-\mu_{1, n} \log 2-\log \Gamma\left(\mu_{1, n}+1\right) .
\end{aligned}
$$

Uma primeira conseqüência destas representações é a existência de uma expansão assintótica completa para as funções Gamma $\log \Gamma\left(-\lambda, S_{n}\right)$ e $\log \Gamma\left(-\lambda, \hat{S}_{n}\right)$, $\operatorname{logo} S_{n}$ e $\hat{S}_{n}$ são seqüências do tipo espectral e mais, ambas são seqüências totalmente regulares com ordem infinita.

O Próximo passo consiste em provar que $S$ e $\hat{S}$ são espectralmente decompostas sobre $U_{1}$ com potência $\kappa=2$ e comprimento $\ell=4$, como na definição 13. Temos que mostrar que as funções $\log \Gamma\left(-\lambda, S_{n} / \mu_{1, n}^{2}\right)$ e $\log \Gamma\left(-\lambda, \hat{S}_{n} / \mu_{1, n}^{2}\right)$ possuem as expansões uniformes apropriadas 
para $n$ grande. Como antes, isto segue usando as expansões para as funções de Bessel,

$$
I_{\nu}(\nu z)=\frac{\mathrm{e}^{\nu \sqrt{1+z^{2}}} \mathrm{e}^{\nu \log \frac{z}{1+\sqrt{1+z^{2}}}}}{\sqrt{2 \pi \nu}\left(1+z^{2}\right)^{\frac{1}{4}}}\left(1+U_{1}(z) \frac{1}{\nu}+U_{2}(z) \frac{1}{\nu^{2}}+U_{3}(z) \frac{1}{\nu^{3}}+O\left(\frac{1}{\nu^{4}}\right)\right),
$$

onde

$$
\begin{aligned}
U_{1}(z) & =\frac{1}{8 \sqrt{1+z^{2}}}-\frac{5}{24\left(1+z^{2}\right)^{\frac{3}{2}}} \\
U_{2}(z) & =\frac{9}{128\left(1+z^{2}\right)}-\frac{77}{192\left(1+z^{2}\right)^{2}}+\frac{385}{1152\left(1+z^{2}\right)^{3}}, \\
U_{3}(z) & =\frac{75}{1024\left(1+z^{2}\right)^{\frac{3}{2}}}-\frac{4563}{5120\left(1+z^{2}\right)^{\frac{5}{2}}}+\frac{17017}{9216\left(1+z^{2}\right)^{\frac{7}{2}}}-\frac{85085}{82944\left(1+z^{2}\right)^{\frac{9}{2}}},
\end{aligned}
$$

$\mathrm{e}$

$$
\begin{gathered}
I_{\nu}^{\prime}(\nu z)=\frac{\left(1+z^{2}\right)^{\frac{1}{4}} \mathrm{e}^{\nu \sqrt{1+z^{2}}} \mathrm{e}^{\nu \log \frac{z}{1+\sqrt{1+z^{2}}}}}{\sqrt{2 \pi \nu} z}\left(1+V_{1}(z) \frac{1}{\nu}+V_{2}(z) \frac{1}{\nu^{2}}+V_{3}(z) \frac{1}{\nu^{3}}+O\left(\frac{1}{\nu^{4}}\right)\right), \\
V_{1}(z)=-\frac{3}{8 \sqrt{1+z^{2}}}+\frac{7}{24\left(1+z^{2}\right)^{\frac{3}{2}}}, \\
V_{2}(z)=-\frac{15}{128\left(1+z^{2}\right)}+\frac{33}{64\left(1+z^{2}\right)^{2}}-\frac{455}{1152\left(1+z^{2}\right)^{3}}, \\
V_{3}(z)=-\frac{105}{1024\left(1+z^{2}\right)^{\frac{3}{2}}}+\frac{5577}{5120\left(1+z^{2}\right)^{\frac{5}{2}}}-\frac{6545}{3072\left(1+z^{2}\right)^{\frac{7}{2}}}+\frac{95095}{82944\left(1+z^{2}\right)^{\frac{9}{2}}} .
\end{gathered}
$$

Usando a expansão para o logaritmo da função Gamma de Euler, obtemos, para $n$ grande, uniformemente em $\lambda$, a expansão de $\log \Gamma\left(-\lambda, \hat{S}_{n} / \mu_{1, n}^{2}\right)$ e de $\log \Gamma\left(-\lambda, S_{n} / \mu_{1, n}^{2}\right)$. Fazendo diferença entre estas expansões

$$
\begin{aligned}
\log \Gamma\left(-\lambda, \hat{S}_{n} / \mu_{1, n}^{2}\right) & -\log \Gamma\left(-\lambda, S_{n} / \mu_{1, n}^{2}\right)=\sum_{h=0}^{\infty}\left(\hat{\phi}_{h-1}(\lambda)-\phi_{h-1}(\lambda)\right) \mu_{1, n}^{1-h} \\
& =-\frac{1}{2} \log (1-\lambda)+\left(\hat{\phi}_{1}(\lambda)-\phi_{1}(\lambda)\right) \frac{1}{\mu_{1, n}} \\
& +\left(\hat{\phi}_{2}(\lambda)-\phi_{2}(\lambda)\right) \frac{1}{\mu_{1, n}^{2}}+\left(\hat{\phi}_{3}(\lambda)-\phi_{3}(\lambda)\right) \frac{1}{\mu_{1, n}^{3}}+O\left(\frac{1}{\mu_{1, n}^{4}}\right)
\end{aligned}
$$

com

$$
\begin{aligned}
& \hat{\phi}_{1}(\lambda)-\phi_{1}(\lambda)=\frac{1}{2} \frac{1}{(1-\lambda)^{\frac{1}{2}}}-\frac{1}{2} \frac{1}{(1-\lambda)^{\frac{3}{2}}}, \\
& \hat{\phi}_{2}(\lambda)-\phi_{2}(\lambda)=\frac{1}{4} \frac{1}{(1-\lambda)}-\frac{1}{(1-\lambda)^{2}}+\frac{3}{4} \frac{1}{(1-\lambda)^{3}}, \\
& \hat{\phi}_{3}(\lambda)-\phi_{3}(\lambda)=\frac{11}{48} \frac{1}{(1-\lambda)^{\frac{3}{2}}}-\frac{35}{16} \frac{1}{(1-\lambda)^{\frac{5}{2}}}+\frac{67}{16} \frac{1}{(1-\lambda)^{\frac{7}{2}}}-\frac{107}{48} \frac{1}{(1-\lambda)^{\frac{9}{2}}} .
\end{aligned}
$$

Como e $\left(U_{1}\right)=3$, o maior inteiro $h$ tal que $h-1=\sigma_{h} \leq 3$ é 4 logo $\ell=4$. No entanto, pelo teorema 4, apenas os termos com $\sigma_{h}=1$ e $\sigma_{h}=3$ (ou seja, $h=2,4$ ) aparecem na fórmula do 
teorema 5, como os polos de $\zeta\left(s, U_{1}\right)$ são em $s=1$ e $s=3$. Agora aplicaremos as fórmulas do teorema 5 .

Primeiro pela definição na equação (1.19),

$$
\begin{aligned}
\hat{\Phi}_{1}(s)-\Phi_{1}(s)= & \int_{0}^{\infty} t^{s-1} \frac{1}{2 \pi \mathrm{i}} \int_{\Lambda_{\theta, c}} \frac{\mathrm{e}^{-\lambda t}}{-\lambda}\left(\frac{1}{2} \frac{1}{(1-\lambda)^{\frac{1}{2}}}-\frac{1}{2} \frac{1}{(1-\lambda)^{\frac{3}{2}}}\right) d \lambda d t, \\
\hat{\Phi}_{2}(s)-\Phi_{2}(s)= & \int_{0}^{\infty} t^{s-1} \frac{1}{2 \pi \mathrm{i}} \int_{\Lambda_{\theta, c}} \frac{\mathrm{e}^{-\lambda t}}{-\lambda}\left(\frac{1}{4} \frac{1}{(1-\lambda)}-\frac{1}{(1-\lambda)^{2}}+\frac{3}{4} \frac{1}{(1-\lambda)^{3}}\right) d \lambda d t, \\
\hat{\Phi}_{3}(s)-\Phi_{3}(s)= & \int_{0}^{\infty} t^{s-1} \frac{1}{2 \pi \mathrm{i}} \int_{\Lambda_{\theta, c}} \frac{\mathrm{e}^{-\lambda t}}{-\lambda}\left(\frac{11}{48} \frac{1}{(1-\lambda)^{\frac{3}{2}}}-\frac{35}{16} \frac{1}{(1-\lambda)^{\frac{5}{2}}}\right) d \lambda d t \\
& +\int_{0}^{\infty} t^{s-1} \frac{1}{2 \pi \mathrm{i}} \int_{\Lambda_{\theta, c}} \frac{\mathrm{e}^{-\lambda t}}{-\lambda}\left(\frac{67}{16} \frac{1}{(1-\lambda)^{\frac{7}{2}}}-\frac{107}{48} \frac{1}{(1-\lambda)^{\frac{9}{2}}}\right) d \lambda d t .
\end{aligned}
$$

Calculando estas integrais, usando a fórmula (1.23), obtemos

$$
\begin{aligned}
& \operatorname{Res}_{s=0}\left(\hat{\Phi}_{1}(s)-\Phi_{1}(s)\right)=-1, \quad \operatorname{Res}_{s=0}\left(\hat{\Phi}_{1}(s)-\Phi_{1}(s)\right)=0, \\
& \operatorname{Res}_{s=0}\left(\hat{\Phi}_{2}(s)-\Phi_{2}(s)\right)=\frac{1}{8}, \quad \operatorname{Res}_{s=0}\left(\hat{\Phi}_{2}(s)-\Phi_{2}(s)\right)=0 \text {, } \\
& \operatorname{Res}_{s=0}\left(\hat{\Phi}_{3}(s)-\Phi_{3}(s)\right)=-\frac{2}{315}, \quad \operatorname{Res}_{s=0}\left(\hat{\Phi}_{3}(s)-\Phi_{3}(s)\right)=0 .
\end{aligned}
$$

Com estes resultados e os resíduos da função zeta de $\zeta\left(s, U_{1}\right)$, dados na equação (5.7), segue que

$$
\begin{aligned}
\hat{Z}(0)-Z(0)= & -\hat{A}_{0,1}(0)+A_{0,1}(0)+\frac{1}{2} \operatorname{Res}_{s=1} \zeta\left(s, U_{1}\right) \operatorname{Res}_{s=0}\left(\hat{\Phi}_{1}(s)-\Phi_{1}(s)\right) \\
& +\frac{1}{2} \operatorname{Res}_{s=3} \zeta\left(s, U_{1}\right) \operatorname{Res}_{s=0}\left(\hat{\Phi}_{3}(s)-\Phi_{3}(s)\right) \\
= & -\hat{A}_{0,1}(0)+A_{0,1}(0)
\end{aligned}
$$

$\mathrm{e}$

$$
\begin{aligned}
\hat{Z}^{\prime}(0)-Z^{\prime}(0)= & -\hat{A}_{0,0}(0)-\hat{A}_{0,1}^{\prime}(0)+A_{0,0}(0)+A_{0,1}^{\prime}(0) \\
& +\frac{1}{2} \operatorname{Res}_{s=1} \zeta\left(s, U_{1}\right) \operatorname{Res}_{s=0}\left(\hat{\Phi}_{1}(s)-\Phi_{1}(s)\right) \\
& +\frac{1}{2} \operatorname{Res}_{s=3} \zeta\left(s, U_{1}\right) \operatorname{Res}_{s=0}\left(\hat{\Phi}_{3}(s)-\Phi_{3}(s)\right), \\
= & -\hat{A}_{0,0}(0)+A_{0,0}(0)-\hat{A}_{0,1}^{\prime}(0)+A_{0,1}^{\prime}(0)+\frac{1}{2 \nu}-\frac{1}{315 \nu^{3}} .
\end{aligned}
$$

Para terminar esta parte, por meio da equação (1.21) e o teorema 4, os termos $A_{0,0}(0)$ e $A_{0,1}^{\prime}(0)$, são

$$
\begin{aligned}
& A_{0,0}(s)=\sum_{n=1}^{\infty}\left(a_{0,0, n}-b_{1,0,0} u_{n}^{-1}-b_{3,0,0} u_{n}^{-3}\right) u_{n}^{-2 s} \\
& A_{0,1}(s)=\sum_{n=1}^{\infty}\left(a_{0,1, n}-b_{1,0,1} u_{n}^{-1}-b_{3,0,1} u_{n}^{-3}\right) u_{n}^{-2 s}
\end{aligned}
$$


Logo, precisamos da expansão para $\lambda$ grande das funções $\log \Gamma\left(-\lambda, \hat{S}_{n} / \mu_{1, n}^{2}\right), \hat{\phi}_{1}(\lambda), \hat{\phi}_{3}(\lambda)$, $\log \Gamma\left(-\lambda, S_{n} / \mu_{1, n}^{2}\right), \phi_{1}(\lambda)$ e $\phi_{3}(\lambda)$. Usando expansões clássicas para as funções de Bessel e sua derivada, dadas na equação (4.29), e as fórmulas na equação (1.20), obtemos

$$
\begin{aligned}
& a_{0,0, n}=\frac{1}{2} \log 2 \pi+\left(\mu_{1, n}+\frac{1}{2}\right) \log \mu_{1, n}-\mu_{1, n} \log 2-\log \Gamma\left(\mu_{1, n}+1\right), \\
& a_{0,1, n}=\frac{1}{2}\left(\mu_{1, n}+\frac{1}{2}\right) \text {, } \\
& b_{1,0,0}=-\frac{1}{12}, \quad b_{3,0,0}=\frac{1}{360}, \quad b_{1,0,1}=b_{3,0,1}=0,
\end{aligned}
$$

e

$$
\begin{array}{ll}
\hat{a}_{0,0, n}=\frac{1}{2} \log 2 \pi+\left(\mu_{1, n}+\frac{1}{2}\right) \log \mu_{1, n}-\mu_{1, n} \log 2-\log \Gamma\left(\mu_{1, n}+1\right), \\
\hat{a}_{0,1, n}=\frac{1}{2}\left(\mu_{1, n}-\frac{1}{2}\right), \\
\hat{b}_{1,0,0}=-\frac{1}{12}, \quad \hat{b}_{3,0,0}=\frac{1}{360}, \quad \hat{b}_{1,0,1}=\hat{b}_{3,0,1}=0 .
\end{array}
$$

O que mostra que $A_{0,0}(0)$ e $\hat{A}_{0,0}(0)$ são iguais. Ainda,

$$
\hat{A}_{0,1}(s)-A_{0,1}(s)=-\frac{1}{2} \sum_{n=1}^{\infty} n(n+2) \mu_{1, n}^{-2 s}=-\frac{1}{2} \zeta\left(2 s, U_{1}\right) .
$$

Então

$$
\begin{aligned}
& \hat{A}_{0,1}(0)-A_{0,1}(0)=-\frac{1}{4} \\
& \hat{A}_{0,1}^{\prime}(0)-A_{0,1}^{\prime}(0)=\frac{1}{2} \log \nu-\zeta^{\prime}(-2)-\frac{1}{2} \log 2 \pi
\end{aligned}
$$

Substituindo em (5.9) e (5.10), temos

$$
\begin{aligned}
\hat{Z}(0)-Z(0) & =\frac{1}{4} \\
\hat{Z}^{\prime}(0)-Z^{\prime}(0) & =-\frac{1}{2} \log \nu+\zeta^{\prime}(-2)+\frac{1}{2} \log 2 \pi+\frac{1}{2 \nu}-\frac{1}{315 \nu^{3}} .
\end{aligned}
$$

\section{Parte II}

Trataremos agora de $2 Z_{0}(s)-Z_{+}(s)-Z_{-}(s)$. Então, consideramos as seqüências $S_{0}$ e $S_{ \pm}$. A seqüência $S_{0}$ é análoga a seqüência $S$ analisada na parte anterior. Temos

$$
\begin{aligned}
\log \Gamma\left(-\lambda, S_{0, n} / \mu_{0, n}^{2}\right)= & -\log I_{\mu_{0, n}}\left(\mu_{0, n} \sqrt{-\lambda}\right)+\mu_{0, n} \log \sqrt{-\lambda}+\mu_{0, n} \log \mu_{0, n} \\
& -\mu_{0, n} \log 2-\log \Gamma\left(\mu_{0, n}\right)-\log \mu_{0, n}
\end{aligned}
$$


Usando a expansão uniforme de $\log I_{\mu_{0, n}}\left(\mu_{0, n} \sqrt{-\lambda}\right)$, obtemos a expansão uniforme para $n$ grande de $\log \Gamma\left(-\lambda, S_{0, n} / \mu_{0, n}^{2}\right)$, como segue

$$
\begin{aligned}
\log \Gamma & \left(-\lambda, S_{0, n} / \mu_{0, n}^{2}\right)=\sum_{h=0}^{\infty} \phi_{h-1,0}(\lambda) \mu_{0, n}^{1-h} \\
& =(-\sqrt{1-\lambda}+\log (1+\sqrt{1-\lambda})-\log 2+1-\log \sqrt{-\lambda}) \mu_{0, n} \\
& +\frac{1}{4} \log (1-\lambda)+\left(-U_{1}(\sqrt{-\lambda})-\frac{1}{12}\right) \frac{1}{\mu_{0, n}}+\left(-U_{2}(\sqrt{-\lambda})+\frac{1}{2} U_{1}(\sqrt{-\lambda})^{2}\right) \frac{1}{\mu_{0, n}^{2}} \\
& +\left(-U_{3}(\sqrt{-\lambda})+U_{1}(\sqrt{-\lambda}) U_{2}(\sqrt{-\lambda})-\frac{1}{3} U_{1}(\sqrt{-\lambda})^{3}+\frac{1}{360}\right) \frac{1}{\mu_{0, n}^{3}}+O\left(\frac{1}{\mu_{1, n}^{4}}\right),
\end{aligned}
$$

e assim

$$
\begin{aligned}
& \phi_{1,0}(\lambda)=-\frac{1}{8} \frac{1}{(1-\lambda)^{\frac{1}{2}}}+\frac{5}{24} \frac{1}{(1-\lambda)^{\frac{3}{2}}}-\frac{1}{12} \\
& \phi_{2,0}(\lambda)=-\frac{1}{16} \frac{1}{(1-\lambda)}+\frac{3}{8} \frac{1}{(1-\lambda)^{2}}-\frac{5}{16} \frac{1}{(1-\lambda)^{3}}, \\
& \phi_{3,0}(\lambda)=-\frac{25}{384} \frac{1}{(1-\lambda)^{\frac{3}{2}}}+\frac{531}{640} \frac{1}{(1-\lambda)^{\frac{5}{2}}}-\frac{221}{128} \frac{1}{(1-\lambda)^{\frac{7}{2}}}+\frac{1105}{1152} \frac{1}{(1-\lambda)^{\frac{9}{2}}}+\frac{1}{360} .
\end{aligned}
$$

Considerando as expansões de $\log I_{\mu_{0, n}}\left(\mu_{0, n} \sqrt{-\lambda}\right)$ e de $\phi_{j, 0}(\lambda)$ para $\lambda$ suficientemente grande e, ainda, as definições na equação (1.20), calculamos

$$
\begin{array}{ll}
a_{0,0, n, 0}=\frac{1}{2} \log 2 \pi+\left(\mu_{0, n}+\frac{1}{2}\right) \log \mu_{0, n}-\mu_{0, n} \log 2-\log \Gamma\left(\mu_{0, n}+1\right), \\
a_{0,1, n, 0}=\frac{1}{2}\left(\mu_{0, n}+\frac{1}{2}\right), \\
b_{1,0,0,0}=-\frac{1}{12}, \quad b_{3,0,0,0}=\frac{1}{360}, \quad b_{1,0,1,0}=b_{3,0,1,0}=0 .
\end{array}
$$

A análise para $S_{ \pm}$requer um pouco mais de trabalho; ela se dá com argumentos semelhantes a do teorema 16. Defina as funções

$$
T_{\nu}^{ \pm}(z)= \pm J_{\nu}(z)+z J_{\nu}^{\prime}(z)
$$

Lembrando a definição da função de Bessel em série, que está na equação (4.33), obtemos próximo a $z=0$,

$$
T_{\nu}^{ \pm}(z)=\left(1 \pm \frac{1}{\nu}\right) \frac{z^{\nu}}{2^{\nu} \Gamma(\nu)}
$$

Isto significa que a função $\hat{T}_{\nu}^{ \pm}(z)=z^{-\nu} T_{\nu}^{ \pm}(z)$ é uma função par em $z$. Seja $\tilde{j}_{\nu, k, \pm}$ os zeros positivos de $T_{\nu}^{ \pm}(z)$ ordenados de maneira crescente. Pelo teorema de fatorização de Hadamard, temos a expansão em produto

$$
\hat{T}_{\nu}^{ \pm}(z)=\hat{T}_{\nu}^{ \pm}(z) \prod_{k=-\infty}^{+\infty}\left(1-\frac{z}{\tilde{j}_{\nu, k, \pm}}\right),
$$


e portanto

$$
T_{\nu}^{ \pm}(z)=\left(1 \pm \frac{1}{\nu}\right) \frac{z^{\nu}}{2^{\nu} \Gamma(\nu)} \prod_{k=1}^{\infty}\left(1-\frac{z^{2}}{\tilde{j}_{\nu, k, \pm}^{2}}\right) .
$$

Usando a equação (4.34) obtemos

$$
T_{\nu}^{ \pm}(\mathrm{i} z)=\mathrm{e}^{\frac{\pi}{2} \mathrm{i} \nu}\left( \pm I_{\nu}(z)+z I_{\nu}^{\prime}(z)\right) .
$$

Então, definimos (para $\left.-\pi<\arg (z)<\frac{\pi}{2}\right)$

$$
Q_{\nu}^{ \pm}(z)=\mathrm{e}^{-\frac{\pi}{2} \mathrm{i} \nu} T_{\nu}^{ \pm}(\mathrm{i} z)
$$

e assim

$$
Q_{\nu}^{ \pm}(z)= \pm I_{\nu}(z)+z I_{\nu}^{\prime}(z)=\left(1 \pm \frac{1}{\nu}\right) \frac{z^{\nu}}{2^{\nu} \Gamma(\nu)} \prod_{k=1}^{\infty}\left(1+\frac{z^{2}}{\tilde{j}_{\nu, k, \pm}^{2}}\right)
$$

Com estas representações, obtemos as representações a seguir para as funções Gamma associadas as seqüências $S_{ \pm, n}$. Por definição na equação (1.17), com $z=\sqrt{-\lambda}$, temos

$$
\begin{aligned}
\log \Gamma\left(-\lambda, S_{ \pm, n}\right)= & -\log \prod_{k=1}^{\infty}\left(1+\frac{(-\lambda)}{\tilde{j}_{\mu_{0, n}, k, \pm}^{2}}\right) \\
= & -\log Q_{\mu_{0, n}}^{ \pm}(\sqrt{-\lambda})+\mu_{0, n} \log \sqrt{-\lambda} \\
& -\mu_{0, n} \log 2-\log \Gamma\left(\mu_{0, n}\right)+\log \left(1 \pm \frac{1}{\mu_{0, n}}\right) .
\end{aligned}
$$

Assim $S_{+, n}$ e $S_{-, n}$ são seqüências do tipo espectral e, considerando as expansões, vê-se que ambas são seqüências totalmente regulares com ordem infinita.

Nosso próximo passo será provar que $S_{ \pm}$são espectralmente decompostas sobre $U$ com potência $\kappa=2$ e comprimento $\ell=4$, como na definição 13. Mostremos a expansão uniforme apropriada para $n$ grande de $\log \Gamma\left(-\lambda, S_{ \pm, n} / \mu_{0, n}^{2}\right)$. Temos

$$
\begin{aligned}
\log \Gamma\left(-\lambda, S_{ \pm, n} / \mu_{0, n}^{2}\right)= & -\log Q_{\mu_{0, n}}^{ \pm}\left(\mu_{0, n} \sqrt{-\lambda}\right)+\mu_{0, n} \log \sqrt{-\lambda}+\mu_{0, n} \log \mu_{0, n} \\
& -\mu_{0, n} \log 2-\log \Gamma\left(\mu_{0, n}\right)+\log \left(1 \pm \frac{1}{\mu_{0, n}}\right) .
\end{aligned}
$$

Lembrando as expansões que estão nas seções 4.3 .2 e 4.3.3, obtemos

$$
\begin{aligned}
& Q_{\nu}^{ \pm}(\nu z)=\sqrt{\nu}\left(1+z^{2}\right)^{\frac{1}{4}} \frac{\mathrm{e}^{\nu \sqrt{1+z^{2}}} \mathrm{e}^{\nu \log \frac{z}{1+\sqrt{1+z^{2}}}}}{\sqrt{2 \pi}} \\
&\left(1+W_{1, \pm}(z) \frac{1}{\nu}+W_{2, \pm}(z) \frac{1}{\nu^{2}}+W_{3, \pm}(z) \frac{1}{\nu^{3}}+O\left(\nu^{-4}\right)\right),
\end{aligned}
$$

onde $w=\frac{1}{\sqrt{1+z^{2}}}, \mathrm{e}$

$$
W_{1, \pm}(w)=V_{1}(w) \pm w, \quad W_{2, \pm}(w)=V_{2}(w) \pm w U_{1}(w), \quad W_{3, \pm}(w)=V_{3}(w) \pm w U_{2}(w)
$$


Logo

$$
\begin{array}{llrl}
W_{1,+}(w) & =\frac{5}{8} w+\frac{7}{24} w^{3}, & W_{2,+}(w) & =-\frac{1}{128} w^{2}+\frac{59}{192} w^{4}-\frac{455}{1152} w^{6}, \\
W_{3,+}(w) & =-\frac{33}{1024} w^{3}+\frac{10571}{15360} w^{5}-\frac{16555}{9216} w^{7}+\frac{95095}{82944} w^{9}, \\
W_{1,-}(w) & =-\frac{11}{8} w+\frac{7}{24} w^{3}, & W_{2,-}(w) & =-\frac{31}{128} w^{2}+\frac{139}{192} w^{4}-\frac{455}{1152} w^{6}, \\
W_{3,-}(w) & =-\frac{177}{1024} w^{3}+\frac{22891}{15360} w^{5}-\frac{22715}{9216} w^{7}+\frac{95095}{82944} w^{9} .
\end{array}
$$

Isto nos fornece que

$$
\begin{aligned}
& \log \Gamma\left(-\lambda, S_{n, \pm} / \mu_{0, n}^{2}\right)=\sum_{h=0}^{\infty} \phi_{h-1, \pm}(\lambda) \mu_{n}^{1-h} \\
& =(1-\sqrt{1-\lambda}+\log (1+\sqrt{1-\lambda})-\log 2) \mu_{0, n}-\frac{1}{4} \log (1-\lambda) \\
& +\left(-W_{1, \pm}(\sqrt{-\lambda}) \pm 1-\frac{1}{12}\right) \frac{1}{\mu_{0, n}}+\left(-W_{2, \pm}(\sqrt{-\lambda})+\frac{1}{2} W_{1, \pm}^{2}(\sqrt{-\lambda})-\frac{1}{2}\right) \frac{1}{\mu_{0, n}^{2}} \\
& +\left(W_{1, \pm}(\sqrt{-\lambda}) W_{2, \pm}(\sqrt{-\lambda})-W_{3, \pm}(\sqrt{-\lambda})-\frac{1}{3} W_{1, \pm}^{3}(\sqrt{-\lambda}) \pm \frac{1}{3}+\frac{1}{360}\right) \mu_{0, n}^{-3}+O\left(\mu_{0, n}^{-4}\right)
\end{aligned}
$$

e assim

$$
\begin{aligned}
& \phi_{1,+}(\lambda)=-\frac{5}{8} \frac{1}{(1-\lambda)^{\frac{1}{2}}}-\frac{7}{24} \frac{1}{(1-\lambda)^{\frac{3}{2}}}+\frac{11}{12} \\
& \phi_{1,-}(\lambda)=\frac{11}{8} \frac{1}{(1-\lambda)^{\frac{1}{2}}}-\frac{7}{24} \frac{1}{(1-\lambda)^{\frac{3}{2}}}-\frac{13}{12} \\
& \phi_{2,+}(\lambda)=\frac{3}{16} \frac{1}{1-\lambda}-\frac{1}{8} \frac{1}{(1-\lambda)^{2}}+\frac{7}{16} \frac{1}{(1-\lambda)^{3}}-\frac{1}{2} \\
& \phi_{2,-}(\lambda)=\frac{19}{16} \frac{1}{1-\lambda}-\frac{9}{8} \frac{1}{(1-\lambda)^{2}}+\frac{7}{16} \frac{1}{(1-\lambda)^{3}}-\frac{1}{2}, \\
& \phi_{3,+}(\lambda)=-\frac{17}{384} \frac{1}{(1-\lambda)^{\frac{3}{2}}}-\frac{389}{640} \frac{1}{(1-\lambda)^{\frac{5}{2}}}+\frac{203}{128} \frac{1}{(1-\lambda)^{\frac{7}{2}}}-\frac{1463}{1152} \frac{1}{(1-\lambda)^{\frac{9}{2}}}+\frac{121}{360} \\
& \phi_{3,-}(\lambda)=\frac{527}{384} \frac{1}{(1-\lambda)^{\frac{3}{2}}}-\frac{1989}{640} \frac{1}{(1-\lambda)^{\frac{5}{2}}}+\frac{427}{128} \frac{1}{(1-\lambda)^{\frac{7}{2}}}-\frac{1463}{1152} \frac{1}{(1-\lambda)^{\frac{9}{2}}}-\frac{119}{360}
\end{aligned}
$$

Por meio da equação (1.21) e o teorema 4, os termos $A_{0,0}(s)$ e $A_{0,1}(s)$, são

$$
\begin{aligned}
& A_{0,0, \pm}(s)=\sum_{n=1}^{\infty}\left(a_{0,0, n, \pm}-b_{1,0,0, \pm} u_{n}^{-1}-b_{3,0,0, \pm} u_{n}^{-3}\right) u_{n}^{-2 s} \\
& A_{0,1, \pm}(s)=\sum_{n=1}^{\infty}\left(a_{0,1, n, \pm}-b_{1,0,1, \pm} u_{n}^{-1}-b_{3,0,1, \pm} u_{n}^{-3}\right) u_{n}^{-2 s}
\end{aligned}
$$

Assim, precisamos das expansões para $\lambda$ grande das funções $\log \Gamma\left(-\lambda, S_{n, \pm} / \mu_{0, n}^{2}\right), \phi_{1, \pm}(\lambda)$ e $\phi_{3, \pm}(\lambda)$. Usando as equações (4.29), (5.11) e a definição de $Q_{\nu}^{ \pm}(z)$, obtemos que

$$
Q_{\nu}^{ \pm}(z) \sim \frac{\sqrt{z} \mathrm{e}^{z}}{\sqrt{2 \pi}}\left(1+\sum_{k=1}^{\infty} b_{k} z^{-k}\right)+O\left(\mathrm{e}^{-z}\right),
$$


para $z$ grande, onde os $b_{k}$ são números reais. Portanto,

$$
\begin{aligned}
\log \Gamma\left(-\lambda, S_{n, \pm} / \mu_{0, n}^{2}\right)= & -\mu_{0, n} \sqrt{-\lambda}+\frac{1}{2}\left(\mu_{0, n}-\frac{1}{2}\right) \log (-\lambda)+\frac{1}{2} \log 2 \pi \\
& +\left(\mu_{0, n}-\frac{1}{2}\right) \log \mu_{0, n}-\log 2^{\mu_{0, n}} \Gamma\left(\mu_{0, n}\right) \\
& +\log \left(1 \pm \frac{1}{\mu_{0, n}}\right)+O\left(\frac{1}{\sqrt{-\lambda}}\right) .
\end{aligned}
$$

Então,

$$
\begin{aligned}
& a_{0,0, n, \pm}=\frac{1}{2} \log 2 \pi+\left(\mu_{0, n}-\frac{1}{2}\right) \log \mu_{0, n}-\log 2^{\mu_{0, n}} \Gamma\left(\mu_{0, n}\right)+\log \left(1 \pm \frac{1}{\mu_{0, n}}\right), \\
& a_{0,1, n, \pm}=\frac{1}{2}\left(\mu_{0, n}-\frac{1}{2}\right), \\
& b_{1,0,0,+}=-\frac{11}{12}, \quad b_{3,0,0,+}=\frac{121}{360}, \quad b_{1,0,1, \pm}=b_{3,0,1, \pm}=0, \\
& b_{1,0,0,-}=-\frac{13}{12}, \quad b_{3,0,0,+}=\frac{119}{360} .
\end{aligned}
$$

Usando estes coeficiente e os coeficientes que obtemos para a seqüência $S_{0}$, concluímos que

$$
2 A_{0,0,0}(s)-A_{0,0,+}(s)-A_{0,0,-}(s)=-\sum_{n=1}^{\infty} \log \left(1-\frac{1}{\mu_{0, n}^{2}}\right) \frac{(n+1)^{2}}{\mu_{0, n}^{2 s}},
$$

$\mathrm{e}$

$$
2 A_{0,1,0}(s)-A_{0,1,+}(s)-A_{0,1,-}(s)=\sum_{n=1}^{\infty} \frac{(n+1)^{2}}{\mu_{0, n}^{2 s}} .
$$

Agora, utilizamos os resultados obtidos com a expansão uniforme da soma do logaritmos das funções Gamma:

$$
\begin{aligned}
2 \log \Gamma\left(-\lambda, S_{0, n} / \mu_{0, n}^{2}\right) & -\log \Gamma\left(-\lambda, S_{n,+} / \mu_{0, n}^{2}\right)-\log \Gamma\left(-\lambda, S_{n,-} / \mu_{0, n}^{2}\right)=\sum_{h=1}^{\infty} \phi_{h-1}(\lambda) \mu_{0, n}^{1-h} \\
& =\log (1-\lambda)+\sum_{h=2}^{4} \phi_{h-1}(\lambda) \frac{1}{\mu_{0, n}^{h-1}}+O\left(\frac{1}{\mu_{0, n}^{4}}\right) .
\end{aligned}
$$

onde

$$
\phi_{h-1}(\lambda)=2 \phi_{h-1,0}(\lambda)-\phi_{h-1,+}(\lambda)-\phi_{h-1,-}(\lambda)
$$

e

$$
\begin{aligned}
& \phi_{1}(\lambda)=-\frac{1}{(1-\lambda)^{\frac{1}{2}}}+\frac{1}{(1-\lambda)^{\frac{3}{2}}}, \\
& \phi_{2}(\lambda)=-\frac{3}{2} \frac{1}{1-\lambda}+2 \frac{1}{(1-\lambda)^{2}}-\frac{3}{2} \frac{1}{(1-\lambda)^{3}}+1, \\
& \phi_{3}(\lambda)=-\frac{35}{24} \frac{1}{(1-\lambda)^{\frac{3}{2}}}+\frac{43}{8} \frac{1}{(1-\lambda)^{\frac{5}{2}}}-\frac{67}{8} \frac{1}{(1-\lambda)^{\frac{7}{2}}}+\frac{107}{24} \frac{1}{(1-\lambda)^{\frac{9}{2}}} .
\end{aligned}
$$


Seja $\Phi_{h-1}(s)=2 \Phi_{h-1,0}(s)-\Phi_{h-1,+}(s)-\Phi_{h-1,-}(s)$. Usando a definição na equação (1.19) e a fórmula (1.23), temos

$$
\begin{aligned}
& \Phi_{1}(s)=\frac{2 \Gamma\left(s+\frac{1}{2}\right)}{\sqrt{\pi}}, \\
& \Phi_{2}(s)=-\frac{\Gamma(s+1)}{2}\left(5+5 s+\frac{3}{2} s^{2}\right), \\
& \Phi_{3}(s)=\frac{\Gamma\left(s+\frac{3}{2}\right)}{\sqrt{\pi}}\left(\frac{428}{315}+\frac{22}{35} s+\frac{214}{315} s^{2}\right),
\end{aligned}
$$

e assim

$$
\begin{array}{ll}
\operatorname{Res}_{s=0} \Phi_{1}(s)=2, & \operatorname{Res}_{s=0} \Phi_{1}(s)=0, \\
\operatorname{Res}_{s=0} \Phi_{2}(s)=-\frac{5}{2}, & \operatorname{Res}_{s=0} \Phi_{2}(s)=0, \\
\operatorname{Res}_{s=0} \Phi_{3}(s)=\frac{214}{315}, & \operatorname{Res}_{s=0} \Phi_{3}(s)=0 .
\end{array}
$$

Com estes resultados e os resíduos da função $\zeta\left(s, U_{0}\right)$, nas fórmulas dadas no teorema 5 , obtemos

$$
\begin{aligned}
2 Z_{0}(0)-Z_{+}(0)-Z_{-}(0)= & -2 A_{0,1,0}(0)+A_{0,1,+}(0)+A_{0,1,-}(0) \\
2 Z_{0}^{\prime}(0)-Z_{+}^{\prime}(0)-Z_{-}^{\prime}(0)= & -2 A_{0,0,0}(0)+A_{0,0,+}(0)+A_{0,0,-}(0)-2 A_{0,1,0}^{\prime}(0) \\
& +A_{0,1,+}^{\prime}(0)+A_{0,1,-}^{\prime}(0)+\frac{1}{2 \nu}\left(1-\frac{1}{\nu^{2}}\right)+\frac{107}{315 \nu^{3}} .
\end{aligned}
$$

Lembre que

$$
\begin{aligned}
2 A_{0,1,0}(s)-A_{0,1,+}(s)-A_{0,1,-}(s) & =\sum_{n=1}^{\infty} \frac{(n+1)^{2}}{\mu_{0, n}^{2 s}} \\
& =\nu^{-2 s} \zeta\left(2 s, U_{0}\right)=\nu^{-2 s} \zeta\left(s, \operatorname{Sp}_{+} \Delta_{S^{3}}+\frac{1}{\nu^{2}}\right)
\end{aligned}
$$

o que nos dá (ver [40])

$$
2 A_{0,1,0}(0)-A_{0,1,+}(0)-A_{0,1,-}(0)=\zeta\left(0, \mathrm{Sp}_{+} \Delta_{S^{3}}+\frac{1}{\nu^{2}}\right)=-1,
$$

e assim

$$
2 Z_{0}(0)-Z_{+}(0)-Z_{-}(0)=-2 A_{0,1,0}(0)+A_{0,1,+}(0)+A_{0,1,-}(0)=1 .
$$

Para tratar o outro termo, procederemos como segue. Uma vez que

$$
2 A_{0,0,0}(s)-A_{0,0,+}(s)-A_{0,0,-}(s)=-\sum_{n=1}^{\infty}(n+1)^{2} \log \frac{\mu_{0, n}^{2}-1}{\mu_{0, n}^{2}} \mu_{0, n}^{-2 s},
$$


temos

$$
\begin{aligned}
A(s) & =2 A_{0,0,0}(s)-A_{0,0,+}(s)-A_{0,0,-}(s)+2 A_{0,1,0}^{\prime}(s)-A_{0,1,+}^{\prime}(s)-A_{0,1,-}^{\prime}(s) \\
& =-\sum_{n=1}^{\infty}(n+1)^{2} \log \left(\mu_{0, n}^{2}-1\right) \mu_{0, n}^{-2 s} .
\end{aligned}
$$

Lembrando a definição de $\mu_{0, n}$,

$$
\begin{aligned}
A(s)= & -\sum_{n=1}^{\infty}(n+1)^{2} \log \left(\nu^{2} n(n+2)\right) \mu_{0, n}^{-2 s} \\
= & -2 \log \nu \sum_{n=1}^{\infty}(n+1)^{2} \mu_{0, n}^{-2 s}-\sum_{n=1}^{\infty}(n+1)^{2} \log (n(n+2)) \mu_{0, n}^{-2 s} \\
= & -2(\log \nu) \nu^{-2 s} \sum_{j=0}^{\infty}\left(\begin{array}{c}
-s \\
j
\end{array}\right) \zeta\left(s+j, \mathrm{Sp}_{+} \Delta_{S^{3}}\right) \nu^{-2 j} \\
& +\nu^{-2 s} \sum_{j=0}^{\infty}\left(\begin{array}{c}
-s \\
j
\end{array}\right) \zeta^{\prime}\left(s+j, \mathrm{Sp}_{+} \Delta_{S^{3}}\right) \nu^{-2 j},
\end{aligned}
$$

e portanto

$$
\begin{aligned}
A(0) & =-2 \zeta\left(0, \mathrm{Sp}_{+} \Delta_{S^{3}}\right) \log \nu+\zeta^{\prime}\left(0, \mathrm{Sp}_{+} \Delta_{S^{3}}\right) \\
& =2 \log \nu+2 \zeta^{\prime}(-2)+2 \zeta^{\prime}(0)+\log 2 .
\end{aligned}
$$

Isto nos fornece

$$
\begin{aligned}
2 Z_{0}^{\prime}(0)-Z_{+}^{\prime}(0)-Z_{-}^{\prime}(0) & =-A(0)+\frac{1}{2 \nu}\left(1-\frac{1}{\nu^{2}}\right)+\frac{107}{315 \nu^{3}} \\
& =-2 \log \nu-2 \zeta^{\prime}(-2)+\log \pi+\frac{1}{2 \nu}\left(1-\frac{1}{\nu^{2}}\right)+\frac{107}{315 \nu^{3}} .
\end{aligned}
$$

Podemos agora determinar o valor da equação (5.6)

$$
\begin{aligned}
\log T\left(C_{\alpha} S_{l a}^{3}\right)= & \left(\frac{1}{4}+\frac{1}{4}+\frac{1}{2}\right) \log l^{2} \\
& -\log 2-\frac{1}{2} \log \nu+\zeta^{\prime}(-2)+\frac{1}{2} \log 2 \pi+\frac{1}{2 \nu}-\frac{1}{315 \nu^{3}} \\
& -\log \nu-\zeta^{\prime}(-2)+\frac{1}{2} \log \pi+\frac{1}{4 \nu}\left(1-\frac{1}{\nu^{2}}\right)+\frac{107}{630 \nu^{3}} \\
= & \frac{1}{2} \log \frac{\pi^{2} l^{4}}{2 \nu^{3}}+\frac{3}{4} \frac{1}{\nu}-\frac{1}{12 \nu^{3}} .
\end{aligned}
$$

E o resultado segue.

Com os teoremas 16 e 17 temos que,

$$
\begin{aligned}
& \log T\left(D_{l}^{3}\right)=\frac{1}{2} \log \frac{4 \pi l^{3}}{3}+\frac{1}{2} \log 2+\frac{1}{4}, \\
& \log T\left(D_{l}^{4}\right)=\frac{1}{2} \log \frac{\pi^{2} l^{4}}{2}+\frac{2}{3},
\end{aligned}
$$


que são os mesmos resultados obtidos utilizando a fórmula de Brüning e Ma, teorema 10. Agora, utilizando o teorema principal de [13], descrito na seção 4.2, temos

$$
\begin{aligned}
\log T\left(D_{l}^{3}\right) & =\frac{1}{2} \log \frac{4 \pi l^{3}}{3}+\frac{1}{2} \log 2, \\
\log T\left(D_{l}^{4}\right) & =\frac{1}{2} \log \frac{\pi^{2} l^{4}}{2}-\frac{1}{2},
\end{aligned}
$$

o que não confere com os cálculos explícitos feitos nos teoremas 16 e 17. Logo, a fórmula apresentada por Dai e Fang em [13] não está correta.

\subsection{Motivação para a generalização no caso ímpar e a conjec- tura}

Nesta seção apresentamos a motivação para a conjectura 1, cuja o propósito é generalizar o teorema 10 para alguns casos de variedades com singularidades do tipo cônico. E ainda sendo a contribuição do bordo na variedade um fato local, um cálculo (ao menos formal) deste termo pode ser feito.

Lema 10. Considere as duas métrica

$$
\begin{aligned}
& g_{1}=d r \otimes d r+a^{2} r^{2} g_{S^{n}}, \\
& g_{0}=d r \otimes d r+a^{2} l^{2} g_{S^{n}},
\end{aligned}
$$

para $C_{\alpha} S_{l a}^{n}$, onde $a=\operatorname{sen} \alpha$. Então, $(p>0)$

$$
\begin{aligned}
& \log \frac{T_{\text {abs }}\left(C_{\alpha} S_{l a}^{2 p}, g_{1}\right)}{T_{\mathrm{abs}}\left(C_{\alpha} S_{l a}^{2 p}, g_{0}\right)}=\frac{a^{2 p}}{8} \sum_{j=0}^{\left[p-\frac{1}{2}\right]} \frac{1}{j !(p-j) !} \sum_{h=0}^{j}\left(\begin{array}{l}
j \\
h
\end{array}\right) \frac{(-1)^{h}}{(p-j+h) a^{2(j-h)}} \chi\left(S_{l a}^{2 p}\right) \\
& \log \frac{T_{\mathrm{abs}}\left(C_{\alpha} S_{l a}^{2 p-1}, g_{1}\right)}{T_{\mathrm{abs}}\left(C_{\alpha} S_{l a}^{2 p-1}, g_{0}\right)} \\
& =\sum_{j=0}^{p-1} \frac{2^{p-j}}{j !(2(p-j)-1) ! !} \sum_{h=0}^{j}\left(\begin{array}{l}
j \\
h
\end{array}\right) \frac{(-1)^{h}}{(2(p-j+h)-1) a^{2(j-h)}} \frac{a^{2 p-1}(2 p-1) !}{4^{p}(p-1) !} .
\end{aligned}
$$

Demonstração. A prova é uma generalização dos lemas 5 e 6. Usaremos a notação da seção 2.4. Sejam $\omega_{j}=\left(\omega_{j}\right)^{\alpha}{ }_{\beta}$ a 1-forma conexão de $C_{\alpha} S_{l \operatorname{sen} \alpha}^{m-1}$ associada a métrica $g_{j}$, seja $\Theta=\Theta^{\alpha}{ }_{\beta}$ a 2-forma curvatura de $S_{l a}^{m-1}$ com a métrica euclidiana padrão e $R^{T C_{\alpha} S_{l \operatorname{sen} \alpha}^{m-1}}$ (onde denotaremos as entradas da matriz por $R_{\beta}^{\alpha}$ ) a 2-forma curvatura de $C_{\alpha} S_{l \operatorname{sen} \alpha}^{m-1}$ associada a $g_{1}$. Lembremos 
as fórmulas já definidas no capítulo 2, onde $i$ denota a inclusão do bordo (ver equações (2.15)):

$$
\begin{aligned}
& \mathcal{S}_{j}=\frac{1}{2} \sum_{k=1}^{m-1}\left(i^{*} \omega_{j}-i^{*} \omega_{0}\right)^{r}{ }_{\theta_{k}} \hat{e}^{\theta_{k}} \\
& \hat{\Omega}=R^{T C_{\alpha} S_{l \operatorname{sen} \alpha}^{m-1}} \mid S_{l a}^{m-1}=\frac{1}{2} \sum_{k, l=1}^{m-1} i^{*} R^{\theta_{k}}{ }_{\theta_{l}} \hat{e}^{\theta_{k}} \wedge \hat{e}^{\theta_{l}} \\
& \mathcal{R}=\hat{\Theta}=\frac{1}{2} \sum_{k, l=1}^{m-1} \Theta^{\theta_{k}}{ }_{\theta_{l}} \hat{e}^{\theta_{k}} \wedge \hat{e}^{\theta_{l}} .
\end{aligned}
$$

Cálculos diretos, a partir das métricas $g_{j}$, nos permitem obter fórmulas explícitas para estas formas. Os cálculos aqui são muito similares aos feitos para os discos no lema 6, por isso não o repetiremos aqui. Encontramos que as entradas não nulas para as matrizes que aparecem nas equações (5.12) são

$$
\begin{aligned}
& \left(i^{*} \omega_{1}-i^{*} \omega_{0}\right)^{r}{ }_{\theta_{i}}=-a \prod_{j=i+1}^{m-1} \operatorname{sen} \theta_{j} d \theta_{i}=-\frac{1}{l} e^{\theta_{i}}, \\
& i^{*} R^{\theta_{i}}=\left(1-a^{2}\right) \prod_{j=i+1}^{k} \operatorname{sen} \theta_{j} \prod_{s=k+1}^{m-1} \operatorname{sen}^{2} \theta_{s} d \theta_{i} \wedge d \theta_{k}=\left(\frac{1}{l^{2} a^{2}}-\frac{1}{l^{2}}\right) e^{\theta_{i}} \wedge e^{\theta_{k}}, i<k, \\
& \Theta^{\theta_{i}}{ }_{\theta_{k}}=\prod_{j=i+1}^{k} \operatorname{sen} \theta_{j} \prod_{s=k+1}^{m-1} \operatorname{sen}^{2} \theta_{s} d \theta_{i} \wedge d \theta_{k}=\frac{1}{l^{2} a^{2}} e^{\theta_{i}} \wedge e^{\theta_{k}}, \quad i<k .
\end{aligned}
$$

Note que,

$$
\mathcal{S}_{1}^{2}=-\frac{1}{4 l^{2}} \sum_{i, k=1}^{m-1} e^{\theta_{i}} \wedge e^{\theta_{k}} \wedge \hat{e}^{\theta_{i}} \wedge \hat{e}^{\theta_{k}}
$$

É fácil ver que $\mathcal{R}=\hat{\Omega}-2 \mathcal{S}_{1}^{2}$, como na equação (2.18), e ainda que $\mathcal{R}=-\frac{2}{a^{2}} \mathcal{S}_{1}^{2}$. Precisamos apenas determinar $B\left(\nabla_{1}^{T C_{\alpha} S_{l \operatorname{sen} \alpha}^{m-1}}\right)$. A equação (2.16) para $g_{1}$ fica

$$
\begin{aligned}
B\left(\nabla_{1}^{\left.T C_{\alpha} S_{l \operatorname{sen} \alpha}^{m-1}\right)}\right. & =\frac{1}{2} \int_{0}^{1} \int^{B} \mathrm{e}^{\left(\frac{1}{a^{2}}-u^{2}\right) \mathcal{S}_{1}^{2}} \sum_{k=1}^{\infty} \frac{1}{\Gamma\left(\frac{k}{2}+1\right)} u^{k-1} \mathcal{S}_{1}^{k} d u \\
& =\frac{1}{2} \int^{B} \sum_{j=0, k=1}^{\infty} \frac{1}{j ! \Gamma\left(\frac{k}{2}+1\right)} \int_{0}^{1}\left(\frac{1}{a^{2}}-u^{2}\right)^{j} u^{k-1} d u \mathcal{S}_{1}^{k+2 j} \\
& =\frac{1}{2} \int^{B} \sum_{j=0, k=1}^{\infty} \frac{1}{j ! \Gamma\left(\frac{k}{2}+1\right)} \sum_{h=0}^{j}\left(\begin{array}{l}
j \\
h
\end{array}\right) \frac{(-1)^{h}}{(2 h+k) a^{2(j-h)}} \mathcal{S}_{1}^{k+2 j}
\end{aligned}
$$

Como a integral de Berezin é identicamente nula sempre que $k+2 j \neq m-1$, obtemos

$$
\begin{aligned}
B & \left(\nabla_{1}^{T C_{\alpha} S_{l \operatorname{sen} \alpha}^{m-1}}\right) \\
& =\frac{1}{2} \sum_{j=0}^{\left[\frac{m}{2}-1\right]} \frac{1}{j ! \Gamma\left(\frac{m-2 j+1}{2}\right)} \sum_{h=0}^{j}\left(\begin{array}{l}
j \\
h
\end{array}\right) \frac{(-1)^{h}}{(m-2(j-h)-1) a^{2(j-h)}} \int^{B} \mathcal{S}_{1}^{m-1} .
\end{aligned}
$$


Agora, trataremos independentemente os casos $m$ par e $m$ ímpar.

Primeiro, assuma que $m$ seja ímpar, $m=2 p+1(p \geq 0)$. Então, usando a equação (2.14), a equação (5.13) fica

$$
\begin{aligned}
B\left(\nabla_{1}^{\left.T C_{\alpha} S_{l \operatorname{sen} \alpha}^{2 p}\right)}\right. & =\frac{1}{4} \sum_{j=0}^{\left[p-\frac{1}{2}\right]} \frac{1}{j !(p-j) !} \sum_{h=0}^{j}\left(\begin{array}{l}
j \\
h
\end{array}\right) \frac{(-1)^{h}}{(p-j+h) a^{2(j-h)}} \int^{B} \mathcal{S}_{1}^{2 p} \\
& =\frac{1}{4} \sum_{j=0}^{\left[p-\frac{1}{2}\right]} \frac{1}{j !(p-j) !} \sum_{h=0}^{j}\left(\begin{array}{l}
j \\
h
\end{array}\right) \frac{(-1)^{h}}{(p-j+s) a^{2(j-h)}} \int^{B} \frac{\left(-a^{2}\right)^{p}}{2^{p}} \mathcal{R}^{p} \\
& =\frac{a^{2 p}}{4} \sum_{j=0}^{\left[p-\frac{1}{2}\right]} \frac{1}{j !(p-j) !} \sum_{h=0}^{j}\left(\begin{array}{l}
j \\
h
\end{array}\right) \frac{(-1)^{h}}{(p-j+h) a^{2(j-h)}} \int^{B} \mathrm{e}^{-\frac{\mathcal{R}}{2}} \\
& =\frac{a^{2 p}}{4} \sum_{j=0}^{\left[p-\frac{1}{2}\right]} \frac{1}{j !(p-j) !} \sum_{h=0}^{j}\left(\begin{array}{l}
j \\
h
\end{array}\right) \frac{(-1)^{h}}{(p-j+h) a^{2(j-h)}} P f\left(\frac{\Theta}{2 \pi}\right) \\
& =\frac{a^{2 p}}{4} \sum_{j=0}^{\left[p-\frac{1}{2}\right]} \frac{1}{j !(p-j) !} \sum_{h=0}^{j}\left(\begin{array}{l}
j \\
h
\end{array}\right) \frac{(-1)^{h}}{(p-j+h) a^{2(j-h)}} e\left(S_{l a}^{2 p}, g_{E}\right)
\end{aligned}
$$

onde $e\left(S^{2 p}, g_{E}\right)$ é a classe de Euler de $\left(S^{2 p}, g_{E}\right)$. Portanto,

$$
\begin{aligned}
\frac{1}{2} \int_{S_{l \operatorname{sen} \alpha}^{2 p}} B\left(\nabla_{1}^{\left.T C_{\alpha} S_{l \operatorname{sen} \alpha}^{2 p}\right)}\right. \\
=\frac{a^{2 p}}{8} \sum_{j=0}^{\left[p-\frac{1}{2}\right]} \frac{1}{j !(p-j) !} \sum_{h=0}^{j}\left(\begin{array}{l}
j \\
h
\end{array}\right) \frac{(-1)^{h}}{(p-j+h) a^{2(j-h)}} \int_{S_{l a}^{2 p}} e\left(S_{l a}^{2 p}, g_{E}\right) \\
=\frac{a^{2 p}}{8} \sum_{j=0}^{\left[p-\frac{1}{2}\right]} \frac{1}{j !(p-j) !} \sum_{h=0}^{j}\left(\begin{array}{l}
j \\
h
\end{array}\right) \frac{(-1)^{h}}{(p-j+h) a^{2(j-h)}} \chi\left(S_{l a}^{2 p}\right) .
\end{aligned}
$$

Assuma agora que $m$ seja par, $m=2 p(p \geq 1)$. Então, a equação (5.13) fica

$$
\begin{aligned}
& B\left(\nabla_{1} T C_{\alpha} S_{l \operatorname{sen} \alpha}^{2 p-1}\right) \\
& \quad=\frac{1}{2} \sum_{j=0}^{p-1} \frac{1}{j ! \Gamma\left(p-j+\frac{1}{2}\right)} \sum_{h=0}^{j}\left(\begin{array}{l}
j \\
h
\end{array}\right) \frac{(-1)^{h}}{(2(p-j+h)-1) a^{2(j-h)}} \int^{B} \mathcal{S}_{1}^{2 p-1} .
\end{aligned}
$$

Calcularemos $\int^{B} \mathcal{S}_{1}^{2 p-1}$. Lembrando que

$$
\mathcal{R}=-\frac{2}{a^{2}} \mathcal{S}_{1}^{2}
$$

obtemos

$$
\begin{aligned}
\int^{B} \mathcal{S}_{1}^{2 p-1} & =\int^{B} \mathcal{S}_{1} \mathcal{S}_{1}^{2 p-2} \\
& =\frac{(-1)^{p-1} a^{2 p-2}}{2^{p-1}} \int^{B} \mathcal{S}_{1} \mathcal{R}^{p-1}
\end{aligned}
$$


Usando a definição explicita destas formas, dadas na equação (5.12), temos

$$
\begin{aligned}
\int^{B} \mathcal{S}_{1}^{2 p-1} & =\frac{(-1)^{p-1} a^{2 p-2}}{2^{2 p-1}} \int^{B}\left(\sum_{k=1}^{2 p-1}\left(i^{*} \omega_{1}-i^{*} \omega_{0}\right)^{r}{ }_{\theta_{k}} \hat{\mathrm{e}}^{\theta_{k}}\right)\left(\sum_{i, j=1}^{2 p-1} \Theta^{\theta_{i}}{ }_{\theta_{j}} \hat{\mathrm{e}}^{\theta_{i}} \wedge \hat{e}^{\theta_{j}}\right)^{p-1} \\
& =\frac{(-1)^{p} a^{2 p-1}}{2^{2 p-1}} c_{B} \sum_{\substack{\sigma \in S_{2 p} \\
\sigma(1)=1}} \operatorname{sgn}(\sigma)\left(i^{*} \omega_{1}-i^{*} \omega_{0}\right)_{\sigma(2)}^{1}\left(\Omega_{0}\right)_{\sigma(3)}^{\sigma(3)} \ldots\left(\Omega_{0}\right)_{\sigma(2 p-1)}^{\sigma(2 p)},
\end{aligned}
$$

onde $c_{B}=\frac{(-1)^{p(2 p-1)}}{\pi^{\frac{2 p-1}{2}}}$. Usando o mesmo argumento da parte final da prova do lema 6 , obtemos

$$
\int^{B} \mathcal{S}_{1}^{2 p-1}=c_{B} \frac{(-1)^{p} a^{2 p-1}(2 p-1) !}{2^{p-1} 2^{p}} \prod_{j=2}^{2 p-1}\left(\operatorname{sen} \theta_{j}\right)^{j-1} d \theta_{1} \wedge \ldots \wedge d \theta_{2 p-1} .
$$

Então,

$$
\begin{aligned}
\int_{S_{l a}^{2 p-1}} \int^{B} \mathcal{S}_{1}^{2 p-1} & =\frac{(-1)^{p(2 p-1)}}{\pi^{\frac{2 p-1}{2}}} \frac{(-1)^{p} a^{2 p-1}(2 p-1) !}{2^{p-1} 2^{p}(l a)^{2 p-1}} \operatorname{Vol}\left(S_{l a}^{2 p-1}\right) \\
& =\frac{(-1)^{p(2 p-1)}}{\pi^{\frac{2 p-1}{2}}} \frac{(-1)^{p} a^{2 p-1}(2 p-1) !}{2^{p-1} 2^{p}(l a)^{2 p-1}} \frac{2 \pi^{p}(l a)^{2 p-1}}{(p-1) !} \\
& =\frac{1}{\pi^{-\frac{1}{2}}} \frac{a^{2 p-1}(2 p-1) !}{2^{p-1} 2^{p-1}} \frac{1}{(p-1) !}
\end{aligned}
$$

e

$$
\begin{aligned}
\frac{1}{2} \int_{S_{l \operatorname{sen} \alpha}^{2 p-1}} B\left(\nabla_{1}^{T C_{\alpha} S_{l \operatorname{sen} \alpha}^{2 p-1}}\right) \\
=\sum_{j=0}^{p-1} \frac{1}{j ! \Gamma\left(p-j+\frac{1}{2}\right)} \sum_{h=0}^{j}\left(\begin{array}{l}
j \\
h
\end{array}\right) \frac{(-1)^{h}}{(2(p-j+h)-1) a^{2(j-h)}} \int_{S_{l \operatorname{sen} \alpha}^{2 p-1}} \int^{B} \frac{\mathcal{S}_{1}^{2 p-1}}{4} \\
=\sum_{j=0}^{p-1} \frac{1}{j ! \Gamma\left(p-j+\frac{1}{2}\right)} \sum_{h=0}^{j}\left(\begin{array}{l}
j \\
h
\end{array}\right) \frac{(-1)^{h}}{(2(p-j+h)-1) a^{2(j-h)}} \frac{a^{2 p-1}(2 p-1) !}{\pi^{-\frac{1}{2}} 4^{p}(p-1) !} \\
=\sum_{j=0}^{p-1} \frac{2^{p-j}}{j !(2(p-j)-1) ! !} \sum_{h=0}^{j}\left(\begin{array}{l}
j \\
h
\end{array}\right) \frac{(-1)^{h}}{(2(p-j+h)-1) a^{2(j-h)}} \frac{a^{2 p-1}(2 p-1) !}{4^{p}(p-1) !} .
\end{aligned}
$$

Temos então todos os termos da equação do teorema 10, de uma maneira formal. De fato, a Torção de Reidemeister sobre o cone é calculada na proposição 11 e é igual a

$$
\log \tau\left(C_{\alpha} S_{l \operatorname{sen} \alpha}^{m-1}\right)=\frac{1}{2} \operatorname{Vol}\left(C_{\alpha} S_{l \operatorname{sen} \alpha}^{m-1}\right) .
$$

Comparando com os resultados explícitos do cálculo da Torção Analítica do cone sobre $S^{1}$, $S^{2}$ e $S^{3}$, podemos detectar a contribuição da singularidade. De fato, claramente a fórmula do lema anterior vale para o cone sobre o círculo e sobre a 3-esfera, enquanto uma contribuição da singularidade aparece no caso da esfera. Isto motiva a seguinte conjectura, que será provada no próximo capítulo, 
Conjectura 1. A Torção Analítica do cone $C_{\alpha} S_{l \operatorname{sen} \alpha}^{2 p-1}$ de ângulo $\alpha$ e comprimento $l>0$, sobre a esfera de dimensão ímpar $S^{2 p-1}$, com a métrica induzida pela imersão em $\mathbb{R}^{m+1} e$ condiçôes de contorno absoluta (onde $p>0$ ) é:

$$
\begin{aligned}
& \log T\left(C_{\alpha} S_{l \operatorname{sen} \alpha}^{2 p-1}\right)=\frac{1}{2} \log \operatorname{Vol}\left(C_{\alpha} S_{l \operatorname{sen} \alpha}^{2 p-1}\right) \\
& \quad+\sum_{j=0}^{p-1} \frac{2^{p-j}}{j !(2(p-j)-1) ! !} \sum_{h=0}^{j}\left(\begin{array}{l}
j \\
h
\end{array}\right) \frac{(-1)^{h} \operatorname{sen}^{2(h-j)} \alpha}{(2(p-j+h)-1)} \frac{(2 p-1) ! \operatorname{sen}^{2 p-1} \alpha}{4^{p}(p-1) !} .
\end{aligned}
$$




\title{
Capítulo 6
}

\section{Torção Analítica do cone sobre uma variedade de dimensão ímpar}

\begin{abstract}
Neste capítulo provaremos a conjectura 1. Para isso usaremos o teorema 5. Para obter uma extensão do teorema de Cheeger Müller (teorema 7) para o cone sobre uma variedade Riemanniana fechada de dimensão ímpar, faremos inicialmente um estudo deste caso e depois particularizaremos para o estudo das esferas. Acreditamos que uma prova para caso geral possa ser produzida.
\end{abstract}

\subsection{Cone sobre uma variedade fechada conexa de dimensão ím- par}

Seja $W=C_{l} N$, com dimensão $m+1$, onde $N$ é uma variedade Riemanniana $m$-dimensional fechada e conexa com uma métrica $\tilde{g}$. A métrica de $W$ é da forma

$$
g=d r \otimes d r+r^{2} \tilde{g}
$$

onde as coordenadas para $W$ serão $(r, y)$. Lembramos dos números que estão na equação (1.10),

$$
\alpha_{q}=\frac{1}{2}(1+2 q-m), \quad \mu_{q, n}=\sqrt{\lambda_{q, n}+\alpha_{q}^{2}}
$$

onde $\lambda_{q, n}$ são autovalores de autoformas coexatas $\phi_{n}^{(q)}$ do Laplaciano $\tilde{\Delta}^{(q)}$ de dimensão $q$ na seção.

Lema 11. O espectro positivo do Laplaciano $\Delta^{(q)}$ sobre $W$, com condições de contorno abso- 
luta no bordo, é da forma

$$
\begin{aligned}
\operatorname{Sp} \Delta_{\text {abs }}^{(q)} & =\left\{m_{q, n}: \hat{j}_{\mu_{q, n}, \alpha_{q}, k}^{2} / l^{2}\right\}_{n, k=1}^{\infty} \cup\left\{m_{q-1, n}: \hat{j}_{\mu_{q-1, n}, \alpha_{q-1}, k}^{2} / l^{2}\right\}_{n, k=1}^{\infty} \\
& \cup\left\{m_{q-1, n}: j_{\mu_{q-1, n}, k}^{2} / l^{2}\right\}_{n, k=1}^{\infty} \cup\left\{m_{q-2, n}: j_{\mu_{q-2, n}, k}^{2} / l^{2}\right\}_{n, k=1}^{\infty} \\
& \cup\left\{m_{q, 0}: \hat{j}_{\left|\alpha_{q}\right|, \alpha_{q}, k}^{2} / l^{2}\right\}_{k=1}^{\infty} \cup\left\{m_{q-1,0}: \hat{j}_{\left|\alpha_{q-1}\right|, \alpha_{q-1}, k}^{2} / l^{2}\right\}_{k=1}^{\infty} .
\end{aligned}
$$

No caso de condições de contorno relativa no bordo temos

$$
\begin{aligned}
\operatorname{Sp} \Delta_{\text {rel }}^{(q)} & =\left\{m_{q, n}: j_{\mu_{q, n}, k}^{2} / l^{2}\right\}_{n, k=1}^{\infty} \cup\left\{m_{q-1, n}: j_{\mu_{q-1, n}, k}^{2} / l^{2}\right\}_{n, k=1}^{\infty} \\
& \cup\left\{m_{q-1, n}: \hat{j}_{\mu_{q-1, n},-\alpha_{q-1}, k}^{2} / l^{2}\right\}_{n, k=1}^{\infty} \cup\left\{m_{q-2, n}: \hat{j}_{\mu_{q-2, n},-\alpha_{q-2}, k}^{2} / l^{2}\right\}_{n, k=1}^{\infty} \\
& \cup\left\{m_{q, 0}: j_{\left|\alpha_{q}\right|, k}^{2} / l^{2}\right\}_{k=1}^{\infty} \cup\left\{m_{q-1,0}: j_{\left|\alpha_{q-1}\right|, k}^{2} / l^{2}\right\}_{k=1}^{\infty},
\end{aligned}
$$

onde $\hat{j}_{\nu, c, k}$ é o zero da função $\hat{J}_{\nu, c}(r)=c J_{\nu}(r)+r J_{\nu}^{\prime}(r), j_{\nu, k}$ é o zero da função de Bessel $J_{\nu}(x)$ e $m_{q, n}$ é a multiplicidade do autovalor $\lambda_{q, n}$.

Demonstração. Pelo lema 2 e o teorema 3 temos um sistema completo de soluções quadrado integráveis da equação do Laplaciano $\Delta^{(q)} u=\lambda u$, com $\lambda \neq 0$. Como o espectro de $\Delta^{(q)}$ é pontual, para obter uma resolução discreta (mais precisamente a parte positiva) de $\Delta^{(q)}$, temos que determinar quais destas soluções satisfazem as condições de contorno absoluta (relativa) em $r=l$. Assim consideramos as formas do tipo 1, 2, 3, 4, E, $O$ e aplicando as condições de contorno absoluta (2.6) e relativa (2.7) obtemos o resultado. Desta maneira, determinaremos apenas os autovalores para o caso com condições de contorno absoluta no bordo, já que o caso relativo segue de maneira análoga.

Lembramos que,

$$
\mathcal{B}_{\text {abs }}(\omega)=0 \quad \text { se e somente se } \quad\left\{\begin{array}{l}
\left.\omega_{\text {norm }}\right|_{\partial W}=0 \\
\left.(d \omega)_{\text {norm }}\right|_{\partial W}=0 .
\end{array}\right.
$$

Aplicando este sistema na forma do tipo 1 obtemos

$$
\left.\partial_{r}\left(\psi_{1, n}^{(q)}\right)\right|_{r=l}=0 \Rightarrow \alpha_{q} J_{\mu_{q, n}}(\lambda l)+\lambda l J_{\mu_{q, n}}^{\prime}(\lambda l)=0 .
$$

Logo $\lambda=\hat{j}_{\mu_{q, n}, \alpha_{q}, k} / l$.

No caso da forma do tipo 2 temos

$$
\left.\partial_{r}\left(\psi_{2, n}^{(q)}\right)\right|_{r=l}=0 \Rightarrow \alpha_{q-1} J_{\mu_{q-1, n}}(\lambda l)+\lambda l J_{\mu_{q-1, n}}^{\prime}(\lambda l)=0
$$

e $\operatorname{assim} \lambda=\hat{j}_{\mu_{n, q-1}, \alpha_{q-1}, k} / l$.

Para o tipo 3 temos o sistema

$$
\left\{\begin{array}{l}
\left(r^{\alpha_{q-1}-1} J_{\mu_{q-1, n}}(\lambda r)\right)_{r=l}=0, \\
\left.\left(\partial_{r}\left(r^{2 \alpha_{q-1}+1} \partial_{r}\left(r^{-\alpha_{q-1}} J_{\mu_{q-1, n}}(\lambda r)\right)\right)-\lambda_{q-1, n} r^{\alpha_{q-1}-1} J_{\mu_{q-1, n}}(\lambda r)\right)\right|_{r=l}=0,
\end{array}\right.
$$


e ainda

$$
\begin{aligned}
& \partial_{r}\left(r^{2 \alpha_{q-1}+1} \partial_{r}\left(r^{-\alpha_{q-1}} J_{\mu_{q-1, n}}(\lambda r)\right)\right)= \\
& =\partial_{r}\left(-\alpha_{q-1} r^{\alpha_{q-1}} J_{\mu_{q-1, n}}(\lambda r)+\lambda r^{\alpha_{q-1}+1} J_{\mu_{q-1, n}}^{\prime}(\lambda r)\right) \\
& =-\alpha_{q-1}^{2} r^{\alpha_{q-1}-1} J_{\mu_{q-1, n}}(\lambda r)+\lambda r^{\alpha_{q-1}} J_{\mu_{q-1, n}}^{\prime}(\lambda r)+\lambda^{2} r^{\alpha_{q-1}+1} J_{\mu_{q-1, n}}^{\prime \prime}(\lambda r) .
\end{aligned}
$$

Agora usando relações clássicas entre as funções de Bessel e a sua derivada, obtemos que

$$
z^{2} J_{\nu}^{\prime \prime}(z)=-z^{2} J_{\nu}(z)+\nu^{2} J_{\nu}(z)-z J_{\nu}^{\prime}(z)
$$

Substituindo $\lambda^{2} r^{2} J_{\mu_{q-1, n}}^{\prime \prime}(\lambda r)$ na equação (6.1) obtemos

$$
\begin{aligned}
& \partial_{r}\left(r^{2 \alpha_{q-1}+1} \partial_{r}\left(r^{-\alpha_{q-1}} J_{\mu_{q-1, n}}(\lambda r)\right)\right)= \\
& =-\alpha_{q-1}^{2} r^{\alpha_{q-1}-1} J_{\mu_{q-1, n}}(\lambda r)-\lambda^{2} r^{\alpha_{q-1}+1} J_{\mu_{q-1, n}}(\lambda r)+r^{\alpha_{q-1}} \mu_{q-1, n}^{2} J_{\mu_{q-1, n}}(\lambda r) .
\end{aligned}
$$

Com a solução da primeira equação do sistema e a equação acima, concluímos que $\lambda=$ $j_{\mu_{q-1, n}, k} / l$.

No tipo 4 temos

$$
\left.\left(r^{\alpha_{q-2}+1} J_{\mu_{q-2, n}, k}(\lambda l)\right)\right|_{r=l}=0 \Rightarrow \lambda=\frac{j_{\mu_{q-2, n}, k}}{l} .
$$

Da mesma forma que as anteriores, nos tipos $E$ e $O$ temos $\lambda=\hat{j}_{\left|\alpha_{q}\right|, \alpha_{q}, k} / l$ e $\lambda=$ $\hat{j}_{\left|\alpha_{q-1}\right|, \alpha_{q-1}, k} / l$, respectivamente. E o resultado segue.

Com este lema é possível provar um tipo de "Dualidade de Poincaré" para a Torção Analítica no caso de $C_{l} N$.

Teorema 18. Seja $N$ uma variedade Riemanniana fechada orientável de dimensão $m$ e seja $W=C_{l} N$. Então

$$
\log T_{\text {abs }}(W)=(-1)^{m} \log T_{\text {rel }}(W) .
$$

Demonstração. Usando a dualidade de Hodge, dada por meio de $*$, temos que as formas do tipo $1,2,3,4, E$ e $O$ são levadas por $*$ nas formas do tipo 4, 3, 2, 1, $O$ e $E$, respectivamente. $\mathrm{E}$ ainda, * leva uma forma de dimensão $q$, que satisfaz condições de contorno absoluta no bordo, em uma forma de dimensão $m+1-q$, que satisfaz as condições de contorno relativa no bordo. Logo, pelo lema anterior, $\operatorname{Sp} \Delta_{\text {abs }}^{(q)}=\operatorname{Sp} \Delta_{\text {rel }}^{(m+1-q)}$. Comparando a definição das formas no teorema 3 com o lema 1 temos que as formas do tipo 1, 3 e $E$ são coexatas e as formas do tipo 2, 4 e $O$ são exatas, o operador $d$ leva formas do tipo 1, 3 e $E$ em formas do tipo 2, 4 e $O$, respectivamente, enquanto $d^{\dagger}$ faz o processo inverso. Então defina

$$
\begin{aligned}
F_{c f, \text { abs }}^{(q)}: & =\left\{m_{q, n}: \hat{j}_{\mu_{q, n}, \alpha_{q}, k}^{2} / l^{2}\right\}_{n, k=1}^{\infty} \cup\left\{m_{q-1, n}: j_{\mu_{q-1, n}, k}^{2} / l^{2}\right\}_{n, k=1}^{\infty} \\
& \cup\left\{m_{q, 0}: \hat{j}_{\left|\alpha_{q}\right|, \alpha_{q}, k}^{2} / l^{2}\right\}_{k=1}^{\infty} .
\end{aligned}
$$


$F_{c f \text {,abs }}^{(q)}$ é a união dos autovalores de dimensão $q$ das formas cofechadas com condições de contorno absoluta. Defina

$$
\begin{aligned}
F_{f, \mathrm{abs}}^{(q)}: & =\left\{m_{q-1, n}: \hat{j}_{\mu_{q-1, n}, \alpha_{q-1}, k}^{2} / l^{2}\right\}_{n, k=1}^{\infty} \cup\left\{m_{q-2, n}: j_{\mu_{q-2, n}, k}^{2} / l^{2}\right\}_{n, k=1}^{\infty} \\
& \cup\left\{m_{q-1,0}: \hat{j}_{\left|\alpha_{q-1}\right|, \alpha_{q-1}, k}^{2} / l^{2}\right\}_{k=1}^{\infty} .
\end{aligned}
$$

$F_{f \text {,abs }}^{(q)}$ é a união dos autovalores das formas fechadas de dimensão $q$ com condições de contorno absoluta. Claramente $\operatorname{Sp} \Delta_{\text {abs }}^{(q)}=F_{c f \text {,abs }}^{(q)} \cup F_{f \text {,abs }}^{(q)}$ e $F_{c f, \text { abs }}^{(q)}=F_{f, \text { abs }}^{(q+1)}$. Então

$$
\begin{aligned}
t_{\mathrm{abs}}(s) & =\frac{1}{2} \sum_{q=0}^{m+1}(-1)^{q} q \zeta\left(s, \Delta_{\mathrm{abs}}^{(q)}\right)=\frac{1}{2} \sum_{q=0}^{m+1}(-1)^{q} q \zeta\left(s, \Delta_{\mathrm{rel}}^{(m+1-q)}\right) \\
& =(-1)^{m} t_{\mathrm{rel}}(s)+\frac{(m+1)}{2} \sum_{q=0}^{m+1}(-1)^{m+1-q} \zeta\left(s, \Delta_{\mathrm{rel}}^{(q)}\right) \\
& =(-1)^{m} t_{\mathrm{rel}}(s)+\frac{(m+1)}{2} \sum_{q=0}^{m+1}(-1)^{q}\left(\zeta\left(s, F_{f, \text { abs }}^{(q+1)}\right)+\zeta\left(s, F_{f, \mathrm{abs}}^{(q)}\right)\right) \\
& =(-1)^{m} t_{\mathrm{rel}}(s) .
\end{aligned}
$$

Agora, $\log T_{\text {abs }}(W)=t_{\text {abs }}^{\prime}(0)=(-1)^{m} t_{\text {rel }}^{\prime}(0)=(-1)^{m} \log T_{\text {rel }}(W)$.

A partir de agora consideramos $\operatorname{dim} N=2 p-1$ e que a condição de contorno sobre o bordo de $W$ será sempre a condição de contorno absoluta $\left(\mathcal{B}_{\text {abs }}\right)$. Desta forma, não faremos mais referências a estes fatos. Usando os autovalores do Laplaciano de $W$ (lema 11), defina $t(s)$ como

$$
t(s)=\frac{1}{2} \sum_{q=1}^{2 p}(-1)^{q} q \zeta\left(s, \Delta^{(q)}\right) .
$$

Então, $\log T_{\text {abs }}(W)=t^{\prime}(0)$.

Lema 12. Se $t(s)$ é como acima, então

$$
\begin{aligned}
t(s)= & \frac{l^{2 s}}{2} \sum_{q=0}^{p-2}(-1)^{q}\left(\sum_{n, k=1}^{\infty} m_{q, n}\left(2 j_{\mu_{q, n}, k}^{-2 s}-\hat{j}_{\mu_{q, n}, \alpha_{q}, k}^{-2 s}-\hat{j}_{\mu_{q, n},-\alpha_{q}, k}^{-2 s}\right)\right) \\
& +(-1)^{p-1} \frac{l^{2 s}}{2}\left(\sum_{n, k=1}^{\infty} m_{p-1, n}\left(j_{\mu_{p-1, n}, k}^{-2 s}-\left(j_{\mu_{p-1, n}, k}^{\prime}\right)^{-2 s}\right)\right) \\
& -\frac{l^{2 s}}{2} \sum_{q=0}^{p-1}(-1)^{q} \operatorname{rk} \mathcal{H}_{q}(N ; \mathbb{R}) \sum_{k=1}^{\infty}\left(j_{-\alpha_{q-1}, k}^{-2 s}-j_{-\alpha_{q}, k}^{-2 s}\right) .
\end{aligned}
$$


Demonstração. Usando o lema 11, temos

$$
\begin{aligned}
l^{2 s} \zeta\left(s, \Delta^{(q)}\right)= & \sum_{n, k=1}^{\infty} m_{q, n} \hat{j}_{\mu_{q, n}, \alpha_{q}, k}^{-2 s}+\sum_{n, k=1}^{\infty} m_{q-1, n} \hat{j}_{\mu_{q-1, n}, \alpha_{q-1}, k}^{-2 s} \\
& +\sum_{n, k=1}^{\infty} m_{q-1, n} j_{\mu_{q-1, n}, k}^{-2 s}+\sum_{n, k=1}^{\infty} m_{q-2, n} j_{\mu_{q-2, n}, k}^{-2 s} \\
& +\sum_{k=1}^{\infty} m_{q, 0} \hat{j}_{\left|\alpha_{q}\right|, \alpha_{q}, k}^{-2 s}+\sum_{k=1}^{\infty} m_{q-1,0} \hat{j}_{\left|\alpha_{q-1}\right|, \alpha_{q-1}, k}^{-2 s} .
\end{aligned}
$$

Para $q$ fixo, $0 \leq q \leq 2 p-2$, temos termos seqüenciais. Somando tais termos, obtemos

$$
\begin{aligned}
& (-1)^{q} q \sum_{n, k=1}^{\infty} m_{q, n} \hat{j}_{\mu_{q, n}, \alpha_{q}, k}^{-2 s}+(-1)^{q+1}(q+1) \sum_{n, k=1}^{\infty} \hat{m}_{q, n} \hat{j}_{\mu_{q, n}, \alpha_{q}, k}^{-2 s} \\
& +(-1)^{q+1}(q+1) \sum_{n, k=1}^{\infty} m_{q, n} j_{\mu_{q, n}, k}^{-2 s}+(-1)^{q+2}(q+2) \sum_{n, k=1}^{\infty} m_{q, n} j_{\mu_{q, n}, k}^{-2 s} \\
& +q(-1)^{q} \sum_{k=1}^{\infty} m_{q, 0} \hat{j}_{\left|\alpha_{q}\right|, \alpha_{q}, k}^{-2 s}+(q+1)(-1)^{q+1} \sum_{k=1}^{\infty} m_{q, 0} \hat{j}_{\left|\alpha_{q}\right|, \alpha_{q}, k}^{-2 s} \\
& =(-1)^{q}\left(\sum_{n, k=1}^{\infty} m_{q, n} j_{\mu_{q, n}, k}^{-2 s}-\sum_{n, k=1}^{\infty} m_{q, n} \hat{j}_{\mu_{q, n}, \alpha_{q}, k}^{-2 s}\right)+(-1)^{q+1} \sum_{k=1}^{\infty} m_{q, 0} \hat{j}_{\left|\alpha_{q}\right|, \alpha_{q}, k}^{-2 s} .
\end{aligned}
$$

Segue que $t(s)$ é da forma,

$$
t(s)=\frac{l^{2 s}}{2} \sum_{q=0}^{2 p-2}(-1)^{q} \sum_{n, k=1}^{\infty} m_{q, n}\left(j_{\mu_{q, n}, k}^{-2 s}-\hat{j}_{\mu_{q, n}, \alpha_{q}, k}^{-2 s}\right)+\frac{l^{2 s}}{2} \sum_{q=0}^{2 p-1}(-1)^{q+1} \sum_{k=1}^{\infty} m_{q, 0} \hat{j}_{\left|\alpha_{q}\right|, \alpha_{q}, k}^{-2 s} .
$$

Observamos que $\lambda_{q, n}=\lambda_{2 p-2-q, n}$, por meio da isometria na equação (2.12), e ainda $\alpha_{q}=\frac{1}{2}(1+2 q-2 p+1)=q-p+1=-\alpha_{2 p-2-q}, \operatorname{logo} \mu_{q, n}=\mu_{2 p-2-q, n}$. Desta forma, fixando $q, 0 \leq q \leq p-2$, e olhando na primeira parte da soma anterior, vemos que

$$
\begin{aligned}
& (-1)^{q} \sum_{n, k=1}^{\infty} m_{q, n}\left(j_{\mu_{q, n}, k}^{-2 s}-\hat{j}_{\mu_{q, n}, \alpha_{q}, k}^{-2 s}\right)+(-1)^{(2 p-2-q)} \sum_{n, k=1}^{\infty} m_{q, n}\left(j_{\mu_{q, n}, k}^{-2 s}-\hat{j}_{\mu_{q, n},-\alpha_{q}, k}^{-2 s}\right)= \\
& =(-1)^{q} \sum_{n, k=1}^{\infty} m_{q, n}\left(2 j_{\mu_{q, n}, k}^{-2 s}-\hat{j}_{\mu_{q, n}, \alpha_{q}, k}^{-2 s}-\hat{j}_{\mu_{q, n},-\alpha_{q}, k}^{-2 s}\right),
\end{aligned}
$$

observe que, quando $q=p-1$, tem-se $\lambda_{p-1, n}=\lambda_{2 p-1-(p-1), n}$ e $\alpha_{q}$ identicamente nulo, portanto, não temos uma soma como acima. Logo,

$$
\begin{aligned}
t(s) & =\frac{l^{2 s}}{2} \sum_{q=0}^{p-2}(-1)^{q} \sum_{n, k=1}^{\infty} m_{q, n}\left(2 j_{\mu_{q, n}, k}^{-2 s}-\hat{j}_{\mu_{q, n}, \alpha_{q}, k}^{-2 s}-\hat{j}_{\mu_{q, n},-\alpha_{q}, k}^{-2 s}\right) \\
& +(-1)^{p-1} \frac{l^{2 s}}{2} \sum_{n, k=1}^{\infty} m_{p-1, n}\left(j_{\mu_{p-1, n}, k}^{-2 s}-\left(j_{\mu_{p-1, n}, k}^{\prime}\right)^{-2 s}\right) \\
& +\frac{l^{2 s}}{2} \sum_{q=0}^{2 p-1}(-1)^{q+1} \sum_{k=1}^{\infty} m_{q, 0} \hat{j}_{\left|\alpha_{q}\right|, \alpha_{q}, k}^{-2 s} .
\end{aligned}
$$


Falta apenas estudar a soma

$$
\sum_{q=0}^{2 p-1}(-1)^{q+1} \sum_{k=1}^{\infty} m_{q, 0} \hat{j}_{\left|\alpha_{q}\right|, \alpha_{q}, k}^{-2 s}
$$

Para isso, precisamos de algumas propriedades das funções de Bessel que podem ser encontradas em [48]. Como $\operatorname{dim} N=2 p-1$, temos que $\alpha_{q}$ é um número inteiro igual a $q-p+1$, e ainda, este valor é negativo para $0 \leq q<p-1$. Fixe $q, 0 \leq q<p-1$, e considere a função $\hat{J}_{-\alpha_{q}, \alpha_{q}}(z)=\alpha_{q} J_{-\alpha_{q}}(z)+z J_{-\alpha_{q}}^{\prime}(z)$. Utilizando a identidade

$$
z J_{\mu}^{\prime}(z)=-z J_{\mu+1}(z)+\mu J_{\mu}(z)
$$

obtemos $\hat{J}_{-\alpha_{q}, \alpha_{q}}(z)=-z J_{-\alpha_{q}+1}(z)=-z J_{-\alpha_{q-1}}(z)$ e assim $\hat{j}_{\left|\alpha_{q}\right|, k, \alpha_{q}}=j_{-\alpha_{q-1}, k}$. Agora fixe $q, p-1<q \leq 2 p-1$, assim $\alpha_{q}$ é positivo e como

$$
z J_{\mu}^{\prime}(z)=z J_{\mu-1}(z)-\mu J_{\mu}(z)
$$

a função $\hat{J}_{\alpha_{q}, \alpha_{q}}(z)=\alpha_{q} J_{\alpha_{q}}(z)+z J_{\alpha_{q}}^{\prime}(z)$ é igual a $z J_{\alpha_{q}-1}(z)$, e então $\hat{j}_{\left|\alpha_{q}\right|, k, \alpha_{q}}=j_{\alpha_{q-1}, k}$. Note que, quando $q=p-1$, tem-se $\alpha_{p-1}=0$ e, então, $j_{\alpha_{p-1}, k, \alpha_{p-1}}=j_{0, k}^{\prime}=j_{1, k}$. Logo,

$$
\begin{aligned}
\sum_{q=0}^{2 p-1}(-1)^{q+1} & \sum_{k=1}^{\infty} m_{q, 0} \hat{j}_{\left|\alpha_{q}\right|, \alpha_{q}, k}^{-2 s}= \\
& =\sum_{q=0}^{p-2}(-1)^{q+1} \sum_{k=1}^{\infty} \frac{m_{q, 0}}{j_{-\alpha_{q-1}, k}^{-2 s}}+(-1)^{p} \sum_{k=1}^{\infty} \frac{m_{p-1,0}}{j_{1, k}^{-2 s}}+\sum_{q=p}^{2 p-1}(-1)^{q+1} \sum_{k=1}^{\infty} \frac{m_{q, 0}}{j_{\alpha_{q-1}, k}^{-2 s}} .
\end{aligned}
$$

Utilizando a dualidade dada por $*$ tem-se $m_{q, 0}=m_{2 p-1-q, 0}$. Assim, reduzimos a soma acima,

$$
\begin{aligned}
& \sum_{q=0}^{2 p-1}(-1)^{q+1} \sum_{k=1}^{\infty} m_{q, 0} \hat{j}_{\left|\alpha_{q}\right|, k}^{-2 s}=\sum_{q=0}^{p-2}(-1)^{q+1} \sum_{k=1}^{\infty} m_{q, 0} j_{-\alpha_{q-1}, k}^{-2 s} \\
& \quad+(-1)^{p} \sum_{k=1}^{\infty} m_{p-1,0} j_{1, k}^{-2 s}+\sum_{q=0}^{p-1}(-1)^{2 p-q} \sum_{k=1}^{\infty} m_{2 p-1-q, 0} j_{\alpha_{2 p-2-q}, k}^{-2 s} \\
& \quad=\sum_{q=0}^{p-2}(-1)^{q+1} \sum_{k=1}^{\infty} m_{q, 0} j_{-\alpha_{q-1}, k}^{-2 s}+(-1)^{p} \sum_{k=1}^{\infty} m_{p-1,0} j_{1, k}^{-2 s}+\sum_{q=0}^{p-1}(-1)^{q} \sum_{k=1}^{\infty} m_{q, 0} j_{-\alpha_{q}, k}^{2 s} \\
& \quad=\sum_{q=0}^{p-1}(-1)^{q+1} m_{q, 0} \sum_{k=1}^{\infty} j_{-\alpha_{q-1}, k}^{-2 s}-j_{-\alpha_{q}, k}^{-2 s} .
\end{aligned}
$$


Note que $m_{q, 0}=\operatorname{rk} H_{q}(N, \mathbb{R})$, por meio do teorema de de Rham, e a fórmula para $t(s)$ fica

$$
\begin{aligned}
t(s)= & \frac{l^{2 s}}{2} \sum_{q=0}^{p-2}(-1)^{q}\left(\sum_{n, k=1}^{\infty} m_{q, n}\left(2 j_{\mu_{q, n}, k}^{-2 s}-\hat{j}_{\mu_{q, n}, \alpha_{q}, k}^{-2 s}-\hat{j}_{\mu_{q, n},-\alpha_{q}, k}^{-2 s}\right)\right) \\
& +(-1)^{p-1} \frac{l^{2 s}}{2}\left(\sum_{n, k=1}^{\infty} m_{p-1, n}\left(j_{\mu_{p-1, n}, k}^{-2 s}-\left(j_{\mu_{p-1, n}, k}^{\prime}\right)^{-2 s}\right)\right) \\
& -\frac{l^{2 s}}{2} \sum_{q=0}^{p-1}(-1)^{q} \mathrm{rk} \mathcal{H}_{q}(N ; \mathbb{R}) \sum_{k=1}^{\infty}\left(j_{-\alpha_{q-1}, k}^{-2 s}-j_{-\alpha_{q}, k}^{-2 s}\right) .
\end{aligned}
$$

Defina as funções,

$$
\begin{aligned}
Z_{q}(s) & =\sum_{n, k=1}^{\infty} m_{q, n} j_{\mu_{q, n}, k}^{-2 s}, & \dot{Z}_{q}(s) & =\sum_{n, k=1}^{\infty} m_{q, n}\left(j_{\mu_{q, n}, k}^{\prime}\right)^{-2 s}, \\
Z_{q, \pm}(s) & =\sum_{n, k=1}^{\infty} m_{q, n} \hat{j}_{\mu_{q, n}, \pm \alpha_{q}, k}^{-2 s}, & z_{q}(s) & =\sum_{k=1}^{\infty}\left(j_{-\alpha_{q-1}, k}^{-2 s}-j_{-\alpha_{q}, k}^{-2 s}\right)
\end{aligned}
$$

para $0 \leq q \leq p-1$, e

$$
\begin{array}{rlr}
t_{q}(s) & =2 Z_{q}(s)-Z_{q,+}(s)-Z_{q,-}(s), & 0 \leq q \leq p-2 \\
t_{p-1}(s) & =Z_{p-1}(s)-\dot{Z}_{p-1}(s) .
\end{array}
$$

Então,

$$
\begin{aligned}
t(s)= & \frac{l^{2 s}}{2} \sum_{q=0}^{p-2}(-1)^{q}\left(2 Z_{q}(s)-Z_{q,+}(s)-Z_{q,-}(s)\right)+(-1)^{p-1} \frac{l^{2 s}}{2}\left(Z_{q-1}(s)-\dot{Z}_{q-1}(s)\right) \\
& -\frac{l^{2 s}}{2} \sum_{q=0}^{p-1}(-1)^{q} \operatorname{rk} \mathcal{H}_{q}(N ; \mathbb{R}) z_{q}(s) \\
= & \frac{l^{2 s}}{2} \sum_{q=0}^{p-1}(-1)^{q} t_{q}(s)-\frac{l^{2 s}}{2} \sum_{q=0}^{p-1}(-1)^{q} \operatorname{rk} \mathcal{H}_{q}(N ; \mathbb{R}) z_{q}(s),
\end{aligned}
$$

$\mathrm{e}$

$$
\begin{aligned}
\log T(W)=t^{\prime}(0)= & \frac{\log l^{2}}{2}\left(\sum_{q=0}^{p-1}(-1)^{q} t_{q}(0)+\sum_{q=0}^{p-1}(-1)^{q+1} \mathrm{rk} H_{q}(N, \mathbb{R}) z_{q}(0)\right) \\
& +\frac{1}{2}\left(\sum_{q=0}^{p-1}(-1)^{q} t_{q}^{\prime}(0)+\sum_{q=0}^{p-1}(-1)^{q+1} \mathrm{rk} H_{q}(N, \mathbb{R}) z_{q}^{\prime}(0)\right) .
\end{aligned}
$$




\subsection{Estudo de $t_{p-1}(s)$}

Nesta seção, trabalharemos com as seqüências duplas $S_{p-1}=\left\{m_{p-1, n}: j_{\mu_{p-1, n}, k}^{2}\right\}_{n=1}^{\infty}$ e $\dot{S}_{p-1}=$ $\left\{m_{p-1, n}:\left(j_{\mu_{p-1, n}, k}^{\prime}\right)^{2}\right\}_{n=1}^{\infty}$, já que $Z_{p-1}(s)=\zeta\left(s, S_{p-1}\right)$ e $\dot{Z}_{p-1}(s)=\zeta\left(s, \dot{S}_{p-1}\right)$.

Primeiro, verificaremos a definição [13, ou seja, que $S_{p-1}$ e $\dot{S}_{p-1}$ são espectralmente decompostas sobre $U_{p-1}$. Considere a seqüência simples $U_{p-1}=\left\{m_{p-1, n}: \mu_{p-1, n}\right\}_{n=1}^{\infty}$.

Lema 13. A seqüência $U_{p-1}$ é uma seqüência totalmente regular do tipo espectral com ordem infinita, $e\left(U_{p-1}\right)=g\left(U_{p-1}\right)=2 p-1, e$

$$
\zeta\left(s, U_{p-1}\right)=\zeta\left(\frac{s}{2}, \tilde{U}_{p-1}\right)
$$

onde $\tilde{U}_{p-1}=\left\{m_{p-1, n}: \lambda_{p-1, n}\right\}$. Além disso, a função $\zeta\left(s, U_{p-1}\right)$ possui polos simples em $s=2(p-k)-1$, para $k=0,1,2, \ldots$

Demonstração. Primeiro lembraremos alguns fatos a respeito dos autovalores $\lambda_{q, n}$, do Laplaciano de dimensão $q$ de uma variedade Riemanniana compacta sem bordo $M$. Todas estas afirmações podem ser encontradas em [20], seção 1.12. Para $n$ suficientemente grande, $\lambda_{q, n}$ se comporta como $n \frac{2}{\operatorname{dim} M}$, ou seja, $\lambda_{q, n} \sim n \frac{2}{\operatorname{dim} M}$ e segundo a função $\zeta\left(s, \operatorname{Sp}_{+} \Delta_{M}^{(q)}\right)$ possui polos simples em $s=\frac{\operatorname{dim} M-k}{2}, k=0,1,2, \ldots$ E ainda $\operatorname{Sp}_{+} \Delta_{M}^{(q)}$ é uma seqüência totalmente regular do tipo espectral com ordem infinita.

Para mostrar que e $\left(U_{p-1}\right)=\mathrm{g}\left(U_{p-1}\right)=2 p-1$, temos a seqüência de identidades

$$
\mathrm{e}\left(U_{p-1}\right)=\limsup _{n \rightarrow \infty} \frac{\log n}{\log \left|\lambda_{q, n}^{\frac{1}{2}}\right|}=\limsup _{n \rightarrow \infty} \frac{\log n}{\frac{1}{2 p-1} \log n}=2 p-1
$$

Para a representação de $\zeta\left(s, U_{p-1}\right)$ como acima temos,

$$
\zeta\left(s, U_{p-1}\right)=\zeta\left(\frac{s}{2}, \tilde{U}_{p-1}\right)
$$

onde

$$
\zeta\left(\frac{s}{2}, \tilde{U}_{p-1}\right)=\sum_{n=1}^{\infty} \frac{m_{p-1, n}}{\lambda_{p-1, n}^{\frac{s}{2}}}
$$

o que segue direto da definição de $\zeta\left(s, U_{p-1}\right)$. Logo, com a observação a respeito dos polos de $\zeta\left(s, \tilde{U}_{p-1}\right)$ no início da prova temos que $\zeta\left(s, U_{p-1}\right)$ possui polos simples em $s=2(p-k)-1$, para $k=0,1,2, \ldots$.

Lema 14. O logaritmo da função Gamma associada à seqüência $S_{p-1, n} / \mu_{p-1}^{2}$ e o logaritmo da função Gamma associada à seqüência $\dot{S}_{p-1, n} / \mu_{p-1}$ possuem as representaçôes a seguir, 
com $\lambda \in D_{\theta_{1}, c_{1}}$, respectivamente,

$$
\begin{aligned}
\log \Gamma\left(-\lambda, S_{p-1, n} / \mu_{p-1, n}^{2}\right)= & -\log \prod_{k=1}^{\infty}\left(1+\frac{(-\lambda) \mu_{p-1, n}^{2}}{j_{\mu_{p-1, n}, k}^{2}}\right) \\
= & -\log I_{\mu_{p-1, n}}\left(\mu_{p-1, n} \sqrt{-\lambda}\right)+\left(\mu_{p-1, n}\right) \log \sqrt{-\lambda} \\
& +\mu_{p-1, n} \log \left(\mu_{p-1, n}\right)-\mu_{p-1, n} \log 2-\log \Gamma\left(\mu_{p-1, n}+1\right), \\
\log \Gamma\left(-\lambda, \dot{S}_{p-1, n} / \mu_{p-1, n}^{2}\right)= & -\log \prod_{k=1}^{\infty}\left(1+\frac{(-\lambda)\left(\mu_{p-1, n}\right)^{2}}{\left(j_{\mu_{p-1, n}, k}^{\prime}\right)^{2}}\right) \\
= & -\log I_{\mu_{p-1, n}}^{\prime}\left(\mu_{p-1, n} \sqrt{-\lambda}\right)+\left(\mu_{p-1, n}-1\right) \log \sqrt{-\lambda} \\
& +\mu_{p-1, n} \log \left(\mu_{p-1, n}\right)-\mu_{p-1, n} \log 2-\log \Gamma\left(\mu_{p-1, n}+1\right) .
\end{aligned}
$$

Demonstração. Notamos primeiro que $S_{p-1, n}$ e $\dot{S}_{p-1, n}$ são seqüências de números reais positivos, logo para escolha do domínio de $\lambda$ é suficiente fixar $\theta_{1} \in(0, \pi)$ e considerar $c_{1}=$ $\frac{1}{2} \min \left(j_{\mu_{p-1,1}}^{2},\left(j_{\mu_{p-1,1}}^{\prime}\right)^{2}\right)$.

Lembremos as equações (4.26), onde temos as seguintes representações,

$$
I_{\nu}(z)=\frac{z^{\nu}}{2^{\nu} \Gamma(\nu+1)} \prod_{k=1}^{\infty}\left(1+\frac{z^{2}}{j_{\nu, k}^{2}}\right), \quad I_{\nu}^{\prime}(z)=\frac{z^{\nu-1}}{2^{\nu} \Gamma(\nu)} \prod_{k=1}^{\infty}\left(1+\frac{z^{2}}{\left(j_{\nu, k}^{\prime}\right)^{2}}\right) .
$$

Usando estas representações, obtemos as representações para a função Gamma associada as seqüências $S_{p-1, n} / \mu_{p-1}^{2}$ e $\dot{S}_{p-1, n} / \mu_{p-1}$ sem maiores problemas. E o lema segue.

Com este lema temos uma expansão assintótica completa para $\lambda$ grande em $D_{\theta_{1}, c_{1}}$ das funções Gamma, $\log \Gamma\left(-\lambda, S_{p-1, n} /\left(\mu_{p-1, n}\right)^{2}\right)$ e $\log \Gamma\left(-\lambda, \dot{S}_{p-1, n} /\left(\mu_{p-1, n}\right)^{2}\right)$. Desta maneira $S_{p-1, n} /\left(\mu_{p-1, n}\right)^{2}$ e $\dot{S}_{p-1, n} /\left(\mu_{p-1, n}\right)^{2}$ são seqüências do tipo espectral (ver definição 2.5 de [46]), mais ainda são seqüências totalmente regulares do tipo espectral com ordem infinita.

Lema 15. A diferença entre o logaritmo da função Gamma associada à $S_{p-1, n} / \mu_{p-1}^{2}$ e o logaritmo da função Gamma associada à $\dot{S}_{p-1, n} / \mu_{p-1}^{2}$ possui a expansão assintótica uniforme a seguir, para $n$ suficientemente grande e $\lambda \in D_{\theta_{1}, c_{1}}$, respectivamente,

$$
\begin{aligned}
\log \Gamma\left(-\lambda, S_{p-1, n} /\left(\mu_{p-1, n}\right)^{2}\right)-\log \Gamma\left(-\lambda, \dot{S}_{p-1, n} /\left(\mu_{p-1, n}\right)^{2}\right)= \\
=-\log I\left(\mu_{p-1, n} \sqrt{-\lambda}\right)+\log I^{\prime}\left(\mu_{p-1, n} \sqrt{-\lambda}\right)+\log \sqrt{-\lambda} \\
=\frac{1}{2} \log (1-\lambda)+\sum_{j=1}^{2 p-1} \phi_{p-1, j}(\lambda) \frac{1}{\mu_{p-1, n}^{j}}+O\left(\frac{1}{\mu_{p-1, n}^{2 p}}\right) .
\end{aligned}
$$

Demonstração. Considerando as representações do lema 14 e fazendo a diferença entre os logaritmos das funções Gamma, $\log \Gamma\left(-\lambda, S_{p-1, n} /\left(\mu_{p-1, n}\right)^{2}\right)$ e $\log \Gamma\left(-\lambda, \dot{S}_{p-1, n} /\left(\mu_{p-1, n}\right)^{2}\right)$, obtemos

$$
\begin{aligned}
\log \Gamma\left(-\lambda, S_{p-1, n} /\left(\mu_{p-1, n}\right)^{2}\right)-\log \Gamma\left(-\lambda, \dot{S}_{p-1, n} /\left(\mu_{p-1, n}\right)^{2}\right)= \\
\quad=-\log I\left(\mu_{p-1, n} \sqrt{-\lambda}\right)+\log I^{\prime}\left(\mu_{p-1, n} \sqrt{-\lambda}\right)+\log \sqrt{-\lambda}
\end{aligned}
$$


Considere as expansões

$$
I_{\nu}(\nu z)=\frac{\mathrm{e}^{\nu \sqrt{1+z^{2}}} \mathrm{e}^{\nu \log \frac{z}{1+\sqrt{1+z^{2}}}}}{\sqrt{2 \pi \nu}\left(1+z^{2}\right)^{\frac{1}{4}}}\left(1+\sum_{j=1}^{2 p-1} \frac{U_{j}(z)}{\nu^{j}}+O\left(\frac{1}{\nu^{2 p}}\right)\right),
$$

onde

$$
\begin{aligned}
& U_{0}(w)=1 \\
& U_{j}(w)=\frac{1}{2} w^{2}\left(1-w^{2}\right) \frac{d}{d w} U_{j-1}(w)+\frac{1}{8} \int_{0}^{w}\left(1-5 w^{2}\right) U_{j-1}(t) d t
\end{aligned}
$$

$\operatorname{com} w=\frac{1}{\sqrt{1+z^{2}}}, \mathrm{e}$

$$
I_{\nu}^{\prime}(\nu z)=\frac{\left(1+z^{2}\right)^{\frac{1}{4}} \mathrm{e}^{\nu \sqrt{1+z^{2}}} \mathrm{e}^{\nu \log \frac{z}{1+\sqrt{1+z^{2}}}}}{\sqrt{2 \pi \nu} z}\left(1+\sum_{j=1}^{2 p-1} \frac{V_{j}(z)}{\nu^{j}}+O\left(\frac{1}{\nu^{2 p}}\right)\right)
$$

com

$$
\begin{aligned}
& V_{0}(w)=1 \\
& V_{j}(w)=U_{j}(w)-\frac{w}{2}\left(1-w^{2}\right) U_{j-1}(w)-w^{2}\left(1-w^{2}\right) \frac{d}{d w} U_{j-1}(w) .
\end{aligned}
$$

Então

$$
\begin{aligned}
\log I_{\nu}(\nu z) & =\nu \sqrt{1+z^{2}}+\nu \log z-\nu \log \left(1+\sqrt{1+z^{2}}\right) \\
& -\frac{1}{2} \log 2 \pi \nu-\frac{1}{4} \log \left(1+z^{2}\right)+\log \left(1+\sum_{j=1}^{2 p-1} \frac{U_{j}(z)}{\nu^{j}}+O\left(\frac{1}{\nu^{2 p}}\right)\right) \\
\log I_{\nu}^{\prime}(\nu z) & =\nu \sqrt{1+z^{2}}+\nu \log z-\nu \log \left(1+\sqrt{1+z^{2}}\right)-\log z \\
& -\frac{1}{2} \log 2 \pi \nu+\frac{1}{4} \log \left(1+z^{2}\right)+\log \left(1+\sum_{j=1}^{2 p-1} \frac{V_{j}(z)}{\nu^{j}}+O\left(\frac{1}{\nu^{2 p}}\right)\right) .
\end{aligned}
$$

Substituindo $\nu$ por $\mu_{p-1, n}$ e $z$ por $\sqrt{-\lambda}$ em (6.3) temos

$$
\begin{aligned}
& \log \Gamma\left(-\lambda, S_{p-1, n} /\left(\mu_{p-1, n}\right)^{2}\right)-\log \Gamma\left(-\lambda, \dot{S}_{p-1, n} /\left(\mu_{p-1, n}\right)^{2}\right)=\frac{1}{2} \log (1-\lambda) \\
& \quad-\log \left(1+\sum_{j=1}^{2 p-1} \frac{U_{j}(\lambda)}{\mu_{p-1, n}^{j}}+O\left(\frac{1}{\mu_{p-1, n}^{2 p}}\right)\right)+\log \left(1+\sum_{j=1}^{2 p-1} \frac{V_{j}(\lambda)}{\mu_{p-1, n}^{j}}+O\left(\frac{1}{\mu_{p-1, n}^{2 p}}\right)\right) .
\end{aligned}
$$

Usando a expansão do logaritmo

$$
\log \left(1+\sum_{j=1}^{\infty} \frac{a_{j}}{z^{j}}\right)=\sum_{j=1}^{\infty} \frac{l_{j}}{z^{j}}
$$

onde $a_{0}=1, a_{1}=l_{1} \mathrm{e}$

$$
l_{j}=a_{j}-\sum_{k=1}^{j-1} \frac{j-k}{j} a_{k} l_{j-k}
$$


segue-se que,

$$
\begin{aligned}
& \log \Gamma\left(-\lambda, S_{p-1, n} /\left(\mu_{p-1, n}\right)^{2}\right)-\log \Gamma\left(-\lambda, \dot{S}_{p-1, n} /\left(\mu_{p-1, n}\right)^{2}\right)=\frac{1}{2} \log (1-\lambda) \\
& +\sum_{j=1}^{2 p-1} \frac{1}{\mu_{p-1, n}^{j}}\left(V_{j}(\lambda)-U_{j}(\lambda)+\sum_{k=1}^{j-1} \frac{j-k}{j}\left(V_{k}(\lambda) l_{j-k}^{\prime}(\lambda)-U_{k} l_{j-k}(\lambda)\right)\right)+O\left(\frac{1}{\mu_{p-1, n}^{2 p}}\right)
\end{aligned}
$$

onde $l_{j}^{\prime}(\lambda)$ é o termo da expansão do logaritmo com soma em $V_{j}(\lambda)$ e $l_{j}(\lambda)$ é o termo da expansão do logaritmo com soma em $U_{j}(\lambda)$. Definindo

$$
\begin{aligned}
\phi_{p-1, j}(\lambda) & =l_{j}^{\prime}(\lambda)-l_{j}(\lambda) \\
& =V_{j}(\lambda)-U_{j}(\lambda)+\sum_{k=1}^{j-1} \frac{j-k}{j}\left(V_{k}(\lambda) l_{j-k}^{\prime}(\lambda)-U_{k} l_{j-k}(\lambda)\right)
\end{aligned}
$$

obtemos

$$
\begin{aligned}
\log \Gamma\left(-\lambda, S_{p-1, n} /\left(\mu_{p-1, n}\right)^{2}\right)-\log \Gamma\left(-\lambda, \dot{S}_{p-1, n} /\left(\mu_{p-1, n}\right)^{2}\right)= \\
=\frac{1}{2} \log (1-\lambda)+\sum_{j=1}^{2 p-1} \phi_{p-1, j}(\lambda) \frac{1}{\mu_{p-1, n}^{j}}+O\left(\frac{1}{\mu_{p-1, n}^{2 p}}\right),
\end{aligned}
$$

e o resultado segue.

Proposição 12. As seqüências duplas $S_{p-1}$ e $\dot{S}_{p-1}$ possuem expoentes relativos $\left(p, \frac{2 p-1}{2}, \frac{1}{2}\right)$, genus relativos $\left(p,\left[\frac{2 p-1}{2}\right], 0\right)$, onde o colchete significa a parte inteira de $\frac{2 p-1}{2}$, e são espectralmente decompostas sobre $U_{p-1}$ com potência $\kappa=2$, comprimento $\ell=2 p$ e dominio $D_{\theta_{1}, c_{1}}$.

Demonstração. Estudaremos primeiro os expoentes finitos e os genus de $S_{p-1}$. Antes, lembraremos algumas relações. Uma das relações clássicas dos zeros das funções de Bessel é a seguinte: se $j_{\mu, k}$ é um zero da função $J_{\mu}(z)$ então

$$
k \pi+\frac{1}{2} \mu \pi+\frac{1}{4} \pi \leq j_{\mu, k} \leq(k+1) \pi+\frac{1}{2} \mu \pi+\frac{1}{4} \pi .
$$

Com esta relação, para $k$ suficientemente grande tem-se $j_{\mu, k} \sim k \pi$, e para $\mu$ suficientemente grande tem-se $j_{\nu, k} \sim \frac{1}{2} \mu \pi$. Lembrado que, para $n$ suficientemente grande $\lambda_{n} \sim n^{\frac{2}{2 p-1}}$, temos que $s_{1}=\frac{2 p-1}{2}$ e $s_{2}=\frac{1}{2}$. Para determinar $s_{0}$ é um pouco mais complicado, para isso temos

$$
\begin{aligned}
\sum_{n=1}^{t} \sum_{k=1}^{s} \frac{1}{j_{\mu_{p-1, n}, k}^{2 \alpha}} & \leq \sum_{n=1}^{t} \sum_{k=1}^{s} \frac{1}{\left(\pi k+\frac{\pi}{2}\left(\lambda_{p-1, n}\right)^{\frac{1}{2}}+\frac{\pi}{4}\right)^{2 \alpha}} \\
& \leq \sum_{n=1}^{t} \sum_{k=1}^{s} \frac{1}{\left(k+\left(\lambda_{p-1, n}\right)^{\frac{1}{2}}\right)^{2 \alpha}} \\
& \leq \sum_{n=1}^{t} \sum_{k=1}^{s} \frac{1}{\left(k+\left(n^{\frac{2}{2 p-1}}\right)^{\frac{1}{2}}\right)^{2 \alpha}}
\end{aligned}
$$


Usando a desigualdade de Young temos que $n^{\frac{1}{2 p-1}}+k \geq(n k)^{\frac{1}{2 p}}$. Logo,

$$
\sum_{n=1}^{t} \sum_{k=1}^{s} \frac{1}{\left(k+n^{\frac{1}{2 p-1}}\right)^{2 \alpha}} \leq \sum_{n=1}^{t} \sum_{k=1}^{s} \frac{1}{(n k)^{\frac{\alpha}{p}}}
$$

Dessa forma, a série $S_{p-1}$ converge se $\alpha>p$. Precisamos garantir a limitação inferior, para isso usamos a desigualdade

$$
\sum_{n=1}^{t} \sum_{k=1}^{s} \frac{1}{\left(\pi(k+1)+\frac{\pi}{2}\left(\lambda_{p-1, n}\right)^{\frac{1}{2}}+\frac{\pi}{4}\right)^{2 \alpha}} \leq \sum_{n=1}^{t} \sum_{k=1}^{s} \frac{1}{j_{\mu_{p-1, n}, k}^{2 \alpha}} .
$$

Utilizando o Teorema de Plana [49, temos

$$
\begin{aligned}
\sum_{n=1}^{t} \sum_{k=1}^{s} \frac{1}{\left(\pi(k+1)+\frac{\pi}{2} n^{\frac{1}{2 p-1}}+\frac{\pi}{4}\right)^{2 \alpha}} & \geq \sum_{n=1}^{t} \int_{1}^{s}\left(\pi x+\frac{\pi}{2}\left(\lambda_{p-1, n}\right)^{\frac{1}{2}}+\frac{\pi}{4}\right)^{-2 \alpha} d x \\
& \geq \sum_{n=1}^{t} \frac{1}{2 \alpha-1}\left(\frac{5 \pi}{4}+\frac{\pi}{2} n^{\frac{1}{2 p-1}}\right)^{-2 \alpha+1}
\end{aligned}
$$

Fazendo $t$ tender ao infinito, vemos que a série

$$
\sum_{n=1}^{\infty} \frac{1}{2 \alpha-1}\left(\frac{5 \pi}{4}+\frac{\pi}{2} n^{\frac{1}{2 p-1}}\right)^{-2 \alpha+1}
$$

converge se, e somente se, $\frac{2 \alpha-1}{2 p-1}>1$, e este único fato ocorre se, e somente se, $\alpha>p$. Desta forma $s_{0}=p$. Note que $\alpha>\frac{1}{2}$, como $s_{2}=\frac{1}{2}$.

Observamos que os expoentes e os genus para $\dot{S}$ são os mesmos, já que os zeros da derivada de uma função de Bessel são intercalados com os zeros da função de Bessel, ou seja, $j_{\mu, k}<$ $j_{\mu, k}^{\prime}<j_{\mu, k+1}$.

O restante da proposição segue diretamente dos lemas 12 e 14. E ainda, o comprimento $\ell$ da decomposição é precisamente $2 p$, pois e $\left(U_{p-1}\right)=2 p-1$ e, portanto, o maior inteiro $h$ tal que $h-1=\sigma_{h} \leq 2 p-1$ é $2 p$. Isto completa a prova.

Observação 1. Pelo teorema 4, apenas os termos com $\sigma_{h}=1, \sigma_{h}=3, \ldots, \sigma_{h}=2 p-1$, com $h=2,4, \ldots, 2 p$, respectivamente, aparecem na fórmula do teorema 5 , pelo lema 13 .

Agora faremos um estudo das funções $\phi_{p-1, j}(\lambda)$ que aparecem no lema 15.

Lema 16. Para todo índice $j \in \mathbb{N}$, as funçôes $\phi_{p-1, j}(\lambda)$ são polinômios em $w=\frac{1}{\sqrt{1-\lambda}} d a$ forma

$$
\phi_{p-1, j}(\lambda)=\sum_{k=0}^{j} a_{p-1, j, k} w^{2 k+j},
$$

e ainda $\phi_{p-1, j}(0)=0$ para todo $j \geq 1$. 
Demonstração. A prova que as funções $\phi_{p-1, j}(\lambda)$ são polinômios em $w=\frac{1}{\sqrt{1-\lambda}}$ e possuem a representação acima é aplicação direta da definição das $\phi_{p-1, j}(\lambda)$, que está na equação (6.4). Faremos apenas a prova da segunda afirmação, ou seja, que $\phi_{p-1, j}(0)=0$ para todo $j$. Usaremos indução. Para $j=1$ temos

$$
\begin{aligned}
\phi_{p-1,1}(\lambda) & =l_{1}^{\prime}(\lambda)-l_{1}(\lambda)=V_{1}(\lambda)-U_{1}(\lambda) \\
& =-\frac{1}{2} \frac{1}{(1-\lambda)^{\frac{1}{2}}}+\frac{1}{2} \frac{1}{(1-\lambda)^{\frac{3}{2}}}
\end{aligned}
$$

e assim $\phi_{p-1,1}(0)=0$. Suponha que $\phi_{p-1, k}(0)=0$ para $k=1, \ldots, j-1$ e provaremos para $k=j$. Considerando $\phi_{p-1, j}(\lambda)$ como função de $w=(1-\lambda)^{-\frac{1}{2}}$, temos que, quando $\lambda=0, w$ é igual a 1. Então

$$
\begin{aligned}
\phi_{p-1, j}(w) & =l_{j}^{\prime}(w)-l_{j}(w) \\
= & V_{j}(w)-U_{j}(w)+\sum_{s=1}^{j-1} \frac{j-s}{j}\left(U_{s}(w) l_{j-s}(w)-V_{s}(w) l_{j-s}^{\prime}(w)\right) \\
= & V_{j}(w)-U_{j}(w)+\sum_{s=1}^{j-1} \frac{j-s}{j}\left(U_{s}(w)\left(l_{j-s}(w)-l_{j-s}^{\prime}(w)\right)\right) \\
& +\sum_{s=1}^{j-1}\left(1-w^{2}\right)\left(\frac{w}{2} U_{s-1}(w)+w^{2} \frac{d}{d w} U_{s-1}(w)\right) l_{j-s}^{\prime}(w) .
\end{aligned}
$$

E usando a hipótese de indução temos que $\phi_{p-1, j}(1)=0$, como função de $w$. Logo $\phi_{p-1, j}(0)=$ 0 , como função de $\lambda$, o que termina prova.

Por definição, na equação (1.19),

$$
\Phi_{p-1, j}(s)=\int_{0}^{\infty} t^{s-1} \frac{1}{2 \pi \mathrm{i}} \int_{\Lambda_{\theta, c}} \frac{\mathrm{e}^{-\lambda t}}{-\lambda} \phi_{p-1, j}(\lambda) d \lambda d t
$$

então:

Corolário 4. Para todo $j \geq 1$, a expansão de Laurent das funçôes $\Phi_{p-1,2 j+1}(s)$ em $s=0$ possuem os coeficientes:

$$
\underset{s=0}{\operatorname{Res}_{0}} \Phi_{p-1,2 j+1}(s)=2 \sum_{k=1}^{2 j+1} a_{p-1,2 j+1, k} \sum_{t=2}^{k+j} \frac{1}{2 t-1}, \quad \operatorname{Res}_{s=0} \Phi_{p-1,2 j+1}(s)=0,
$$

em particular, quando $j=0$ temos

$$
\operatorname{Res}_{s=0} \Phi_{p-1,1}(s)=2 a_{p-1,1,1}=1, \quad \operatorname{Res}_{s=0} \Phi_{p-1,1}(s)=0 .
$$

Demonstração. Usando a definição das $\Phi_{p-1,2 j+1}(s)$ e calculando a integral por meio da equação (1.23) temos que

$$
\Phi_{p-1,2 j+1}(s)=\sum_{k=0}^{2 j+1} a_{p-1,2 j+1, k} \frac{\Gamma\left(s+\frac{2(k+j)+1}{2}\right)}{s \Gamma\left(\frac{2(k+j)+1}{2}\right)} .
$$


Lembrando que

$$
\operatorname{Res}_{s=0} \frac{\Gamma\left(s+\frac{2 j+1}{2}\right)}{\Gamma\left(\frac{2 j+1}{2}\right) s}=-\gamma-2 \log 2+2 \sum_{k=1}^{j} \frac{1}{2 k-1} \quad \text { e } \quad \operatorname{Res}_{s=0} \frac{\Gamma\left(s+\frac{2 j+1}{2}\right)}{\Gamma\left(\frac{2 j-1}{2}\right) s}=1,
$$

obtemos

$$
\begin{aligned}
& \underset{\operatorname{Res}_{0}}{s=0} \Phi_{p-1,2 j+1}(s)=\sum_{k=0}^{2 j+1} a_{p-1,2 j+1, k}\left(-\gamma-2 \log 2+2 \sum_{t=1}^{k+j} \frac{1}{2 t-1}\right) \\
& \operatorname{Res}_{s=0} \Phi_{p-1,2 j+1}(s)=\sum_{k=0}^{2 j+1} a_{p-1,2 j+1, k} .
\end{aligned}
$$

Assim, usando o lema anterior temos o resultado. Em particular,

$$
\operatorname{Res}_{s=0} \Phi_{p-1,1}(s)=2 a_{p-1,1,1}, \quad \operatorname{Res}_{s=0} \Phi_{p-1,1}(s)=0 .
$$

Como $a_{p-1,1,1}=\frac{1}{2}$,

$$
\operatorname{Res}_{s=0} \Phi_{p-1,1}(s)=1
$$

Nosso próximo passo é determinar os termos $A_{0,0}(0)$ e $A_{0,1}^{\prime}(0)$ definidos na equação (1.21). Os termos relativos à seqüência $\dot{S}_{p-1}$ serão representados por um ponto em cima. Temos o seguinte resultado

\section{Lema 17.}

$$
\begin{aligned}
& \mathcal{A}_{p-1,0,0}=A_{p-1,0,0}(s)-\dot{A}_{p-1,0,0}(s)=0 \\
& \mathcal{A}_{p-1,0,1,}=A_{p-1,0,1}(s)-\dot{A}_{p-1,0,1}(s)=\frac{1}{2} \zeta\left(2 s, U_{p-1}\right) .
\end{aligned}
$$

Demonstração. A definição da equação (1.21) no caso de $S_{p-1}$ fica,

$$
\begin{aligned}
& A_{p-1,0,0}(s)=\sum_{n=1}^{\infty}\left(a_{p-1,0,0, n}-\sum_{j=1}^{p} b_{p-1,2 j-1,0,0} \mu_{p-1, n}^{-2 j+1}\right) \mu_{p-1, n}^{-2 s}, \\
& A_{p-1,0,1}(s)=\sum_{n=1}^{\infty}\left(a_{p-1,0,1, n}-\sum_{j=1}^{p} b_{p-1,2 j-1,0,1} \mu_{p-1, n}^{-2 j+1}\right) \mu_{p-1, n}^{-2 s} .
\end{aligned}
$$

No caso de $\dot{S}_{p-1}$ temos,

$$
\begin{aligned}
& \dot{A}_{p-1,0,0}(s)=\sum_{n=1}^{\infty}\left(\dot{a}_{p-1,0,0, n}-\sum_{j=1}^{p} \dot{b}_{p-1,2 j-1,0,0} \mu_{p-1, n}^{-2 j+1}\right) \mu_{p-1, n}^{-2 s}, \\
& \dot{A}_{p-1,0,1}(s)=\sum_{n=1}^{\infty}\left(\dot{a}_{p-1,0,1, n}-\sum_{j=1}^{p} \dot{b}_{p-1,2 j-1,0,1} \mu_{p-1, n}^{-2 j+1}\right) \mu_{p-1, n}^{-2 s} .
\end{aligned}
$$


Assim precisamos da expansão para $\lambda$ grande das funções $l_{2 j-1}(\lambda), l_{2 j-1}^{\prime}(\lambda)$, para $j=$ $1,2, \ldots, p, \log \Gamma\left(-\lambda, S_{p-1, n} / \mu_{p-1, n}^{2}\right)$ e $\log \Gamma\left(-\lambda, \dot{S}_{p-1, n} / \mu_{p-1, n}^{2}\right)$. Usando as expansões que estão na equação (4.29), obtemos

$$
\begin{aligned}
\log \Gamma\left(-\lambda, S_{p-1, n} / \mu_{p-1, n}^{2}\right) & =\frac{1}{2} \log 2 \pi+\left(\mu_{p-1, n}+\frac{1}{2}\right) \log \mu_{p-1, n}-\mu_{p-1, n} \log 2 \\
& -\log \Gamma\left(\mu_{p-1, n}+1\right)+\frac{1}{2}\left(\mu_{p-1, n}+\frac{1}{2}\right) \log (-\lambda)+O\left(\mathrm{e}^{-\mu_{p-1, n} \sqrt{-\lambda}}\right) \\
\log \Gamma\left(-\lambda, \dot{S}_{p-1, n} / \mu_{p-1, n}^{2}\right) & =\frac{1}{2} \log 2 \pi+\left(\mu_{p-1, n}+\frac{1}{2}\right) \log \mu_{p-1, n}-\mu_{p-1, n} \log 2 \\
& -\log \Gamma\left(\mu_{p-1, n}+1\right)+\frac{1}{2}\left(\mu_{p-1, n}-\frac{1}{2}\right) \log (-\lambda)+O\left(\mathrm{e}^{-\mu_{p-1, n} \sqrt{-\lambda}}\right) .
\end{aligned}
$$

E então temos os valores

$$
\begin{aligned}
& a_{p-1,0,0, n}=\frac{1}{2} \log 2 \pi+\left(\mu_{p-1, n}+\frac{1}{2}\right) \log \mu_{p-1, n}-\mu_{p-1, n} \log 2-\log \Gamma\left(\mu_{p-1, n}+1\right), \\
& a_{p-1,0,1, n}=\frac{1}{2}\left(\mu_{p-1, n}+\frac{1}{2}\right), \\
& \dot{a}_{p-1,0,0, n}=\frac{1}{2} \log 2 \pi+\left(\mu_{p-1, n}+\frac{1}{2}\right) \log \mu_{p-1, n}-\mu_{p-1, n} \log 2-\log \Gamma\left(\mu_{p-1, n}+1\right), \\
& \dot{a}_{p-1,0,1, n}=\frac{1}{2}\left(\mu_{p-1, n}-\frac{1}{2}\right),
\end{aligned}
$$

Note que $b_{p-1,2 j-1,0,0}=b_{p-1,2 j-1,0,1}=0$ e $\dot{b}_{p-1,2 j-1,0,0}=\dot{b}_{p-1,2 j-1,0,1}=0$, como não temos termos constantes para $j=1,2, \ldots p$ nas expansões de $l_{2 j-1}(\lambda)$ e $l_{2 j-1}^{\prime}(\lambda)$. Então

$$
\begin{aligned}
& \mathcal{A}_{p-1,0,0}=A_{p-1,0,0}(s)-\dot{A}_{p-1,0,0}(s)=0 \\
& \mathcal{A}_{p-1,0,1,}=A_{p-1,0,1}(s)-\dot{A}_{p-1,0,1}(s)=\frac{1}{2} \sum_{n=1}^{\infty} m_{p-1, n} \mu_{p-1, n}^{-2 s}=\frac{1}{2} \zeta\left(2 s, U_{p-1}\right),
\end{aligned}
$$

e o resultado segue.

Com todos os lemas anteriores e respectivos corolários, podemos usar o teorema 5 e o seu corolário para obtermos o resultado a seguir.

Proposição 13. As funçôes $t_{p-1}(s)$ e $t_{p-1}^{\prime}(s)$ em $s=0$ assumem os valores

$$
\begin{aligned}
& t_{p-1}(0)=-\frac{1}{2} \zeta\left(0, U_{p-1}\right), \\
& t_{p-1}^{\prime}(0)=-\zeta^{\prime}\left(0, U_{p-1}\right)+\sum_{j=0}^{p-1} \operatorname{Res}_{s=0} \Phi_{p-1,2 j+1}(s) \operatorname{Res}_{s=2 j+1} \zeta\left(s, U_{p-1}\right) .
\end{aligned}
$$

Demonstração. Seguindo a idéia acima e usando o corolário 4 e os lemas 13 e 17 o resultado segue. 
Dividiremos $t_{p-1}(0)$ e $t_{p-1}^{\prime}(0)$ da seguinte maneira

$$
\begin{aligned}
& t_{p-1}(0)=t_{p-1, \mathrm{reg}}(0)+t_{p-1, \mathrm{sing}}(0), \\
& t_{p-1}^{\prime}(0)=t_{p-1, \mathrm{reg}}^{\prime}(0)+t_{p-1, \mathrm{sing}}^{\prime}(0),
\end{aligned}
$$

onde,

$$
\begin{aligned}
t_{p-1, \mathrm{reg}}(0) & =-\frac{1}{2} \zeta\left(0, U_{p-1}\right), \\
t_{p-1, \mathrm{sing}}(0) & =0 \\
t_{p-1, \mathrm{reg}}^{\prime}(0) & =-\zeta^{\prime}\left(0, U_{p-1}\right), \\
t_{p-1, \mathrm{sing}}^{\prime}(0) & =\frac{1}{2} \sum_{j=0}^{p-1} \operatorname{Res}_{s=0} \Phi_{p-1,2 j+1}(s) \operatorname{Res}_{s=2 j+1} \zeta\left(s, U_{p-1}\right),
\end{aligned}
$$

e chamaremos de partes regulares os termos $t_{p-1, \mathrm{reg}}(0)$ e $t_{p-1, \mathrm{reg}}^{\prime}(0)$, e de partes singulares os termos $t_{p-1, \operatorname{sing}}(0)$ e $t_{p-1, \text { sing }}^{\prime}(0)$.

\subsection{Estudo de $t_{q}(s)$}

O estudo destas funções é uma generalização natural do caso anterior, mas como os detalhes são sutis faremos a maioria das provas. Nosso objetivo é usar o teorema 15, então, consideraremos as seqüências duplas $S_{q}=\left\{m_{q, n}: j_{\mu_{q, n}, k}^{2}\right\}_{n=1}^{\infty}$ e $S_{q, \pm}=\left\{m_{q, n}: j_{\mu_{q, n} \pm \alpha_{q}, k}^{2}\right\}_{n=1}^{\infty}$, já que $Z_{q}(s)=\zeta\left(s, S_{q}\right), Z_{q, \pm}(s)=\zeta\left(s, S_{q, \pm}\right)$, onde $q=0,1, \ldots, p-2$ e $\alpha_{q}=-p+q+1$. Note que a análise de $S_{q}$ é similar à análise de $S_{p-1}$, por isso nos concentraremos no estudo das outras duas seqüências. Verificaremos primeiro que $S_{q}$ e $S_{q, \pm}$ são espectralmente decompostas sobre $U_{q}=\left\{m_{q, n}: \mu_{q, n}\right\}_{n=1}^{\infty}$, ou seja, satisfazem a definição 13 ,

Lema 18. A seqüência $U_{q}$ é uma seqüência totalmente regular do tipo espectral com ordem infinita, $\mathrm{e}\left(U_{q}\right)=\mathrm{g}\left(U_{q}\right)=2 p-1, e$

$$
\zeta\left(s, U_{q}\right)=\sum_{t=0}^{\infty}\left(\begin{array}{c}
-\frac{s}{2} \\
t
\end{array}\right) \zeta\left(\frac{s}{2}+t, \tilde{U}_{q}\right) \alpha_{q}^{t},
$$

onde, $\tilde{U}_{q}=\left\{m_{q, n}: \lambda_{q, n}\right\}_{n=1}^{\infty}$, e $\lambda_{q, n}$ é um autovalor de uma autoforma coexata de $N$ de dimensão $q$, e $q=0,1, \ldots, p-2$. Além disso, $\zeta\left(s, U_{q}\right)$ possui polos simples em $s=2(p-k)-1$ para $k=0,1,2, \ldots$

Demonstração. As provas que $\mathrm{e}\left(U_{q}\right)=\mathrm{g}\left(U_{q}\right)=2 p-1, \zeta\left(s, U_{q}\right)$ possui polos simples em $s=2(p-k)-1$ para $k=0,1,2, \ldots$ e que $U_{q}$ é uma seqüência totalmente regular do tipo espectral com ordem infinita seguem com argumentos análogos aos da prova do lema 13, Para a representação de $\zeta\left(s, U_{q}\right)$ temos,

$$
\zeta\left(s, U_{q}\right)=\sum_{n=1}^{\infty} m_{q, n}\left(\lambda_{q, n}+\alpha_{q}^{2}\right)^{-\frac{s}{2}}=\sum_{t=0}^{\infty}\left(\begin{array}{c}
-\frac{s}{2} \\
t
\end{array}\right) \zeta\left(\frac{s}{2}+t, \tilde{U}_{q}\right) \alpha_{q}^{2 t} .
$$


Começamos agora a análise das seqüências duplas $S_{q, \pm}$. Como os elementos de $S_{q, \pm}$ não são múltiplos de zeros de funções de Bessel (e nem da derivada de uma função de Bessel), teremos um pouco mais de trabalho. Mas isto não é um problema muito grande, já que tais elementos são zeros de combinações lineares de funções de Bessel e de suas derivadas, e isto torna possível a análise a seguir.

Para $c \in \mathbb{R}$, defina as funções

$$
\hat{J}_{\nu, c}(z)=c J_{\nu}(z)+z J_{\nu}^{\prime}(z)
$$

Da definição em série da função de Bessel na equação (4.33), obtemos, próximo a $z=0$,

$$
\hat{J}_{\nu, c}(z)=\left(1+\frac{c}{\nu}\right) \frac{z^{\nu}}{2^{\nu} \Gamma(\nu)}
$$

Isto significa que a função $z^{-\nu} \hat{J}_{\nu, c}(z)$ é uma função par em $z$. Considere $\hat{j}_{\nu, c, k}$ os zeros de $\hat{J}_{\nu, c}(z)$ em ordem crescente. Pelo teorema da fatorização de Hadamard [12], tem-se

$$
z^{-\nu} \hat{J}_{\nu(z), c}=z^{-\nu} \hat{J}_{\nu, c}(z) \prod_{k=-\infty}^{+\infty}\left(1-\frac{z}{\hat{j}_{\nu, c, k}}\right)
$$

e portanto

$$
\hat{J}_{\nu, c}(z)=\left(1+\frac{c}{\nu}\right) \frac{z^{\nu}}{2^{\nu} \Gamma(\nu)} \prod_{k=1}^{\infty}\left(1-\frac{z^{2}}{\hat{j}_{\nu, c, k}^{2}}\right) .
$$

Usando a equação (4.34), quando $-\pi<\arg (z)<\frac{\pi}{2}$, obtemos

$$
\hat{J}_{\nu, c}(\mathrm{i} z)=\mathrm{e}^{\frac{\pi}{2} \mathrm{i} \nu}\left(c I_{\nu}(z)+z I_{\nu}^{\prime}(z)\right) .
$$

Então definimos, quando $-\pi<\arg (z)<\frac{\pi}{2}$

$$
\hat{I}_{\nu, c}(z)=\mathrm{e}^{-\frac{\pi}{2} \mathrm{i} \nu} T_{\nu, c}(\mathrm{i} z)
$$

e assim

$$
\hat{I}_{\nu, \pm \alpha_{q}}(z)= \pm \alpha_{q} I_{\nu}(z)+z I_{\nu}^{\prime}(z)=\left(1 \pm \frac{\alpha_{q}}{\nu}\right) \frac{z^{\nu}}{2^{\nu} \Gamma(\nu)} \prod_{k=1}^{\infty}\left(1+\frac{z^{2}}{\hat{j}_{\nu, \pm \alpha_{q}, k}^{2}}\right)
$$

Usando a definição da função Gamma (1.17) provaremos o seguinte:

Lema 19. O logaritmo da função Gamma associada a seqüência $S_{q, \pm, n} / \mu_{q, n}^{2}$ é da forma

$$
\begin{aligned}
\log \Gamma\left(-\lambda, S_{q, \pm, n} / \mu_{q, n}^{2}\right)= & -\log \prod_{k=1}^{\infty}\left(1+\frac{(-\lambda) \mu_{q, n}^{2}}{\hat{j}_{\mu_{q, n}, \pm \alpha_{q}, k}^{2}}\right) \\
= & -\log \hat{I}_{\mu_{q, n}, \pm \alpha_{q}}\left(\mu_{q, n} \sqrt{-\lambda}\right)+\mu_{q, n} \log \sqrt{-\lambda}+\mu_{q, n} \log \mu_{q, n} \\
& -\mu_{q, n} \log 2-\log \Gamma\left(\mu_{q, n}\right)+\log \left(1 \pm \frac{\alpha_{q}}{\mu_{q, n}}\right) .
\end{aligned}
$$


Demonstração. Basta usar a representação que está na equação (6.8) e obtemos a fórmula do enunciado.

Lema 20. A expansão assintótica de

$$
2 \log \Gamma\left(-\lambda, S_{q, n} / \mu_{q, n}^{2}\right)-\log \Gamma\left(-\lambda, S_{q,+, n} / \mu_{q, n}^{2}\right)-\log \Gamma\left(-\lambda, S_{q,-, n} / \mu_{q, n}^{2}\right),
$$

para $n$ suficientemente grande, uniformemente em $\lambda \in D_{\theta_{1}, c_{2}}$, é da seguinte forma:

$$
\begin{aligned}
& 2 \log \Gamma\left(-\lambda, S_{q, n} / \mu_{q, n}^{2}\right)-\log \Gamma\left(-\lambda, S_{q,+, n} / \mu_{q, n}^{2}\right)-\log \Gamma\left(-\lambda, S_{q,-, n} / \mu_{q, n}^{2}\right)= \\
& \quad=\log (1-\lambda)+\sum_{j=1}^{2 p-1} \phi_{q, j}(\lambda) \frac{1}{\mu_{q, n}^{j}}+O\left(\frac{1}{\mu_{q, n}^{2 p}}\right) .
\end{aligned}
$$

Demonstração. As seqüências $S_{q, n}$ e $S_{q, n, \pm}$ são seqüências de números reais, logo podemos considerar o mesmo $\theta_{1}$ da seqüência $S_{p-1, n}$ e $c_{2}=\frac{1}{2} \min \left(j_{\mu_{q, 0}}^{2}, j_{\mu_{q, 0}, \pm \alpha_{q}}^{2}\right)$, para $D_{\theta_{1}, c_{2}}$, o domínio de $\lambda$.

Agora, considerando as representações dadas nos lemas 14 e 19 para $\log \Gamma\left(-\lambda, S_{q, n} / \mu_{q, n}^{2}\right)$, $\log \Gamma\left(-\lambda, S_{q,+, n} / \mu_{q, n}^{2}\right)$ e $\log \Gamma\left(-\lambda, S_{q,-, n} / \mu_{q, n}^{2}\right)$, temos

$$
\begin{aligned}
2 \log \Gamma\left(-\lambda, S_{q, n} / \mu_{q, n}^{2}\right)-\log \Gamma\left(-\lambda, S_{q,+, n} / \mu_{q, n}^{2}\right)-\log \Gamma\left(-\lambda, S_{q,-, n} / \mu_{q, n}^{2}\right)= \\
=-2 \log I_{\mu_{q, n}}\left(\mu_{q, n} \sqrt{-\lambda}\right)+\log \hat{I}_{\mu_{q, n}, \alpha_{q}}\left(\mu_{q, n} \sqrt{-\lambda}\right)+\log \hat{I}_{\mu_{q, n,-\alpha_{q}}}\left(\mu_{q, n} \sqrt{-\lambda}\right) \\
\quad-2 \log \mu_{q, n}-\log \left(1-\frac{\alpha_{q}^{2}}{\mu_{q, n}^{2}}\right) .
\end{aligned}
$$

Usando as expansões dadas no lema 15 para $I_{\nu}(\nu z)$ e $I_{\nu}^{\prime}(\nu z)$, obtemos a expansão para $\hat{I}_{\nu, \pm \alpha_{q}}(\nu z)$, da seguinte maneira,

$$
\begin{aligned}
\hat{I}_{\nu, \pm \alpha_{q}}(\nu z) & = \pm \alpha_{q} I_{\nu}(\nu z)+\nu z I_{\nu}^{\prime}(\nu z) \\
& =\sqrt{\nu}\left(1+z^{2}\right)^{\frac{1}{4}} \frac{\mathrm{e}^{\nu \sqrt{1+z^{2}}} \mathrm{e}^{\nu \log \frac{z}{1+\sqrt{1+z^{2}}}}}{\sqrt{2 \pi}}\left(1+\sum_{j=1}^{2 p-1} W_{ \pm \alpha_{q}, j}(z) \frac{1}{\nu^{j}}+O\left(\frac{1}{\nu^{2 p}}\right)\right),
\end{aligned}
$$

onde $W_{ \pm \alpha_{q}, j}(z)=V_{j}(z) \pm \frac{\alpha_{q}}{\sqrt{1+z^{2}}} U_{j}(z)$. Então

$$
\begin{aligned}
\log \hat{I}_{\nu, \pm \alpha_{q}}(\nu z) & =\nu \sqrt{1+z^{2}}+\nu \log z-\nu \log \left(1+\sqrt{1+z^{2}}\right)+\log \nu+\frac{1}{4} \log \left(1+z^{2}\right) \\
& -\frac{1}{2} \log 2 \pi \nu+\log \left(1+\sum_{j=1}^{2 p-1} W_{ \pm \alpha_{q}, j}(z) \frac{1}{\nu^{j}}+O\left(\frac{1}{\nu^{2 p}}\right)\right) .
\end{aligned}
$$

Substituindo $\nu$ por $\mu_{q, n}$ e $z$ por $\sqrt{-\lambda}$ obtemos

$$
\begin{aligned}
& 2 \log \Gamma\left(-\lambda, S_{q, n} / \mu_{q, n}^{2}\right)-\log \Gamma\left(-\lambda, S_{q,+, n} / \mu_{q, n}^{2}\right)-\log \Gamma\left(-\lambda, S_{q,-, n} / \mu_{q, n}^{2}\right)=\log (1-\lambda) \\
& -2 \log \left(1+\sum_{j=1}^{2 p-1} \frac{U_{j}(\lambda)}{\mu_{q, n}^{j}}+O\left(\frac{1}{\mu_{q, n}^{2 p}}\right)\right)+\log \left(1+\sum_{j=1}^{2 p-1} \frac{W_{+\alpha_{q}, j}}{\mu_{q, n}^{j}}+O\left(\frac{1}{\mu_{q, n}^{2 p}}\right)\right) \\
& +\log \left(1+\sum_{j=1}^{2 p-1} \frac{W_{-\alpha_{q}, j}}{\mu_{q, n}^{j}}+O\left(\frac{1}{\mu_{q, n}^{2 p}}\right)\right) .
\end{aligned}
$$


Agora, usando a expansão do logaritmo, que também está no lema 15, temos

$$
\begin{aligned}
2 \log \Gamma\left(-\lambda, S_{q, n} / \mu_{q, n}^{2}\right) & -\log \Gamma\left(-\lambda, S_{q,+, n} / \mu_{q, n}^{2}\right)-\log \Gamma\left(-\lambda, S_{q,-, n} / \mu_{q, n}^{2}\right)=\log (1-\lambda) \\
& +\sum_{j=1}^{p}\left(-2 l_{2 j-1}(\lambda)+l_{2 j-1}^{+}(\lambda)+l_{2 j-1}^{-}(\lambda)\right) \frac{1}{\mu_{q, n}^{2 j-1}} \\
& +\sum_{j=1}^{p-1}\left(-2 l_{2 j}(\lambda)+l_{2 j}^{+}(\lambda)+l_{2 j}^{-}(\lambda)+\frac{\alpha_{q}^{2 j}}{j}\right) \frac{1}{\mu_{q, n}^{2 j}}+O\left(\frac{1}{\mu_{q, n}^{2 p}}\right),
\end{aligned}
$$

onde $l_{j}(\lambda)$ é o termo da expansão do logaritmo com soma em $U_{j}(\lambda)$ e $l_{j}^{ \pm}(\lambda)$ é o termo da expansão do logaritmo com soma em $W_{ \pm \alpha_{q}, j}(\lambda)$. Definimos então

$$
\begin{aligned}
\phi_{q, 2 j-1}(\lambda) & =-2 l_{2 j-1}(\lambda)+l_{2 j-1}^{+}(\lambda)+l_{2 j-1}^{-}(\lambda) \\
\phi_{q, 2 j}(\lambda) & =-2 l_{2 j}(\lambda)+l_{2 j}^{+}(\lambda)+l_{2 j}^{-}(\lambda)+\frac{\alpha_{q}^{2 j}}{j} .
\end{aligned}
$$

Logo

$$
\begin{aligned}
& 2 \log \Gamma\left(-\lambda, S_{q, n} / \mu_{q, n}^{2}\right)-\log \Gamma\left(-\lambda, S_{q,+, n} / \mu_{q, n}^{2}\right)-\log \Gamma\left(-\lambda, S_{q,-, n} / \mu_{q, n}^{2}\right)= \\
& \quad=\log (1-\lambda)+\sum_{j=1}^{2 p-1} \phi_{q, j}(\lambda) \frac{1}{\mu_{q, n}^{j}}+O\left(\frac{1}{\mu_{q, n}^{2 p}}\right)
\end{aligned}
$$

e o resultado está provado.

Proposição 14. As seqüências duplas $S_{q, \pm}$ possuem expoentes relativos $\left(p, \frac{2 p-1}{2}, \frac{1}{2}\right)$, genus relativos $\left(p,\left[\frac{2 p-1}{2}\right], 0\right)$, e são espectralmente decompostas sobre $U_{q}$ com potência $\kappa=2$, comprimento $\ell=2 p$ e dominio $D_{\theta_{1}, c_{2}}$

Demonstração. A prova dos expoentes finitos é a mesma da proposição 12, Basta observar que os zeros de $J_{\mu}(z)$ e $\hat{J}_{\mu, c}(z)$ são intercalados(ver [48], 15.23), ou seja, $j_{\mu, k}<\hat{j}_{\mu, c, k}<j_{\mu, k+1}$.

A existência de uma expansão assintótica completa da função Gamma, $\log \Gamma\left(-\lambda, S_{q, \pm, n}\right)$ segue do lema 19, E claramente $S_{q, \pm}$ é uma seqüência totalmente regular do tipo espectral com ordem infinita. A existência da expansão uniforme dada no lema anterior garante que $S_{q, \pm}$ é espectralmente decomposta sobre $U_{q}$, com potência $\kappa=2$. O comprimento $\ell$ da decomposição é precisamente $2 p$. De fato, e $\left(U_{q}\right)=2 p-1$ e, portanto, o maior inteiro $h$ tal que $h-1=\sigma_{h} \leq 2 p-1$ é $2 p$.

Note que a observação 1 também é válida neste caso.

Vamos agora estudar as funções $\phi_{q, j}(\lambda)$ e as funções $\Phi_{q, j}(s)$ definidas pela equação (1.19). 
Lema 21. Para todo $j$ e todo $0 \leq q \leq p-2$, as funçôes $\phi_{q, j}(\lambda)$ são polinômios da forma abaixo, onde $w=\frac{1}{\sqrt{1-\lambda}}$

$$
\begin{aligned}
\phi_{q, 2 j-1}(\lambda) & =\sum_{k=0}^{2 j-1} a_{q, 2 j-1, k} w^{2 k+2 j-1} \\
\phi_{q, 2 j}(\lambda) & =\sum_{k=0}^{2 j} a_{q, 2 j, k} w^{2 k+2 j}+\frac{\alpha_{q}^{2 j}}{j} .
\end{aligned}
$$

Além disso, para todo $j$ e todo $0 \leq q \leq p-2$, tem-se $\phi_{q, j}(0)=0$.

Demonstração. A prova de que $\phi_{q, j}(\lambda)$, para $0 \leq q \leq p-2$, tem a representação acima é a aplicação direta da definição. Consideraremos todas as funções como funções de $w$. Provaremos que $\phi_{q, j}(1)=0$ para $0 \leq q \leq p-2$. Faremos a prova por indução em $j$. Considere as hipóteses de indução, para $1 \leq k \leq j-1$ :

$$
\begin{aligned}
\phi_{q, 2 k-1}(1) & =0 \\
\phi_{q, 2 k}(1) & =0 \\
l_{2 k-1}^{-}(1)-l_{2 k-1}^{+}(1) & =\frac{-2 \alpha_{q}^{2 k-1}}{2 k-1}, \\
l_{2 k}^{-}(1)-l_{2 k}^{+}(1) & =0
\end{aligned}
$$

onde as funções $\phi_{q, j}$ estão definidas em (6.9).

Para $k=1$ temos

$$
\begin{aligned}
\phi_{q, 1}(w) & =-2 l_{1}(w)+l_{1}^{+}(w)+l_{1}^{-}(w) \\
& =-2 U_{1}(w)+V_{1}(w)+\alpha_{q} U_{0}(w)+V_{1}(w)-\alpha_{q} U_{0}(w) \\
& =-w+w^{3},
\end{aligned}
$$

$\operatorname{assim} \phi_{i, j}(1)=0, \mathrm{e}$

$$
\begin{aligned}
\phi_{q, 2}(w) & =-2 l_{2}(w)+l_{2}^{+}(w)+l_{2}^{-}(w)+\alpha_{q}^{2} \\
& =-2 U_{2}(w)+2 V_{2}(w)+U_{1}(w)^{2}-V_{1}(w)^{2} \\
& =-\frac{3}{2} w^{2}+2 w^{4}-\frac{3}{2} w^{6}+1,
\end{aligned}
$$

então $\phi_{q, 2}(1)=0$. E para as equações $(\underline{6.12})$ e $(\underline{6.13})$ temos

$$
l_{1}^{-}(1)-l_{1}^{+}(1)=-2 \alpha_{q} \text { e } l_{2}^{-}(1)-l_{2}^{+}(1)=0 .
$$

Observamos que $U_{k}(1)=V_{k}(1)$ para todo $k$. Agora, para $k=j$ temos

$$
\begin{aligned}
& l_{2 j-1}^{-}(1)-l_{2 j-1}^{+}(1)=U_{2 j-1}(1)-\alpha_{q} U_{2 j-2}(1)-U_{2 j-1}(1)-\alpha_{q} U_{2 j-2}(1) \\
& -\sum_{k=1}^{2 j-2} \frac{2 j-1-k}{2 j-1}\left(U_{k}(1)\left(l_{2 j-1-k}^{-}(1)-l_{2 j-1-k}^{+}(1)\right)\right) \\
& +\sum_{k=1}^{2 j-2} \frac{2 j-1-k}{2 j-1}\left(\alpha_{q} U_{k-1}(1)\left(l_{2 j-1-k}^{-}(1)+l_{2 j-1-k}^{+}(1)\right)\right) .
\end{aligned}
$$


Usando as hipóteses de indução temos

$$
\begin{aligned}
& l_{2 j-1}^{-}(1)-l_{2 j-1}^{+}(1)=-2 \alpha_{q} U_{2 j-2}(1)+\sum_{k=1}^{j-1} \frac{2(j-k)}{2 j-1} \alpha_{q} U_{2 k-2}(1)\left(2 l_{2(j-k)}-\frac{\alpha_{q}^{2(j-k)}}{j-k}\right) \\
& -\sum_{k=1}^{j-1} \frac{2(j-k)-1}{2 j-1} U_{2 k}(1) \frac{-2 \alpha_{q}^{2(j-k)-1}}{2(j-k)-1}+\sum_{k=1}^{j-1} \frac{2(j-k)-1}{2 j-1} 2 \alpha_{q} U_{2 k-1}(1) l_{2(j-k)-1}(1) \\
& =-\frac{2 \alpha_{q}^{2 j-1}}{2 j-1}-2 \alpha_{q} U_{2 j-2}(1)+\frac{2 \alpha_{q}}{2 j-1} U_{2 j-2}(1) \\
& +\frac{2 \alpha_{q}}{2 j-1}\left(2(j-1) l_{2 j-2}+\sum_{k=1}^{2 j-3}(2 j-2-k) \alpha_{q} U_{k}(1) l_{2 j-2-k}(1)\right) \\
& =-\frac{2 \alpha_{q}^{2 j-1}}{2 j-1}-2 \alpha_{q} U_{2 j-2}(1)+\frac{2 \alpha_{q}}{2 j-1} U_{2 j-2}(1)+\frac{2 \alpha_{q}(2 j-2) U_{2 j-2}}{2 j-1}=-\frac{2 \alpha_{q}^{2 j-1}}{2 j-1} .
\end{aligned}
$$

Provaremos agora que $\phi_{q, 2 j-1}(1)=0$,

$$
\begin{aligned}
\phi_{q, 2 j-1}(1) & =-2 l_{2 j-1}(1)+l_{2 j-1}^{+}(1)+l_{2 j-1}^{-}(1) \\
& =\sum_{k=1}^{2 j-2} \frac{2 j-1-k}{2 j-1}\left(U_{k}(1)\left(2 l_{2 j-1-k}(1)-l_{2 j-1-k}^{+}(1)-l_{2 j-1-k}^{-}(1)\right)\right) \\
& -\sum_{k=1}^{2 j-2} \frac{2 j-1-k}{2 j-1} \alpha_{q} U_{k-1}(1)\left(l_{2 j-1-k}^{-}(1)-l_{2 j-1-k}^{+}(1)\right),
\end{aligned}
$$

usando a hipótese de indução e o que provamos anteriormente, temos

$$
\begin{aligned}
\phi_{q, 2 j-1}(1) & =\sum_{k=1}^{j} \frac{2 j-1-(2 k-1)}{2 j-1} U_{2 k-1}(1) \frac{\alpha(i)^{2(j-k)}}{j-k} \\
& -2 \sum_{k=1}^{j} \frac{2 j-1-2 k}{2 j-1} \alpha(i) U_{2 k-1}(1)\left(\frac{\alpha(i)^{2(j-k)-1}}{2(j-k)-1}\right)=0 .
\end{aligned}
$$

Para a diferença no caso par usaremos o caso ímpar e as hipóteses de indução, então

$$
\begin{aligned}
& l_{2 j}^{-}(1)-l_{2 j}^{+}(1)=U_{2 j}(1)-\alpha_{q} U_{2 j-1}(1)-U_{2 j}(1)-\alpha_{q} U_{2 j-1}(1) \\
& -\sum_{k=1}^{2 j-1} \frac{2 j-k}{2 j}\left(U_{k}(1)\left(l_{2 j-k}^{-}(1)-l_{2 j-k}^{+}(1)\right)\right) \\
& +\sum_{k=1}^{2 j-1} \frac{2 j-k}{2 j}\left(\alpha_{q} U_{k-1}(1)\left(l_{2 j-k}^{-}(1)+l_{2 j-k}^{+}(1)\right)\right) \\
& =-2 \alpha_{q} U_{2 j-1}(1)+\sum_{k=1}^{j-1} \frac{2 j-2 k}{2 j} \alpha_{q} U_{2 k-1}(1)\left(2 l_{2 j-2 k}(1)-\frac{\alpha_{q}^{2 j-2 k}}{j-k}\right) \\
& +\sum_{k=1}^{j} \frac{2(j-k)+1}{2 j}\left(U_{2 k-1}(1) \frac{2 \alpha_{q}^{2(j-k)+1}}{2(j-k)+1}+\alpha_{q} U_{2 k-2}(1) 2 l_{2(j-k)+1}(1)\right)
\end{aligned}
$$




$$
\begin{aligned}
& =-2 \alpha_{q} U_{2 j-1}(1)+\frac{2 \alpha_{q} U_{2 j-1}(1)}{2 j}+\frac{2 \alpha_{q}}{2 j}(2 j-1) l_{2 j-1}(1)+2 \alpha_{q} \sum_{k=2}^{2 j-1} \frac{2 j-k}{2 j} U_{k-1}(1) l_{2 j-k}(1) \\
& =-2 \alpha_{q} U_{2 j-1}(1)+\frac{\alpha_{q}}{j}\left(U_{2 j-1}(1)+(2 j-1) l_{2 j-1}(1)+\sum_{k=1}^{2 j-2}(2 j-1-k) U_{k}(1) l_{2 j-1-k}(1)\right) \\
& =-2 \alpha_{q} U_{2 j-1}(1)+\frac{\left(\alpha_{q}(2 j-1)+\alpha_{q}\right) U_{2 j-1}(1)}{j}=0 .
\end{aligned}
$$

Finalmente, para $\phi_{q, 2 j}(1)$, temos

$$
\begin{aligned}
& \phi_{q, 2 j}(1)=-2 l_{2 j}(1)+l_{2 j}^{+}(1)+l_{2 j}^{-}(1)+\frac{\alpha_{q}^{2 j}}{j} \\
& =\frac{\alpha_{q}^{2 j}}{j}+\sum_{k=1}^{2 j-1} \frac{2 j-k}{2 j}\left(U_{k}(1)\left(2 l_{2 j-k}(1)-l_{2 j-k}^{+}(1)-l_{2 j-k}^{-}(1)\right)\right) \\
& \left.-\sum_{k=1}^{2 j-1} \frac{2 j-k}{2 j} \alpha_{q} U_{k-1}(1)\left(l_{2 j-k}^{-}(1)-l_{2 j-k}^{+}(1)\right)\right) \\
& =\sum_{k=1}^{j-1} \frac{2 j-2 k}{2 j} U_{2 k}(1) \frac{\alpha_{q}^{2(j-k)}}{j-k}-2 \sum_{k=2}^{j} \frac{2 j-(2 k-1)}{2 j} \alpha_{q} U_{2 k-2}(1) \frac{\alpha_{q}^{2(j-k)+1}}{2(j-k)+1}=0,
\end{aligned}
$$

o que prova o lema.

Lema 22. Podemos representar as $\phi_{q, j}(w)$, para $0 \leq q \leq p-2$ e $j$ qualquer, da seguinte maneira,

$$
\begin{aligned}
\phi_{q, 2 j-1}(w) & =w^{2 j-2} \alpha_{q}^{2 j-2}\left(\phi_{q, 1}(w)\right)+\sum_{t=1}^{j-2} k_{2 j-1, t}(w) \alpha_{q}^{2 t}+2 \phi_{p-1,2 j-1}(w) \\
\phi_{q, 2 j}(w) & =-\frac{\left(w^{2 j}-1\right) \alpha_{q}^{2 j}}{j}+\sum_{t=1}^{j-1} k_{2 j, t}(w) \alpha_{q}^{2 t}+2 \phi_{p-1,2 j}(w),
\end{aligned}
$$

onde os $k_{j, t}(w)$ são polinômios em $w$.

Demonstração. A prova será feita por indução. As hipóteses consideradas para indução serão, para $1 \leq s \leq j-1$ :

$$
\begin{aligned}
\phi_{q, 2 s-1}(w) & =w^{2 s-2} \alpha_{q}^{2 s-2}\left(\phi_{q, 1}(w)\right)+\sum_{t=1}^{s-2} k_{2 s-1, t}(w) \alpha_{q}^{2 t}+2 \phi_{p-1,2 s-1}(w), \\
\phi_{q, 2 s}(w) & =-\frac{w^{2 s} \alpha_{q}^{2 s}}{s}+\sum_{t=1}^{s-1} k_{2 s, t}(w) \alpha_{q}^{2 t}+2 \phi_{p-1,2 s}(w), \\
l_{2 s-1}^{+}(w)-l_{2 s-1}^{-}(w) & =\frac{2}{2 s-1} \alpha_{q}^{2 s-1} w^{2 s-1}+\alpha_{q} \sum_{t=0}^{s-2} d_{2 s-1, t}(w) \alpha_{q}^{2 t}, \\
l_{2 s}^{+}(w)-l_{2 s}^{-}(w) & =-\alpha_{q}^{2 s-1} w^{2 s-1} \phi_{q, 1}(w)+\alpha_{q} \sum_{t=0}^{s-2} d_{2 s, t}(w) \alpha_{q}^{2 t},
\end{aligned}
$$


onde $d_{s, t}$ são polinômios em $w$ e usamos a definição de $l_{2 s-1}^{ \pm}(w)$ e $l_{2 s}^{ \pm}(w)$ para determinar as hipóteses (6.16) e (6.17).

Para $s=1$ temos

$$
\begin{aligned}
\phi_{q, 1}(w) & =2 \phi_{p-1,1}(w)=-w+w^{3} \\
\phi_{q, 2}(w) & =-\left(w^{2}-1\right) \alpha_{q}^{2}+2 \phi_{p-1,2}(w)=-\left(w^{2}-1\right) \alpha_{q}^{2}+\left(-\frac{w^{2}}{2}+2 w^{4}-\frac{3 w^{6}}{2}\right) \\
l_{1}^{+}(w)-l_{1}^{-}(w) & =2 \alpha_{q} w \\
l_{2}^{+}(w)-l_{2}^{-}(w) & =-\alpha_{q} w \phi_{q, 1}(w) .
\end{aligned}
$$

Suponhamos que as fórmulas sejam válidas para $1,2, \ldots, j-1$. Provaremos que as mesmas valem para $s=j$. Usaremos uma idéia similar a utilizada na prova do lema anterior. Primeiro provaremos as fórmulas com índices ímpares e depois provaremos para os índices pares. Usando as hipóteses de indução, temos

$$
\begin{aligned}
& l_{2 j-1}^{+}(w)-l_{2 j-1}^{-}(w)=2 \alpha_{q} U_{2 j-2}(w)-\sum_{k=1}^{2 j-2} \frac{2 j-1-k}{2 j-1} V_{k}(w)\left(l_{2 j-1-k}^{+}(w)-l_{2 j-1-k}^{-}(w)\right) \\
& +\sum_{k=1}^{2 j-2} \frac{2 j-1-k}{2 j-1} w \alpha_{q} U_{k-1}(w)\left(l_{2 j-1-k}^{+}(w)+l_{2 j-1-k}^{-}(w)\right) \\
& =2 \alpha_{q} U_{2 j-2}(w)-\sum_{k=1}^{j-1} \frac{2 j-1-2 k}{2 j-1} V_{2 k}(w)\left(l_{2 j-1-2 k}^{+}(w)-l_{2 j-1-2 k}^{-}(w)\right) \\
& -\sum_{k=1}^{j-1} \frac{2 j-1-2 k}{2 j-1} w \alpha_{q} U_{2 k-1}(w)\left(l_{2 j-1-2 k}^{+}(w)+l_{2 j-1-2 k}^{-}(w)\right) \\
& -\sum_{k=1}^{j-1} \frac{2 j-2 k}{2 j-1}\left(V_{2 k-1}(w)\left(l_{2 j-2 k}^{+}(w)-l_{2 j-2 k}^{-}(w)\right)+w \alpha_{q} U_{2 k-2}(w)\left(l_{2 j-2 k}^{+}(w)+l_{2 j-2 k}^{-}(w)\right)\right) \\
& =\frac{2}{2 j-1} \alpha_{q}^{2 j-1} w^{2 j-1}+\alpha_{q} \sum_{t=0}^{j-2} d_{2 j-1, t}(w) \alpha_{q}^{2 t} .
\end{aligned}
$$

Para $\phi_{q, 2 j-1}(w)$, usando as hipóteses de indução, temos

$$
\begin{aligned}
& \phi_{q, 2 j-1}(w)=-2 l_{2 j-1}(w)+l_{2 j-1}^{+}(w)+l_{2 j-1}^{-}(w) \\
& =-2 U_{2 j-1}(w)+2 V_{2 j-1}(w)+\sum_{k=1}^{2 j-2} \frac{2 j-1-k}{2 j-1}\left(2 U_{k} l_{2 j-1-k}(w)\right) \\
& -\sum_{k=1}^{2 j-2} \frac{2 j-1-k}{2 j-1}\left(V_{k}\left(l_{2 j-1-k}^{+}(w)+l_{2 j-1-k}^{-}(w)\right)+p \alpha(i) U_{k-1}\left(l_{2 j-1-k}^{+}-l_{2 j-1-k}^{-}\right)\right)
\end{aligned}
$$




$$
\begin{aligned}
& =-2 U_{2 j-1}(w)+2 V_{2 j-1}(w)+\sum_{k=1}^{2 j-2} \frac{2 j-1-k}{2 j-1}\left(2 U_{k}(w) l_{2 j-1-k}(w)\right) \\
& -\sum_{k=1}^{j-1} \frac{2 j-1-2 k}{2 j-1}\left(V_{2 k}(w)\left(l_{2 j-1-2 k}^{+}(w)+l_{2 j-1-2 k}^{-}(w)\right)\right) \\
& -\sum_{k=1}^{j-1} \frac{2 j-1-2 k}{2 j-1}\left(w \alpha_{q} U_{2 k-1}(w)\left(l_{2 j-1-2 k}^{+}(w)-l_{2 j-1-2 k}^{-}(w)\right)\right) \\
& -\sum_{k=1}^{j-1} \frac{2 j-2 k}{2 j-1}\left(V_{2 k-1}(w)\left(l_{2 j-2 k}^{+}(w)+l_{2 j-2 k}^{-}(w)\right)+w \alpha_{q} U_{2 k-2}(w)\left(l_{2 j-2 k}^{+}(w)-l_{2 j-2 k}^{-}(w)\right)\right) \\
& =w^{2 j-2} \alpha_{q}^{2 j-2}\left(\phi_{q, 1}(w)\right)+\sum_{t=1}^{j-2} k_{2 j-1, t}(w) \alpha_{q}^{2 t}+2 \phi_{p-1,2 j-1}(w) .
\end{aligned}
$$

No caso da diferença de índice par, usando o caso ímpar e as hipóteses de indução, temos

$$
\begin{aligned}
& l_{2 j}^{+}(w)-l_{2 j}^{-}(w)=2 \alpha_{q} U_{2 j-1}(w)-\sum_{k=1}^{j-1} \frac{2 j-2 k}{2 j} V_{2 k}(w)\left(l_{2 j-2 k}^{+}(w)-l_{2 j-2 k}^{-}(w)\right) \\
& -\sum_{k=1}^{j-1} \frac{2 j-2 k}{2 j} w \alpha_{q} U_{2 k-1}(w)\left(l_{2 j-2 k}^{+}(w)+l_{2 j-2 k}^{-}(w)\right) \\
& -\sum_{k=1}^{j-1} \frac{2 j-2 k+1}{2 j} V_{2 k-1}(w)\left(l_{2 j-2 k+1}^{+}(w)-l_{2 j-2 k+1}^{-}(w)\right) \\
& -\sum_{k=1}^{j-1} \frac{2 j-2 k+1}{2 j} w \alpha_{q} U_{2 k-2}(w)\left(l_{2 j-2 k+1}^{+}(w)+l_{2 j-2 k+1}^{-}(w)\right) \\
& =-\alpha_{q}^{2 j-1} w^{2 j-1} \phi_{q, 1}(w)+\alpha_{q} \sum_{t=0}^{j-2} d_{2 j, t}(w) \alpha_{q}^{2 t} .
\end{aligned}
$$

Seguindo a mesma idéia para $\phi_{q, 2 j}(w)$ temos

$$
\phi_{q, 2 j}(w)=-\frac{\left(w^{2 j}-1\right) \alpha_{q}^{2 j}}{j}+\sum_{t=1}^{j-1} k_{2 j, t}(w) \alpha_{q}^{2 t}+2 \phi_{p-1,2 j}(w) .
$$

E o resultado segue.

Corolário 5. Para todo $j \geq 1$, a Expansão de Laurent das funções $\Phi_{q, 2 j+1}(s)$ em $s=0$ possuem os coeficientes:

$$
\begin{aligned}
& \operatorname{Res}_{s=0} \Phi_{q, 2 j+1}(s)=\frac{2}{2 j+1} \alpha_{q}^{2 j}+\sum_{t=1}^{j-1} K_{2 j+1, t} \alpha_{q}^{2 t}+2 \operatorname{Res}_{z=0} \Phi_{2 j+1, p-1}(s), \\
& \underset{\operatorname{Res}_{1}}{s=0} \Phi_{q, 2 j+1}(s)=0,
\end{aligned}
$$

onde $K_{2 j-1, t}$ são números reais. Em particular, quando $j=0$, temos

$$
\operatorname{Res}_{s=0} \Phi_{q, 1}(s)=2 \operatorname{Res}_{s=0} \Phi_{p-1,1}(s)=2, \quad \operatorname{Res}_{s=0} \Phi_{q, 1}(s)=0 .
$$


Demonstração. Pelo lema 21 segue-se que,

$$
\phi_{q, 2 j+1}(\lambda)=\sum_{k=0}^{2 j+1} a_{q, 2 j+1, k} w^{2 k+2 j+1}
$$

onde $w=\frac{1}{\sqrt{1-\lambda}}$ e $\phi_{q, 2 j-1}(0)=0$. Então $\sum_{k=0}^{2 j-1} a_{q, 2 j-1, k}=0$. Agora usando a integral na equação (1.23), temos

$$
\Phi_{q, 2 j+1}(s)=\sum_{k=0}^{2 j+1} a_{q, 2 j+1, k} \frac{\Gamma\left(s+\frac{2(k+j)+1}{2}\right)}{s \Gamma\left(\frac{2(k+j)+1}{2}\right)} .
$$

Logo usando o resíduo que está na equação (‥6), temos que

$$
\operatorname{Res}_{s=0} \Phi_{q, 2 j-1}(s)=\sum_{k=0}^{2 j+1} a_{q, 2 j+1, k}=0 .
$$

Agora, usando o lema 22 e a integral na equação (1.23) obtemos, também,

$$
\Phi_{q, 2 j+1}(s)=\left(\frac{\Gamma\left(s+\frac{2 j+1}{2}\right)}{\Gamma\left(\frac{2 j+1}{2}\right) s}-\frac{\Gamma\left(s+\frac{2 j+3}{2}\right)}{\Gamma\left(\frac{2 j+3}{2}\right) s}\right) \alpha_{q}^{2 j}+\sum_{t=1}^{j-1} K_{2 j+1, t}(s) \alpha_{q}^{2 t}+2 \Phi_{p-1,2 j+1}(s),
$$

e assim

$$
\operatorname{Res}_{z=0} \Phi_{q, 2 j+1}(s)=\frac{2}{2 j+1} \alpha_{q}^{2 j}+\sum_{t=1}^{j-1} K_{2 j+1, t} \alpha_{q}^{2 t}+2 \operatorname{Res}_{z=0} \Phi_{p-1,2 j+1}(s) .
$$

Note que $K_{2 j+1, t}$ são números reais, como $k_{2 j+1, t}(w)$ são polinômios em $w$.

Vamos determinar agora os termos $A_{0,0}(0)$ e $A_{0,1}^{\prime}(0)$, definidos na equação (1.21) para as seqüências $S_{q}$ e $S_{q, \pm}$.

Lema 23. Para todo $0 \leq q \leq p-2$,

$$
\begin{aligned}
& \mathcal{A}_{q, 0,0}(s)=2 A_{q 0,0, q}(s)-A_{q, 0,0,+}(s)-A_{q, 0,0,-}(s)=-\sum_{n=1}^{\infty} \log \left(1-\frac{\alpha_{q}^{2}}{\mu_{q, n}^{2}}\right) \frac{m_{q, n}}{\mu_{q, n}^{2 s}} \\
& \mathcal{A}_{q, 0,1}(s)=2 A_{q 0,1, q}(s)-A_{q, 0,1,+}(s)-A_{q, 0,1,-}(s)=\zeta\left(2 s, U_{q}\right) .
\end{aligned}
$$

Demonstração. No caso de $S_{q}$ a equação (1.21) é da forma

$$
\begin{aligned}
& A_{q, 0,0}(s)=\sum_{n=1}^{\infty}\left(a_{q, 0,0, n}-\sum_{j=1}^{p} b_{q, 2 j-1,0,0} \mu_{q, n}^{-2 j+1}\right) \mu_{q, n}^{-2 s}, \\
& A_{q, 0,1}(s)=\sum_{n=1}^{\infty}\left(a_{q, 0,1, n}-\sum_{j=1}^{p} b_{q, 2 j-1,0,1} \mu_{q, n}^{-2 j+1}\right) \mu_{q, n}^{-2 s} .
\end{aligned}
$$


Para $S_{q, \pm}$ temos

$$
\begin{aligned}
& A_{q, 0,0, \pm}(s)=\sum_{n=1}^{\infty}\left(a_{p-1,0,0, n, \pm}-\sum_{j=1}^{p} b_{q, 2 j-1,0,0, \pm} \mu_{q, n}^{-2 j+1}\right) \mu_{q, n}^{-2 s}, \\
& A_{q, 0,1, \pm}(s)=\sum_{n=1}^{\infty}\left(a_{p-1,0,1, n, \pm}-\sum_{j=1}^{p} b_{q, 2 j-1,0,1, \pm} \mu_{q, n}^{-2 j+1}\right) \mu_{q, n}^{-2 s} .
\end{aligned}
$$

Precisamos da expansão para $\lambda$ suficientemente grande das funções $l_{2 j-1}(\lambda), l_{2 j-1}^{ \pm}(\lambda)$, para $j=1,2, \ldots, p, \log \Gamma\left(-\lambda, S_{q, n} / \mu_{q, n}^{2}\right)$ e $\log \Gamma\left(-\lambda, S_{q, \pm, n} / \mu_{q, n}^{2}\right)$. Para $S_{q}$, com as expansões que estão na equação (4.29), obtemos

$$
\begin{aligned}
\log \Gamma\left(-\lambda, S_{q, n} / \mu_{q, n}^{2}\right) & =\frac{1}{2} \log 2 \pi+\left(\mu_{q, n}+\frac{1}{2}\right) \log \mu_{q, n}-\mu_{q, n} \log 2 \\
& -\log \Gamma\left(\mu_{q, n}+1\right)+\frac{1}{2}\left(\mu_{q, n}+\frac{1}{2}\right) \log (-\lambda)+O\left(\mathrm{e}^{-\mu_{q, n} \sqrt{-\lambda}}\right) .
\end{aligned}
$$

No caso de $S_{q, \pm}$, usando as fórmulas da equação (4.29), temos que

$$
\hat{I}_{\nu, \pm \alpha_{q}}(z) \sim \frac{\sqrt{z} \mathrm{e}^{z}}{\sqrt{2 \pi}}\left(1+\sum_{k=1}^{\infty} b_{k} z^{-k}\right)+O\left(\mathrm{e}^{-z}\right)
$$

$\log 0$

$$
\begin{aligned}
\log \Gamma\left(-\lambda, S_{q, \pm, n} / \mu_{q, n}^{2}\right) & =\mu_{q, n} \sqrt{-\lambda}+\frac{1}{2} \log 2 \pi+\left(\mu_{q, n}-\frac{1}{2}\right) \log \mu_{q, n}-\mu_{q, n} \log 2 \\
- & \log \Gamma\left(\mu_{q, n}\right)+\frac{1}{2}\left(\mu_{q, n}-\frac{1}{2}\right) \log (-\lambda)+\log \left(1 \pm \frac{\alpha_{q}}{\mu_{q, n}}\right)+O\left(\mathrm{e}^{-\mu_{q, n} \sqrt{-\lambda}}\right) .
\end{aligned}
$$

Então temos os valores

$$
\begin{aligned}
& a_{q, 0,0, n}=\frac{1}{2} \log 2 \pi+\left(\mu_{q, n}+\frac{1}{2}\right) \log \mu_{q, n}-\mu_{q, n} \log 2-\log \Gamma\left(\mu_{q, n}+1\right), \\
& a_{q, 0,1, n}=\frac{1}{2}\left(\mu_{q, n}+\frac{1}{2}\right), \\
& a_{q, 0,0, n, \pm}=\frac{1}{2} \log 2 \pi+\left(\mu_{q, n}-\frac{1}{2}\right) \log \mu_{q, n}-\log 2^{\mu_{q, n}} \Gamma\left(\mu_{q, n}\right)+\log \left(1 \pm \frac{\alpha_{q}}{\mu_{q, n}}\right), \\
& a_{q, 0,1, n, \pm}=\frac{1}{2}\left(\mu_{q, n}-\frac{1}{2}\right),
\end{aligned}
$$

e ainda $b_{q, 2 j-1,0,0}, b_{q, 2 j-1,0,0, \pm}$ são todos nulos já que $l_{2 j-1}(\lambda)$ e $l_{2 j-1}^{ \pm}(\lambda)$ não possuem termos constantes. Assim,

$$
\begin{aligned}
& 2 a_{q, 0,0, n}-a_{q, 0,0, n,+}-a_{q, 0,0, n,-}=-\log \left(1-\frac{\alpha_{q}^{2}}{\mu_{q, n}^{2}}\right), \\
& 2 a_{q, 0,1, n}-a_{q, 0,1, n,+}-a_{q, 0,1, n,-}=1,
\end{aligned}
$$


e

$$
\begin{aligned}
& \mathcal{A}_{q, 0,0}(s)=2 A_{q, 0,0}(s)-A_{q, 0,0,+}(s)-A_{q, 0,0,-}(s)=-\sum_{n=1}^{\infty} \log \left(1-\frac{\alpha_{q}^{2}}{\mu_{q, n}^{2}}\right) \frac{m_{q, n}}{\mu_{q, n}^{2 s}}, \\
& \mathcal{A}_{q, 0,1}(s)=2 A_{q, 0,1}(s)-A_{q, 0,1,+}(s)-A_{q, 0,1,-}(s)=\zeta\left(2 s, U_{q}\right) .
\end{aligned}
$$

Proposição 15. As funçôes $t_{q}(s)$ e $t_{q}^{\prime}(s)$, para $0 \leq q \leq p-2$, em $s=0$ assumem os valores

$$
\begin{aligned}
& t_{q}(0)=-\zeta\left(0, U_{q}\right) \\
& t_{q}^{\prime}(0)=-\mathcal{A}_{q, 0,0}(0)-\mathcal{A}_{q, 0,1}^{\prime}(0)+\frac{1}{2} \sum_{j=0}^{p-1} \operatorname{Res}_{s=0} \Phi_{q, 2 j+1}(s) \operatorname{Res}_{s=2 j+1} \zeta\left(s, U_{q}\right) .
\end{aligned}
$$

Demonstração. Basta aplicar o teorema 5 e seu corolário junto com os lemas 18 e 23 e o corolário 5 .

Dividiremos $t_{q}(0)$ e $t_{q}^{\prime}(0)$ em duas partes como feito no estudo de $t_{p-1}(s)$, a saber,

$$
\begin{aligned}
& t_{q}(0)=t_{q, \mathrm{reg}}(0)+t_{q, \mathrm{sing}}(0) \\
& t_{q}^{\prime}(0)=t_{q, \mathrm{reg}}^{\prime}(0)+t_{q, \mathrm{sing}}^{\prime}(0),
\end{aligned}
$$

onde

$$
\begin{aligned}
t_{q, \mathrm{reg}}(0) & =-\zeta\left(0, U_{q}\right) \\
t_{q, \mathrm{sing}}(0) & =0 \\
t_{q, \mathrm{reg}}^{\prime}(0) & =-\mathcal{A}_{q, 0,0}(0)-\mathcal{A}_{q, 0,1}^{\prime}(0), \\
t_{q, \text { sing }}^{\prime}(0) & =\frac{1}{2} \sum_{j=0}^{p-1} \operatorname{Res}_{s=0} \Phi_{q, 2 j+1}(s) \operatorname{Res}_{s=2 j+1} \zeta\left(s, U_{q}\right) .
\end{aligned}
$$

\subsection{Torção Analítica de $C_{l} N$}

Lembramos a fórmula da Torção Analítica de $W=C_{l} N$ dada na seção 6.1

$$
\begin{aligned}
\log T(W)= & \frac{\log l^{2}}{2}\left(\sum_{q=0}^{p-1}(-1)^{q} t_{q}(0)+\sum_{q=0}^{p-1}(-1)^{q+1} \mathrm{rk}_{q}(N, \mathbb{R}) z_{q}(0)\right) \\
& +\frac{1}{2}\left(\sum_{q=0}^{p-1}(-1)^{q} t_{q}^{\prime}(0)+\sum_{q=0}^{p-1}(-1)^{q+1} \mathrm{rk}_{q}(N, \mathbb{R}) z_{q}^{\prime}(0)\right)
\end{aligned}
$$

Observamos que as funções $z_{q}(s)$ foram estudas em [41]. Podemos determinar facilmente $z_{q}(0)$ e $z_{q}^{\prime}(0)$ com as fórmulas na equação (1.22). Assim

$$
z_{q}(0)=-\frac{1}{2}, \quad z_{q}^{\prime}(0)=\log 2(p-q) .
$$


Seguindo a idéia para a parte regular e parte singular de $t_{q}(0)$ e $t_{q}^{\prime}(0)$, definimos

$$
\begin{array}{ll}
z_{q, \mathrm{reg}}(0)=z_{q}(0)=-\frac{1}{2}, & z_{q, \text { sing }}(0)=0, \\
z_{q, \mathrm{reg}}^{\prime}(0)=z_{q}^{\prime}(0)=\log 2(p-q), & z_{q, \text { sing }}^{\prime}(0)=0 .
\end{array}
$$

Com a divisão apresentada nas seções anteriores para $t_{q}(0)$ e $t_{q}^{\prime}(0)$, para $0 \leq q \leq p-1$, e agora para as $z_{q}(0)$ e $z_{q}^{\prime}(0)$, é conveniente separar o cálculo da Torção Analítica em parte regular $\left(\log T_{\text {reg }}(W)\right)$ e parte singular $\left(\log T_{\text {sing }}(W)\right)$ da seguinte forma

$$
\log T(W)=\log T_{\text {reg }}(W)+\log T_{\text {sing }}(W),
$$

com

$$
\begin{aligned}
\log T_{\mathrm{reg}}(W)= & \frac{\log l^{2}}{2}\left(\sum_{q=0}^{p-1}(-1)^{q+1} r_{q} z_{q}(0)+\sum_{q=0}^{p-1}(-1)^{q} t_{q, \mathrm{reg}}(0)\right) \\
& +\frac{1}{2}\left(\sum_{q=0}^{p-1}(-1)^{q+1} r_{q} z_{q}^{\prime}(0)+\sum_{q=0}^{p-1}(-1)^{q} t_{q, \mathrm{reg}}^{\prime}(0)\right), \\
\log T_{\text {sing }}(W)= & \frac{\log l^{2}}{2} \sum_{q=0}^{p-1}(-1)^{q} t_{q, \operatorname{sing}}(0)+\frac{1}{2} \sum_{q=0}^{p-1}(-1)^{q} t_{q, \text { sing }}^{\prime}(0),
\end{aligned}
$$

onde $r_{q}=\operatorname{rk} H_{q}(N, \mathbb{R})$.

Lema 24. A parte regular e a parte singular da Torção Analítica de $W$ possuem as formas a seguir:

$$
\begin{aligned}
& \log T_{\text {reg }}(W)=\frac{\log l^{2}}{2}\left(\sum_{q=0}^{p-1}(-1)^{q} \frac{r_{q}}{2}\right)+\frac{1}{2} \sum_{q=0}^{p-1}(-1)^{q+1} r_{q} \log 2(p-q)+\frac{1}{2} \log T\left(N, l^{2} \tilde{g}\right) \\
& \log T_{\text {sing }}(W)=\frac{1}{4} \sum_{q=0}^{p-1}(-1)^{q} \sum_{j=0}^{p-1} \operatorname{Res}_{s=0} \Phi_{q, 2 j+1}(s) \operatorname{Res}_{s=2 j+1} \zeta\left(s, U_{q}\right) .
\end{aligned}
$$

Demonstração. Usando o resultado para as funções $z_{q}(s)$, do início da seção e as proposições 13 e 15 temos

$$
\begin{array}{ll}
t_{q, \mathrm{reg}}(0)=-\zeta\left(0, U_{q}\right), & t_{q, \mathrm{reg}}^{\prime}(0)=-\mathcal{A}_{q, 0,0}(0)-\mathcal{A}_{q, 0,1}^{\prime}(0) \\
t_{p-1, \mathrm{reg}}(0)=-\frac{1}{2} \zeta\left(0, U_{p-1}\right), & t_{p-1, \mathrm{reg}}^{\prime}(0)=\zeta^{\prime}\left(0, U_{p}-1\right) \\
z_{k, \mathrm{reg}}(0)=-\frac{1}{2}, & z_{k, \mathrm{reg}}^{\prime}(0)=\log 2(p-k),
\end{array}
$$

onde $1 \leq q \leq p-2$ e $1 \leq k \leq p-1$.

Usando as representação de $\zeta\left(s, U_{q}\right)$, para $0 \leq q \leq p-2$, e de $\zeta\left(s, U_{p-1}\right)$, que estão nos lemas 18 e 13, respectivamente, obtemos

$$
t_{q, \mathrm{reg}}(0)=-\zeta\left(0, \tilde{U}_{q}\right), \quad t_{p-1, \mathrm{reg}}(0)=-\frac{1}{2} \zeta\left(0, \tilde{U}_{p-1}\right), \quad t_{p-1, \mathrm{reg}}^{\prime}(0)=\frac{1}{2} \zeta^{\prime}\left(0, \tilde{U}_{p}-1\right) .
$$


No caso de $t_{q, \text { reg }}^{\prime}(0)$, para $0 \leq q \leq p-2$ temos

$$
\begin{aligned}
-\mathcal{A}_{q, 0,0}(s)-\mathcal{A}_{q, 0,1}^{\prime}(s) & =\sum_{n=1}^{\infty} \frac{m_{q, n}}{\left(\mu_{q, n}\right)^{2 s}} \log \left(\mu_{q, n}\right)^{2}+\sum_{n=1}^{\infty} \log \left(1-\frac{\alpha_{q}^{2}}{\left(\mu_{q, n}\right)^{2}}\right) \frac{m_{q, n}}{\left(\mu_{q, n}\right)^{2 s}} \\
& =\sum_{n=1}^{\infty} \log \lambda_{q, n} \frac{m_{q, n}}{\left(\mu_{q, n}\right)^{2 s}} \\
& =\sum_{n=1}^{\infty} \log \lambda_{q, n} \sum_{j=0}^{\infty}\left(\begin{array}{c}
-s \\
j
\end{array}\right) \frac{m_{q, n}}{\left(\lambda_{q, n}\right)^{s+j}} \alpha_{q}^{2 j} \\
& =-\sum_{j=0}^{\infty}\left(\begin{array}{c}
-s \\
j
\end{array}\right) \zeta^{\prime}\left(s+j, \tilde{U}_{q}\right) \alpha_{q}^{2 j} .
\end{aligned}
$$

Portanto $t_{q, \text { reg }}^{\prime}(0)=-\zeta^{\prime}\left(0, \tilde{U}_{q}\right)$.

Agora,

$$
\begin{aligned}
& \log l^{2} \sum_{q=0}^{p-1}(-1)^{q} t_{q, \mathrm{reg}}(0)+\sum_{q=0}^{p-1}(-1)^{q} t_{q, \mathrm{reg}}^{\prime}(0)= \\
& =\log l^{2}\left(\sum_{q=0}^{p-2}(-1)^{q+1} \zeta\left(0, \tilde{U}_{q}\right)+\frac{(-1)^{p}}{2} \zeta\left(0, \tilde{U}_{p-1}\right)\right)+\sum_{q=0}^{p-2}(-1)^{q+1} \zeta^{\prime}\left(0, \tilde{U}_{q}\right)+\frac{(-1)^{p}}{2} \zeta^{\prime}\left(0, \tilde{U}_{p-1}\right) \\
& =\log T\left(N, l^{2} \tilde{g}\right) .
\end{aligned}
$$

Então

$$
\log T_{\mathrm{reg}}(W)=\frac{\log l^{2}}{2}\left(\sum_{q=0}^{p-1}(-1)^{q} \frac{r_{q}}{2}\right)+\frac{1}{2} \sum_{q=0}^{p-1}(-1)^{q+1} r_{q} \log 2(p-q)+\frac{1}{2} \log T\left(N, l^{2} \tilde{g}\right) .
$$

Para a parte singular basta substituir as fórmulas.

Como decorrência do lema temos

Teorema 19. A Torção Analítica do cone $C_{l} N$ de uma variedade fechada conexa $N$ de dimensão $2 p-1$ tem a forma

$$
\begin{aligned}
\log T\left(C_{l} N\right)=\frac{\log l^{2}}{2} & \left(\sum_{q=0}^{p-1}(-1)^{q} \frac{r_{q}}{2}\right)+\frac{1}{2} \log T\left(N, l^{2} \tilde{g}\right)+\frac{1}{2} \sum_{q=0}^{p-1}(-1)^{q+1} r_{q} \log 2(p-q) \\
& +\frac{1}{4} \sum_{q=0}^{p-1}(-1)^{q} \sum_{j=0}^{p-1} \operatorname{Res}_{s=0} \Phi_{q, 2 j+1}(s) \operatorname{Res}_{s=2 j+1} \zeta\left(s, U_{q}\right) .
\end{aligned}
$$

Nesse ponto um trabalho mais profundo é necessário. Afinal, precisaremos entender como estender a definição da Torção de Reidemeister para $C_{l} N$ para, então, poder escrever um teorema do tipo Cheeger Müller. Entender a construção da ferramenta de Brüning e Ma [4] é também uma peça chave para podermos determinar a contribuição do bordo na Torção 
Analítica por meio de ferramentas mais geométricas, o que seria muito mais interessante e, por fim, trabalhar o resíduo da função zeta de $U_{q}$ para comparar com a contribuição do bordo da fórmula de [4]. No caso da esfera de dimensão ímpar, todas estas determinações são possíveis, ou seja, podemos apresentar a contribuição do bordo em termos geométricos. Isto será feito na próxima seção.

\subsection{Prova da conjectura}

Consideraremos a partir de agora que $N=S_{\operatorname{sen} \alpha}^{2 p-1}$ e lembramos que $\nu=a^{-1}$, onde $a=\operatorname{sen} \alpha$. Os autovalores da esfera são apresentados nos artigos [23] e [50], lembramos aqui os autovalores das autoformas coexatas, com suas multiplicidades. Os autovalores das autoformas coexatas de $S_{\operatorname{sen} \alpha}^{2 p-1}$ são

$$
\left\{\begin{array}{l}
\lambda_{0, n}=\nu^{2} n(n+2 p-2), \\
\lambda_{q, n}=\nu^{2}(n+q)(n+2 p-q-2), \quad 1 \leq q<p-2, \\
\lambda_{p-2, n}=\nu^{2}\left((n-1+p)^{2}-1\right), \\
\lambda_{p-1, n}=\nu^{2}(n-1+p)^{2},
\end{array}\right.
$$

com as multiplicidades

$$
\begin{aligned}
& m_{0, n}=\frac{2}{(2 p-2) !} \prod_{j=2}^{p}(n-1+j)(2 p+n-1-j), \\
& m_{q, n}=\frac{2}{q !(2 p-q-2) !} \prod_{\substack{j=1, j \neq q+1}}^{p}(n-1+j)(2 p+n-1-j), 1 \leq q<p-2, \\
& m_{p-2, n}=\frac{2}{(p-2) ! p !} \prod_{\substack{j=1 \\
j \neq p-1}}^{p}(n-1+j)(2 p+n-1-j), \\
& m_{p-1, n}=\frac{2}{[(p-1) !]^{2}} \prod_{j=1}^{p-1}(n-1+j)(2 p+n-1-j),
\end{aligned}
$$

logo os índices $\mu_{q, n}$ tem a forma

$$
\left\{\begin{array}{l}
\mu_{0, n}=\sqrt{\nu^{2}(n(n+2 p-2))+(p-1)^{2}}, \\
\mu_{q, n}=\sqrt{\nu^{2}(n+q)(n+2 p-q-2)+\alpha(q)^{2}}, \quad 1 \leq q<p-2, \\
\mu_{p-2, n}=\sqrt{\nu^{2}\left((n-1+p)^{2}-1\right)+1}, \\
\mu_{p-1, n}=\nu(n-1+p) .
\end{array}\right.
$$

Usaremos ainda a notação $U_{q, S^{2 p-1}}=\left\{m_{q, n}: \lambda_{q, n, S^{2 p-1}}\right\}$ para representar os autovalores das autoformas coexatas de dimensão $q$ sobre a esfera de dimensão $2 p-1$ de raio 1 .

Faremos o estudo dos termos que faltam do teorema [19, ou seja, determinaremos os resíduos e os números que faltam para termos uma fórmula explícita para a Torção Analítica 
do cone sobre uma esfera de dimensão ímpar, de modo que, o resultado coincida com a conjectura 1 .

Antes de começarmos recordamos alguns fatos. Seja $a_{1}, \ldots, a_{m}$ uma seqüência de números reais, então podemos escrever o produto $\prod_{j=1}^{m}\left(x+a_{j}\right)$ da seguinte maneira

$$
\prod_{j=1}^{m}\left(x+a_{j}\right)=\sum_{j=0}^{m} e_{m-j}\left(a_{1}, \ldots, a_{m}\right) x^{j}
$$

onde $e_{1}, \ldots, e_{m}$ são polinômios elementares simétricos em $a_{1}, \ldots, a_{m}$. Definimos os números

$$
d_{j}^{q}:=(j-q-1)(2 p-q-j-1),
$$

para $q=0, \ldots, p-1, j \neq q+1$, e $d^{q}:=\left(d_{1}^{q}, d_{2}^{q}, \ldots, \hat{d}_{q+1}^{q}, \ldots, d_{n}^{q}\right)$, onde o. omissão do termo. Denotaremos por $\zeta_{R}(s)$ a função zeta de Riemann, ou seja,

$$
\zeta_{R}(s)=\sum_{n=1}^{\infty} \frac{1}{n^{s}}
$$

Lema 25. A seqüência $U_{p-1}$ é uma seqüência totalmente regular do tipo espectral com ordem infinita, $e\left(U_{p-1}\right)=g\left(U_{p-1}\right)=2 p-1 e$

$$
\zeta\left(s, U_{p-1}\right)=\frac{2 \nu^{-s}}{(p-1) !^{2}} \sum_{j=0}^{p-1} e_{p-1-j}\left(d^{p-1}\right) \zeta_{R}(s-2 j) .
$$

Demonstração. O expoente finito, o genus e o fato de $U_{p-1}$ ser uma seqüência totalmente regular do tipo espectral com ordem infinita seguem do lema 13 (a representação a seguir também nos garante o fato de ser uma seqüência espectral totalmente regular com ordem infinita). Para a representação de $\zeta\left(s, U_{p-1}\right)$, como acima, procedemos da seguinte maneira

$$
\zeta\left(s, U_{p-1}\right)=\nu^{-s} \zeta\left(\frac{s}{2}, U_{p-1, S^{2 p-1}}\right)
$$

onde

$$
\zeta\left(\frac{s}{2}, U_{p-1, S^{2 p-1}}\right)=\sum_{n=1}^{\infty} \frac{m_{p-1, n}}{\lambda_{p-1, n, S^{2 p-1}}^{\frac{s}{2}}}=\sum_{n=1}^{\infty} \frac{m_{p-1, n}}{(n+p-1)^{s}} .
$$

Transladando $n$ para $n-p+1$, na soma, e observando que $0,1, \ldots, p-1$ são raízes do polinômio $\sum_{j=0}^{p-1} e_{p-1-j}\left(d^{p-1}\right) n^{2 j}$, obtemos

$$
\begin{aligned}
\zeta\left(s, U_{p-1}\right) & =\nu^{-s} \sum_{n=p}^{\infty} \frac{m_{p-1, n-p+1}}{n^{s}} \\
& =\frac{2 \nu^{-s}}{(p-1) !^{2}} \sum_{n=p}^{\infty} \frac{\prod_{j=1}^{p-1} n^{2}-(p-j)^{2}}{n^{s}} \\
& =\frac{2 \nu^{-s}}{(p-1) !^{2}} \sum_{j=0}^{p-1} e_{p-1-j}\left(d^{p-1}\right) \zeta_{R}(s-2 j) .
\end{aligned}
$$


Observamos que, com a representação do lema anterior, $\zeta\left(s, U_{p-1}\right)$ possui uma expansão em série proximo a $s=2 k+1 \operatorname{com} k=0,1, \ldots, p-1$, da seguinte forma

$$
\zeta\left(s, U_{p-1}\right)=\frac{2}{\nu^{2 k+1}(p-1) !^{2}} e_{p-1-k}\left(d^{p-1}\right) \frac{1}{s-2 k-1}+L_{p-1,2 k+1}(s),
$$

onde $L_{p-1,2 k+1}(s)$ são funções regulares para $k=0,1, \ldots, p-1$.

Corolário 6. A função $\zeta\left(s, U_{p-1}\right)$ possui polos em $s=2 k+1$ para $k=0,1, \ldots, p-1 e$

$$
\operatorname{Res}_{s=2 k+1} \zeta\left(s, U_{p-1}\right)=\frac{2}{\nu^{2 k+1}(p-1) !^{2}} e_{p-1-k}\left(d^{p-1}\right) .
$$

Lema 26. A seqüência $U_{q}$ é uma seqüência totalmente regular do tipo espectral com ordem infinita, $\mathrm{e}\left(U_{q}\right)=\mathrm{g}\left(U_{q}\right)=2 p-1 e$

$$
\zeta\left(s, U_{q}\right)=\frac{2 \nu^{-s}}{q !(2 p-q-2) !} \sum_{t=0}^{\infty}\left(\begin{array}{c}
-\frac{s}{2} \\
t
\end{array}\right) \sum_{j=0}^{p-1} e_{p-1-j}\left(d^{q}\right) z\left(\frac{s+2 t-2 j}{2}, \alpha_{q} \mathrm{i}\right) \frac{\alpha_{q}^{2} t}{\nu^{2 t}},
$$

onde,

$$
H_{q, 0}=\left\{m_{q, n}: \sqrt{\lambda_{q, n, S^{2 p-1}}}\right\}_{n=1}^{\infty},
$$

e $\lambda_{q, n, S^{2 p-1}}$ é um autovalor de uma autoforma coexata de $S^{2 p-1}$ de dimensão $q$, e $q=$ $0,1, \ldots, p-2$. Além disso, $\zeta\left(s, U_{q}\right)$ possui polos simples em $s=2(p-k)-1$ para $k=0,1,2, \ldots$ Demonstração. O expoente finito, os polos e o fato de $U_{q}$ ser uma seqüência totalmente regular do tipo espectral com ordem infinita, seguem pelo mesmo argumento do lema13. Junto com a prova da representação de $\zeta\left(s, U_{q}\right)$, como no enunciado, mostraremos ainda a afirmação sobre os polos. Considere a seqüência

$$
H_{q, h}=\left\{m_{q, n}: \sqrt{\lambda_{q, n, S^{2 p-1}}+h}\right\}_{n=1}^{\infty},
$$

onde $\lambda_{q, n, S^{2 p-1}}$ é um autovalor de uma autoforma coexata de $S^{2 p-1}$ de dimensão $q$. Claramente,

$$
\zeta\left(s, U_{q}\right)=\nu^{-s} \zeta\left(s, H_{q, \frac{\alpha_{q}^{2}}{\nu^{2}}} .\right.
$$

Agora,

$\zeta\left(s, H_{q, h}\right)=\sum_{n=1}^{\infty} \frac{m_{q, n}}{\left(\lambda_{q, n, S^{2 p-1}}+h\right)^{\frac{s}{2}}}=\sum_{n=1}^{\infty} \sum_{t=0}^{\infty}\left(\begin{array}{c}-\frac{s}{2} \\ t\end{array}\right) \frac{m_{q, n}}{\lambda_{q, n, S^{2 p-1}}^{\frac{s}{2}+t}} h^{t}=\sum_{t=0}^{\infty}\left(\begin{array}{c}-\frac{s}{2} \\ t\end{array}\right) \zeta\left(s+2 t, H_{q, 0}\right) h^{t}$, e então $\zeta\left(s, U_{q}\right)$ tem a representação do enunciado.

A função zeta da seqüência $H_{q, 0}$ tem a forma

$$
\begin{aligned}
\zeta\left(2 s, H_{q, 0}\right) & =\zeta\left(s, U_{q, S^{2 p-1}}\right)=\sum_{n=1}^{\infty} \frac{m_{q, n}}{\lambda_{q, n, S^{2 p-1}}^{s}} \\
& =\sum_{n=p}^{\infty} \frac{m_{q, n-p+1}}{\lambda_{q, n-p+1, S^{2 p-1}}^{s}} \\
& =\frac{2}{q !(2 p-q-2) !} \sum_{n=p}^{\infty} \frac{\prod_{\substack{j=1, j \neq q+1}}^{p}\left(n^{2}-(p-j)^{2}\right)}{\left(n^{2}-\alpha_{q}^{2}\right)} .
\end{aligned}
$$


Lembramos que $\alpha_{q}^{2}=d_{p}^{q}$ e observamos que

$$
\begin{aligned}
\sum_{j=0}^{p-1} e_{p-j-1}\left(d^{q}\right)\left(n^{2}-\alpha_{q}^{2}\right)^{j} & =\sum_{j=0}^{p-1} e_{p-j-1}\left(d^{q}\right)\left(n^{2}-d_{p}^{q}\right)^{j}=\prod_{\substack{j=1, j \neq q+1}}^{p}\left(n^{2}-d_{p}^{q}+d_{j}^{q}\right) \\
& =\prod_{\substack{j=1, j \neq q+1}}^{p}\left(n^{2}-(p-j)^{2}\right),
\end{aligned}
$$

e ainda $n=1,2, \ldots,-\alpha_{q}$ são raízes do polinômio acima. Logo, podemos escrever $\zeta\left(2 s, H_{q, 0}\right)$ da seguinte forma,

$$
\begin{aligned}
\zeta\left(2 s, H_{q, 0}\right) & =\frac{2}{q !(2 p-q-2) !} \sum_{j=0}^{p-1} e_{p-1-j}\left(d^{q}\right)\left(z\left(s-j, \alpha_{q} \mathrm{i}\right)-\sum_{n=1}^{p-q-2}\left(n^{2}-\alpha_{q}^{2}\right)^{-s+j}\right) \\
& =\frac{2}{q !(2 p-q-2) !} \sum_{j=0}^{p-1} e_{p-1-j}\left(d^{q}\right) z\left(s-j, \alpha_{q} \mathrm{i}\right),
\end{aligned}
$$

onde $\mathrm{i}=\sqrt{-1}$ e a função $z\left(s-j, \alpha_{q} \mathrm{i}\right)$ é da forma

$$
z\left(s-j, \alpha_{q} \mathrm{i}\right)=\sum_{n=1}^{\infty} \frac{1}{\left(n^{2}-\alpha_{q}^{2}\right)^{s-j}} .
$$

Considere a função $z(s, a)=\sum_{n=1}^{\infty}\left(n^{2}+a^{2}\right)^{-s}$ e note que $z(s, 0)=\zeta_{R}(2 s)$. Portanto $z(s, 0)$ possui um único polo em $s=\frac{1}{2}$. Fazendo a expansão em $s$ da função $z(s, a)$ temos

$$
z(s, a)=\sum_{k=0}^{\infty}\left(\begin{array}{c}
-s \\
k
\end{array}\right) a^{2 k} \zeta_{R}(2 s+2 k) .
$$

Logo $z(s, a)$ possui polos simples quando $2 s+2 k=1$, ou seja, $s=\frac{1}{2}-k$, para $k=0,1,2, \ldots$. Como

$$
\zeta\left(2 s, H_{q, 0}\right)=\frac{2}{q !(2 p-q-2) !} \sum_{j=0}^{p-1} e_{p-1-j}\left(d^{q}\right) z\left(s-j, \alpha_{q} \mathrm{i}\right),
$$

$\zeta\left(2 s, H_{q, 0}\right)$ possui polos simples em $s=\frac{1}{2}+p-1-k$ para $k=0,1,2, \ldots$ Dessa maneira $\zeta\left(s, H_{q, 0}\right)$ possui polos simples em $s=2(p-k)-1$ para $k=0,1,2, \ldots$

Portanto $\zeta\left(s, U_{q}\right)$ tem a forma

$$
\zeta\left(s, U_{q}\right)=\frac{2 \nu^{-s}}{q !(2 p-q-2) !} \sum_{t=0}^{\infty}\left(\begin{array}{c}
-\frac{s}{2} \\
t
\end{array}\right) \sum_{j=0}^{p-1} e_{p-1-j}\left(d^{q}\right) z\left(\frac{s+2 t-2 j}{2}, \alpha_{q} \mathrm{i}\right) \frac{\alpha_{q}^{t}}{\nu^{2 t}},
$$

logo $\zeta\left(s, U_{q}\right)$ tem polos simples em $s=2(p-k)-1$, para $k=0,1,2, \ldots$

Corolário 7. A função $\zeta\left(s, U_{q}\right)$ possui polos simples em $s=2 k+1$, para $k=0,1, \ldots, p-1$, $e$

$$
\operatorname{Res}_{s=2 k+1} \zeta\left(s, U_{q}\right)=\frac{2 \nu^{-2 k-1}}{q !(2 p-q-2) !} \sum_{t=0}^{p-1-k} \frac{1}{\nu^{2 t}}\left(\begin{array}{c}
-\frac{2 k+1}{2} \\
t
\end{array}\right) \sum_{j=k+t}^{p-1} e_{p-1-j}\left(d^{q}\right)\left(\begin{array}{c}
-\frac{1}{2} \\
j-k-t
\end{array}\right) \alpha_{q}^{2(j-k)}
$$


Demonstração. Usaremos toda a notação do lema anterior. Precisamos apenas mostrar o valor do resíduo. Como o resíduo de grau 1 da função zeta de Riemann em $s=1$ é 1 temos

$$
\operatorname{Res}_{s=\frac{1}{2}-k} z(s, a)=\left(\begin{array}{c}
-\frac{1}{2}+k \\
k
\end{array}\right) a^{2 k} \operatorname{Res}_{s=\frac{1}{2}} \zeta_{R}(2 s)=\left(\begin{array}{c}
-\frac{1}{2}+k \\
k
\end{array}\right) \frac{a^{2 k}}{2},
$$

portanto,

$$
\operatorname{Res}_{s=\frac{1}{2}-k} z(s-j, a)=\operatorname{Res}_{s=\frac{1}{2}-j-k} z(s, a)=\left(\begin{array}{c}
-\frac{1}{2}+j+k \\
j+k
\end{array}\right) \frac{a^{2 j+2 k}}{2},
$$

para $k=0,1,2, \ldots$

Considerando $\zeta\left(2 s, H_{q, 0}\right)$ temos

$$
\begin{aligned}
\operatorname{Res}_{s=\frac{1}{2}+k} \zeta\left(2 s, H_{q, 0}\right) & =\frac{2}{q !(2 p-q-2) !} \sum_{j=0}^{p-1} e_{p-1-j}\left(d^{q}\right) \operatorname{Res}_{s=\frac{1}{2}+k} z\left(s-j, \alpha_{q} \mathrm{i}\right) \\
& =\frac{2}{q !(2 p-q-2) !} \sum_{j=k}^{p-1} e_{p-1-j}\left(d^{q}\right)(-1)^{j-k}\left(\begin{array}{c}
-\frac{1}{2}+j-k \\
j-k
\end{array}\right) \frac{\alpha_{q}^{2 j-2 k}}{2}
\end{aligned}
$$

para $k=0,1, \ldots, p-1 . \operatorname{Logo}$

$$
\operatorname{Res}_{s=2 k+1} \zeta\left(s, H_{q, 0}\right)=\frac{2}{q !(2 p-q-2) !} \sum_{j=k}^{p-1} e_{p-1-j}\left(d^{q}\right)(-1)^{j-k}\left(\begin{array}{c}
-\frac{1}{2}+j-k \\
j-k
\end{array}\right) \alpha_{q}^{2 j-2 k},
$$

para $k=0,1, \ldots, p-1$. Finalmente, para $\zeta\left(s, U_{q}\right)$ temos

$$
\operatorname{Res}_{s=2 k+1} \zeta\left(s, U_{q}\right)=\frac{2 \nu^{-2 k-1}}{q !(2 p-q-2) !} \sum_{t=0}^{p-1-k} \frac{1}{\nu^{2 t}}\left(\begin{array}{c}
-\frac{2 k+1}{2} \\
t
\end{array}\right) \sum_{j=k+t}^{p-1} e_{p-1-j}\left(d^{q}\right)\left(\begin{array}{c}
-\frac{1}{2} \\
j-k-t
\end{array}\right) \alpha_{q}^{2(j-k)} .
$$

Proposição 16. O logaritmo da Torção Analítica de $S_{\operatorname{sen} \alpha}^{2 p-1}$ com a métrica $l^{2} g_{S_{\operatorname{sen} \alpha}^{2 p-1}}$ é da forma

$$
\log T\left(S_{\operatorname{sen} \alpha}^{2 p-1}, l^{2} g_{S_{\operatorname{sen} \alpha}^{2 p-1}}\right)=\log \frac{2 l^{2 p-1} \pi^{p}}{\nu^{2 p-1}(p-1) !}
$$

Demonstração. Basta usar [15], $\log T\left(S_{\operatorname{sen} \alpha}^{2 p-1}, l^{2} g_{S_{\operatorname{sen} \alpha}^{2 p-1}}\right)=\log \tau\left(S_{l \operatorname{sen} \alpha}^{2 p-1}\right)=\operatorname{Vol}\left(S_{l \operatorname{sen} \alpha}^{2 p-1}\right), \log$ o

$$
\log T\left(S_{\operatorname{sen} \alpha}^{2 p-1}, l^{2} g_{S_{\operatorname{sen} \alpha}^{2 p-1}}\right)=\log \frac{2 l^{2 p-1} \pi^{p}}{\nu^{2 p-1}(p-1) !}
$$

Teorema 20. A parte regular da Torção Analítica do cone sobre uma esfera de dimensão impar é igual ao logaritmo da raiz quadrada do seu do volume,

$$
\log T_{\mathrm{reg}}(W)=\frac{1}{2} \log \operatorname{Vol} C_{\alpha} S_{l \operatorname{sen} \alpha}^{2 p-1} .
$$


Demonstração. Sabemos que

$$
\log T_{\mathrm{reg}}(W)=\frac{\log l^{2}}{2}\left(\frac{1}{2}\right)-\frac{1}{2} \log 2 p+\frac{1}{2} \log T\left(S_{\operatorname{sen} \alpha}^{2 p-1}, l^{2} g_{S_{\operatorname{sen} \alpha}^{2 p-1}}\right) .
$$

Usando a proposição 16 temos que,

$$
\log T_{\mathrm{reg}}(W)=\frac{1}{2} \log \frac{l^{2 p} \pi^{p}}{\nu^{2 p-1} p !}=\frac{1}{2} \log \operatorname{Vol}\left(C_{\alpha} S_{l \operatorname{sen} \alpha}^{2 p-1}\right)
$$

Faremos agora o estudo do termo singular da Torção Analítica do cone sobre as esferas de dimensão ímpar. Precisaremos introduzir uma notação nova.

Sejam

$$
\begin{aligned}
& D(q, k, t)=\frac{2}{q !(2 p-q-2) !}\left(\begin{array}{c}
-\frac{2 k+1}{2} \\
t
\end{array}\right) \sum_{l=k+t}^{p-1} e_{p-1-l}\left(d^{q}\right)(-1)^{l-k}\left(\begin{array}{c}
-\frac{1}{2}-k-t+l \\
l-k-t
\end{array}\right) \alpha_{q}^{2(l-k)}, \\
& F(q, k)=\operatorname{Res}_{s=0} \Phi_{q, 2 k+1}(s), \quad 1 \leq k \leq p-1,
\end{aligned}
$$

para $0 \leq q \leq p-1$ e, em particular, $F(q, 0)=2$ para $0 \leq q \leq p-2$ e $F(p-1,0)=1$. Então, usando o corolário 7 , os resíduos de $\zeta\left(s, U_{q}\right)$, para $0 \leq q \leq p-2$, tem a forma,

$$
\operatorname{Res}_{s=2 k+1} \zeta\left(s, U_{q}\right)=\frac{1}{\nu^{2 k+1}} \sum_{t=0}^{p-1-k} \frac{1}{\nu^{2 t}} D(q, k, t)
$$

para $k=0, \ldots, p-1$ e, quando $q=p-1$ usamos o corolário 6 e obtemos,

$$
\operatorname{Res}_{s=2 k+1} \zeta\left(s, U_{p-1}\right)=\frac{1}{\nu^{2 k+1}} D(p-1, k, 0)
$$

$\operatorname{com} k=0, \ldots, p-1$.

Agora, para $0 \leq q \leq p-1$, vemos que

$$
\operatorname{Res}_{s=0} \Phi_{q, 2 k+1}(s) \operatorname{Res}_{s=2 k+1} \zeta\left(s, U_{q}\right)=\frac{F(q, k)}{\nu^{2 k+1}} \sum_{t=0}^{p-1-k} \frac{1}{\nu^{2 t}} D(q, k, t)
$$

então

$$
t_{q, \text { sing }}^{\prime}(0)=\frac{1}{2} \sum_{k=0}^{p-1} \operatorname{Res}_{s=0} \Phi_{q, 2 k+1}(s) \operatorname{Res}_{s=2 k+1} \zeta\left(s, U_{q}\right)=\frac{1}{2} \sum_{k=0}^{p-1} \frac{F(q, k)}{\nu^{2 k+1}} \sum_{t=0}^{p-1-k} \frac{1}{\nu^{2 t}} D(q, k, t) .
$$

Chamaremos de termo singular da conjectura 1, e denotaremos por SingC, a parte que não envolve o volume do cone, ou seja,

$$
\operatorname{SingC}=\sum_{j=0}^{p-1} \frac{2^{p-j}}{j !(2(p-j)-1) ! !} \sum_{h=0}^{j}\left(\begin{array}{l}
j \\
h
\end{array}\right) \frac{(-1)^{h} \nu^{2(j-h)}}{(2(p-j+h)-1)} \frac{(2 p-1) !}{\nu^{2 p-1} 4^{p}(p-1) !},
$$


lembrando que $\nu=\frac{1}{\operatorname{sen} \alpha}$. Trabalharemos SingC no intuito de escrevê-lo como um polinômio em $\nu$, assim

$$
\begin{aligned}
\text { SingC } & =\sum_{j=0}^{p-1} \frac{2^{p-j}}{j !(2(p-j)-1) ! !} \sum_{h=0}^{j}\left(\begin{array}{l}
j \\
h
\end{array}\right) \frac{(-1)^{h} \nu^{-2(p-j+h)+1}}{(2(p-j+h)-1)} \frac{(2 p-1) !}{4^{p}(p-1) !} \\
& =\sum_{j=0}^{p-1} \frac{2^{j+1}}{(p-1-j) !(2 j+1) ! !} \sum_{h=0}^{p-1-j}\left(\begin{array}{c}
p-1-j \\
h
\end{array}\right) \frac{(-1)^{h} \nu^{-2(j+1+h)+1}}{2(j+1+h)-1} \frac{(2 p-1) !}{4^{p}(p-1) !} \\
& =\frac{(2 p-1) !}{4^{p}(p-1) !} \sum_{k=0}^{p-1} \frac{1}{(2 k+1) \nu^{2 k+1}} \sum_{j=0}^{k} \frac{(-1)^{k-j} 2^{j+1}}{(p-1-j) !(2 j+1) ! !}\left(\begin{array}{c}
p-1-j \\
k-j
\end{array}\right) \\
& =\frac{(2 p-1) !}{4^{p}(p-1) !} \sum_{k=0}^{p-1} \frac{1}{(p-1-k) !(2 k+1) \nu^{2 k+1}} \sum_{j=0}^{k} \frac{(-1)^{k-j} 2^{j+1}}{(k-j) !(2 j+1) ! !} .
\end{aligned}
$$

Agora escrevemos

$$
\operatorname{SingC}=\sum_{k=0}^{p-1} \frac{1}{\nu^{2 k+1}} \tilde{Q}_{p}(k), \quad \tilde{Q}_{p}(k)=\sum_{j=0}^{k} N_{j}(p, k),
$$

onde

$$
N_{j}(p, k)=\frac{(2 p-1) !}{4^{p}(p-1) !} \frac{1}{(p-1-k) !(2 k+1)} \frac{(-1)^{k-j} 2^{j+1}}{(k-j) !(2 j+1) ! !} .
$$

Dessa maneira, nosso objetivo é mostrar que SingC é igual a $\log T_{\operatorname{sing}}\left(C_{\alpha} S_{l \operatorname{sen} \alpha}^{2 p-1}\right)$, como no lema 24,

Lema 27. $\log T_{\operatorname{sing}}\left(C_{\alpha} S_{l \operatorname{sen} \alpha}^{2 p-1}\right)$ é um polinômio ímpar em $\nu^{-1}$.

Demonstração. A prova deste fato consiste apenas em escrever a fórmula da soma alternada e isolar $\nu$ como segue,

$$
\begin{aligned}
\frac{1}{2} \sum_{q=0}^{p-1}(-1)^{q} t_{q, \text { sing }}^{\prime}(0) & =\frac{1}{4} \sum_{q=0}^{p-1}(-1)^{q} \sum_{k=0}^{p-1} F(q, k) \sum_{t=0}^{p-1-k} \frac{1}{\nu^{2(t+k)+1}} D(q, k, t) \\
& =\frac{1}{4} \sum_{k=0}^{p-1} \frac{1}{\nu^{2 k+1}} \sum_{q=0}^{p-1}(-1)^{q} \sum_{j=0}^{k} F(q, j) D(q, j, k-j) \\
& =\frac{1}{4} \sum_{k=0}^{p-1} \frac{1}{\nu^{2 k+1}} \sum_{j=0}^{k} \sum_{q=0}^{p-1}(-1)^{q} F(q, j) D(q, j, k-j) .
\end{aligned}
$$

Com isto, escrevemos

$$
\frac{1}{2} \sum_{q=0}^{p-1}(-1)^{q} t_{q, \text { sing }}^{\prime}(0)=\sum_{k=0}^{p-1} \frac{1}{\nu^{2 k+1}} Q_{p}(k), \quad Q_{p}(k)=\sum_{j=0}^{k} M_{j}(p, k)
$$


onde

$$
\begin{aligned}
M_{j}(p, k) & =\sum_{q=0}^{p-1}(-1)^{q} F(q, j) D(q, j, k-j) \\
& =\sum_{q=0}^{p-1}(-1)^{q} \frac{2 F(q, j)}{4(2 p-2) !}\left(\begin{array}{c}
2 p-2 \\
q
\end{array}\right)\left(\begin{array}{c}
-\frac{1}{2}-j \\
k-j
\end{array}\right) \alpha_{q}^{-2 j} \sum_{l=k}^{p-1} e_{p-1-l}\left(d^{q}\right) \alpha_{q}^{2 l}\left(\begin{array}{c}
-\frac{1}{2} \\
l-k
\end{array}\right),
\end{aligned}
$$

e então basta provar que $M_{j}(p, k)=N_{j}(p, k)$.

Antes, precisaremos de algumas equações. Primeiro, lembremos que, se

$$
f_{h}(x)=e_{h}\left(x^{2}-(p-1)^{2}, x^{2}-(p-2)^{2}, \ldots, x^{2}-1^{2}, x^{2}\right)
$$

então $f_{h}\left(\alpha_{q}\right)=e_{h}\left(d^{q}\right)$ e $f_{h}(x)$, para $h \geq 1$, é um polinômio da forma

$$
f_{h}(x)=\sum_{0 \leq j_{1} \leq j_{2} \leq \ldots \leq j_{h} \leq p-1}\left(x^{2}-j_{1}^{2}\right)\left(x^{2}-j_{2}^{2}\right) \ldots\left(x^{2}-j_{h}^{2}\right)=\left(\begin{array}{l}
p \\
h
\end{array}\right) x^{2 h}+\sum_{s=0}^{h-1} c_{s}^{h} x^{2 s} .
$$

Segundo, precisaremos de quatro igualdades importantes. As três primeiras encontram-se no livro [21], são as equações $0.151-4,0.154-5$ e $0.154-6$ e as provas podem ser encontradas em [25]. Reescreveremos elas aqui, respectivamente,

$$
\begin{aligned}
& \sum_{k=0}^{n} \frac{(-1)^{k}}{(2 n) !}\left(\begin{array}{c}
2 n \\
k
\end{array}\right)=\frac{(-1)^{n}}{(2 n) !}\left(\begin{array}{c}
2 n-1 \\
n
\end{array}\right)=\frac{(-1)^{n}}{2(2 n) !}\left(\begin{array}{c}
2 n \\
n
\end{array}\right) \\
& \sum_{k=0}^{n}(-1)^{n}\left(\begin{array}{l}
n \\
k
\end{array}\right)(\alpha+k)^{n}=(-1)^{n} n ! \\
& \sum_{k=0}^{N}(-1)^{n}\left(\begin{array}{c}
N \\
k
\end{array}\right)(\alpha+k)^{n-1}=0,
\end{aligned}
$$

onde $1 \leq n \leq N$ e $\alpha \in \mathbb{R}$. E a última equação necessária encontra-se no livro [22] equação (5.23),

$$
\sum_{l=0}^{n}\left(\begin{array}{c}
n+1 \\
l+1
\end{array}\right)\left(\begin{array}{c}
-\frac{1}{2} \\
l-k
\end{array}\right)=\left(\begin{array}{c}
n+\frac{1}{2} \\
n-k
\end{array}\right)=\frac{(2 n+1) ! !}{2^{n-k}(n-k) !(2 k+1) ! !} .
$$

Provaremos primeiro que $M_{0}(p, k)=N_{0}(p, k)$ e depois que $M_{j}(p, k)=N_{j}(p, k)$, para $j \geq 1$. Faremos isso em dois lemas.

Lema 28. Para $0 \leq k \leq p-1$, temos $M_{0}(p, k)=N_{0}(p, k)$.

Demonstração. Com $j=0$ temos

$$
\begin{aligned}
& M_{0}(p, k)=\sum_{q=0}^{p-1}(-1)^{q} \frac{2 F(q, 0)}{4(2 p-2) !}\left(\begin{array}{c}
2 p-2 \\
q
\end{array}\right)\left(\begin{array}{c}
-\frac{1}{2} \\
k
\end{array}\right) \sum_{l=k}^{p-1} e_{p-1-l}\left(d^{q}\right) \alpha_{q}^{2 l}\left(\begin{array}{c}
-\frac{1}{2} \\
l-k
\end{array}\right), \\
& N_{0}(p, k)=\frac{(2 p-1) !}{2^{2 p-1}(p-1) !} \frac{1}{(p-1-k) !(2 k+1)} \frac{(-1)^{k}}{k !}
\end{aligned}
$$


Primeiro, consideramos $k \neq 0$, temos:

$$
\begin{aligned}
M_{0}(p, k)= & \left(\begin{array}{c}
-\frac{1}{2} \\
k
\end{array}\right) \sum_{q=0}^{p-1}(-1)^{q} \frac{1}{(2 p-2) !}\left(\begin{array}{c}
2 p-2 \\
q
\end{array}\right) \sum_{l=k}^{p-1} f_{p-1-l}\left(\alpha_{q}\right) \alpha_{q}^{2 l}\left(\begin{array}{c}
-\frac{1}{2} \\
l-k
\end{array}\right) \\
= & \left(\begin{array}{c}
-\frac{1}{2} \\
k
\end{array}\right) \sum_{q=0}^{p-1}(-1)^{q} \frac{1}{(2 p-2) !}\left(\begin{array}{c}
2 p-2 \\
q
\end{array}\right) \sum_{l=k}^{p-1}\left(\begin{array}{c}
p \\
p-1-l
\end{array}\right) \alpha_{q}^{2 p-2-2 l} \alpha_{q}^{2 l}\left(\begin{array}{c}
-\frac{1}{2} \\
l-k
\end{array}\right) \\
+ & \left(\begin{array}{c}
-\frac{1}{2} \\
k
\end{array}\right) \sum_{q=0}^{p-1}(-1)^{q} \frac{1}{(2 p-2) !}\left(\begin{array}{c}
2 p-2 \\
q
\end{array}\right) \sum_{l=k}^{p-2} \sum_{s=0}^{p-2-l} c_{s}^{p-1-l} \alpha_{q}^{2 s} \alpha_{q}^{2 l}\left(\begin{array}{c}
-\frac{1}{2} \\
l-k
\end{array}\right) \\
= & \left(\begin{array}{c}
-\frac{1}{2} \\
k
\end{array}\right) \sum_{q=0}^{p-1}(-1)^{q} \frac{1}{(2 p-2) !}\left(\begin{array}{c}
2 p-2 \\
q
\end{array}\right) \alpha_{q}^{2 p-2} \sum_{l=k}^{p-1}\left(\begin{array}{c}
p \\
p-1-l
\end{array}\right)\left(\begin{array}{c}
-\frac{1}{2} \\
l-k
\end{array}\right) \\
+ & \sum_{l=k}^{p-2} \sum_{s=0}^{p-2-l} c_{s}^{p-1-l}\left(\begin{array}{c}
-\frac{1}{2} \\
k
\end{array}\right)\left(\begin{array}{c}
-\frac{1}{2} \\
l-k
\end{array}\right) \sum_{q=0}^{p-1}(-1)^{q} \frac{1}{(2 p-2) !}\left(\begin{array}{c}
2 p-2 \\
q
\end{array}\right) \alpha_{q}^{2 s+2 l} .
\end{aligned}
$$

Usando a terceira equação de (6.24) vemos que a segunda parte da soma é nula já que $2 s+2 l<$ $2 p-2$. Então

$$
\begin{aligned}
M_{0}(p, k) & =\left(\begin{array}{c}
-\frac{1}{2} \\
k
\end{array}\right) \sum_{q=0}^{p-1}(-1)^{q} \frac{1}{(2 p-2) !}\left(\begin{array}{c}
2 p-2 \\
q
\end{array}\right) \alpha_{q}^{2 p-2} \sum_{l=k}^{p-1}\left(\begin{array}{c}
p \\
p-1-l
\end{array}\right)\left(\begin{array}{c}
-\frac{1}{2} \\
l-k
\end{array}\right) \\
& =\frac{1}{2}\left(\begin{array}{c}
-\frac{1}{2} \\
k
\end{array}\right) \sum_{l=k}^{p-1}\left(\begin{array}{c}
p \\
p-1-l
\end{array}\right)\left(\begin{array}{c}
-\frac{1}{2} \\
l-k
\end{array}\right) \\
& =\frac{1}{2}\left(\begin{array}{c}
-\frac{1}{2} \\
k
\end{array}\right)\left(\begin{array}{c}
p \\
k+1
\end{array}\right) \frac{(k+1) !}{p !} \frac{(2 p-1) ! !}{(2 k+1) ! !} \frac{2^{k+1}}{2^{p}} \\
& =\frac{(-1)^{k}}{k !} \frac{1}{(p-k-1) !} \frac{(2 p-1) !}{(2 k+1)} \frac{1}{2^{2 p-1}(p-1) !}=N_{0}(p, k) .
\end{aligned}
$$

Agora, considerando $k=0$, temos

$$
\begin{aligned}
M_{0}(p, 0) & =\sum_{q=0}^{p-2}(-1)^{q} \frac{1}{(2 p-2) !}\left(\begin{array}{c}
2 p-2 \\
q
\end{array}\right) \sum_{l=0}^{p-1} f_{p-1-l}\left(\alpha_{q}\right) \alpha_{q}^{2 l}\left(\begin{array}{c}
-\frac{1}{2} \\
l
\end{array}\right)+\frac{1}{2} \\
& =\sum_{q=0}^{p-1}(-1)^{q} \frac{1}{(2 p-2) !}\left(\begin{array}{c}
2 p-2 \\
q
\end{array}\right) \sum_{l=0}^{p-1} f_{p-1-l}\left(\alpha_{q}\right) \alpha_{q}^{2 l}\left(\begin{array}{c}
-\frac{1}{2} \\
l
\end{array}\right)-1+\frac{1}{2} \\
& =\sum_{q=0}^{p-1}(-1)^{q} \frac{1}{(2 p-2) !}\left(\begin{array}{c}
2 p-2 \\
q
\end{array}\right) \sum_{l=0}^{p-1}\left(\begin{array}{c}
p \\
p-1-l
\end{array}\right) \alpha_{q}^{2 p-2}\left(\begin{array}{c}
-\frac{1}{2} \\
l
\end{array}\right) \\
& +\sum_{q=0}^{p-1}(-1)^{q} \frac{1}{(2 p-2) !}\left(\begin{array}{c}
2 p-2 \\
q
\end{array}\right) c_{0}^{p-1}-\frac{1}{2}
\end{aligned}
$$




$$
\begin{aligned}
= & \sum_{q=0}^{p-1}(-1)^{q} \frac{1}{(2 p-2) !}\left(\begin{array}{c}
2 p-2 \\
q
\end{array}\right) \sum_{l=0}^{p-1}\left(\begin{array}{c}
p \\
p-1-l
\end{array}\right) \alpha_{q}^{2 p-2}\left(\begin{array}{c}
-\frac{1}{2} \\
l
\end{array}\right) \\
+ & \frac{(-1)^{p-1}}{2(2 p-2) !}\left(\begin{array}{c}
2 p-2 \\
p-1
\end{array}\right)(-1)^{p-1}(p-1) !(p-1) !-\frac{1}{2} \\
= & \sum_{q=0}^{p-1}(-1)^{q} \frac{1}{(2 p-2) !}\left(\begin{array}{c}
2 p-2 \\
q
\end{array}\right) \sum_{l=0}^{p-1}\left(\begin{array}{c}
p \\
p-1-l
\end{array}\right) \alpha_{q}^{2 p-2}\left(\begin{array}{c}
-\frac{1}{2} \\
l
\end{array}\right)+\frac{1}{2}-\frac{1}{2} \\
= & \sum_{q=0}^{p-1}(-1)^{q} \frac{1}{(2 p-2) !}\left(\begin{array}{c}
2 p-2 \\
q
\end{array}\right) \alpha_{q}^{2 p-2} \sum_{l=0}^{p-1}\left(\begin{array}{c}
p \\
p-1-l
\end{array}\right)\left(\begin{array}{c}
-\frac{1}{2} \\
l
\end{array}\right) \\
= & \frac{1}{2} \sum_{l=0}^{p-1}\left(\begin{array}{c}
p \\
p-1-l
\end{array}\right)\left(\begin{array}{c}
-\frac{1}{2} \\
l
\end{array}\right)=\frac{1}{2^{p}} \frac{(2 p-1) ! !}{(p-1) !}=\frac{2 p-1}{2^{2 p-1}}\left(\begin{array}{c}
2 p-2 \\
p-1
\end{array}\right)=N_{0}(p, 0)
\end{aligned}
$$

Lema 29. Para $1 \leq j \leq p-1$, temos que $M_{j}(p, k)=N_{j}(p, k)$.

Demonstração. Note que $j \leq k$ e assim $1 \leq j \leq k \leq p-1$.

Lembramos que

$$
F(q, j)=\frac{2}{2 j+1} \alpha_{q}^{2 j}+\sum_{t=1}^{j-1} K_{2 j+1, t} \alpha_{q}^{2 t}+2 \underset{z=0}{\operatorname{Res}_{0}} \Phi_{p-1,2 j+1}(s),
$$

denote $K_{2 j+1,0}=2 \operatorname{Res}_{0 z=0} \Phi_{p-1,2 j+1}(s)$. Dividiremos em três casos. Primeiro, para $j=k<$ $p-1$, temos

$$
\begin{aligned}
M_{j}(p, j) & =\sum_{q=0}^{p-1}(-1)^{q} \frac{F(q, j)}{2(2 p-2) !}\left(\begin{array}{c}
2 p-2 \\
q
\end{array}\right) \sum_{l=j}^{p-1} e_{p-1-l}\left(d^{q}\right) \alpha_{q}^{2 l-2 j}\left(\begin{array}{c}
-\frac{1}{2} \\
l-j
\end{array}\right) \\
& =\sum_{q=0}^{p-1}(-1)^{q} \frac{\alpha_{q}^{2 j}}{(2 j+1)(2 p-2) !}\left(\begin{array}{c}
2 p-2 \\
q
\end{array}\right) \sum_{l=j}^{p-1} f_{p-1-l}\left(\alpha_{q}\right) \alpha_{q}^{2 l-2 j}\left(\begin{array}{c}
-\frac{1}{2} \\
l-j
\end{array}\right) \\
& +\sum_{t=0}^{j-1} K_{t, j} \sum_{q=0}^{p-1}(-1)^{q} \frac{\alpha_{q}^{2 t}}{(2 p-2) !}\left(\begin{array}{c}
2 p-2 \\
q
\end{array}\right) \sum_{l=j}^{p-1} f_{p-1-l}\left(\alpha_{q}\right) \alpha_{q}^{2 l-2 j}\left(\begin{array}{c}
-\frac{1}{2} \\
l-j
\end{array}\right)
\end{aligned}
$$

Usando a fórmula da equação (6.23) temos

$$
\begin{aligned}
M_{j}(p, j) & =\sum_{q=0}^{p-1}(-1)^{q} \frac{1}{(2 j+1)(2 p-2) !}\left(\begin{array}{c}
2 p-2 \\
q
\end{array}\right) \sum_{l=j}^{p-1}\left(\begin{array}{c}
p \\
p-1-l
\end{array}\right) \alpha_{q}^{2 p-2-2 l} \alpha_{q}^{2 l}\left(\begin{array}{c}
-\frac{1}{2} \\
l-j
\end{array}\right) \\
& +\sum_{q=0}^{p-1}(-1)^{q} \frac{1}{(2 j+1)(2 p-2) !}\left(\begin{array}{c}
2 p-2 \\
q
\end{array}\right) \sum_{l=j}^{p-2} \sum_{s=0}^{p-2-l} c_{s} \alpha_{q}^{2 s+2 l}\left(\begin{array}{c}
-\frac{1}{2} \\
l-j
\end{array}\right)
\end{aligned}
$$




$$
\begin{aligned}
& +\sum_{t=0}^{j-1} K_{2 j+1, t} \sum_{q=0}^{p-1}(-1)^{q} \frac{1}{(2 p-2) !}\left(\begin{array}{c}
2 p-2 \\
q
\end{array}\right) \sum_{l=j}^{p-1}\left(\begin{array}{c}
p \\
p-1-l
\end{array}\right) \alpha_{q}^{2 p-2+2 t-2 j}\left(\begin{array}{c}
-\frac{1}{2} \\
l-j
\end{array}\right) \\
& +\sum_{t=0}^{j-1} K_{2 j+1, t} \sum_{q=0}^{p-1}(-1)^{q} \frac{1}{(2 p-2) !}\left(\begin{array}{c}
2 p-2 \\
q
\end{array}\right) \sum_{l=j}^{p-2} \sum_{s=0}^{p-2-l} c_{s} \alpha_{q}^{2 s+2 l+2 t-2 j}\left(\begin{array}{c}
-\frac{1}{2} \\
l-j
\end{array}\right) \\
& =\frac{1}{(2 j+1)} \sum_{q=0}^{p-1}(-1)^{q} \frac{1}{(2 p-2) !}\left(\begin{array}{c}
2 p-2 \\
q
\end{array}\right) \alpha_{q}^{2 p-2} \sum_{l=j}^{p-1}\left(\begin{array}{c}
p \\
p-1-l
\end{array}\right)\left(\begin{array}{c}
-\frac{1}{2} \\
l-j
\end{array}\right) \\
& =\frac{1}{2(2 j+1)} \sum_{l=j}^{p-1}\left(\begin{array}{c}
p \\
p-1-l
\end{array}\right)\left(\begin{array}{c}
-\frac{1}{2} \\
l-j
\end{array}\right)=\frac{1}{2(2 j+1)} \frac{1}{(p-1-j) !} \frac{(2 p-1) ! !}{(2 j+1) ! !} \frac{2^{j+1}}{2^{p}} \\
& =\frac{1}{(2 j+1)(p-1-j) !} \frac{(2 p-1) !}{(2 j+1) ! !} \frac{2^{j}}{2^{2 p-1}} \frac{1}{(p-1) !}=N_{j}(p, j) .
\end{aligned}
$$

Observamos os três últimos termos na primeira igualdade são nulos, já que $s+l<p-1$ e $t-j \leq-1$.

O segundo caso é $j=k=p-1$. Temos

$$
\begin{aligned}
M_{p-1}(p, p-1) & =\sum_{q=0}^{p-1}(-1)^{q} \frac{F(q, p-1)}{2(2 p-2) !}\left(\begin{array}{c}
2 p-2 \\
q
\end{array}\right) \\
& =\sum_{q=0}^{p-1}(-1)^{q} \frac{\alpha_{q}^{2 p-2}}{(2 p-1)(2 p-2) !}\left(\begin{array}{c}
2 p-2 \\
q
\end{array}\right) \\
& +\sum_{t=0}^{p-2} K_{2 j+1, t} \sum_{q=0}^{p-2}(-1)^{q} \frac{\alpha_{q}^{2 t}}{2(2 p-2) !}\left(\begin{array}{c}
2 p-2 \\
q
\end{array}\right) \\
& +\sum_{t=0}^{p-2} \frac{K_{2 j+1, t}}{2}(-1)^{p-1} \frac{\alpha_{p-1}^{2 t}}{2(2 p-2) !}\left(\begin{array}{c}
2 p-2 \\
p-1
\end{array}\right) \\
& =\frac{1}{2(2 p-1)}+K_{2 j+1,0} \sum_{q=0}^{p-1)^{q}} \frac{1}{2(2 p-2) !}\left(\begin{array}{c}
2 p-2 \\
q
\end{array}\right) \\
& -K_{2 j+1,0}(-1)^{p-1} \frac{1}{2(2 p-2) !}\left(\begin{array}{c}
2 p-2 \\
p-1
\end{array}\right)+\frac{K_{2 j+1,0}}{2}(-1)^{p-1} \frac{1}{2(2 p-2) !}\left(\begin{array}{c}
2 p-2 \\
p-1
\end{array}\right) \\
& =\frac{1}{2(2 p-1)}+\frac{K_{2 j+1,0}}{2} \frac{(-1)^{p-1}}{(p-1) !(p-1) !}-\frac{K_{2 j+1,0}}{2} \frac{(-1)^{p-1}}{(p-1) !(p-1) !} \\
& =\frac{1}{2(2 p-1)}=N_{p-1}(p, p-1) .
\end{aligned}
$$

Finalmente, para $1 \leq j<k$, temos

$$
M_{j}(p, k)=\sum_{i=0}^{p-1}(-1)^{q} \frac{2 F(q, j)}{4(2 p-2) !}\left(\begin{array}{c}
2 p-2 \\
q
\end{array}\right)\left(\begin{array}{c}
-\frac{1}{2}-j \\
k-j
\end{array}\right) \alpha_{q}^{-2 j} \sum_{l=k}^{p-1} e_{p-1-l}\left(d^{q}\right) \alpha_{q}^{2 l}\left(\begin{array}{c}
-\frac{1}{2} \\
l-k
\end{array}\right)
$$




$$
\begin{aligned}
& =\left(\begin{array}{c}
-\frac{1}{2}-j \\
k-j
\end{array}\right) \sum_{q=0}^{p-1}(-1)^{q} \frac{1}{(2 j+1)(2 p-2) !}\left(\begin{array}{c}
2 p-2 \\
q
\end{array}\right) \sum_{l=k}^{p-1}\left(\begin{array}{c}
p \\
p-1-l
\end{array}\right) \alpha_{q}^{2 p-2-2 l} \alpha_{q}^{2 l}\left(\begin{array}{c}
-\frac{1}{2} \\
l-k
\end{array}\right) \\
& +\left(\begin{array}{c}
-\frac{1}{2}-j \\
k-j
\end{array}\right) \sum_{q=0}^{p-1}(-1)^{q} \frac{1}{(2 j+1)(2 p-2) !}\left(\begin{array}{c}
2 p-2 \\
q
\end{array}\right) \sum_{l=k}^{p-2} \sum_{s=0}^{p-2-l} c_{s} \alpha_{q}^{2 s+2 l}\left(\begin{array}{c}
-\frac{1}{2} \\
l-k
\end{array}\right) \\
& +\left(\begin{array}{c}
-\frac{1}{2}-j \\
k-j
\end{array}\right) \sum_{t=0}^{j-1} K_{2 j+1, t} \sum_{q=0}^{p-1}(-1)^{q} \frac{1}{(2 p-2) !}\left(\begin{array}{c}
2 p-2 \\
q
\end{array}\right) \sum_{l=k}^{p-1}\left(\begin{array}{c}
p \\
p-1
\end{array}\right) \alpha_{q}^{2 p-2+2 t-2 j}\left(\begin{array}{c}
-\frac{1}{2} \\
l-k
\end{array}\right) \\
& +\left(\begin{array}{c}
-\frac{1}{2}-j \\
k-j
\end{array}\right) \sum_{t=0}^{j-1} K_{2 j+1, t} \sum_{q=0}^{p-1}(-1)^{q} \frac{1}{(2 p-2) !}\left(\begin{array}{c}
2 p-2 \\
q
\end{array}\right) \sum_{l=k}^{p-2} \sum_{s=0}^{p-2-l} c_{s} \alpha_{q}^{2 s+2 l+2 t-2 j}\left(\begin{array}{c}
-\frac{1}{2} \\
l-k
\end{array}\right) \\
& =\left(\begin{array}{c}
-\frac{1}{2}-j \\
k-j
\end{array}\right) \sum_{q=0}^{p-1}(-1)^{q} \frac{1}{(2 j+1)(2 p-2) !}\left(\begin{array}{c}
2 p-2 \\
q
\end{array}\right) \alpha_{q}^{2 p-2} \sum_{l=k}^{p-1}\left(\begin{array}{c}
p \\
p-1-l
\end{array}\right)\left(\begin{array}{c}
-\frac{1}{2} \\
l-k
\end{array}\right) \\
& =\left(\begin{array}{c}
-\frac{1}{2}-j \\
k-j
\end{array}\right) \frac{1}{2(2 j+1)} \sum_{l=k}^{p-1}\left(\begin{array}{c}
p-1-l \\
p-1
\end{array}\right)\left(\begin{array}{c}
1 \\
l-k
\end{array}\right) \\
& =\left(\begin{array}{c}
-\frac{1}{2}-j \\
k-j
\end{array}\right) \frac{1}{2(2 j+1)} \frac{(2 p-1) ! !}{(p-1-k) !(2 k+1) ! ! 2^{p-k-1}} \\
& =\frac{(-1)^{k-j}}{(k-j) !} \frac{2^{j}}{2^{k}} \frac{(2 k-1) ! !}{(2 j-1) ! !} \frac{1}{2(2 j+1)} \frac{(2 p-1) ! !}{(p-1-k) !(2 k+1) ! ! 2^{p-k-1}} \\
& =\frac{(-1)^{k-j}}{(k-j) !} \frac{2^{j}}{2^{2 p-1}(p-1) !} \frac{1}{(2 j+1) ! !} \frac{(2 p-1) !}{(p-1-k) !(2 k+1)}=N_{j}(p, k) .
\end{aligned}
$$

Agora estamos prontos para provar a conjectura 1.

Teorema 21. A Torção Analítica do cone $C_{\alpha} S_{l \operatorname{sen} \alpha}^{2 p-1}$ de ângulo $\alpha$ e comprimento l $>0$, sobre a esfera de dimensão ímpar $S^{2 p-1}$, com a métrica induzida pela imersão em $\mathbb{R}^{m+1}$, e condiçôes de contorno absoluta (onde $p>0$ ) é:

$$
\begin{aligned}
& \log T\left(C_{\alpha} S_{l \operatorname{sen} \alpha}^{2 p-1}\right)=\frac{1}{2} \log \operatorname{Vol}\left(C_{\alpha} S_{l \operatorname{sen} \alpha}^{2 p-1}\right) \\
& +\frac{(2 p-1) !}{4^{p}(p-1) !} \sum_{k=0}^{p-1} \frac{1}{(p-1-k) !(2 k+1) \nu^{2 k+1}} \sum_{j=0}^{k} \frac{(-1)^{k-j} 2^{j+1}}{(k-j) !(2 j+1) ! !}
\end{aligned}
$$

Demonstração. A prova segue usando resultados dos lemas 28 e 29 e da proposição 16 no teorema 19.

Observação 2. Temos duas notações para o cone sobre $S^{n}$. Quando denotamos $C_{l} S_{\operatorname{sen} \alpha}^{n}$ estamos nos referindo ao cone métrico limitado e então passamos a detalhar a seção. Quando denotamos $C_{\alpha} S_{l \operatorname{sen} \alpha}^{n}$ é o mesmo que $C_{l} S_{\mathrm{sen} \alpha}^{n}$, mas como neste caso podemos parametrizá-lo sem maiores problemas, estamos destacando a base do cone e, por isso, esta notação fica mais coerente. 
Corolário 8. A Torção Analítica de um cone sobre uma esfera de dimensão ímpar é

$$
\log T\left(C_{\alpha} S_{l \operatorname{sen} \alpha}^{2 p-1}\right)=\log \tau\left(C_{\alpha} S_{l \operatorname{sen} \alpha}^{2 p-1}\right)+A\left(S_{l \operatorname{sen} \alpha}^{2 p-1}\right),
$$

o que significa que não existe contribuição da singularidade e, além disso, pode ser escrita apenas com termos geométricos, ou seja,

$$
\log T\left(C_{\alpha} S_{l \operatorname{sen} \alpha}^{2 p-1}\right)=\frac{1}{2} \log \operatorname{Vol}\left(C_{\alpha} S_{l \operatorname{sen} \alpha}^{2 p-1}\right)+\frac{1}{2} \int_{S_{l \operatorname{sen} \alpha}^{2 p-1}} B\left(\nabla_{1}^{T C_{\alpha} S_{l \operatorname{sen} \alpha}^{2 p-1}}\right) .
$$




\section{Referências Bibliográficas}

[1] N. Berline, E. Getzler, M. Verge, Heat Kernels and Dirac Operators, 1991.

[2] M. Bordag, B. Geyer, K. Kirsten, E. Elizalde Zeta function determinant of the Laplace Operator on the D-dimensional ball, Comm. Math. Phys. 179, no. 1, (1996), 215-234.

[3] J.M. Bismut, W. Zhang, An extension of a theorem by Cheeger and Müller, Astérisque 205, (1992).

[4] J. Brüning, X. Ma, An anomaly formula for Ray-Singer metrics on manifolds with boundary, GAFA 16 (2006), 767-873.

[5] J. Brüning, R. Seeley, The resolvent expansion for second order regular singular operators, J. of Funct. An. 73 (1988), 369-415.

[6] J. Cheeger, Analytic torsion and the heat equation, Ann. Math. 109 (1979), 259-322.

[7] J. Cheeger, Hodge theory of complex cones, Proc. Luminy Conf., "Analyse et Topologie sur les Espaces singuliers", Asterisque 101-102, (1983), 118-134.

[8] J. Cheeger, On the spectral geometry of spaces with conical singularities, Proc. Nat. Acad. Sci. 76 (1979), 2103-2106.

[9] J. Cheeger, Spectral geometry of singular Riemannian spaces, J. Diff. Geom. 18 (1983), 575-657.

[10] J. Cheeger, On the Hodge theory of Riemannian pseudomanifolds, Proc. Sympos. Pure Math. 36 (1980), 91-146.

[11] M.M. Cohen, A course in Simple-Homotopy Theory, Graduate Text in Mathematics 10, 1973.

[12] E.T. Copson, An introduction to the theory of functions of a complex variables, Oxford University Press, 1950.

[13] X. Dai, H. Fang, Analytic torsion and R-torsion for manifolds with boundary, Asian J. Math. 4 (2000), 695-714. 
[14] T. de Melo, L. Hartmann, M. Spreafico, Reidemeister torsion and analytic torsion of discs, Boll. Unione Mat. Ital. 9 (2009), 529-533.

[15] T. de Melo, M. Spreafico, Reidemeister torsion and analytic torsion of spheres, J. Homotopy Relat. Struct., v. 4 (2009), 181-185.

[16] G. de Rham, Complexes à automorphismes et homéomorphie différentiables, Ann. Inst. Fourier, Grenoble 2 (1950), 51-67.

[17] M. P. do Carmo, Geometria Riemanniana, IMPA, 2005.

[18] J.S. Dowker, K. Kirsten, Spinors and forms on generalised cones, Comm. Anal. Geom. 7 (1999), no. 3, 641-679.

[19] W. Franz, Über die Torsion einer Überdeckung, J. Reine Angew. Math. 173 (1935), 245254.

[20] P. B. Gilkey, Invariance theory, the heat equation, and the Atiyah-Singer index theorem, Studies in Advanced Mathematics, 1995.

[21] I. S. Gradshteyn, I. M. Ryzhik, Table of integrals, Series and Products, Academic Press, 2007.

[22] L. Graham, D. R. Knuth, O. Patashnik, Concrete Mathetamics: a fundation fot computer science, Addison Wesley, Reading, MA, 1994.

[23] A. Ikeda, Y. Taniguchi, Spectra and eigenforms of the Laplacian on $S^{n}$ and $P^{n}(\mathbb{C})$, Osaka J. Math. 15 (1978), 515-546.

[24] L. Hartmann, M. Spreafico, Analytic torsion of cones over spheres, J. Math. Pures Appl, artigo aceito para publicação (2009).

[25] V. A. Krachmar, Problem book in algebra, 1974.

[26] J. Lott, M. Rothenberg, Analytic torsion for group actions, J. Differential Geom. 34 (1991), no. 2, 431-481.

[27] W. Lück, Analytic and topological torsion for manifolds with boundary and symmetry, J. Differential Geom. 37 (1993), 263-322.

[28] W. Lück, T. Schick, $L^{2}$-torsion of hyperbolic manifolds of finite volume, GAFA 9 (1999), 518-567.

[29] J. Milnor, A duality theorem for Reidemeister torsion, Ann. of Math. 76 (1962), 137-147.

[30] J. Milnor, Whitehead torsion, Bull. AMS 72 (1966), 358-426. 
[31] S. Morita, Geometry of Differential Forms, Translations of Mathematical Monographs. AMS, v. 201, 2001.

[32] W. Müller, Analytic torsion and R-torsion of Riemannian manifolds, Adv. Math. 28 (1978) 233-305.

[33] M. Nakahara, Geometry, Topology and Physics, Graduate student series in physics. Hadam Hilger 2003.

[34] M. Nagase, De Rham-Hodge theory on a manifold with cone-like singularities, Kodai Math. J., 1 (1982), 38-64.

[35] F. W. J. Olver, Asymptotics and special functions, AKP, 1997.

[36] D. B. Ray, I.M. Singer, R-torsion and the Laplacian on Riemannian manifolds, Adv. Math. 7 (1971), 145-210.

[37] D. B. Ray, Reidemeister torsion and the Laplacian on lens spaces, Adv. Math. 4 (1970), 109-126.

[38] K. Reidemeister, Homotopieringe und Linseräume, Hamburger Abhandl. 11 (1935), 102109.

[39] F. Rellich, Die zulässigen Randbedingungen bei den singulären Eigenwert-problem der mathematischen Physik, Math. Z. 49 (1943/44), 702-723.

[40] M. Spreafico, Zeta function and regularized determinant on projective spaces, Rocky Mount. Jour. Math. 33 (2003), 1499-1512.

[41] M. Spreafico, On the Bessel zeta function, Mathematika 51 (2004), 123-130.

[42] M. Spreafico, Zeta function and regularized determinant on a disc and on a cone, J. Geo. Phys. 54 (2005), 355-371.

[43] M. Spreafico, Zeta invariants for sequences of spectral type, special functions and the Lerch formula, Proc. Roy. Soc. Edinburgh 136A (2006), 863-887.

[44] M. Spreafico, Zeta invariants for Dirichlet series, Pacific. J. Math. 224 (2006), 180-199.

[45] M. Spreafico, The determinant for the Laplacian on forms and a torsion type invariant on a cone over the circle, Far East J. Math. Sc. 29 (2008), 353-368.

[46] M. Spreafico, Zeta invariants for double sequences of spectral type, preprint (2006), arXiv:math/0607816.

[47] B. Vertman, The Analytic Torsion of Manifolds with Boundary and Conical Singularities, PhD Thesis, Bonn University 2008. 
[48] G. N. Watson, A treatise on the theory of Bessel functions, Cambridge University Press, 1922.

[49] E. T. Whittaker, G. N. Watson, Modern Analysis, Cambridge University Press, 1940.

[50] L. Weng, Y. You, Analytic torsions of spheres, Int. J. Math. 7 (1996) 109-125.

[51] J. H. C. Whitehead, Simple homotopy types, Amer. J. Math. 72 (1950) 1-57. 


\section{Índice Remissivo}

$\alpha_{q}, 16$

* de Hodge, 12

$\mu_{q, n}, 16$

adjunto da derivada exterior $d^{\dagger}, 13$

classe de Euler, 11

complexo de de Rham, 8

condições de contorno

absoluta, 26

relativa, 26

cone

métrico, 14

métrico limitado, 14]

constante

de Cartan, 10

de Euler, 17

decomposição de Hodge

variedade com bordo, 27

variedade fechada, 13

derivada exterior $d, 7$

dualidade de Poincaré

para a Torção Analítica, 30

duplo fatorial, 52

elemento volume, 12

equação geodésica, 11

espectro positivo do Laplaciano do cone, 103

fibrado associado a uma representação, 25

forma

coexata, 13

cofechada, 13 conexão, 11

curvatura, 11

de volume, 12

diferencial, 6]

exata, 8

fechada, 8

harmônica, 13

integral de Berezin, 35

métrica

com estrutura produto próximo ao bordo, 34

Riemanniana, 9

operador Laplaciano, 13

Pfaffiano, 11

produto

exterior, 7

interno de formas, 13

tensorial, 6

wedge, 6

$r$-forma, 6

seqüência

espectralmente decomposta, 19

totalmente regular do tipo espectral com ordem infinita, 18

soma de Einstein, 5

tensor, 5

Teorema

comparação entre torções analíticas, 36 
de Cheeger Müller para variedades com bordo, 36

de Cheeger Müller para variedades com o bordo totalmente geodésico, 38

de Cheeger Müller para variedades que a métrica tem estrutura produto próxima ao bordo, 34

de Cheeger Müller, 33

Gauss-Bonet, 11

termo anômalo do bordo, 34

Torção

Analítica, 29

Analítica do cone sobre a $S^{1}, 69$

Analítica do cone sobre a $S^{2}, 73$

Analítica do cone sobre a $S^{3}, 84$

Analítica do cone sobre uma esfera de dimensão ímpar, 143

Analítica do cone sobre uma variedade fechada de dimensão ímpar, 131

Analítica do disco, 56

de Reidemeister, 23

de Reidemeister de um disco de dimensão $m$ no caso absoluto, 50

de Reidemeister de um disco de dimensão $m$ no caso relativo, 49

variedade

com bordo totalmente geodésico, 37

com singularidade do tipo cônico, 14

Riemanniana, 9 